\title{
Algebraic, Analytic, and Combinatorial Properties of Power Product Expansions in Two Independent Variables.
}

\author{
Mohamed Ammar Elewoday \\ maelewoday@mix.wvu.edu
}

Follow this and additional works at: https://researchrepository.wvu.edu/etd

Part of the Analysis Commons, Discrete Mathematics and Combinatorics Commons, and the Harmonic Analysis and Representation Commons

\section{Recommended Citation}

Elewoday, Mohamed Ammar, "Algebraic, Analytic, and Combinatorial Properties of Power Product Expansions in Two Independent Variables." (2021). Graduate Theses, Dissertations, and Problem Reports. 8308.

https://researchrepository.wvu.edu/etd/8308

This Dissertation is protected by copyright and/or related rights. It has been brought to you by the The Research Repository @ WVU with permission from the rights-holder(s). You are free to use this Dissertation in any way that is permitted by the copyright and related rights legislation that applies to your use. For other uses you must obtain permission from the rights-holder(s) directly, unless additional rights are indicated by a Creative Commons license in the record and/ or on the work itself. This Dissertation has been accepted for inclusion in WVU Graduate Theses, Dissertations, and Problem Reports collection by an authorized administrator of The Research Repository @ WVU.

For more information, please contact researchrepository@mail.wvu.edu. 
Algebraic, Analytic, and Combinatorial Properties of Power Product Expansions in Two Independent Variables.

Mohamed Elewoday

Dissertation submitted to the

Eberly College of Arts and Sciences

at West Virginia University

in partial fulfillment of the requirements

for the degree of

Doctor of Philosophy

in

Mathematics

Harry Gingold, Ph.D, Chair

Jocelyn Quaintance, Ph.D

Harumi Hattori, Ph.D

Rong Luo, Ph.D

Jerzy Wojciechowski, Ph.D

Department of Mathematics

Morgantown, WV

2021

Keywords: infinite products, Matrix functions, power series in two independent variables, non-commutative, convergence, partitions .

Copyright 2021 Mohamed Elewoday 


\section{ABSTRACT \\ Algebraic, Analytic, and Combinatorial Properties of Power Product Expansions in Two Independent Variables.}

\section{Mohamed Elewoday}

Let $F(x, y)=I+\sum_{\substack{p=1 \\ m+n=p}}^{\infty} A_{m, n} x^{m} y^{n}$ be a formal power series, where the coefficients $A_{m, n}$ are either all matrices or all scalars. We expand $F(x, y)$ into the formal products $\prod_{\substack{p=1 \\ m+n=p}}^{\infty}\left(I+G_{m, n} x^{m} y^{n}\right), \quad \prod_{\substack{p=1 \\ m+n=p}}^{\infty}\left(I-H_{m, n} x^{m} y^{n}\right)^{-1}$, namely the power product expansion in two independent variables and inverse power product expansion in two independent variables respectively. By developing new machinery involving the majorizing infinite product, we provide estimates on the domain of absolute convergence of the infinite product via the Taylor series coefficients of $F(x, y)$. This machinery introduces a myriad of "mixed expansions", uncovers various algebraic connections between the $\left(A_{m, n}\right)$ and the $\left(G_{m, n}\right)$, and uncovers various algebraic connections between the $\left(A_{m, n}\right)$ and the $\left(H_{m, n}\right)$, and leads to the identification of the domain of absolute convergence of the power product and the inverse power product as a Cartesian product of polydiscs. This makes it possible to use the truncated power product expansions $\prod_{\substack{p=1 \\ m+n=p}}^{P}\left(1+G_{m, n} x^{m} y^{n}\right), \prod_{\substack{p=1 \\ m+n=p}}^{P}\left(1-H_{m, n} x^{m} y^{n}\right)^{-1}$ as approximations to the analytic function $F(x, y)$. The results are made possible by certain algebraic properties characteristic of the expansions. Moreover, in the case where the coefficients $A_{m, n}$ are scalars, we derive two asymptotic formulas for the $G_{m, n}, H_{m, n}$, with $m$ fixed, associated with the majorizing power series. We also discuss various combinatorial interpretations provided by these power product expansions. 


\section{Acknowledgements}

First and foremost, I am most indebted to my supervisors, Prof. Harry Gingold and Prof. Jocelyn Quaintance, for their continued encouragement and support over these last few years. It is a pleasure to work under their supervision. Without Allah and then them, this work could not have come about. I would also like to thank my other committee members: Prof. Harumi Hattori, Prof. Jerzy Wojciechowski, and Prof. Rong Luo, for their help during my studies. And finally, I would like to thank the Department of Mathematics and Eberly College of Arts and Sciences at West Virginia University for providing me with an excellent study environment and support during my study as a graduate student. 


\section{Dedication}

To

the memory of my parents,

my wife $\underline{\text { Afaf, }}$

and

my lovely children Aisha, Loai, Roaa, Laith, and Tamim 


\section{Contents}

1 Factorization of Bivariate Analytic functions Via Power Product Expansion 1

1.1 Introduction . . . . . . . . . . . . . . . . 1

1.2 Two Algebraic Formulas for the Coefficients of a Power Product Expansion . . . . . . . . . . . . . . . 4

1.3 Structure Property of the Coefficients of a Power Product Expansion 9

1.4 Convergence Criteria for Power Product Expansion . . . . . . . 15

1.5 Asymptotic Approximation for a Special Power Product Expan-

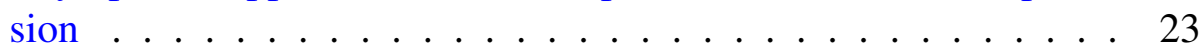

1.6 A combinatorial interpretation for PPE2 . . . . . . . . 30

2 Factorization of Bivariate Taylor Series via Inverse Power Products 37

2.1 Introduction . . . . . . . . . . . . . . . . . . . . 37

2.2 Two Algebraic Formulas for the Coefficients of Inverse Power Product Expansion . . . . . . . . . . . . . . . . 40

2.3 Structure Property of the Coefficients of an Inverse Power Product Expansion . . . . . . . . . . . . . . . . . . . 43

2.4 Convergence Criteria For Inverse Power Product . . . . . . . . 48

2.5 Asymptotic Approximation for the Majorizing Inverse Power Product Expansion . . . . . . . . . . . . . . . . . . . 54

2.6 Combinatorial Interpretations for IPPE2 $\ldots \ldots \ldots$

3 Factorization of Bivariate Matrix Power Series Via Power Product Expansion $\quad 69$

3.1 Introduction . . . . . . . . . . . . . . . . . . . . . . 69

3.2 Matrix Power Product Expansions . . . . . . . . . . . . 71

3.3 Algebraic Formula for the Coefficients of a Matrix Power Product Expansion . . . . . . . . . . . . . . . . . 74 
3.4 Structure Property of the Coefficients of a Matrix Power Product Expansion . . . . . . . . . . . . . . . . 74

3.5 Convergence Criteria for MPPE2's . . . . . . . . . . . . . . . . . 79

3.6 Matrix MPPE2's Induced by Scalar Functions . . . . . . . . . . . . 90

3.7 Illustrative Examples . . . . . . . . . . . . . . . 99

4 Factorization of Bivariate Matrix Power Series via Power Inverse Prod$\begin{array}{lr}\text { uct Expansion } & 114\end{array}$

4.1 Introduction . . . . . . . . . . . . . . . . . . 114

4.2 Algebraic Formulas for the Coefficients of Inverse Matrix Power

Product Expansion . . . . . . . . . . . . . . 116

4.3 Structure Property of the Coefficients of an Inverse Power Product

Expansion ........................ 116

4.4 Convergence criteria for IMPPE2's . . . . . . . . . . . . . . . . . . . . . . . . . . . . . . 135

4.5 Matrix IMPPE2's induced by scalar functions . . . . . . . . . 135

4.6 Illustrative examples ................... 151

$\begin{array}{lrr}\text { A Double Series } & 174\end{array}$

A.1 Double sequence . . . . . . . . . . . . . . 174

A.2 Monotone Double Sequences . . . . . . . . . . . . . . . 179

A.3 Convergence of Double Series . . . . . . . . . . . . . . 181

A.4 Cauchy Product of Double Series . . . . . . . . . . . . . . 188

A.5 Double Series with Non-negative Terms . . . . . . . . . . . . . 189

A.6 Absolute Convergence of Double Series . . . . . . . . . . . . . 194

A.7 Convergence Tests for Double Series . . . . . . . . . . . . . 197

A.8 Double Power Series . . . . . . . . . . . . . . . . . 203

A.9 Taylor Double Series and Taylor Series . . . . . . . . . . . 210

$\begin{array}{lr}\text { Bibliography } & 215\end{array}$ 


\section{Chapter 1}

\section{Factorization of Bivariate Analytic functions Via Power Product Expansion}

\subsection{Introduction}

The subject of this chapter is the expansion of $f(x, y)=1+\sum_{\substack{p=1 \\ m+n=p}}^{\infty} a_{m, n} x^{m} y^{n}$ with complex coefficients into an infinite product, where either the defining expression for $f(x, y)$ is treated as a formal power series expansion or $f(x, y)$ is an analytic function with $f(0,0)=1$. The right side of

$$
f(x, y)=\prod_{\substack{q=1 \\ m+n=q}}^{\infty}\left(1+g_{m, n} x^{m} y^{n}\right)
$$

is defined to be power product expansion in two independent variables, (or PPE2), and provides a factorization of $f(x, y)$. Observe that finite truncations of the PPE2, namely $\left\{\prod_{\substack{q=1 \\ m+n=q}}^{P}\left(1+g_{m, n} x^{m} y^{n}\right)\right\}_{P=1}^{\infty}$, provide polynomial approximations for $f(x, y)$.

A few special cases of PPE2s appear throughout the literature. The infinite product with elementary factors $\left(1+x^{m} y^{n}\right)$ is used as a generating function to 
determine the sequence $\left(p_{d}(m, n)\right)_{m, n=0}^{\infty}$

$$
\sum_{\substack{p=1 \\ m+n=p}}^{\infty} p_{d}(m, n) x^{m} y^{n}=\prod_{\substack{q=1 \\ m+n=q}}^{\infty}\left(1+x^{m} y^{n}\right)
$$

where $p_{d}(m, n)$ is the number of partitions of $(m, n) \in \mathbb{N}_{0} \times \mathbb{N}_{0}$ into distinct parts. There is also Euler's infinite product in two variables, namely

$$
\sum_{\substack{p=1 \\ m+n=p}}^{\infty} p(m, n) x^{m} y^{n}=\prod_{\substack{q=1 \\ m+n=q}}^{\infty}\left(1-x^{m} y^{n}\right)^{-1}
$$

where $p(m, n)$ represents the number of partitions of $(m, n) \in \mathbb{N}_{0} \times \mathbb{N}_{0}$ into unrestricted parts [1], [10]. As these two classical examples suggest, the expansion of a general infinite product $\prod_{\substack{q=1 \\ m+n=q}}^{\infty}\left(1+g_{m, n} x^{m} y^{n}\right)$ into a power series $1+\sum_{\substack{p=1 \\ m+n=p}}^{\infty} a_{m, n} x^{m} y^{n}$ generates an infinite sequence of coefficients $a_{m, n}$ that count the number of arrangements in a variety of combinatorial configurations. The convergence properties of $\prod_{\substack{q=1 \\ m+n=q}}^{\infty}\left(1+g_{m, n} x^{m} y^{n}\right)$ and its companion power series are very important for similar reasons since they are crucial in determining the order of growth of the coefficients $a_{m, n}=p_{d}(m, n)$, and $a_{m, n}=p(m, n)$ as $m+n$ goes to infinity.

Much research has been done on one variable power product expansions; see [8],[9],[29],[31],[32]. However, only sporadic work has been done for power product expansions in two independent variables. Cheema [10] has combinatorial results involving PPE2s and vector partition identities, while Feld and Newman [13] have estimated of the domain of convergence of the PPE2 by expressing the convergence of infinite product in terms of the growth of the coefficients of the Taylor series of $\log f(x, y)=\sum_{\substack{p=1 \\ m+n=p}}^{\infty} c_{m, n} x^{m} y^{n}$. There are advantages and insights gained by the logarithm of the function $f(x, y)$. However, they come with a penalty since they provide an indirect expression for the coefficients $g_{m, n}$ in terms of the coefficients $c_{1,0}, c_{0,1}, \ldots, c_{i, j}$ rather than a direct expression of $g_{m, n}$ in terms of the $a_{1,0}, a_{0,1}, \ldots, a_{i, j}$. Consequently, an estimate for the domain of convergence of the PPE2 is not directly expressed in terms of the order of growth of the coefficients $a_{1,0}, a_{0,1}, \ldots, a_{i, j}$. This shortcoming was remedied in the case of one independent 
variable by H. Gingold, A. Knopfmacher, and J. Quaintance who expressed $g_{n}$ as a polynomial in the variables $\left(a_{i}\right)_{i=1}^{n}$ [17], [19], [22]. In what is perhaps the most important result of this chapter, we extend this polynomial based approach of Gingold et al. to the case of two independent variables and thus provide an estimate for the domain of convergence of a PPE2 directly in terms of the power series coefficients $\left(a_{m, n}\right)_{\substack{m, n=0 \\ m+n=1}}^{\infty}$.

This chapter contains two major results. The first main result, Theorem 1.4.1, provides a domain of convergence for $f(x, y)=\prod_{\substack{q=1 \\ m+n=q}}^{\infty}\left(1+g_{m, n} x^{m} y^{n}\right)$, in terms of the "majorizing infinite product"

$$
1-\sum_{\substack{p=1 \\ m+n=p}}^{\infty} s^{m+n} x^{m} y^{n}=\sum_{\substack{p=1 \\ m+n=p}}^{\infty}\left(1-E_{m, n} x^{m} y^{n}\right)
$$

where $s: \equiv \sup _{\substack{p=1 \\ m+n=p}}\left|a_{m, n}\right|^{\frac{1}{m+n}}$. The method of majorizing series is well established in the analytic theory of complex variables; see J.B. Conway [5] and S. Ghorpade and B. Limaye [28]. However, the same did not hold true for product expansions until the publication of [22]. The proof of Theorem 1.4.2 makes use of the fact that $\left|g_{m, n}\right| \leq E_{m, n}$. See Theorem 1.4.1. The proof of this inequality involves generalizing the recursive algebraic procedure initiated in [23] and [22] to deduce that if $a_{m, n} \leq 0$, then $g_{m, n} \leq 0$. See Theorem 1.3.2 and Equation (1.28). In the case of Theorem 1.4.2, the majorizing power

$$
1-\sum_{\substack{p=1 \\ m+n=p}}^{\infty} s^{m+n} x^{m} y^{n}=\frac{1-2 s(x+y)+2 s^{2} x y}{(1-s x)(1-s y)} .
$$

By setting $y=0$, Equation (1.4) provides the majorizing function for one variable case, namely $\frac{1-2 s x}{1-s x}$. In [19] Gingold and Knopfmacher showed that asymptotic value of $\log \frac{1-2 s x}{1-s x}=\sum_{n=0}^{\infty} d_{n} x^{n}$ coincides with the asymptotic value of its power product expansion $\prod_{n=1}^{\infty}\left(1+g_{n} x^{n}\right)$, i.e. $\lim _{n \rightarrow \infty} \frac{g_{n}}{d_{n}}=1$. The second major outcome of this chapter, Theorem 5.1, extends this aforementioned asymptotic result to PPE2s, where for fixed $M$, we show that $\lim _{n \rightarrow \infty} \frac{g_{M, n}}{d_{M, n}}=1$ whenever $\log \frac{1-2 s(x+y)+2 s^{2} x y}{(1-s x)(1-s y)}=\sum_{\substack{p=1 \\ m+n=p}}^{\infty} d_{m, n} x^{m} y^{n}$. This chapter is organized as follows. In 
Section 2, we study the expansion of a power series into a PPE2 and provide two algebraic representations for the coefficients $g_{m, n}$ as a multivariate polynomials in $\left(a_{m, n}\right)_{\substack{m, n=0 \\ m+n=1}}^{\infty}$. In Section 3, we provide another way to express recursively the coefficients $g_{m, n}$ as a multivariate polynomial of the variables $a_{m, n}$. The algebraic result of Section 3 reveals an intriguing property of these expansions. If $a_{m, n} \leq 0$, then the coefficients $g_{m, n}$ in the PPE2 are non-positive. In Section 4, we exploit the non-positivity result of Section 3 to determine convergence conditions of the PPE2 in terms of a majorizing power product. In Section 5, we provide an asymptotic formula for the $g_{m, n}$ associated with $1-\sum_{\substack{p=1 \\ m+n=p}}^{\infty} s^{m+n} x^{m} y^{n}$, where $s: \equiv \sup _{\substack{p=1 \\ m+n=p}}\left|a_{m, n}\right|^{\frac{1}{m+n}}$, while in Section 6 we provide combinatorial interpretations for PPE2 in term of partitions of $(m, n)$. Section 6 also provides a convergence theorem for the PPE2s associated with combinatorial sequences which states that the PPE2 and its Taylor series have the same domain of convergence; see Corollary 1.6.1.

\subsection{Two Algebraic Formulas for the Coefficients of a Power Product Expansion}

In this section and the next section we study the expansion of a two variable power series into a PPE2 and provide three algebraic representations for the coefficients $g_{m, n}$ as polynomials of the $\left(a_{m, n}\right)_{m, n=0}^{\infty}$. Unlike the convergence of the serie $\sum_{n=0}^{\infty} a_{n}$, the convergence of the double series $\sum_{m, n}^{\infty} b_{m, n}$ requires additional considerations. In order to justify the particular order of summation that we utilize throughout this article, we briefly recall some theoretical results. Following J. Morrow [38], we define $\sum_{m, n} b_{m, n}$ as a double $m n$ indexed infinite series of complex numbers. (In our particular case, $b_{m, n}=a_{m, n} x^{m} y^{n}$.) We define the associated sequence of partial sums $\left(s_{m, n}\right)$ via the finite sum

$$
s_{m, n}=\sum_{j=1}^{m} \sum_{k=1}^{n} b_{j, k}
$$

We say $\sum_{m, n} b_{m, n}$ converges if and only if $\lim _{m, n \rightarrow \infty} s_{m, n}$ converges. We say $\sum_{m, n} b_{m, n}$ converges absolutely if and only if $\sum_{m, n}\left|b_{m, n}\right|$. The crucial result, [[38], Theorem 
2], states that if $\sum_{m, n} b_{m, n}$ absolutely converges, then $\sum_{m, n} b_{m, n}$ converges and that the sum of $\sum_{m, n} b_{m, n}$ can be computed by any rearrangement of terms. Since we will be working with either doubly indexed formal power series or doubly indexed absolutely convergent series, without loss of generality we define

$$
\begin{aligned}
\sum_{m, n} b_{m, n} & :=b_{0,0}+\sum_{\substack{p=1 \\
m+n=p}}^{\infty} b_{m, n} \\
& =b_{0,0}+b_{1,0}+b_{0,1}+b_{2,0}+b_{1,1}+b_{0,2}+\ldots .
\end{aligned}
$$

See Definitions 1.2.1 and 1.2.2. But before we even define what we mean by a PPE2, we need some preliminary notation and definitions.

Remark 1.2.1. Throughout this work, $\mathbb{N}_{0}:=\mathbb{N} \cup\{0\}$.

Definition 1.2.1. We define a well ordering $\prec$ on $\mathbb{N}_{0} \times \mathbb{N}_{0}$ via the following binary relation: for $\left(n_{1}, n_{2}\right),\left(n_{3}, n_{4}\right) \in \mathbb{N}_{0} \times \mathbb{N}_{0}$, we have $\left(n_{1}, n_{2}\right) \prec\left(n_{3}, n_{4}\right)$ if either

1. $n_{1}+n_{2}<n_{3}+n_{4}$ or

2. $n_{1}+n_{2}=n_{3}+n_{4}$ and $n_{1}>n_{3}$, or equivalently $n_{2}<n_{4}$.

Here $<$ is the usual order relation on $\mathbb{N}_{0}$. See Figure 1. 


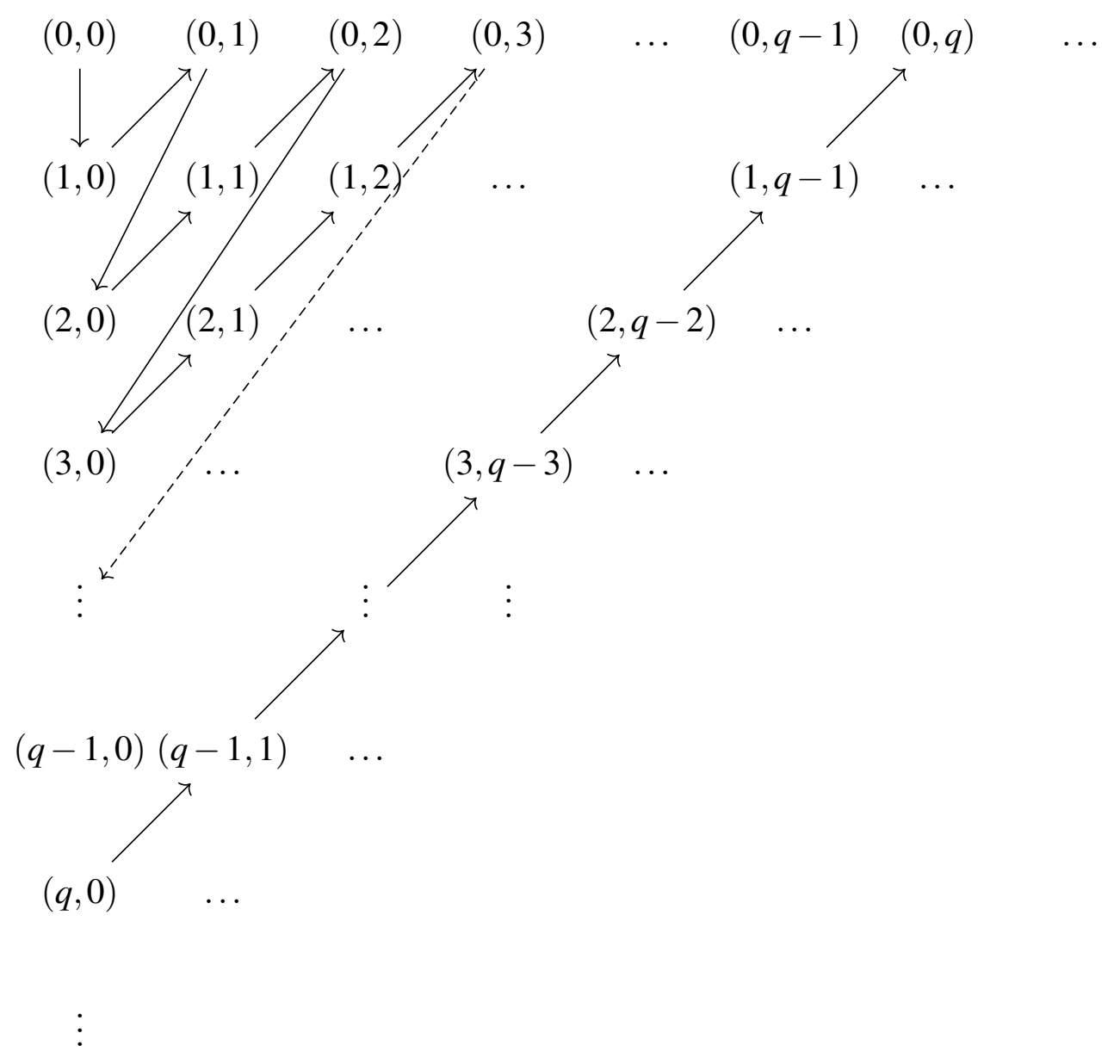

Figure 1.1: Ordering of $(m, n) \in \mathbb{N}_{0} \times \mathbb{N}_{0}$ according to Definition 1.2.1.

For example, $(1,0) \prec(2,0) \prec(1,1) \prec(0,2)$.

Definition 1.2.2. Given a formal power series $f(x, y)=1+\sum_{\substack{p=1 \\ m+n=p}}^{\infty} a_{m, n} x^{m} y^{n}$ or analytic function $f(x, y)$ with $f(0,0)=1$ whose Taylor series representation is defined by the well-ordering of Definition 1.2.1, namely

$$
f(x, y)=1+\sum_{\substack{p=1 \\ m+n=p}}^{\infty} a_{m, n} x^{m} y^{n}=1+a_{1,0} x+a_{0,1} y+a_{2,0} x^{2}+\ldots
$$


we define the power product expansion of $f(x, y)$ in two independent variables, denoted by PPE2, as

$$
\begin{aligned}
f(x, y)= & \prod_{\substack{q=1 \\
m+n=q}}^{\infty}\left(1+g_{m, n} x^{m} y^{n}\right)=\left(1+g_{1,0} x^{1} y^{0}\right)\left(1+g_{0,1} x^{0} y^{1}\right)\left(1+g_{2,0} x^{2} y^{0}\right) . \\
& \left(1+g_{1,1} x^{1} y^{1}\right)\left(1+g_{0,2} x^{0} y^{2}\right)\left(1+g_{3,0} x^{3} y^{0}\right)\left(1+g_{2,1} x^{2} y^{1}\right) \ldots,
\end{aligned}
$$

where the right equality of (1.7) follows from the conventions of Definition 1.2.1.

Definition 1.2.2 provides a first means of obtaining an algebraic representation of $g_{m, n}$ in terms of $\left(a_{m, n}\right)$. But we will need one more definition to describe this algebraic result.

Definition 1.2.3. Let $(m, n) \in \mathbb{N}_{0} \times \mathbb{N}_{0}$. A partition of $(m, n)$ is a collection $\left\{\left(p_{1}, p_{1}^{\prime}\right), \ldots,\left(p_{k}, p_{k}^{\prime}\right)\right\} \subseteq \mathbb{N}_{0} \times \mathbb{N}_{0}$ such that $\sum_{i=1}^{k}\left(p_{i}, p_{i}^{\prime}\right)=(m, n)$. The summands or parts $\left(p_{i}, p_{i}^{\prime}\right)$ need not be distinct, and the order of the summands is immaterial. Let $p((m, n))$ denote the number of partitions of $(m, n)$, and $p_{d}((m, n))$ denote the number of partitions of $(m, n)$ with distinct parts, where $p((0,0))=p_{d}((0,0))=$ 1.

For example, the partitions of $(2,2)$ are

$$
\begin{aligned}
& \quad\{(2,2),(2,0)+(0,2),(2,1)+(0,1),(1,2)+(1,0),(1,1)+(0,1)+(1,0)\} \cup \\
& \quad\{(2,0)+(0,1)+(0,1),(1,1)+(1,1),(1,0)+(0,1)+(1,0)+(0,1),(1,0)+(1,0)+(0,2)\}, \\
& \text { so } p((2,2))=9 \text {, and } p_{d}((2,2))=5 \text {. }
\end{aligned}
$$

Let

$$
f(x, y)=1+\sum_{\substack{p=1 \\ m+n=p}}^{\infty} a_{m, n} x^{m} y^{n}=\prod_{\substack{q=1 \\ m+n=q}}^{\infty}\left(1+g_{m, n} x^{m} y^{n}\right) .
$$

By expanding the PPE2 of (1.8) into a formal power series, coefficient comparison shows that

$$
a_{m, n}=\sum_{\substack{i_{1}+i_{2}+\ldots+i_{r}=m \\ j_{1}+j_{2}+\ldots+j_{r}=n \\(1,0) \preceq\left(i_{1}, j_{1}\right) \prec \cdots \prec\left(i_{r}, j_{r}\right) \preceq(m, n)}} g_{i_{1}, j_{1}} g_{i_{2}, j_{2}} \ldots g_{i_{r}, j_{r}},
$$

where the summation runs over all partitions of $(m, n)$ into distinct parts. 
Equation (1.9) can be rewritten as

$$
g_{m, n}=a_{m, n}-\left(\sum_{\substack{i_{1}+i_{2}+\ldots+i_{r}=m \\ j_{1}+j_{j}+\ldots+j_{r}=n,(1,0)\left(i_{1}, j_{1}\right)<\cdots<\left(i_{r}, j_{r}\right) \prec(m, n), r \geq 2}} g_{i_{1}, j_{1}} g_{i_{2}, j_{2} \ldots g_{i_{r}, j_{r}}}\right),
$$

To obtain the second algebraic formula which writes $g_{m, n}$ in terms of $\left(a_{m, n}\right)$, start with Equation (1.8) and take the logarithm of both sides to obtain

$$
\log (f(x, y))=\log \left(1+\sum_{\substack{p=1 \\ m+n=p}}^{\infty} a_{m, n} x^{m} y^{n}\right)=\sum_{\substack{p=1 \\ m+n=p}}^{\infty} d_{m, n} x^{m} y^{n},
$$

and to obtain

$$
\begin{aligned}
\log (f(x, y))=\log \prod_{\substack{q=1 \\
m+n=q}}^{\infty}\left(1+g_{m, n} x^{m} y^{n}\right) & :=\sum_{\substack{q=1 \\
m+n=q}}^{\infty} \log \left(1+g_{m, n} x^{m} y^{n}\right) \\
& :=\sum_{\substack{q=1 \\
m+n=q}}^{\infty} \sum_{l=1}^{\infty} \frac{(-1)^{l-1}\left(g_{m, n} x^{m} y^{n}\right)^{l}}{l} .
\end{aligned}
$$

Compare the coefficient of $x^{M} y^{N}$ on both sides of Equation (1.11) to obtain the formulas

$$
\begin{aligned}
& d_{M, N}=\frac{1}{M} \sum_{\substack{m \mid M \\
n=\frac{N m}{M}}}(-1)^{\frac{M}{m}-1} m\left(g_{m, n}\right)^{\frac{M}{m}}, \quad M \neq 0, \\
& d_{M, N}=\frac{1}{N} \sum_{\substack{n \mid N \\
m=\frac{M n}{N}}}(-1)^{\frac{N}{n}-1} n\left(g_{m, n}\right)^{\frac{N}{n}}, \quad N \neq 0 .
\end{aligned}
$$

Take Equation (1.12) and set $m=M$ to obtain

$$
g_{M, N}=d_{M, N}+\frac{1}{M} \sum_{\substack{m \mid M \\ m \neq M \\ n=\frac{N m}{M}}} m\left(-g_{m, \frac{N m}{M}}\right)^{\frac{M}{m}} .
$$


Similarly, take Equation (1.13) and set $n=N$ to obtain

$$
g_{M, N}=d_{M, N}+\frac{1}{N} \sum_{\substack{n \mid N \\ n \neq N \\ m=\frac{M n}{N}}} n\left(-g_{\frac{M n}{N}, n}\right)^{\frac{N}{n}}
$$

If $\operatorname{gcd}(M, N)=1$, then we readily deduce from Equations (1.14) and (1.15) that

$$
g_{M, N}=d_{M, N}
$$

\subsection{Structure Property of the Coefficients of a Power Product Expansion}

There is still another way to recursively express the coefficients $g_{m, n}$ as a multivariate polynomial in the variables $a_{m, n}$. Start by rewriting Equation (1.8) as

$$
f(x, y)=1+\sum_{\substack{p=1 \\ m+n=p}}^{\infty} A_{(1,0),(m, n)} x^{m} y^{n}=\prod_{\substack{q=1 \\ m+n=q}}^{\infty}\left(1+g_{m, n} x^{m} y^{n}\right)
$$

where $A_{(1,0),(m, n)}=a_{m, n}$ for all $(m, n) \in \mathbb{N}_{0} \times \mathbb{N}_{0}$. By using the well ordering of Definition 1.2.1 to factor $\prod_{\substack{q=1 \\ m+n=q}}^{\infty}\left(1+g_{m, n} x^{m} y^{n}\right)$, we get the following recursive system of equations: first,

$$
\begin{aligned}
f(x, y)=1+\sum_{\substack{p=1 \\
m+n=p}}^{\infty} A_{(1,0),(m, n)} x^{m} y^{n} & =\left(1+g_{1,0} x\right) \prod_{\substack{q=1 \\
m+n=q \\
(m, n) \succeq(0,1)}}^{\infty}\left(1+g_{m, n} x^{m} y^{n}\right) \\
& =\left(1+g_{1,0} x\right)\left[1+\sum_{\substack{p=1 \\
m+n=p \\
(m, n) \succeq(0,1)}}^{\infty} A_{(0,1),(m, n)^{2}} x^{m} y^{n}\right] ;
\end{aligned}
$$


secondly,

$$
\begin{aligned}
1+\sum_{\substack{p=1 \\
m+n=p \\
(m, n) \succeq(0,1)}}^{\infty} A_{(0,1),(m, n)} x^{m} y^{n} & =\left(1+g_{0,1} y\right) \prod_{\substack{q=2 \\
m+n=q}}^{\infty}\left(1+g_{m, n} x^{m} y^{n}\right) \\
& =\left(1+g_{0,1} y\right)\left[1+\sum_{\substack{p=2 \\
m+n=p}}^{\infty} A_{(2,0),(m, n)} x^{m} y^{n}\right]
\end{aligned}
$$

and thirdly,

$$
\begin{aligned}
1+\sum_{\substack{p=2 \\
m+n=p \\
(m, n) \succeq(2,0)}}^{\infty} A_{(2,0),(m, n)} x^{m} y^{n} & =\left(1+g_{2,0} x^{2}\right) \prod_{\substack{q=2 \\
m+n=q \\
(m, n) \succeq(1,1)}}^{\infty}\left(1+g_{m, n} x^{m} y^{n}\right) \\
& =\left(1+g_{2,0} x^{2}\right)\left[1+\sum_{\substack{p=2 \\
m+n=p \\
(m, n) \succeq(1,1)}}^{\infty} A_{(1,1),(m, n)} x^{m} y^{n}\right] .
\end{aligned}
$$

Continue this process inductively to define

$$
\begin{gathered}
1+\sum_{\substack{p=\ell \\
m+n=p \\
(m, n) \succeq\left(i_{k}, j_{k}\right)}}^{\infty} A_{\left(i_{k}, j_{k}\right),(m, n)} x^{m} y^{n}=\left(1+g_{i_{k}, j_{k}} x^{i_{k}} y^{j_{k}}\right) \prod_{\substack{q=\ell \\
m+n=q \\
(m, n) \succeq\left(i_{k+1}, j_{k+1}\right)}}^{\infty}\left(1+g_{m, n} x^{m} y^{n}\right) \\
=\left(1+g_{i_{k}, j_{k}} x^{i_{k}} y^{j_{k}}\right)\left[1+\sum_{\substack{p=\ell \\
m+n=p \\
(m, n) \succeq\left(i_{k+1}, j_{k+1}\right)}}^{\infty} A_{\left(i_{k+1}, j_{k+1}\right),(m, n)} x^{m} y^{n}\right] .
\end{gathered}
$$

We expand the right hand side of Equation (1.17) and compare the coefficient of $x^{M} y^{N}$ to obtain

$$
A_{\left(i_{k}, j_{k}\right),(M, N)}=A_{\left(i_{k+1}, j_{k+1}\right),(M, N)}+g_{i_{k}, j_{k}} A_{\left(i_{k+1}, j_{k+1}\right),\left(M-i_{k}, N-j_{k}\right)} .
$$

If $(M, N)=\left(i_{k}, j_{k}\right)$, Equation (1.18) reduces to

$$
A_{\left(i_{k}, j_{k}\right),\left(i_{k}, j_{k}\right)}=g_{i_{k}, j_{k}} .
$$

Thus Equation (1.18) becomes

$$
A_{\left(i_{k+1}, j_{k+1}\right),(M, N)}=A_{\left(i_{k}, j_{k}\right),(M, N)}-A_{\left(i_{k}, j_{k}\right),\left(i_{k}, j_{k}\right)} A_{\left(i_{k+1}, j_{k+1}\right),\left(M-i_{k}, N-j_{k}\right)} .
$$


Next, use Equation (1.19) and the binomial theorem to rewrite Equation (1.17) as

$$
\begin{aligned}
& 1+\sum_{\substack{p=\ell \\
m+n=p,(m, n) \succeq\left(i_{k+1}, j_{k+1}\right)}}^{\infty} A_{\left(i_{k+1}, j_{k+1}\right),(m, n)} x^{m} y^{n}=\left(1+g_{i_{k}, j_{k}} x^{i_{k}} y^{j_{k}}\right)^{-1}\left[1+\sum_{\substack{p=\ell \\
m+n=p \\
(m, n) \succeq\left(i_{k}, j_{k}\right)}}^{\infty} A_{\left(i_{k}, j_{k}\right),(m, n)} x^{m} y^{n}\right] \\
& =\left[1+\sum_{\alpha=1}^{\infty}(-1)^{\alpha}\left(g_{i_{k}, j_{k}} x^{i_{k}} y^{j_{k}}\right)^{\alpha}\right]\left[1+\sum_{\substack{p=\ell \\
m+n=p \\
(m, n) \succeq\left(i_{k}, j_{k}\right)}}^{\infty} A_{\left(i_{k}, j_{k}\right),(m, n)} x^{m} y^{n}\right] \\
& =\left[1+\sum_{\alpha=1}^{\infty}(-1)^{\alpha} A_{\left(i_{k}, j_{k}\right),\left(i_{k}, j_{k}\right)}^{\alpha}\left(x^{i_{k}} y^{j_{k}}\right)^{\alpha}\right]\left[1+\sum_{\substack{p=\ell \\
m+n=p \\
(m, n) \succeq\left(i_{k}, j_{k}\right)}}^{\infty} A_{\left(i_{k}, j_{k}\right),(m, n)} x^{m} y^{n}\right] .
\end{aligned}
$$

By equating the coefficient of $x^{s} y^{t}$ on both sides of Equation (1.21), we get

$$
A_{\left(i_{k+1}, j_{k+1}\right),(s, t)}=\sum_{\substack{\alpha i_{k}+m=s \\ \alpha j_{k}+n=t}}(-1)^{\alpha} A_{\left(i_{k}, j_{k}\right),\left(i_{k}, j_{k}\right)}^{\alpha} A_{\left(i_{k}, j_{k}\right),(m, n)} .
$$

Equation (1.22) will be the main tool for proving the following result.

Theorem 1.3.1. Let $\left(i_{k}, j_{k}\right) \in \mathbb{N}_{0} \times \mathbb{N}_{0} \backslash\{(0,0)\}$. Define $A_{\left(i_{k}, j_{k}\right),(0,0)}=1$ and $A_{\left(i_{k}, j_{k}\right),(m, n)}=0$ for $(1,0) \preceq(m, n) \preceq\left(i_{k-1}, j_{k-1}\right)$. Assume that $A_{\left(i_{k}, j_{k}\right),(m, n)} \leq 0$ for all $\left(i_{k}, j_{k}\right) \preceq(m, n)$. Then $A_{\left(i_{k+1}, j_{k+1}\right),(s, t)} \leq 0$ whenever $\left(i_{k+1}, j_{k+1}\right) \preceq(s, t)$.

Proof. Equation (1.22) is equivalent to

$$
\begin{aligned}
A_{\left(i_{k+1}, j_{k+1}\right),(s, t)}= & \sum_{\substack{\alpha i_{k}+m=s \\
\alpha j_{k}+n=t \\
(m, n) \neq(0,0),\left(i_{k}, j_{k}\right)}}(-1)^{\alpha} A_{\left(i_{k}, j_{k}\right),\left(i_{k}, j_{k}\right)}^{\alpha} A_{\left(i_{k}, j_{k}\right),(m, n)} \\
+ & (-A)_{\left(i_{k}, j_{k}\right),\left(i_{k}, j_{k}\right)}^{\frac{s}{i_{k}}}+(-1)^{\frac{s}{i_{k}}-1}(A)_{\left(i_{k}, j_{k}\right),\left(i_{k}, j_{k}\right)}^{\frac{s}{i_{k}}} .
\end{aligned}
$$

Rewrite Equation (1.23) as $A_{\left(i_{k+1}, j_{k+1}\right),(s, t)}=\beta+\gamma$, where

$$
\beta:=\sum_{\begin{array}{c}
\alpha i_{k}+m=s \\
\alpha j_{k}+n=t \\
(m, n) \neq(0,0),\left(i_{k}, j_{k}\right)
\end{array}}(-1)^{\alpha} A_{\left(i_{k}, j_{k}\right),\left(i_{k}, j_{k}\right)}^{\alpha} A_{\left(i_{k}, j_{k}\right),(m, n)},
$$


and

$$
\gamma:=(-A)_{\left(i_{k}, j_{k}\right),\left(i_{k}, j_{k}\right)}^{\frac{s}{i_{k}}}+(-1)^{\frac{s}{i_{k}}-1}(A)_{\left(i_{k}, j_{k}\right),\left(i_{k}, j_{k}\right)}^{\frac{s}{i_{k}}} .
$$

By the hypothesis, $A_{\left(i_{k}, j_{k}\right),\left(i_{k}, j_{k}\right)}^{\alpha} A_{\left(i_{k}, j_{k}\right),(m, n)}$ is the product of $\alpha+1$ non-positive numbers and is either zero or has a sign of $(-1)^{\alpha+1}$. Thus

$(-1)^{\alpha} A_{\left(i_{k}, j_{k}\right),\left(i_{k}, j_{k}\right)}^{\alpha} A_{\left(i_{k}, j_{k}\right),(m, n)}$ is either zero or negative, and each summand in $\beta$ is non-positive.

It remains to show that $\gamma$ is also non-positive. Note that $\gamma$ only exists if $\alpha=\frac{s}{i_{k}}$ is a positive integer, say $\frac{s}{i_{k}}=\widehat{\alpha}>1$. Then $\gamma$ becomes

$$
\gamma=(-A)_{\left(i_{k}, j_{k}\right),\left(i_{k}, j_{k}\right)}^{\widehat{\alpha}}+(-1)^{\widehat{\alpha}-1}(A)_{\left(i_{k}, j_{k}\right),\left(i_{k}, j_{k}\right)}^{\widehat{\alpha}}=0 .
$$

Therefore, the representation of $\gamma$ provided by Equation (1.24) shows that $\gamma$ is non-positive.

It will be convenient to insert a definition before stating the main result in this section.

Definition 1.3.1. The symbol $\phi=\left(\left(i_{1}, j_{1}\right),\left(i_{2}, j_{2}\right), \ldots,\left(i_{n}, j_{n}\right)\right)$ stands for a vector with $n$ components, where $n \in \mathbb{N}$ and $i_{1}, i_{2}, \ldots, i_{n}, j_{1}, j_{2}, \ldots, j_{n} \in \mathbb{N}_{0}$. Let $\tau=\tau(\phi)$ be the length of $\phi$, i.e. $\tau=n$. Let $|\phi|$ denote the sum of the components, i.e. $|\phi|=\left(\sum_{d=1}^{n} i_{d}, \sum_{d=1}^{n} j_{d}\right)$. We denote the symbol $A_{\left(i_{k}, j_{k}\right), \phi}$ as $A_{\left(i_{k}, j_{k}\right),\left(i_{1}, j_{1}\right)} A_{\left(i_{k}, j_{k}\right),\left(i_{2}, j_{2}\right)} \ldots A_{\left(i_{k}, j_{k}\right),\left(i_{n}, j_{n}\right)}$.

The following example illustrates the meaning of Definition 1.3.1.

Example 1.3.1. Let $\phi=((2,2),(1,3),(4,0),(2,2),(0,4))$, then $\tau=\tau(\phi)=5$, $|\alpha|=(9,11)$, and

$$
A_{\left(i_{k}, j_{k}\right),((2,2),(1,3),(4,0),(2,2),(0,4))}=A_{\left(i_{k}, j_{k}\right),(2,2)}^{2} A_{\left(i_{k}, j_{k}\right),(1,3)} A_{\left(i_{k}, j_{k}\right),(4,0)} A_{\left(i_{k}, j_{k}\right),(0,4)} .
$$


Theorem 1.3.2. (Structure Property) Let $\left(i_{k}, j_{k}\right) \in \mathbb{N}_{0} \times \mathbb{N}_{0} \backslash\{(0,0)\}$. Then

$$
\begin{aligned}
A_{\left(i_{k+1}, j_{k+1}\right),(s, t)} & =\sum_{\eta}(-1)^{\tau(\phi(\eta))-1} A_{\left(i_{k}, j_{k}\right), \phi(\eta)} \\
& =\sum_{\eta}(-1)^{\tau(\phi(\eta))+1} A_{\left(i_{k}, j_{k}\right), \phi(\eta)},
\end{aligned}
$$

where the sum is over all $\phi(\eta)=\left(\left(i_{1}, j_{1}\right),\left(i_{2}, j_{2}\right), \ldots,\left(i_{\tau}, j_{\tau}\right)\right)$ such that $|\phi(\eta)|=(s, t)$ and at most one component $\left(l_{\theta}, l_{\theta}^{\prime}\right) \neq\left(i_{k}, j_{k}\right)$, where $1 \leq \theta \leq \tau$. Moreover, define $A_{\left(i_{k}, j_{k}\right), \phi(\eta)}=A_{\left(i_{k}, j_{k}\right),\left(i_{1}, j_{1}\right)} \ldots A_{\left(i_{k}, j_{k}\right),\left(i_{\tau}, j_{\tau}\right)}$. If $A_{\left(i_{k}, j_{k}\right),(s, t)} \leq 0$ and all $\left(i_{k}, j_{k}\right) \preceq(s, t)$, then Equation (1.25) is equivalent to

$$
A_{\left(i_{k+1}, j_{k+1}\right),(s, t)}=-\sum_{\eta}\left|A_{\left(i_{k}, j_{k}\right),\left(i_{1}, j_{1}\right)}\right| \ldots\left|A_{\left(i_{k}, j_{k}\right),\left(i_{\tau}, j_{\tau}\right)}\right|,
$$

where the sum is over all $\phi(\eta)=\left(\left(i_{1}, j_{1}\right),\left(i_{2}, j_{2}\right), \ldots,\left(i_{\tau}, j_{\tau}\right)\right)$ such that $|\phi(\eta)|=(s, t)$ and at most one $\left(l_{\theta}, l_{\theta}^{\prime}\right) \neq\left(i_{k}, j_{k}\right)$, where $1 \leq \theta \leq \tau$.

Proof. We obtain the desired result by representing $A_{\left(i_{k}, j_{k}\right),\left(i_{k}, j_{k}\right)}^{\alpha} A_{\left(i_{k}, j_{k}\right),(m, n)}$ in Equation (1.22) as $A_{\left(i_{k}, j_{k}\right), \phi(\eta)}$. Note that $(-1)^{\alpha}=(-1)^{\tau(\phi(\eta))-1}$.

Let us see what happens when we repeat Equation (1.26). In order to efficiently record the results, let $\phi=\left(\left(i_{1}, j_{1}\right),\left(i_{2}, j_{2}\right), \ldots,\left(i_{n}, j_{n}\right)\right)$ denote a vector with $n$ components, where $n \in \mathbb{N}$, and $i_{1}, i_{2}, \ldots, i_{n}, j_{1}, j_{2}, \ldots, j_{n} \in \mathbb{N}_{0}$. Then $a_{\phi(\eta)}$ denotes the expression $a_{i_{1}, j_{1}} a_{i_{2}, j_{2}} \ldots a_{i_{n}, j_{n}}$. After $L$ iterations, and assuming $A_{\left(i_{k}, j_{k}\right),(s, t)} \leq 0$, whenever $\left(i_{k}, j_{k}\right) \preceq(s, t)$, we obtain

$$
A_{\left(i_{k+1}, j_{k+1}\right),(s, t)}=\sum_{\eta}(-1)^{\tau(\phi(\eta))-1} a_{\phi(\eta)}=-\sum_{\eta}\left|a_{i_{1}, j_{1}}\right|\left|a_{i_{2}, j_{2}}\right|,,,\left|a_{i_{\tau}, j_{\tau}}\right| .
$$

where the sum is over all $\phi(\eta)=\left(\left(i_{1}, j_{1}\right),\left(i_{2}, j_{2}\right), \ldots,\left(i_{\tau}, j_{\tau}\right)\right)$ such that $|\phi(\eta)|=$ $(s, t)$.

If $(s, t)=\left(i_{k+1}, j_{k+1}\right)$, then Equation (1.27) becomes

$$
\begin{aligned}
A_{\left(i_{k+1}, j_{k+1}\right),\left(i_{k+1}, j_{k+1}\right)}=g_{i_{k+1}, j_{k+1}} & =\sum_{\eta}(-1)^{\tau(\phi(\eta))-1} a_{\phi(\eta)}, \\
& =-\sum_{\eta}\left|a_{i_{1}, j_{1}}\right|\left|a_{i_{2}, j_{2}}\right| \ldots\left|a_{i_{\tau}, j_{\tau}}\right| .
\end{aligned}
$$

where the sum is over all $\phi(\eta)=\left(\left(i_{1}, j_{1}\right),\left(i_{2}, j_{2}\right), \ldots,\left(i_{\tau}, j_{\tau}\right)\right)$ such that $|\phi(\eta)|=\left(i_{k+1}, j_{k+1}\right)$. 
The following example illustrates applications of the structure property to calculate $g_{2,1}$ :

$$
\begin{array}{rlr}
g_{2,1}= & A_{(2,1),(2,1)} & \text { By using Equation } \\
= & A_{(3,0),(2,1)} & \text { By using Equation } \\
= & A_{(0,2),(2,1)} \\
= & A_{(1,1),(2,1)} \\
= & A_{(2,0),(2,1)} \\
= & (-1)^{1} A_{(0,1),(0,1)} A_{(0,1),(2,0)}+A_{(0,1),(2,1)} \\
= & (-1)^{1} A_{(1,0),(0,1)} A_{(1,0),(2,0)}+(-1)^{1} A_{(1,0),(1,0)} A_{(1,0),(1,1)}+ \\
& (-1)^{2} A_{(1,0),(1,0)}^{2} A_{(1,0),(0,1)}+A_{(1,0),(2,1)} \\
= & -a_{0,1} a_{2,0}-a_{1,0} a_{1,1}+a_{1,0}^{2} a_{0,1}+a_{2,1} .
\end{array}
$$

By using Equation (1.22)

Below we explicitly list $g_{m, n}$ for $1 \leq m+n \leq 4$.

$$
\begin{aligned}
g_{1,0}= & (-1)^{0} a_{1,0} \\
g_{0,1}= & (-1)^{0} a_{0,1} \\
g_{2,0}= & (-1)^{0} a_{2,0} \\
g_{0,2}= & (-1)^{0} a_{0,2} \\
g_{1,1}= & (-1)^{0} a_{1,1}+(-1)^{1} a_{1,0} a_{0,1} \\
g_{3,0}= & (-1)^{0} a_{3,0}+(-1)^{1} a_{2,0} a_{1,0} \\
g_{2,1}= & (-1)^{0} a_{2,1}+(-1)^{1} a_{0,1} a_{2,0}+(-1)^{1} a_{1,0} a_{1,1}+(-1)^{2} a_{1,0}^{2} a_{0,1} \\
g_{1,2}= & (-1)^{0} a_{1,2}+(-1)^{1} a_{1,0} a_{0,2}+(-1)^{1} a_{0,1} a_{1,1}+(-1)^{2} a_{0,1}^{2} a_{1,0} \\
g_{0,3}= & (-1)^{0} a_{0,3}+(-1)^{1} a_{0,2} a_{0,1} \\
g_{4,0}= & (-1)^{0} a_{4,0}+(-1)^{1} a_{1,0} a_{3,0}+(-1)^{2} a_{2,0} a_{1,0}^{2} \\
g_{3,1}= & (-1)^{0} a_{3,1}+(-1)^{3} a_{1,0}^{3} a_{0,1}+(-1)^{2} a_{1,0}^{2} a_{1,1}+(-1)^{1} a_{0,1} a_{3,0}+(-1)^{1} a_{1,1} a_{2,0} \\
& +(-1)^{1} a_{1,0} a_{2,1}+(-1)^{2} 2 a_{0,1} a_{1,0} a_{2,0} \\
g_{2,2}= & (-1)^{0} a_{2,2}+(-1)^{1} a_{2,0} a_{0,2}+(-1)^{3} 2 a_{1,0}^{2} a_{0,1}^{2}+(-1)^{1} a_{0,1} a_{2,1}+(-1)^{2} a_{0,1}^{2} a_{2,0} \\
& +(-1)^{2} a_{1,0}^{2} a_{0,2}+(-1)^{2} 2 a_{1,0} a_{0,1} a_{1,1}+(-1)^{1} a_{1,0} a_{1,2}+(-1)^{2} a_{1,0} a_{1,0} a_{0,2} \\
g_{1,3}= & (-1)^{0} a_{1,3}+(-1)^{3} a_{0,1}^{3} a_{1,0}+(-1)^{2} a_{0,1}^{2} a_{1,1}+(-1)^{1} a_{1,0} a_{0,3}+(-1)^{1} a_{1,1} a_{0,2} \\
& +(-1)^{1} a_{0,1} a_{1,2}+(-1)^{2} 2 a_{0,1} a_{1,0} a_{0,2}
\end{aligned}
$$


$g_{0,4}=(-1)^{0} a_{0,4}+(-1)^{1} a_{0,1} a_{0,3}+(-1)^{2} a_{0,2} a_{0,1}^{2}$.

\subsection{Convergence Criteria for Power Product Expan- sion}

Our primary purpose in this section is to apply the results of Section 1.3 in order to determine a lower bound for the domain of convergence of the right hand side of Equation (1.7). Since we are dealing with doubly indexed infinite products, we must carefully define what is meant by the convergence $\prod_{\substack{q=1 \\ m+n=q}}^{\infty}\left(1+g_{m, n} x^{m} y^{n}\right)$.

Definition 1.4.1. A PPE2 $\prod_{\substack{q=1 \\ m+n=q}}^{\infty}\left(1+g_{m, n} x^{m} y^{n}\right)$ converges if and only if $\lim _{P \rightarrow \infty} \prod_{\substack{q=1 \\ m+n=q}}^{P}\left(1+g_{m, n} x^{m} y^{n}\right)$ converges to a nonzero complex number. Note that the order of the elementary factors within $\prod_{\substack{q=1 \\ m+n=q}}^{P}\left(1+g_{m, n} x^{m} y^{n}\right)$ follows the conventions of Definition 1.2.1.

We are primarily interested in the absolute convergence of $\prod_{\substack{q=1 \\ m+n=q}}^{\infty}\left(1+g_{m, n} x^{m} y^{n}\right)$. Therefore, following the lead of J. Thunder [43], we make the following definition:

Definition 1.4.2. The PPE2 $\prod_{\substack{q=1 \\ m+n=q}}^{\infty}\left(1+g_{m, n} x^{m} y^{n}\right)$ is absolutely convergent if and only if $\prod_{\substack{q=1 \\ m+n=q}}^{\infty}\left(1+\left|g_{m, n}\right|\left|x^{m} y^{n}\right|\right)$ converges, that is if and only if $\lim _{m, n \rightarrow \infty} \prod_{i=1}^{n} \prod_{j=1}^{m}(1+$ $\left.\left|g_{i, j}\right|\left|x^{i} y^{j}\right|\right)$ converges to a nonzero real number. 
Since $e^{x} \geq x+1$ whenever $x \geq 0$, we observe that

$$
\begin{aligned}
\sum_{i=1}^{m} \sum_{j=1}^{n}\left|g_{i, j}\right|\left|x^{i} y^{j}\right| & <\prod_{i=1}^{m} \prod_{j=1}^{n}\left(1+|| g_{i, j}||\left|x^{i} y^{j}\right|\right) \\
& \leq \prod_{i=1}^{m} \prod_{j=1}^{n} e^{\left|g_{i, j}\right|\left|x^{i} y^{j}\right|}=e^{\sum_{i=1}^{m} \sum_{j=1}^{n}\left|g_{i, j}\right|\left|x^{i} y^{j}\right|} .
\end{aligned}
$$

Thus Lemma 2 of [43] is applicable and we have the following proposition:

Proposition 1.4.1. A PPE2 $\prod_{\substack{q=1 \\ m+n=q}}^{\infty}\left(1+g_{m, n} x^{m} y^{n}\right)$ is absolutely convergent if and only

if $\sum_{\substack{q=1 \\ m+n=q}}^{\infty}\left|g_{m, n}\right|\left|x^{m} y^{n}\right|$ is an absolutely convergent series of real numbers.

As it the case of a double series, if a PPE2 $\prod_{\substack{q=1 \\ m+n=q}}^{\infty}\left(1+g_{m, n} x^{m} y^{n}\right)$ is absolutely convergent, then order of multiplication is immaterial and thus we choose to apply the ordering of Definition 1.2.1. We implicitly made use of this fact in Proposition 1.4.1.

if $\left(g_{m, n}\right)$ is a sequence of complex numbers, we define

$$
\log \left(\prod_{\substack{q=1 \\ m+n=q}}^{\infty}\left(1+g_{m, n} x^{m} y^{n}\right)\right):=\sum_{\substack{q=1 \\ m+n=q}}^{\infty} \log \left(1+g_{m, n} x^{m} y^{n}\right),
$$

where

$$
\log \left(1+g_{m, n} x^{m} y^{n}\right):=\sum_{\ell=1}^{\infty}(-1)^{\ell} \frac{g_{m, n}^{\ell}}{\ell} x^{m \ell} y^{n \ell} .
$$

Equation (1.29) implies that

$$
\begin{aligned}
\exp \left(\sum_{\substack{q=1 \\
m+n=q}}^{\infty} \log \left(1+g_{m, n} x^{m} y^{n}\right)\right): & =\exp \left[\log \left(\prod_{\substack{q=1 \\
m+n=q}}^{\infty}\left(1+g_{m, n} x^{m} y^{n}\right)\right)\right] \\
& =\prod_{\substack{q=1 \\
m+n=q}}^{\infty}\left(1+g_{m, n} x^{m} y^{n}\right),
\end{aligned}
$$


and implies that for a sequence of complex coefficients $\left(g_{m, n}\right)$, the PPE2 $\prod_{\substack{q=1 \\ m+n=q}}^{\infty}\left(1+g_{m, n} x^{m} y^{n}\right)$ will be absolutely convergent if and only if the double series

$$
\sum_{\substack{q=1 \\ m+n=q}}^{\infty} \log \left(1+g_{m, n} x^{m} y^{n}\right):=\sum_{\substack{q=1 \\ m+n=q}}^{\infty} \sum_{\ell=1}^{\infty}(-1)^{\ell} \frac{g_{m, n}^{\ell}}{\ell} x^{m \ell} y^{n \ell}
$$

is absolutely convergent, i.e. if and only if

$$
\sum_{\substack{q=1 \\ m+n=q}}^{\infty} \sum_{\ell=1}^{\infty} \frac{\left|g_{m, n}^{\ell}\right|}{\ell}|x|^{m \ell}|y|^{n \ell}<\infty
$$

By adapting the Taylor series argument found on Page 165 of [5], we have

$$
1 / 2|\Delta| \leq|\log (1+\Delta)| \leq 3 / 2|\Delta|, \quad|\Delta|<1 / 2 .
$$

(In our case $\Delta=g_{m, n} x^{m} y^{n}$.) Equation (1.32) implies that $\sum_{\substack{q=1 \\ m+n=q}}^{\infty} \log \left(1+g_{m, n} x^{m} y^{n}\right)$ is absolutely convergent if and only if $\sum_{\substack{q=1 \\ m+n=q}}^{\infty} g_{m, n} x^{m} y^{n}$ is absolutely convergent, a fact we record in the following Theorem:

Theorem 1.4.1. Let $f(x, y)=1+\sum_{\substack{p=1 \\ m+n=p}}^{\infty} a_{m, n} x^{m} y^{n}$. Then $f(x, y)$ is represented by the PPE2

$$
f(x, y)=1+\sum_{\substack{p=1 \\ m+n=p}}^{\infty} a_{m, n} x^{m} y^{n}=\prod_{\substack{q=1 \\ m+n=q}}^{\infty}\left(1+g_{m, n} x^{m} y^{n}\right) .
$$

Consider the following auxiliary functions:

$$
\begin{aligned}
& C(x, y)=1-\sum_{\substack{p=1 \\
m+n=p}}^{\infty}\left|a_{m, n}\right| x^{m} y^{n}=\prod_{\substack{q=1 \\
m+n=q}}^{\infty}\left(1-\widehat{G}_{m, n} x^{m} y^{n}\right) \\
& D(x, y)=1-\sum_{\substack{p=1 \\
m+n=p}}^{\infty} A_{m, n} x^{m} y^{n}=\prod_{\substack{q=1 \\
m+n=q}}^{\infty}\left(1-E_{m, n} x^{m} y^{n}\right) .
\end{aligned}
$$

Assume $0 \leq\left|a_{m, n}\right| \leq A_{m, n}$ for all $(n, m) \in \mathbb{N}_{0} \times \mathbb{N}_{0} \backslash\{(0,0)\}$. Then $\left|g_{m, n}\right| \leq \widehat{G}_{m, n} \leq E_{m, n}$ for all $(n, m) \in \mathbb{N}_{0} \times \mathbb{N}_{0} \backslash\{(0,0)\}$. 
Proof. By Equation (1.28) we have

$$
g_{m, n}=\sum_{\substack{\eta \\|\phi(\eta)|=(m, n)}}(-1)^{\tau(\phi(\eta))-1} a_{\phi(\eta)}=\sum_{\substack{\eta \\|\phi(\eta)|=(m, n)}}(-1)^{\tau(\phi(\eta))-1} a_{i_{1}, j_{1}} a_{i_{2}, j_{2}} \ldots a_{i_{\tau}, j_{\tau}} .
$$

Observe that Equation (1.36) implies that

$$
\begin{aligned}
\left|g_{m, n}\right| & =\left|\sum_{\substack{\eta \\
|\phi(\eta)|=(m, n)}}(-1)^{\tau(\phi(\eta))-1} a_{i_{1}, j_{1}} a_{i_{2}, j_{2}} \ldots a_{i_{\tau}, j_{\tau}}\right| \\
& \leq \sum_{\substack{\eta \\
|\phi(\eta)|=(m, n)}}\left|a_{i_{1}, j_{1}}\right|\left|a_{i_{2}, j_{2}}\right| \ldots\left|a_{i_{\tau}, j_{\tau}}\right| .
\end{aligned}
$$

Similarly, when we apply Equation (1.28) to Equation (1.34), we obtain

$$
\begin{aligned}
0 \leq \widehat{G}_{m, n} & =\sum_{\substack{\eta \\
|\phi(\eta)|=(m, n)}}(-1)^{\tau(\phi(\eta))}\left(-\left|a_{i_{1}, j_{1}}\right|\right)\left(-\left|a_{i_{2}, j_{2}}\right|\right) \ldots\left(-\left|a_{i_{\tau}, j_{\tau}}\right|\right) \\
& =\sum_{\substack{\eta \\
|\phi(\eta)|=(m, n)}}(-1)^{\tau(2 \phi(\eta))}\left(\left|a_{i_{1}, j_{1}}\right|\right)\left(\left|a_{i_{2}, j_{2}}\right|\right) \ldots\left(\left|a_{i_{\tau}, j_{\tau}}\right|\right) \\
& =\sum_{\substack{\eta \\
|\phi(\eta)|=(m, n)}}\left|a_{i_{1}, j_{1}}\right|\left|a_{i_{2}, j_{2}}\right| \ldots\left|a_{i_{\tau}, j_{\tau}}\right| .
\end{aligned}
$$

By combining Equations (1.37) and (1.38), we deduce that $\left|g_{m, n}\right| \leq \widehat{G}_{m, n}$. Also, due to the inequality $\left|a_{m, n}\right| \leq A_{m, n}$, we have

$$
\begin{aligned}
0 \leq \widehat{G}_{m, n} & =\sum_{\substack{\eta \\
|\phi(\eta)|=(m, n)}}\left|a_{i_{1}, j_{1}}\right|\left|a_{i_{2}, j_{2}}\right| \ldots\left|a_{i_{\tau}, j_{\tau}}\right| \\
& \leq \sum_{\substack{\eta \\
|\phi(\eta)|=(m, n)}} A_{i_{1}, j_{1}} A_{i_{2}, j_{2}} \ldots A_{i_{\tau}, j_{\tau}}=E_{m, n} .
\end{aligned}
$$

where the last equality follows from Equation (1.28).

Remark 1.4.1. Theorem 3.1, Part i, of [17] and Theorem 3.1 of [22] are special cases of Theorem 1.4.1.

We now consider the special case for $D(x, y)$, particularly

$$
D(x, y)=1-\sum_{\substack{p=1 \\ m+n=p}}^{\infty} s^{m+n} x^{n} y^{n}=\prod_{\substack{q=1 \\ m+n=q}}^{\infty}\left(1-E_{m, n} x^{m} y^{n}\right)
$$


where $s: \equiv \sup _{\substack{p=1 \\ m+n=p}}\left|a_{m, n}\right|^{\frac{1}{m+n}}$

Our objective is to find a domain of definition such that the PPE2 of Equation (1.39) is absolutely convergent. Define

$$
\log \left(1-E_{m, n} x^{m} y^{n}\right):=-\sum_{\ell=1}^{\infty} \frac{\left(E_{m, n} x^{m} y^{n}\right)^{\ell}}{\ell} .
$$

Next define

$$
\sum_{\substack{p=1 \\ m+n=p}}^{\infty} \log \left(1-E_{m, n} x^{m} y^{n}\right):=-\sum_{\substack{p=1 \\ m+n=p}}^{\infty} \sum_{\ell=1}^{\infty} \frac{\left(E_{m, n} x^{m} y^{n}\right)^{\ell}}{\ell}
$$

The equality in (1.40) tells us that absolute convergence of the double series on the right hand side implies that both $\sum_{\substack{p=1 \\ m+n=p}}^{\infty} \log \left(1-E_{m, n} x^{m} y^{n}\right)$ and $\log \left(1-E_{m, n} x^{m} y^{n}\right)$ are absolutely convergent. Moreover, since

$$
\exp \left(\sum_{\substack{q=1 \\ m+n=q}}^{\infty} \log \left(1-E_{m, n} x^{m} y^{n}\right)\right)=\prod_{\substack{q=1 \\ m+n=q}}^{\infty}\left(1-E_{m, n} x^{m} y^{n}\right)
$$

the absolute convergence of the double series implies the absolute convergence of $\prod_{\substack{q=1 \\ m+n=q}}^{\infty}\left(1-E_{m, n} x^{m} y^{n}\right)$ as well. Therefore, in order to determine where $\prod_{\substack{q=1 \\ m+n=q}}^{\infty}\left(1-E_{m, n} x^{m} y^{n}\right)$ will be absolutely convergent, it suffices to consider the absolutely convergence of $\sum_{\substack{q=1 \\ m+n=q}}^{\infty} \log \left(1-E_{m, n} x^{m} y^{n}\right)$. Taking the logarithm of the both sides of Equation (1.39) gives

$$
\begin{aligned}
\log \left(1-\sum_{\substack{p=1 \\
m+n=p}}^{\infty} s^{m+n} x^{m} y^{n}\right) & =\log \prod_{\substack{q=1 \\
m+n=q}}^{\infty}\left(1-E_{m, n} x^{m} y^{n}\right) \\
& =\sum_{\substack{q=1 \\
m+n=q}}^{\infty} \log \left(1-E_{m, n} x^{m} y^{n}\right) .
\end{aligned}
$$


Observe that

$$
\begin{aligned}
1-\sum_{\substack{p=1 \\
m+n=p}}^{\infty} s^{m+n} x^{m} y^{n} & =1-\left(\sum_{m=0}^{\infty}(s x)^{m} \sum_{n=0}^{\infty}(s y)^{n}-1\right) \\
& =1-\left(\frac{1}{1-s x} \cdot \frac{1}{1-s y}-1\right)=\frac{1-2 s(x+y)+2 s^{2} x y}{(1-s x)(1-s y)} .
\end{aligned}
$$

This implies that

$$
\begin{aligned}
& \log \left(\frac{1-2 s(x+y)+2 s^{2} x y}{(1-s x)(1-s y)}\right) \\
& =\log \left(1-\left[2 s(x+y)-2 s^{2} x y\right]\right)-\log (1-s x)-\log (1-s y) \\
& =\sum_{\ell=1}^{\infty} \frac{(s x)^{\ell}}{\ell}+\sum_{\ell=1}^{\infty} \frac{(s y)^{\ell}}{\ell}-\sum_{\ell=1}^{\infty} \frac{\left[2 s(x+y)-2 s^{2} x y\right]^{\ell}}{\ell} .
\end{aligned}
$$

The three series in (1.42) are absolutely convergent for $|x|<\frac{1}{s},|y|<\frac{1}{s}$, and for $\left|2 s(x+y)-2 s^{2} x y\right|<1$, respectively. By triangular inequality, we have

$$
\left|2 s(x+y)-2 s^{2} x y\right| \leq 2 s(|x|+|y|)+2 s^{2}|x||y| \text {. }
$$

If we require $2 s(|x|+|y|)+2 s^{2}|x||y|<1$, since $2 s|x|<2 s(|x|+|y|)+2 s^{2}|x||y|<1$, we find that $|x|<\frac{1}{2 s}$. Similarly, $|y|<\frac{1}{2 s}$.

Therefore, the estimate of a convergence domain of (1.42) is given by

$$
\mathbb{D}=\left\{(x, y) \in \mathbb{C}^{2}: 2 s(|x|+|y|)+2 s^{2}|x||y|<1\right\} .
$$

See Figure 1.2.

Relation (1.43) makes it possible to also obtain a domain of absolute convergence in terms of polydiscs. Let $|x|<\rho$ and $|y|<\rho$. Inequality (1.43) implies that the PPE2 will be absolutely convergent if

$$
2 s(2 \rho)+2 s^{2} \rho^{2}=2\left[(s \rho+1)^{2}-1\right]<1,
$$

or equivalently if $\rho<s^{-1}\left[\sqrt{\frac{3}{2}}-1\right]$. The inequalities for $\rho$ obtained from the defining quadratic equation of $\mathbb{D}$, namely

$$
|x|<s^{-1}\left[\sqrt{\frac{3}{2}}-1\right], \quad|y|<s^{-1}\left[\sqrt{\frac{3}{2}}-1\right],
$$


are sharp in the sense that if

$$
x=-s^{-1}\left[\sqrt{\frac{3}{2}}-1\right], \quad y=-s^{-1}\left[\sqrt{\frac{3}{2}}-1\right],
$$

then $\left|2 s(x+y)-2 s^{2} x y\right|=1$ and the sum of the absolute values of the terms in the logarithmic power series of (1.42) diverge.

In summary, we have shown that $\sum_{\substack{q=1 \\ m+n=q}}^{\infty} \log \left(1-E_{m, n} x^{m} y^{n}\right)$ will be absolutely convergent whenever $(x, y) \in \mathbb{D}$ or whenever $(x, y) \in D_{x \rho} \times D_{y \rho}$ with $\rho<s^{-1}\left[\sqrt{\frac{3}{2}}-1\right]$, where

$$
D_{x \rho}: \equiv\{x:|x|<\rho\}, \quad D_{y \rho}: \equiv\{y:|y|<\rho\} .
$$

Hence, $\prod_{\substack{q=1 \\ m+n=q}}^{\infty}\left(1-E_{m, n} x^{m} y^{n}\right)$ will also be absolutely convergent for the same regions. We claim this information provides a lower bound on the range of absolute convergence for the PPE2 of Equation (1.33). To determine the domain of convergence of the PPE2 of Equation (1.33), we must determine the domain of convergence of

$\log \prod_{\substack{q=1 \\ m+n=q}}^{\infty}\left(1+g_{m, n} x^{m} y^{n}\right):=\sum_{\substack{q=1 \\ m+n=q}}^{\infty} \log \left(1+g_{m, n} x^{m} y^{n}\right)$, where the right hand side is defined via the convergence of the double series $\sum_{\substack{q=1 \\ m+n=q}}^{\infty} \sum_{\ell=1}^{\infty} \frac{(-1)^{\ell-1}\left(g_{m, n} x^{m} y^{n}\right)^{\ell}}{\ell}$. However,

$$
\begin{aligned}
\left|\log \prod_{\substack{q=1 \\
m+n=q}}^{\infty}\left(1+g_{m, n} x^{m} y^{n}\right)\right| & =\left|\sum_{\substack{q=1 \\
m+n=q}}^{\infty} \log \left(1+g_{m, n} x^{m} y^{n}\right)\right| \\
& \leq \sum_{\substack{q=1 \\
m+n=q}}^{\infty}\left|\log \left(1+g_{m, n} x^{m} y^{n}\right)\right| \\
& =\sum_{\substack{q=1 \\
m+n=q}}^{\infty}\left|\sum_{\ell=1}^{\infty} \frac{(-1)^{\ell-1}\left(g_{m, n} x^{m} y^{n}\right)^{\ell}}{\ell}\right| \\
& \leq \sum_{\substack{q=1 \\
m+n=q}}^{\infty} \sum_{\ell=1}^{\infty} \frac{\left(\left|g_{m, n}\right||x|^{m}|y|^{n}\right)^{\ell}}{\ell}
\end{aligned}
$$




$$
\leq \sum_{\substack{q=1 \\ m+n=q}}^{\infty} \sum_{\ell=1}^{\infty} \frac{\left(E_{m, n}|x|^{m}|y|^{n}\right)^{\ell}}{\ell}
$$

where the last inequality follows from Theorem (1.4.1). Inequality (1.44) shows that if $\sum_{\substack{p=1 \\ m+n=p}}^{\infty} \sum_{\ell=1}^{\infty} \frac{\left(E_{m, n} x^{m} y^{n}\right)^{\ell}}{\ell}$, and hence $\sum_{\substack{q=1 \\ m+n=q}}^{\infty} \log \left(1-E_{m, n} x^{m} y^{n}\right)$ are absolutely convergent, then $\sum_{\substack{p=1 \\ m+n=p}}^{\infty} \log \left(1+g_{m, n} x^{m} y^{n}\right)$ and $\log \prod_{\substack{q=1 \\ m+n=q}}^{\infty}\left(1+g_{m, n} x^{m} y^{n}\right)$ will be absolutely convergent as well.

We can therefore summarize what we have shown so far in the following theorem.

Theorem 1.4.2. $\quad$ (i) Let $f(x, y)=1+\sum_{\substack{p=1 \\ m+n=p}}^{\infty} a_{m, n} x^{m} y^{n}$. Define

$$
\begin{aligned}
& s: \equiv \sup _{\substack{p=1 \\
m+n=p}}\left|a_{m, n}\right|^{\frac{1}{m+n}} \text {. Then both } f(x, y) \text { and its PPE2, } \\
& \qquad f(x, y)=1+\sum_{\substack{p=1 \\
m+n=p}}^{\infty} a_{m, n} x^{m} y^{n}=\prod_{\substack{q=1 \\
m+n=q}}^{\infty}\left(1+g_{m, n} x^{m} y^{n}\right),
\end{aligned}
$$

and the auxiliary function, along with its PPE2

$$
D(x, y)=1-\sum_{\substack{p=1 \\ m+n=p}}^{\infty} s^{m+n} x^{m} y^{n}=\prod_{\substack{q=1 \\ m+n=q}}^{\infty}\left(1-E_{m, n} x^{m} y^{n}\right)
$$

will be absolutely convergent whenever $(x, y) \in \mathbb{D}$, where

$$
\mathbb{D}=\left\{(x, y) \in \mathbb{C}^{2}: 2 s(|x|+|y|)+2 s^{2}|x||y|<1\right\} .
$$

See Figure 1.2.

(ii) With the same conventions as in Part (i.), both $f(x, y)$ and its PPE2, along with $D(x, y)$ and its PPE2, will be absolutely convergent whenever $(x, y) \in$ $D_{x \rho} \times D_{y \rho}$ with $\rho<s^{-1}\left[\sqrt{\frac{3}{2}}-1\right]$, where

$$
D_{x \rho}: \equiv\{x:|x|<\rho\}, \quad D_{y \rho}: \equiv\{y:|y|<\rho\} .
$$




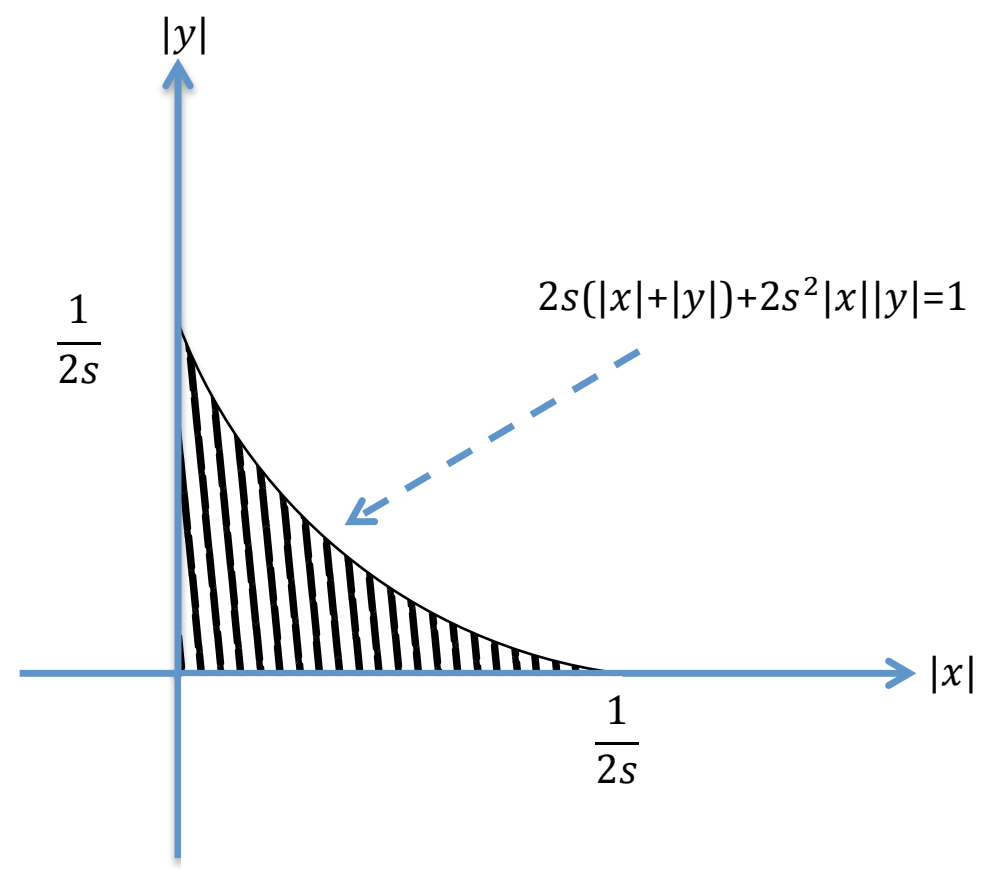

Figure 1.2: A domain of absolute convergence of Equations (2.4.2) and (2.4.2).

\subsection{Asymptotic Approximation for a Special Power Product Expansion}

We will now derive an asymptotic representation for the majorizing product expansion associated with $1-\sum_{\substack{p=1 \\ m+n=p}}^{\infty} s^{m+n} x^{n} y^{n}, \quad$ where $\quad s: \equiv \sup _{\substack{p=1 \\ m+n=p}}\left|a_{m, n}\right|^{\frac{1}{m+n}}$.

The following lemma will be used in the derivation of asymptotic formula.

Lemma 1.5.1. Let $M$ be fixed element of $\mathbb{N}, N, q \in \mathbb{N}$, and $N \leq q \leq M+N$. As $N \rightarrow \infty$, we have

1. $q \sim N$

2. $\left(\begin{array}{c}q \\ M\end{array}\right) \sim \frac{N^{M}}{M !}$. 
Proof. The inequality $N \leq q \leq M+N$ implies that $1 \leq \frac{q}{N} \leq 1+\frac{M}{N}$. Hence, for fixed $M, q \sim N$ as $N \rightarrow \infty$.

To prove the second statement, observe that

$$
\begin{aligned}
\left(\begin{array}{c}
q \\
M
\end{array}\right) & =\frac{\overbrace{q(q-1)(q-2) \ldots(q-M+1)}^{M}}{M !}=\frac{1}{M !}\left[q^{M}+O\left(q^{M-1}\right)\right] \\
& =\frac{q^{M}}{M !}\left[1+\Delta_{2}(q)\right]
\end{aligned}
$$

where $\Delta_{2}(q)=\frac{O\left(q^{M-1}\right)}{q^{M}} \rightarrow 0$ as $q \rightarrow \infty$. From Part 1 , since $q \sim N$ as $N \rightarrow \infty$, the result follows.

Let $(M, N) \in \mathbb{N} \times \mathbb{N}$. Arrange $\left(g_{M, N}\right)$ in an infinite array as shown in Table 1.1. Then for fixed $M \in \mathbb{N}$, the limit $\lim _{N \rightarrow \infty} g_{M, N}$ is along the $M$-th row of Table 1.1.

$\begin{array}{cccc}g_{1,1} & g_{1,2} & g_{1,3} & \cdots \\ g_{2,1} & g_{2,2} & g_{2,3} & \cdots \\ g_{3,1} & g_{3,2} & g_{3,3} & \cdots \\ g_{4,1} & g_{4,2} & g_{4,3} & \cdots \\ \vdots & \vdots & \vdots & \ddots\end{array}$

Table 1.1: The row asymptotics are calculated in Theorem 1.5.1.

Theorem 1.5.1. Let $f(x, y)=1-\sum_{\substack{p=1 \\ m+n=p}}^{\infty} s^{m+n} x^{m} y^{n}=\frac{1-2 s(x+y)+2 s^{2} x y}{(1-s x)(1-s y)}$ where $s>0$. For this special function $f(x, y)$ and its associated PPE2 $\prod_{\substack{p=1 \\ M+N=p}}^{\infty}\left(1+g_{M, N} x^{M} y^{N}\right)$, we have

$$
-g_{M, N} \sim s^{M+N} \sum_{q=N}^{N+M} \frac{2^{q}(q-1) !(-1)^{M+N-q}}{(q-M) !(q-N) !(M+N-q) !}, \quad \text { as } \quad N \rightarrow \infty
$$

where $M$ is a fixed element of $\mathbb{N}$ and $(M, N) \in \mathbb{N} \times \mathbb{N}$. 
Proof. Let

$$
f(x, y)=1+\sum_{\substack{p=1 \\ m+n=p}}^{\infty} a_{m, n} x^{m} y^{n}=\prod_{\substack{q=1 \\ m+n=q}}^{\infty}\left(1+g_{m, n} x^{m} y^{n}\right) .
$$

Define

$$
\log (f(x, y))=\log \left(1+\sum_{\substack{p=1 \\ m+n=p}}^{\infty} a_{m, n} x^{m} y^{n}\right)=\sum_{\substack{p=1 \\ M+N=p}}^{\infty} d_{M, N} x^{M} y^{N} .
$$

Since $f(x, y)=1-\sum_{\substack{p=1 \\ m+n=p}}^{\infty} s^{m+n} x^{m} y^{n}=\frac{1-2 s(x+y)+2 s^{2} x y}{(1-s x)(1-s y)}$, we find that

$$
\begin{aligned}
& \log (f(x, y))=\sum_{\substack{p=1 \\
M+N=p}}^{\infty} d_{M, N} x^{M} y^{N}=\log \left(1-\sum_{\substack{p=1 \\
m+n=p}}^{\infty} s^{m+n} x^{m} y^{n}\right) \\
& =\log \left(\frac{1-2 s(x+y)+2 s^{2} x y}{(1-s x)(1-s y)}\right) \\
& =\log \left(1-\left[2 s(x+y)-2 s^{2} x y\right]\right)-\log (1-s x)-\log (1-s y) \\
& =-\sum_{q=1}^{\infty} \frac{2^{q}\left[s x+s y-s^{2} x y\right]^{q}}{q}+\sum_{q=1}^{\infty} \frac{(s x)^{q}}{q}+\sum_{q=1}^{\infty} \frac{(s y)^{q}}{q} \\
& =-\sum_{q=1}^{\infty} \frac{2^{q}}{q} \sum_{\alpha_{1}+\alpha_{2}+\alpha_{3}=q} \frac{q !(s x)^{\alpha_{1}}(s y)^{\alpha_{2}}\left(-s^{2} x y\right)^{\alpha_{3}}}{\alpha_{1} ! \alpha_{2} ! \alpha_{3} !}+\sum_{q=1}^{\infty} \frac{(s x)^{q}}{q}+\sum_{q=1}^{\infty} \frac{(s y)^{q}}{q} \\
& =-\sum_{q=1}^{\infty} \sum_{\substack{\alpha_{1}, \alpha_{2}=0 \\
q-\alpha_{1}-\alpha_{2} \geq 0}}^{q} \frac{2^{q} q !(-1)^{q-\alpha_{1}-\alpha_{2}}(s x)^{q-\alpha_{2}}(s y)^{q-\alpha_{1}}}{\alpha_{1} ! \alpha_{2} !\left(q-\alpha_{1}-\alpha_{2}\right) ! q}+\sum_{q=1}^{\infty} \frac{(s x)^{q}}{q}+\sum_{q=1}^{\infty} \frac{(s y)^{q}}{q} .
\end{aligned}
$$

If, in the first sum at (1.49), we let $q-\alpha_{2}=M$ and $q-\alpha_{1}=N$, Equation (1.49) becomes

$$
\begin{aligned}
& \log (f(x, y))=\sum_{\substack{p=1 \\
M+N=p}}^{\infty} d_{M, N} x^{M} y^{N} \\
& =-\sum_{q=1}^{\infty} s^{M+N} x^{M} y^{N} \sum_{q=\max \{M, N\}}^{N+M} \frac{2^{q}(q-1) !(-1)^{M+N-q}}{(q-M) !(q-N) !(M+N-q) !}+\sum_{q=1}^{\infty} \frac{(s x)^{q}}{q}+\sum_{q=1}^{\infty} \frac{(s y)^{q}}{q} .
\end{aligned}
$$


Our aim is to use the right side of Equation (1.50) to determine an asymptotic formula for $d_{M, N}$ whenever $M \in \mathbb{N}$ is fixed. Since $M$ and $N$ are both non-zero, the coefficient of $d_{M, N}$ depends only on the first sum on the right side of (1.50), namely

$$
d_{M, N}=-s^{M+N} \sum_{q=\max \{M, N\}}^{N+M} \frac{2^{q}(q-1) !(-1)^{M+N-q}}{(q-M) !(q-N) !(M+N-q) !} .
$$

Then for a fixed positive integer $M$, as $N \rightarrow \infty$, Lemma 1.5.1 implies that

$$
\begin{aligned}
d_{M, N} & =-s^{M+N} \sum_{q=N}^{N+M} \frac{2^{q}(q-1) !(-1)^{M+N-q}}{(q-M) !(q-N) !(M+N-q) !} \\
& =-s^{M} s^{N} \sum_{q=N}^{N+M} \frac{2^{N} 2^{q-N}}{N} \cdot \frac{N}{q} \cdot \frac{q !}{(q-M) ! M !} \cdot \frac{M !(-1)^{M}(-1)^{N-q}}{(q-N) !(M+N-q) !} \\
& =\frac{-(-s)^{M}(2 s)^{N}}{N} \sum_{q=N}^{N+M}(-2)^{q-N} \frac{N}{q}\left(\begin{array}{c}
q \\
M
\end{array}\right)\left(\begin{array}{c}
M \\
q-N
\end{array}\right) \\
& =\frac{-(-s)^{M}(2 s)^{N}}{N} \sum_{q=N}^{N+M}(-2)^{q-N}\left(1+\Delta_{1}(q)\right) \frac{N^{M}}{M !}\left(1+\Delta_{2}(q)\right)\left(\begin{array}{c}
M \\
q-N
\end{array}\right) \\
& =-\frac{(-s)^{M}(2 s)^{N} N^{M-1}}{M !} \sum_{q=N}^{N+M}(-2)^{q-N}\left(\begin{array}{c}
M \\
q-N
\end{array}\right)(1+\Delta(q)),
\end{aligned}
$$

where $(1+\Delta(q))=\left(1+\Delta_{1}(q)\right)\left(1+\Delta_{2}(q)\right), \Delta_{1}(q) \rightarrow 0$, and $\Delta_{2}(q) \rightarrow 0$ as $N \rightarrow \infty$. Hence, $\Delta(q) \rightarrow 0$ as $N \rightarrow \infty$.

Denote the right hand side of Equation (1.52) as $T_{1}+T_{2}$, where

$$
\begin{aligned}
& T_{1}:=-\frac{(-s)^{M}(2 s)^{N} N^{M-1}}{M !} \sum_{q=N}^{N+M}(-2)^{q-N}\left(\begin{array}{c}
M \\
q-N
\end{array}\right), \\
& T_{2}:=-\frac{(-s)^{M}(2 s)^{N} N^{M-1}}{M !} \sum_{q=N}^{N+M}(-2)^{q-N}\left(\begin{array}{c}
M \\
q-N
\end{array}\right) \Delta(q) .
\end{aligned}
$$


A change of variable and an application of the binomial theorem shows that

$$
\begin{aligned}
T_{1} & =-\frac{(-s)^{M}(2 s)^{N} N^{M-1}}{M !} \sum_{v=0}^{M}\left(\begin{array}{c}
M \\
v
\end{array}\right)(-2)^{v} \cdot 1^{M-v} \\
& =-\frac{(-s)^{M}(2 s)^{N} N^{M-1}}{M !}(-1)^{M}=-\frac{2^{N} s^{M+N} N^{M-1}}{M !} .
\end{aligned}
$$

Then since $\Delta(q) \rightarrow 0$ as $N \rightarrow \infty$, the closed form of $T_{1}$ implies that

$$
\frac{T_{2}}{T_{1}}=\frac{1}{(-1)^{M}} \sum_{q=N}^{q=N+M}(-2)^{q-N}\left(\begin{array}{c}
M \\
q-N
\end{array}\right) \Delta_{M}(q) \rightarrow 0 \quad \text { as } \quad N \rightarrow \infty,
$$

which means that

$$
d_{M, N}=-\frac{2^{N} s^{M+N} N^{M-1}}{M !}[1+o(1)] \quad \text { as } \quad N \rightarrow \infty .
$$

Equation (1.53) is the desired asymptotic formula for $d_{M, N}$. We now use this formula to obtain an asymptotic formula for $g_{M, N}$ whenever $M \in \mathbb{N}$ is fixed. From Equation (1.12) or (1.14) we have

$$
\overbrace{-g_{M, N}}^{\geq 0}=\overbrace{-d_{M, N}}^{\geq 0}-\underbrace{-\frac{1}{M} \sum_{\substack{m \mid M \\ 1 \leq m \leq\left\lfloor\frac{M}{m}\right\rfloor}} m(\overbrace{-g_{m, \frac{N m}{M}}}^{\geq 0})^{\frac{M}{m}}}_{:=\Delta} \leq-d_{M, N} .
$$

Then as $N \rightarrow \infty$, we have

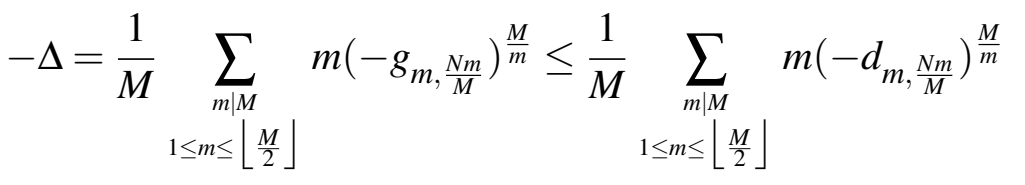

$$
\begin{aligned}
& =\frac{1}{M} \sum_{\substack{m \mid M \\
1 \leq m \leq\left\lfloor\frac{M}{2}\right\rfloor}} m\left[\frac{s^{m}(2 s)^{\frac{N m}{M}}\left(\frac{N m}{M}\right)^{m-1}}{m !}\right]^{\frac{M}{m}}\left[1+\Delta\left(m, \frac{N m}{M}\right)\right]^{\frac{M}{m}} \\
& =\frac{1}{M} \sum_{\substack{m \mid M \\
1 \leq m \leq\left\lfloor\frac{M}{2}\right\rfloor}} \frac{m s^{M}(2 s)^{N}\left(\frac{N m}{M}\right)^{M}}{\left[m !\left(\frac{N m}{M}\right)\right]^{\frac{M}{m}}}\left[1+\Delta\left(m, \frac{N m}{M}\right)\right]^{\frac{M}{m}}
\end{aligned}
$$


where in the penultimate line we used Equation (1.53), which implies that $\Delta\left(m, \frac{N m}{M}\right) \rightarrow 0$ as $N \rightarrow \infty$.

Since

$$
d_{M, N}=-\frac{2^{N} s^{M+N} N^{M-1}}{M !}[1+\Delta(M, N)]
$$

where $\Delta(M, N) \rightarrow 0$ as $N \rightarrow \infty$, we deduce that for fixed $M$, there exist a positive real number $Q(M)$ such that for all $1 \leq m \leq M$

$$
|\Delta(m, N)| \leq Q(M) .
$$

Since the right side inequality (1.57) is independent of $N$, we also have

$$
\left|\Delta\left(m, \frac{N m}{M}\right)\right| \leq Q(M),
$$

whenever $1 \leq m \leq M$. We now return to (1.55) and use (1.58) to discover that for $N \rightarrow \infty$, Hence, as $N \rightarrow \infty$,

$$
\begin{aligned}
\left|\frac{-\Delta}{-d_{M, N}}\right| & =\frac{1}{M} s^{M}(2 s)^{N} \sum_{\substack{m \mid M \\
1 \leq m \leq\left\lfloor\frac{M}{2}\right\rfloor}} \frac{m\left(\frac{N m}{M}\right)^{M}}{\left[m !\left(\frac{N m}{M}\right)\right]^{\frac{M}{m}}} \cdot \frac{M !}{2^{N} s^{M+N} N^{M-1}}\left|\frac{\left[1+\Delta\left(m, \frac{N m}{M}\right)\right]^{\frac{M}{m}}}{1+\Delta(M, N)}\right| \\
& \leq N(M-1) ! \sum_{\substack{m \mid M \\
1 \leq m \leq\left\lfloor\frac{M}{2}\right\rfloor}} \frac{m\left(\frac{m}{M}\right)^{M}}{\left[m !\left(\frac{N m}{M}\right)\right]^{\frac{M}{m}}} \frac{\left[1+\left|\Delta\left(m, \frac{N m}{M}\right)\right|\right]^{\frac{M}{m}}}{1-\Delta(M, N)}
\end{aligned}
$$

Since $\Delta(M, N) \rightarrow 0$ as $N \rightarrow \infty$, we may assume without loss of generality that $|\Delta(M, N)| \leq 1 / 2$ for $N$ large enough. Thus

$$
1-|\Delta(M, N)| \geq 1-\frac{1}{2}, \quad \text { or that } \quad \frac{1}{1-|\Delta(M, N)|} \leq \frac{1}{\frac{1}{2}} .
$$

Returning to (1.59), for $N \rightarrow \infty$, we find that

$$
\left|\frac{-\Delta}{-d_{M, N}}\right| \leq N(M-1) ! \sum_{\substack{m \mid M \\ 1 \leq m \leq\left\lfloor\frac{M}{2}\right\rfloor}} \frac{m\left(\frac{m}{M}\right)^{M}}{\left[m !\left(\frac{N m}{M}\right)\right]^{\frac{M}{m}}} \frac{\left[1+\left|\Delta\left(m, \frac{N m}{M}\right)\right|\right]^{\frac{M}{m}}}{\frac{1}{2}}
$$




$$
\begin{aligned}
& \leq 2 N(M-1) ! \sum_{\substack{m \mid M \\
1 \leq m \leq\left\lfloor\frac{M}{2}\right\rfloor}} \frac{m\left(\frac{m}{M}\right)^{M}}{\left[m !\left(\frac{N m}{M}\right)\right]^{\frac{M}{m}}}[1+Q(M)]^{\frac{M}{m}} \\
& \leq 2 N(M-1) ![1+Q(M)]^{M} \sum_{\substack{m \mid M \\
1 \leq m \leq\left\lfloor\frac{M}{2}\right\rfloor}} \frac{m\left(\frac{m}{M}\right)^{M}}{\left[m !\left(\frac{N m}{M}\right)\right]^{\frac{M}{m}}} \\
& \leq N M(M-1) ![1+Q(M)]^{M} \sum_{\substack{m \mid M \\
1 \leq m \leq\left\lfloor\frac{M}{2}\right\rfloor}} \frac{\left(\frac{m}{M}\right)^{M}}{\left[m !\left(\frac{N m}{M}\right)\right]^{\frac{M}{m}}} \quad \text { since } m \leq \frac{M}{2} \\
& \leq N M !\left(\frac{1}{2}\right)^{M}[1+Q(M)]^{M} \sum_{\substack{m \mid M \\
1 \leq m \leq\left\lfloor\frac{M}{2}\right\rfloor}} \frac{1}{(m !)^{\frac{M}{m}\left(\frac{N m}{M}\right)^{\frac{M}{m}}}} \\
& \leq N M !\left(\frac{1}{2}\right)^{M}[1+Q(M)]^{M} \sum_{\substack{m \mid M \\
1 \leq m \leq\left\lfloor\frac{M}{2}\right\rfloor}} \frac{1}{\left(\frac{N m}{M}\right)^{\frac{M}{m}}} \quad \text { since } \frac{1}{(m !)^{\frac{M}{m}}} \leq 1 \\
& \leq N M !\left(\frac{1}{2}\right)^{M}[1+Q(M)]^{M} \sum_{\substack{m \mid M \\
1 \leq m \leq\left\lfloor\frac{M}{2}\right\rfloor}} \frac{1}{\left(\frac{N}{M}\right)^{\frac{M}{m}}} \\
& \leq N M !\left(\frac{1}{2}\right)^{M}[1+Q(M)]^{M} \sum_{\substack{m \mid M \\
1 \leq m \leq\left\lfloor\frac{M}{2}\right\rfloor}} \frac{1}{\left(\frac{N}{M}\right)^{\frac{M}{M}}} \\
& \leq N M !\left(\frac{1}{2}\right)^{M}[1+Q(M)]^{M} \sum_{\substack{m \mid M \\
1 \leq m \leq\left\lfloor\frac{M}{2}\right\rfloor}} \frac{1}{\left(\frac{N}{M}\right)^{2}} \\
& \leq N M !\left(\frac{1}{2}\right)^{M}[1+Q(M)]^{M} \cdot \frac{M}{2} \cdot \frac{1}{\frac{N^{2}}{M^{2}}}=\frac{M ! M^{3}}{2}\left(\frac{1}{2}\right)^{M}[1+Q(M)]^{M} \cdot \frac{1}{N} \rightarrow 0 .
\end{aligned}
$$

We now return to Equation (1.54) to discover that

$$
\begin{aligned}
-g_{M, N} & =-d_{M, N}-\Delta \\
& =-d_{M, N}\left[1+\frac{\Delta}{d_{M, N}}\right] \rightarrow-d_{M, N} \quad \text { as } \quad N \rightarrow \infty .
\end{aligned}
$$




\subsection{A combinatorial interpretation for PPE2}

The purpose of this section is to discus a combinatorial interpretation for Equation (1.7) involving integer partitions. We begin with a refinement of Definition 1.2.3.

Definition 1.6.1. Let $A \times A \subset \mathbb{N}_{0} \times \mathbb{N}_{0}$. A partition of $(m, n) \in \mathbb{N}_{0} \times \mathbb{N}_{0}$ in $A \times A$ is a collection $\left\{\left(p_{1}, p_{1}^{\prime}\right), \ldots,\left(p_{k}, p_{k}^{\prime}\right)\right\} \subseteq A \times A$ such that $\sum_{i=1}^{k}\left(p_{i}, p_{i}^{\prime}\right)=(m, n)$. The summands or parts $\left(p_{i}, p_{i}^{\prime}\right)$ need not be distinct, and the order of the summands is immaterial. Let $p^{A \times A}((m, n))$ denote the number of all partition of $(m, n)$ in $A \times A$, and $p_{d}^{A \times A}((m, n))$ denote the number of partitions of $(m, n)$ in $A \times A$ with distinct parts.

Example 1.6.1. Let $A \times A=\{(0,0),(1,2),(1,1),(2,3)\}$. Evidently,

$$
\begin{aligned}
& p_{d}^{A \times A}((0,0))=1, \quad p_{d}^{A \times A}((1,2))=1, \quad p_{d}^{A \times A}((1,1))=1, \\
& p_{d}^{A \times A}((2,3))=2, \quad p_{d}^{A \times A}((3,5))=1, \quad p_{d}^{A \times A}((3,4))=1 \text {, } \\
& p_{d}^{A \times A}((4,6))=1,
\end{aligned}
$$

and otherwise $p_{d}^{A \times A}((m, n))=0$. Then we have

$$
\begin{aligned}
\sum_{m+n=0}^{10} p_{d}^{A \times A}((m, n)) x^{m} y^{n} & =1+x^{1} y^{1}+x^{1} y^{2}+2 x^{2} y^{3}+x^{3} y^{5}+x^{3} y^{4}+x^{4} y^{6} \\
& =\left(1+x^{1} y^{1}\right)\left(1+x^{1} y^{2}\right)\left(1+x^{2} y^{3}\right)
\end{aligned}
$$

From this example we deduce that the generating function for the partition of all $(m, n) \in \mathbb{N}_{0} \times \mathbb{N}_{0}$ into distinct parts is given below.

Theorem 1.6.1. (Chapter 12 [1] ) The generating function for the sequence $p_{d}((m, n))$, as defined in Definition 1.2.3, is

$$
\sum_{\substack{p=0 \\ m+n=p}}^{\infty} p_{d}((m, n)) x^{m} y^{n}=\prod_{\substack{q=1 \\ m+n=q}}^{\infty}\left(1+x^{m} y^{n}\right) .
$$

Proof. If we rewrite the right hand side

$$
\prod_{\substack{q=1 \\ m+n=q}}^{\infty}\left(1+x^{m} y^{n}\right)=\left(1+x^{1} y^{0}\right)\left(1+x^{0} y^{1}\right)\left(1+x^{2} y^{0}\right)\left(1+x^{1} y^{1}\right)\left(1+x^{0} y^{2}\right)\left(1+x^{3} y^{0}\right) \ldots
$$


as a power series of the form $\sum_{\substack{p=0 \\ i+j=p}} a_{i, j} x^{i} y^{j}$, then our aim is to show that $p_{d}((i, j))=$ $a_{i, j}$. If multiply the term $x^{1} y^{0}$ from the first parentheses with the term $x^{0} y^{1}$ from the second parentheses and continue this process until we reach the term $x^{\alpha} y^{\beta}$ from the $L-1$ parenthesis, we discover that

$$
x^{i} y^{j}=x^{1} y^{0} x^{0} y^{1} \cdots x^{\alpha} y^{\beta}
$$

which implies that

$$
\begin{aligned}
& i=1+0+\cdots+\alpha, \\
& j=0+1+\cdots+\beta
\end{aligned}
$$

But the last two lines are exactly the definition partition of $(i, j)$ with distinct parts.

The reader might wonder if a similar result holds for arbitrary partitions. Indeed, this is the case as seen by the next theorem.

Theorem 1.6.2. (Chapter 12 [1] ) The generating function for the sequence $p(m, n)$, as defined in Definition 1.2.3, is

$$
\sum_{\substack{p=0 \\ m+n=p}}^{\infty} p((m, n)) x^{m} y^{n}=\prod_{\substack{q=1 \\ m+n=q}}^{\infty}\left(1-x^{m} y^{n}\right)^{-1}
$$

Proof. We expand each factor in the right hand side of Equation (1.62) as a power series to find that

$$
\prod_{\substack{q=1 \\ m+n=q}}^{\infty}\left(1-x^{m} y^{n}\right)^{-1}=\left(1+x+x^{2}+\cdots\right)\left(1+y+y^{2}+\cdots\right)\left(1+x^{2}+x^{4}+\cdots\right) \cdots
$$

The coefficient of the term $x^{m} y^{n}$ is exactly the number of ways to obtain $x^{m} y^{n}$ as a product one term from each infinite sum; i.e. the number of solutions of the equation

$$
x^{m} y^{n}=\left(x^{1} y^{0}\right)^{\alpha_{(1,0)}}\left(x^{0} y^{1}\right)^{\alpha_{(0,1)}}\left(x^{2} y^{0}\right)^{\alpha_{(2,0)}} \ldots\left(x^{i} y^{j}\right)^{\alpha_{(i, j)}}, \text { where } \quad \alpha_{(i, j)} \in \mathbb{N}_{0},
$$


which implies that

$$
\begin{aligned}
(m, n)= & \alpha_{(1,0)}(1,0)+\alpha_{(0,1)}(0,1)+\cdots+\alpha_{(i, j)}(i, j) \\
= & (\overbrace{(1,0)+(1,0)+\cdots+(1,0)}^{\alpha_{(1,0)}-\text { times }})+(\overbrace{(0,1)+(0,1)+\cdots+(0,1)}^{\alpha_{(0,1)}-\text { times }})+\cdots \\
& +(\overbrace{(i, j)+(i, j)+\cdots+(i, j)}^{\alpha_{(i, j)}-\text { times }}) .
\end{aligned}
$$

Thus, the right hand side of the previous equation is a partition of $(m, n)$ into positive summands.

Remark 1.6.1. 1. Observe that the coefficients on the right hand side of (1.63) are non-negative. This is typical to numerous cases in combinatorial interpretations, see e.g. George E. Andrews [1]. It is then important to find out what is the domain of convergence of the right side of (1.63) and compare to the domain of convergence of the left side of (1.63).

2. It is noteworthy that one encounters expansion of a Taylor series $1+\sum_{n=1}^{\infty} a_{n} x^{n}$ that is absolutely convergent in a disk $|x|<R$, but the domain of convergence of corresponding PPE is $R_{1}$, which could be smaller than $R$. For example, the Taylor series of $e^{-x}$ is absolutely convergent for $|x|=$ $R=\infty$ while the corresponding PPE is absolutely convergent for $|x|=R_{1}=$ $1<\infty=R$, see e.g. O. Kolberg, [32]. However, it happens that if the coefficients of the PPE $g_{l}$ in $\prod_{\ell=1}^{\infty}\left(1+g_{\ell} x^{\ell}\right)$ are non-negative then $R_{1}=R$, see H. Gingold, A. Knopfmacher, [19] and H. Gingold and J. Quaintance, [22].

We extend results in [19] and [22] to expansions in two independent variables.

The theory of infinite products is elaborated upon in J.B. Conway [[5], p. 164] and R. Silverman [[42], p. 291]. For the theory of double series see S. Ghorpade, B. Limaye [[28], p. 369]. We will assume that each individual function $g_{\ell}(x, y)$ given in $\prod_{\ell=1}^{\infty}\left(1+g_{\ell}(x, y)\right)$ is a shorthand notation for

$$
g_{\ell}(x, y)=\sum_{m+n=\ell}^{\infty} g_{\ell_{m, n}} x^{m} y^{n}, \quad \ell \in \mathbb{N}
$$


and therefore $\sum_{\ell=1}^{\infty} g_{\ell}(x, y)$ is a shorthand notation for

$$
\sum_{\ell=1}^{\infty} \sum_{m+n=\ell}^{\infty} g_{\ell_{m, n}} x^{m} y^{n}
$$

where the inner sum of (1.64) is actually the double series

$$
\sum_{m+n=\ell}^{\infty} g_{\ell_{m, n}} x^{m} y^{n}:=\sum_{m+n=\ell}^{\infty} \sum_{\eta} g_{\ell_{m, n}} x^{m} y^{n}
$$

where $\eta$ is the sum over all partitions of $(m, n)$ with ordering among the partitions provided by Definition 1.2.1.

If (1.64) is an absolutely convergent double series, we may choose for partial sums any order of summands. Therefore, we choose

$$
\begin{aligned}
& \sum_{\ell=1}^{\infty} \sum_{\ell=m+n}^{\infty} g_{\ell_{m, n}} x^{m} y^{n}=\lim _{L \rightarrow \infty} \sum_{m+n=1}^{L} \sum_{\ell=1}^{m+n} g_{\ell_{m, n}} x^{m} y^{n} \\
& =\left(g_{1_{1,0}} x+g_{1_{0,1}} y\right)+\left(g_{1_{2,0}} x^{2}+g_{1_{1,1}} x y+g_{1_{0,2}} y^{2}+g_{2_{2,0}} x^{2}+g_{2_{1,1}} x y+g_{2_{0,2}} y^{2}\right)+\ldots
\end{aligned}
$$

Furthermore, by adapting the argument of J.B. Conway, [[5], p. 166], we adopt the following definition for the absolute convergence of $\prod_{\ell=1}^{\infty}\left(1+g_{\ell}(x, y)\right)$, namely

Definition 1.6.2. The infinite product $\prod_{\ell=1}^{\infty}\left(1+g_{\ell}(x, y)\right)$ is absolutely convergent in a domain $\mathbb{D}$ if and only if (1.64) is absolutely convergent in $\mathbb{D}$.

With all of these conventions in place, we are ready to prove the following theorem:

Theorem 1.6.3. Let $g_{\ell}(x, y)=\sum_{m+n=\ell}^{\infty} g_{\ell_{m, n}} x^{m} y^{n}$ be an infinite sequence of formal power series with $g_{\ell_{m, n}} \geq 0$ for each $\ell \in \mathbb{N},(m, n) \in \mathbb{N}_{0} \times \mathbb{N}_{0} \backslash\{(0,0)\}$. Suppose that the formal product expansion

$$
f(x, y)=1+\sum_{\substack{p=1 \\ m+n=p}}^{\infty} a_{m, n} x^{m} y^{n}=\prod_{\ell=1}^{\infty}\left(1+g_{\ell}(x, y)\right),
$$


holds in the sense that

$$
a_{m, n}=\sum g_{\ell_{1 m_{1}, n_{1}}} g_{\ell_{2 m_{2}, n_{2}}} \ldots g_{\ell_{r_{r}, n_{r}}},
$$

where the summation is over all $\left(\left(m_{1}, n_{1}\right),\left(m_{2}, n_{2}\right), \ldots,\left(m_{r}, n_{r}\right)\right)$ with

$\left(m_{1}, n_{1}\right)+\left(m_{2}, n_{2}\right)+\cdots+\left(m_{r}, n_{r}\right)=(m, n),(1,0) \preceq\left(m_{1}, n_{1}\right) \preceq\left(m_{2}, n_{2}\right) \preceq \cdots \preceq$ $\left(m_{r}, n_{r}\right)$ and $1 \leq \ell_{1}<\ell_{2}<\cdots<\ell_{r}$. Then $f(x, y)=1+\sum_{\substack{p=1 \\ m+n=p}}^{\infty} a_{m, n} x^{m} y^{n}$ is absolutely convergent in a domain $\mathbb{D}$ if and only if $\prod_{\ell=1}^{\infty}\left(1+g_{\ell}(x, y)\right)$ is absolutely convergent in $\mathbb{D}$. In particular, if $\mathbb{D}=D_{x \rho} \times D_{y \rho}$, where $D_{x \rho}: \equiv\{x:|x|<\rho\}$ and $D_{y \rho}: \equiv$ $\{y:|y|<\rho\}$, then $f(x, y)$ is analytic in the polydiscs $D_{x \rho} \times D_{y \rho}$ if and only if $\prod_{\ell=1}^{\infty}\left(1+g_{\ell}(x, y)\right)$ is analytic in the same $D_{x \rho} \times D_{y \rho}$.

Proof. Assume that $f(x, y)$ is an analytic function whose power series on the left side of (1.65) is absolutely convergent. Note that $a_{m, n}$ in (1.66) is a finite combination of $g_{\ell_{m, n}} \mathrm{~s}$, since $g_{\ell_{m, n}}=0$ for $\ell>m+n$. Furthermore, (1.66) implies that

$$
a_{m, n} \geq \sum_{\ell=1}^{m+n} g_{\ell_{m, n}}
$$

Therefore,

$$
\sum_{\substack{p=1 \\ m+n=p}}^{\infty} a_{m, n} x^{n} y^{n} \geq \sum_{m+n=1}^{\infty} \sum_{\ell=1}^{m+n} g_{\ell_{m, n}} x^{m} y^{n}
$$

The absolute convergence of the left hand side for $(x, y) \in \mathbb{D}$ implies that the right hand side is absolutely convergent. We can now simply interchange the order of summation to get

$$
\infty>\sum_{\substack{p=1 \\ m+n=p}}^{\infty} a_{m, n} x^{n} y^{n} \geq \sum_{\ell=1}^{\infty} \sum_{\ell=m+n}^{\infty} g_{\ell_{m, n}} x^{m} y^{n}=\sum_{\ell=1}^{\infty} g_{\ell}(x, y) .
$$

Hence, $\prod_{\ell=1}^{\infty}\left(1+g_{\ell}(x, y)\right)$ is absolutely convergent for $(x, y) \in \mathbb{D}$.

Conversely, if the product expansion on the right hand side of (1.65) is absolutely convergent, then it defines an analytic function in a domain $\mathbb{D}$. The Taylor 
series expansion of this analytic function coincides with that of $f(x, y)$ and the result follows immediately.

Here are two examples which demonstrate the usefulness of Theorem 1.6.3. First, consider the elementary factors $\left(1+x^{i} y^{j}\right)$ for all $(i, j) \in \mathbb{N}_{0}^{2}$. Then $g_{\ell}(x, y)=x^{m} y^{n}$, where $m+n=\ell$. Evidently, $\sum_{\ell=1}^{\infty} g_{\ell}(x, y)=\sum_{\substack{\ell=1 \\ m+n=\ell}}^{\infty} x^{m} y^{n}$ is absolutely convergent for $|x|<1$ and $|y|<1$, see Example A.3.1 (i). Definition 1.6.2 and Theorem 1.6.3 imply that the domain of convergence of $1+\sum_{\substack{k=1 \\ i+j=k}}^{\infty} p_{d}(i, j) x^{i} y^{j}$ is $\mathbb{D}=\left\{(x, y) \in \mathbb{C}^{2}\right.$ : $|x|<1$ and $|y|<1\}$. For second example, consider the elementary factors $\left(1-x^{i} y^{j}\right)^{-1}$ for all $(i, j) \in \mathbb{N}_{0}^{2}$. Then $g_{\ell}(x, y)=\sum_{\substack{s=1, \ell=1 \\ m+n=\ell}}^{\infty} x^{s m} y^{s n}$ and $\sum_{\ell=1}^{\infty} g_{\ell}(x, y)=$ $\sum_{\substack{\ell=1 \\ m+n=\ell}}^{\infty} \sum_{s=1}^{\infty} x^{s m} y^{s n}$ is absolutely convergent inside the domain $\mathbb{D}=\left\{(x, y) \in \mathbb{C}^{2}:|x|<\right.$ 1 and $|y|<1\}$. Again, Theorem 1.6.3 implies that $1+\sum_{\substack{k=1 \\ i+j=k}}^{\infty} p(i, j) x^{i} y^{j}$ converges inside the domain $\mathbb{D}=\left\{(x, y) \in \mathbb{C}^{2}:|x|<1\right.$ and $\left.|y|<1\right\}$.

Corollary 1.6.1. If

$$
f(x, y)=1+\sum_{\substack{p=1 \\ m+n=p}}^{\infty} a_{m, n} x^{m} y^{n}=\prod_{\substack{q=1 \\ m+n=q}}^{\infty}\left(1+g_{m, n} x^{m} y^{n}\right)
$$

where $g_{m, n} \geq 0$ for $(m, n) \in \mathbb{N}_{0} \times \mathbb{N}_{0}$, then the series and the product have the same domain of absolute convergence.

Proof. Although this result follows immediately from Theorem 1.6.3, we feel it is instructive to provide a direct proof. Assume that $\prod_{\substack{q=1 \\ m+n=q}}^{\infty}\left(1+g_{m, n} x^{m} y^{n}\right)$ is absolutely convergent in domain $\mathbb{D}_{1}$ and defines an analytic function $f$. The Taylor series expansion $1+\sum_{\substack{p=1 \\ m+n=p}}^{\infty} a_{m, n} x^{m} y^{n}$ of this function is absolutely convergent in $(x, y) \in$ $\mathbb{D}_{2} \subseteq \mathbb{D}_{1}$. On the other hand, from Equation (1.10), we have $\sum_{\substack{p=1 \\ m+n=p}}^{\infty} g_{m, n}|x|^{m}|y|^{n} \leq$ $\sum_{\substack{p=1 \\ m+n=p}}^{\infty} a_{m, n}|x|^{m}|y|^{n}$ which implies that $\mathbb{D}_{1} \subseteq \mathbb{D}_{2}$ 
Remark 1.6.2. The preceding theorem provides convergence information for multidimensional partitions as the coefficients $g_{m, n}$ of the corresponding generating function is usually non-negative. 


\section{Chapter 2}

\section{Factorization of Bivariate Taylor Series via Inverse Power Products}

\subsection{Introduction}

Given $f(x, y)=1+\sum_{\substack{p=1 \\ m+n=p}}^{\infty} a_{m, n} x^{m} y^{n}$ with complex coefficients, where either the defining expression for $f(x, y)$ is treated as a formal power series expansion or $f(x, y)$ is an analytic function with $f(0,0)=1$, the right side of

$$
f(x, y)=\prod_{\substack{p=1 \\ m+n=p}}^{\infty}\left(1-h_{m, n} x^{m} y^{n}\right)^{-1}
$$

is defined to be inverse power product expansion in two independent variables, (denoted IPPE2).

This chapter studies expansions of $f(x, y)=1+\sum_{\substack{q=1 \\ m+n=q}}^{\infty} a_{m, n} x^{m} y^{n}$ into its IPPE2

$\prod_{\substack{p=1 \\ m+n=p}}^{\infty}\left(1-h_{m, n} x^{m} y^{n}\right)^{-1}$ and contains algebraic, number theoretic, analytic, asymptotic, and combinatorial results. The three main results are as follows:

1. An algebraic structure property for $\left(h_{m, n}\right)$ in terms of recursive "mixed expansions"; see Theorem 2.3.2. 
2. A domain of convergence criteria for the IPPE2 in terms of a "majorizing" infinite product; see Theorem 2.4.2.

3. An asymptotic approximation for the "majorizing infinite product"; see Theorem 2.5.1.

Theorem 2.3.2 involves expansions of the schematic finite product-series form

$$
f(x, y)=\prod_{\substack{p=1 \\ m+n=p}}^{N}\left(1-h_{m, n} x^{m} y^{n}\right)^{-1}\left[1+\sum A_{m, n} x^{m} y^{n}\right],
$$

where $h_{m, n} \leq 0$ and $A_{m, n} \leq 0$ whenever $a_{m, n} \leq 0$. Ultimately, these finite productseries expansions allow us to write $h_{m, n}$ as a polynomial in the $a_{m, n}$ which preserve the negative sign property; see Equation (2.26). These algebraic properties are crucial for determining a lower bound domain of convergence for the IPPE2. In particular, Theorem 2.4.2 provides a domain of convergence for $f(x, y)=\prod_{\substack{q=1 \\ m+n=q}}^{\infty}(1-$ $\left.h_{m, n} x^{m} y^{n}\right)^{-1}$, in terms of the "majorizing infinite product"

$$
1-\sum_{\substack{p=1 \\ m+n=p}}^{\infty} s^{m+n} x^{m} y^{n}=\sum_{\substack{p=1 \\ m+n=p}}^{\infty}\left(1+F_{m, n} x^{m} y^{n}\right)^{-1},
$$

where $s: \equiv \sup _{\substack{p=1 \\ m+n=p}}\left|a_{m, n}\right|^{\frac{1}{m+n}}$. The method of majorizing series is well established in the analytic theory of complex variables; see J.B. Conway [5] and S. Ghorpade and B. Limaye [28]. However, the same did not hold true for product expansions until the publication of [22]. The proof of Theorem 2.4.2 uses the fact that $\left|h_{m, n}\right| \leq$ $F_{m, n}$; see Theorem 2.4.1. The proof of this key inequality heavily relies on the algebraic representation provided by Theorem 3.2 and Equation (2.26).

The logarithm of the majorizing inverse power product plays a very important role throughout this work. Not only does it provide information regarding the domain of convergence for the IPPE2, the coefficients of the logarithmic series becomes the asymptotically dominating term. To explain the meaning of this last phrase, observe that the majorizing power product of Theorem 2.4.2 has the closed form

$$
1-\sum_{\substack{p=1 \\ m+n=p}}^{\infty} s^{m+n} x^{m} y^{n}=\frac{1-2 s(x+y)+2 s^{2} x y}{(1-s x)(1-s y)} .
$$


By setting $y=0$, Equation (2.2) provides the majorizing function for the one variable case, namely $\frac{1-2 s x}{1-s x}$. In [19], Gingold and Knopfmacher showed that the asymptotic value of $\log \frac{1-2 s x}{1-s x}=\sum_{n=0}^{\infty} d_{n} x^{n}$ coincides with the asymptotic value of its power product expansion $\prod_{n=1}^{\infty}\left(1+g_{n} x^{n}\right)$, i.e. $\lim _{n \rightarrow \infty} \frac{g_{n}}{d_{n}}=1$. In [7], the authors extended this result and showed that for fixed $M, \lim _{n \rightarrow \infty} \frac{g_{M, n}}{d_{M, n}}=1$ whenever $\log \frac{1-2 s(x+y)+2 s^{2} x y}{(1-s x)(1-s y)}=\sum_{\substack{p=1 \\ m+n=p}}^{\infty} d_{m, n} x^{m} y^{n}$. The third main result of this chapter, Theorem 2.5.1, shows that for fixed $M$, $\lim _{n \rightarrow \infty} \frac{h_{M, n}}{d_{M, n}}=1$. The proof of Theorem 2.5.1 is subtle since it requires showing that $\left|\frac{h_{M, N}}{d_{M, N}}\right|$ is bounded by a constant independent of $N$; see Lemma 2.5.1. Such a bound was not needed to prove the corresponding asymptotic result associated with the PPE2s.

This chapter is organized as follows. In Section 2, we study the expansion of a power series into an IPPE2 and provide two algebraic representations for the coefficients $h_{m, n}$ as a multivariate polynomials in $\left(a_{m, n}\right)_{\substack{m, n=0 \\ m+n=1}}^{\infty}$. The numbertheoretic representation reveals an intimate connection between the $\left(g_{m, n}\right)_{\substack{m, n=0 \\ m+n=1}}^{\infty}$ of the PPE2 and the $\left(h_{m, n}\right)_{m, n=0}^{\infty}$ of the IPPE2; see Theorem 2.2.1. In the case of one independent variable, this property reveals that $h_{m}=g_{m}$ for $m$ odd. In the case of two independent variables, this property reveals $h_{m, n}=g_{m, n}$ unless both $m$ and $n$ are even. Note that this equality is inherently implied by asymptotic results of the previous paragraph. Since $h_{m, n}=g_{m, n}$ for $m$ odd regardless of the parity $n$, we deduce that as the number of independent variables increases, so does the percentage of equality between the $h^{\prime} s$ and the $g^{\prime} s$. In Section 3, we provide another way to express recursively the coefficients $h_{m, n}$ as a multivariate polynomial of the variables $a_{m, n}$. The algebraic result of Section 3 reveals an intriguing property of these expansions. If $a_{m, n} \leq 0$, then the coefficients $h_{m, n}$ in the IPPE2 are non-positive. In Section 4, we exploit the non-positivity result of Section 3 to determine convergence conditions of the IPPE2 in terms of a majorizing power product. In Section 5, we provide an asymptotic formula for the $h_{m, n}$ associated with $1-\sum_{\substack{p=1 \\ m+n=p}}^{\infty} s^{m+n} x^{m} y^{n}$, where $s:=\sup _{\substack{p=1 \\ m+n=p}}\left|a_{m, n}\right|^{\frac{1}{m+n}}$, while in Section 6 we provide combinatorial interpretations for IPPE2 in term of partitions of $(m, n)$. Section 6 also provides a factorization for the bivariate generating function asso- 
ciated with compositions of $(m, n)$, which obey a "zero exclusivity" condition; see Proposition 2.6.1.

\subsection{Two Algebraic Formulas for the Coefficients of Inverse Power Product Expansion}

In this section and the next we study the expansion of a two variable power series into an IPPE2 and provide three algebraic representations for the coefficients $h_{m, n}$ as polynomials of the $\left(a_{m, n}\right)_{\substack{m, n=0 \\ m+n=1}}^{\infty}$.

Definition 2.2.1. Given a formal power series $1+\sum_{\substack{p=1 \\ m+n=p}}^{\infty} a_{m, n} x^{m} y^{n}$ or analytic function $f$ with $f(0,0)=1$ and a Taylor power series representation

$$
f(x, y)=1+\sum_{\substack{p=1 \\ m+n=p}}^{\infty} a_{m, n} x^{m} y^{n}
$$

we define the power product expansion of $f(x, y)$ in two independent variables, denoted by PPE2, as

$$
\begin{aligned}
f(x, y) & =\prod_{\substack{q=1 \\
m+n=q}}^{\infty}\left(1+g_{m, n} x^{m} y^{n}\right) \\
& =\left(1+g_{1,0} x^{1} y^{0}\right)\left(1+g_{0,1} x^{0} y^{1}\right)\left(1+g_{2,0} x^{2} y^{0}\right)\left(1+g_{1,1} x^{1} y^{1}\right) \ldots,
\end{aligned}
$$

and the inverse power product expansion in two independent variables, or IPPE2, of $f(x, y)$ as

$$
\begin{aligned}
f(x, y) & =\prod_{\substack{q=1 \\
m+n=q}}^{\infty}\left(1-h_{m, n} x^{m} y^{n}\right)^{-1} \\
& =\left(1-h_{1,0} x^{1} y^{0}\right)^{-1}\left(1-h_{0,1} x^{0} y^{1}\right)^{-1}\left(1-h_{2,0} x^{2} y^{0}\right)^{-1}\left(1-h_{1,1} x^{1} y^{1}\right)^{-1} \ldots
\end{aligned}
$$

where the right side of Equations (2.4) and (2.5) follow the conventions of Definition 1.2.1. 
Definition 2.3 provides a first means of obtaining an algebraic representation of $h_{m, n}$ in terms of $\left(a_{m, n}\right)$. Using Definition 1.2.3 help us describe this algebraic result.

Let

$$
f(x, y)=1+\sum_{\substack{p=1 \\ m+n=p}}^{\infty} a_{m, n} x^{m} y^{n}=\prod_{\substack{q=1 \\ m+n=q}}^{\infty}\left(1-h_{m, n} x^{m} y^{n}\right)^{-1} .
$$

By expanding the IPPE2 of (2.6) into a formal power series, coefficient comparison shows that

$$
a_{m, n}=\sum_{\substack{i_{1}+i_{2}+\cdots+i_{r}=m \\ j_{1}+j_{2}+\cdots+j_{r}=n \\(1,0) \preceq\left(i_{1}, j_{1}\right) \preceq \cdots \preceq\left(i_{r}, j_{r}\right) \preceq(m, n)}} h_{i_{1}, j_{1}} h_{i_{2}, j_{2}} \ldots h_{i_{r}, j_{r}},
$$

and that

$$
h_{m, n}=a_{m, n}-\left(\sum_{\substack{i_{1}+i_{2}+\cdots+i_{r}=m \\ j_{1}+j_{2}+\cdots+j_{r}=n,(1,0) \preceq\left(i_{1}, j_{1}\right) \preceq \cdots \preceq\left(i_{r}, j_{r}\right) \prec(m, n) \\ r \geq 2}} h_{i_{1}, j_{1}} h_{i_{2}, j_{2}} \ldots h_{i_{r}, j_{r}}\right) .
$$

To obtain the second algebraic formula which writes $h_{m, n}$ in terms of $\left(a_{m, n}\right)$, start with Equation (2.6) and take the logarithm of both sides to obtain

and obtain

$$
\log (f(x, y))=\log \left(1+\sum_{\substack{p=1 \\ m+n=p}}^{\infty} a_{m, n} x^{m} y^{n}\right)=\sum_{\substack{p=1 \\ m+n=p}}^{\infty} d_{m, n} x^{m} y^{n},
$$

$$
\begin{aligned}
\log (f(x, y))=\log \prod_{\substack{q=1 \\
m+n=q}}^{\infty}\left(1-h_{m, n} x^{m} y^{n}\right)^{-1} & :=-\sum_{\substack{q=1 \\
m+n=q}}^{\infty} \log \left(1-h_{m, n} x^{m} y^{n}\right) \\
& :=\sum_{\substack{q=1 \\
m+n=q}}^{\infty} \sum_{l=1}^{\infty} \frac{\left(h_{m, n} x^{m} y^{n}\right)^{l}}{l} .
\end{aligned}
$$

Compare the coefficient of $x^{M} y^{N}$ on both sides of Equation (2.9) to obtain the formulas

$$
d_{M, N}=\frac{1}{M} \sum_{\substack{m \mid M \\ n=\frac{N m}{M}}} m\left(h_{m, n}\right)^{\frac{M}{m}}, \quad M \neq 0
$$




$$
d_{M, N}=\frac{1}{N} \sum_{\substack{n \mid N \\ m=\frac{M n}{N}}} n\left(h_{m, n}\right)^{\frac{N}{n}}, \quad N \neq 0
$$

Take Equation (2.10) and set $m=M$ to obtain

$$
h_{M, N}=d_{M, N}-\frac{1}{M} \sum_{\substack{m \mid M \\ m \neq M}} m\left(h_{m, \frac{N m}{m}}\right)^{\frac{M}{m}}
$$

Similarly, take Equation (2.11) and set $n=N$ to obtain

$$
h_{M, N}=d_{M, N}-\frac{1}{N} \sum_{\substack{n \mid N \\ n \neq N}} n\left(h_{\frac{M n}{N}, n}\right)^{\frac{N}{n}}
$$

If $\operatorname{gcd}(M, N)=1$, then we readily deduce that

$$
h_{M, N}=d_{M, N}
$$

A similar derivation to the one above shows that

$$
g_{M, N}=d_{M, N}+\frac{1}{M} \sum_{\substack{m \mid M \\ m \neq M}} m\left(-g_{m, \frac{N m}{M}}\right)^{\frac{M}{m}}
$$

and that

$$
g_{M, N}=d_{M, N}, \quad \text { where } \operatorname{gcd}(M, N)=1 .
$$

Equations (2.12) and (2.15) enable us to prove a theorem which indicates the relationship between the coefficients of PPE2 and its associated IPPE2.

Theorem 2.2.1. Let $f(x, y)=1+\sum_{\substack{p=1 \\ m+n=p}}^{\infty} a_{m, n} x^{m} y^{n}$. Suppose $f(x, y)$ has a PPE2 of the form $f(x, y)=\prod_{\substack{q=1 \\ m+n=q}}^{\infty}\left(1+g_{m, n} x^{m} y^{n}\right)$ and an IPPE2 of the form $f(x, y)=\prod_{\substack{q=1 \\ m+n=q}}^{\infty}\left(1-h_{m, n} x^{m} y^{n}\right)^{-1}$. Then $g_{m, n}=h_{m, n}$ if $m=2 \ell+1$ or $n=2 \ell+1$ for $\ell \in \mathbb{N}$ 
Proof. We prove the statement that if $m$ is odd, then $g_{m, n}=h_{m, n}$ by induction on $\ell$. The other statement involving $n$ is similar. If $\ell=0$ and $n \in \mathbb{N}_{0}$, Equation (2.14) implies that $h_{1, n}=d_{1, n}$, while Equation (2.16) implies that $g_{1, n}=d_{1, n}$. Hence, $g_{1, n}=h_{1, n}$. Now assume $g_{2 \ell+1, n}=h_{2 \ell+1, n}$ for all $0 \leq \ell \leq L$ and $0 \leq n \leq N$. Take Equation (2.15) and with $\ell=L+1$ and note that

$$
\begin{aligned}
g_{2 L+3, N} & =d_{2 L+3, N}+\frac{1}{2 L+3} \sum_{\substack{m \mid 2 L+3 \\
m \neq 2 L+3 \\
n=\frac{N m}{2 L+3}}} m\left(-g_{m, n}\right)^{\frac{2 L+3}{m}} \\
& =d_{2 L+3, N}-\frac{1}{2 L+3} \sum_{\substack{m \mid 2 L+3 \\
m \neq 2 L+3 \\
n=\frac{N m}{2 L+3}}} m\left(g_{m, n}\right)^{\frac{2 L+3}{m}} \\
& =d_{2 L+3, N}-\frac{1}{2 L+3} \sum_{\substack{m \mid 2 L+3 \\
m \neq 2 L+3 \\
n=\frac{N m}{2 L+3}}} m\left(h_{m, n}\right)^{\frac{2 L+3}{m}} \\
& =h_{2 L+3, N}, \quad
\end{aligned}
$$

where the second to last equality used the induction hypothesis and the last equality used Equation (2.12).

\subsection{Structure Property of the Coefficients of an In- verse Power Product Expansion}

There is still another way to express recursively the coefficients $h_{m, n}$ as a multivariate polynomial of the variables $a_{m, n}$. Start by rewriting Equation (2.6) as

$$
f(x, y)=1+\sum_{\substack{p=1 \\ m+n=p}}^{\infty} B_{(1,0),(m, n)} x^{m} y^{n}=\prod_{\substack{q=1 \\ m+n=q}}^{\infty}\left(1-h_{m, n} x^{m} y^{n}\right)^{-1},
$$

where $B_{(1,0),(m, n)}=a_{m, n}$ for all $(m, n) \in \mathbb{N}_{0} \times \mathbb{N}_{0} \backslash\{(0,0)\}$. Using the well ordering 1.2.1 to factor $\prod_{\substack{q=1 \\ m+n=q}}^{\infty}\left(1-h_{m, n} x^{m} y^{n}\right)^{-1}$, we get the following recursive system 
of equations. First,

$$
\begin{aligned}
f(x, y) & =1+\sum_{\substack{p=1 \\
m+n=p}}^{\infty} B_{(1,0),(m, n)} x^{m} y^{n} \\
& =\left(1-h_{1,0} x\right)^{-1} \prod_{\substack{q=1 \\
m+n=q \\
(m, n) \succeq(0,1)}}^{\infty}\left(1-h_{m, n} x^{m} y^{n}\right)^{-1} \\
& =\left(1-h_{1,0} x\right)^{-1}\left[1+\sum_{\substack{p=1 \\
m+n=p \\
(m, n) \succeq(0,1)}}^{\infty} B_{(0,1),(m, n)} x^{m} y^{n}\right],
\end{aligned}
$$

secondly,

$$
\begin{aligned}
& 1+\sum_{\substack{p=1 \\
m+n=p \\
(m, n) \succeq(0,1)}}^{\infty} B_{(0,1),(m, n)} x^{m} y^{n}=\left(1-h_{0,1} y\right)^{-1} \prod_{\substack{q=2 \\
m+n=q \\
(m, n) \succeq(2,0)}}^{\infty}\left(1-h_{m, n} x^{m} y^{n}\right)^{-1} \\
& =\left(1-h_{0,1} y\right)^{-1}\left[1+\sum_{\substack{q=2 \\
m+n=q \\
(m, n) \succeq(2,0)}}^{\infty} B_{(2,0),(m, n)^{2}} x^{m} y^{n}\right]
\end{aligned}
$$

and thirdly,

$$
\begin{aligned}
1+ & \sum_{\substack{q=2 \\
m+n=q \\
(m, n) \succeq(2,0)}}^{\infty} B_{(2,0),(m, n)} x^{m} y^{n}=\left(1-h_{2,0} x^{2}\right)^{-1} \prod_{\substack{q=2 \\
m+n=q,(m, n) \succeq(1,1)}}^{\infty}\left(1-h_{m, n} x^{m} y^{n}\right)^{-1} \\
= & \left(1-h_{2,0} x^{2}\right)^{-1}\left[1+\sum_{\substack{p=2 \\
m+n=p,(m, n) \succeq(1,1)}}^{\infty} B_{(1,1),(m, n)^{2}} x^{m} y^{n}\right] .
\end{aligned}
$$

Continue this process to inductively define

$$
\begin{gathered}
1+\sum_{\substack{p=\ell \\
m+n=p \\
(m, n) \succeq\left(i_{k}, j_{k}\right)}}^{\infty} B_{\left(i_{k}, j_{k}\right),(m, n)} x^{m} y^{n}=\left(1-h_{i_{k}, j_{k}} x^{i_{k}} y^{j_{k}}\right)^{-1} \prod_{\substack{q=\ell \\
m+n=q \\
(m, n) \succeq\left(i_{k+1}, j_{k+1}\right)}}^{\infty}\left(1-h_{m, n} x^{m} y^{n}\right)^{-1} \\
=\left(1-h_{i_{k}, j_{k}} x^{i_{k}} y^{j_{k}}\right)^{-1}\left[1+\sum_{\substack{p=\ell \\
m+n=p \\
(m, n) \succeq\left(i_{k+1}, j_{k+1}\right)}}^{\infty} B_{\left(i_{k+1}, j_{k+1}\right),(m, n)} x^{m} y^{n}\right] .
\end{gathered}
$$


Expanding the final equality of Equation (2.17) implies that

$$
\begin{gathered}
1+\sum_{\substack{p=\ell \\
m+n=p}}^{\infty} B_{\left(i_{k}, j_{k}\right),(m, n)} x^{m} y^{n}=\left(1-h_{i_{k}, j_{k}} x^{i_{k}} y^{i_{k}}\right)^{-1}\left[1+\sum_{\substack{p=\ell \\
m+n=p \\
(m, n) \succeq\left(i_{k}, j_{k}\right)}}^{\infty} B_{\left(i_{k+1}, j_{k+1}\right),(m, n)} x^{m} y^{n}\right] \\
=\left[1+\sum_{\alpha=1}^{\infty} h_{\left.i_{k}, j_{k}, j_{k+1}\right)}^{\alpha}\left(x^{i_{k}} y^{i_{k}}\right)^{\alpha}\right]\left[1+\sum_{\substack{p=\ell \\
m+n=p \\
(m, n) \succeq\left(i_{k+1}, j_{k+1}\right)}}^{\infty} B_{\left(i_{k+1}, j_{k+1}\right),(m, n)} x^{m} y^{n}\right] .
\end{gathered}
$$

By comparing the coefficient of $x^{M} y^{N}$ in both sides of Equation (2.18), we discover that

$$
B_{\left(i_{k+1}, j_{k+1}\right),(M, N)}=B_{\left(i_{k}, j_{k}\right),(M, N)}-\sum_{\alpha=1}^{\left\lfloor\frac{M+N}{i_{k}+j_{k}}\right\rfloor} h_{i_{k}, j_{k}}^{\alpha} B_{\left(i_{k+1}, j_{k+1}\right),\left(M-\alpha i_{k+1}, N-\alpha j_{k+1}\right)} .
$$

When $(M, N)=\left(i_{k}, j_{k}\right)$, since $B_{\left(i_{k+1}, j_{k+1}\right),\left(i_{k}, j_{k}\right)}=0$, the above implies that

$$
h_{i_{k}, j_{k}}=B_{\left(i_{k}, j_{k}\right),\left(i_{k}, j_{k}\right)} \text {. }
$$

Equation (2.19) shows the relationship between $h_{i_{k}, j_{k}}$ and $B_{\left(i_{k}, j_{k}\right),\left(i_{k}, j_{k}\right)}$. We use this relationship to rewrite Equation (2.17) as

$$
\begin{aligned}
& 1+\sum_{\substack{p=\ell \\
m+n=p}}^{\infty} B_{\left(i_{k+1}, j_{k+1}\right),(m, n)} x^{m} y^{n}=\left(1-h_{i_{k}, j_{k}} x^{i_{k}} y^{i_{k}}\right)\left[1+\sum_{\substack{p=\ell \\
m+n=p,(m, n) \succeq\left(i_{k}, j_{k}\right)}}^{\infty} B_{\left(i_{k}, j_{k}\right),(m, n)} x^{m} y^{n}\right] \\
& =\left[1-B_{\left(i_{k}, j_{k}\right),\left(i_{k}, j_{k}\right)} x^{i_{k}} y^{i_{k}}\right]\left[1+\sum_{\substack{p=\ell \\
m+n=p \\
(m, n) \succeq\left(i_{k}, j_{k}\right)}}^{\infty} B_{\left(i_{k}, j_{k}\right),(m, n)} x^{m} y^{n}\right] .
\end{aligned}
$$

Equating the coefficient of $x^{s} y^{t}$ on both sides of Equation (2.20), we get

$$
B_{\left(i_{k+1}, j_{k+1}\right),(s, t)}=\sum_{\substack{\alpha \in\{0,1\} \\ \alpha i_{k}+m=s \\ \alpha j_{k}+n=t}}(-1)^{\alpha} B_{\left(i_{k}, j_{k}\right),\left(i_{k}, j_{k}\right)}^{\alpha} B_{\left(i_{k}, j_{k}\right),\left(s-\alpha i_{k}, t-\alpha j_{k}\right)} .
$$

Equation (2.21) will be the main tool for proving the following result. 
Theorem 2.3.1. Let $\left(i_{k}, j_{k}\right) \in \mathbb{N}_{0} \times \mathbb{N}_{0} \backslash\{(0,0)\}$. Define $B_{\left(i_{k}, j_{k}\right),(0,0)}=1$ and $B_{\left(i_{k}, j_{k}\right),(m, n)}=0$ for $(1,0) \preceq(m, n) \preceq\left(i_{k-1}, j_{k-1}\right)$. Assume that $B_{\left(i_{k}, j_{k}\right),(m, n)} \leq 0$ for all $\left(i_{k}, j_{k}\right) \preceq(m, n)$. Then $B_{\left(i_{k+1}, j_{k+1}\right),(s, t)} \leq 0$ whenever $\left(i_{k+1}, j_{k+1}\right) \preceq(s, t)$.

Proof. Equation (2.21) is equivalent to

$$
B_{\left(i_{k+1}, j_{k+1}\right),(s, t)}=B_{\left(i_{k}, j_{k}\right),(s, t)}-B_{\left(i_{k}, j_{k}\right),\left(i_{k}, j_{k}\right)} B_{\left(i_{k}, j_{k}\right),\left(s-i_{k}, t-j_{k}\right)} .
$$

Rewrite Equation (2.22) as $B_{\left(i_{k+1}, j_{k+1}\right),(s, t)}=\beta+\gamma$, where

$$
\begin{aligned}
\beta & :=B_{\left(i_{k}, j_{k}\right),(s, t)}, \\
\gamma & :=-B_{\left(i_{k}, j_{k}\right),\left(i_{k}, j_{k}\right)} B_{\left(i_{k}, j_{k}\right),\left(s-i_{k}, t-j_{k}\right)} .
\end{aligned}
$$

By the hypothesis, since $B_{\left(i_{k}, j_{k}\right),(s, t)}$ is either zero or negative, then $\beta$ is nonpositive.

It remains to show that $\gamma$ is also non-positive. By the hypothesis, it is a product of two non-positive numbers. Thus $-B_{\left(i_{k}, j_{k}\right),\left(i_{k}, j_{k}\right)} B_{\left(i_{k}, j_{k}\right),\left(s-i_{k}, t-j_{k}\right)}$ is either zero or negative.

Before stating the main theorem of this section, we introduce some further notation.

Definition 2.3.1. The symbol $\phi=\left(\left(i_{1}, j_{1}\right),\left(i_{2}, j_{2}\right), \ldots,\left(i_{n}, j_{n}\right)\right)$ stands for a vector with $n$ components, where $n \in \mathbb{N}$ and $i_{1}, i_{2}, \ldots, i_{n}, j_{1}, j_{2}, \ldots, j_{n} \in \mathbb{N}_{0}$. Let $\tau=\tau(\phi)$ be the length of $\phi$, i.e. $\tau=n$. Let $|\phi|$ denote the ordered pair $|\phi|:=\left(\sum_{d=1}^{n} i_{d}, \sum_{d=1}^{n} j_{d}\right)$. We define $B_{\left(i_{k}, j_{k}\right), \phi}:=B_{\left(i_{k}, j_{k}\right),\left(i_{1}, j_{1}\right)} B_{\left(i_{k}, j_{k}\right),\left(i_{2}, j_{2}\right)} \ldots B_{\left(i_{k}, j_{k}\right),\left(i_{n}, j_{n}\right)}$.

Theorem 2.3.2. (Structure Property) Let $\left(i_{k}, j_{k}\right) \in \mathbb{N}_{0} \times \mathbb{N}_{0} \backslash\{(0,0)\}$. Then

$$
\begin{aligned}
B_{\left(i_{k+1}, j_{k+1}\right),(s, t)} & =\sum_{\eta}(-1)^{\tau(\phi(\eta))-1} B_{\left(i_{k}, j_{k}\right), \phi(\eta)} \\
& =\sum_{\eta}(-1)^{\tau(\phi(\eta))+1} B_{\left(i_{k}, j_{k}\right), \phi(\eta)},
\end{aligned}
$$

where the sum is over all $\phi(\eta)=\left(\left(i_{1}, j_{1}\right),\left(i_{2}, j_{2}\right), \ldots,\left(i_{\tau}, j_{\tau}\right)\right)$ such that $|\phi(\eta)|=$ $(s, t)$ and at most one component $\left(l_{\theta}, l_{\theta}^{\prime}\right) \neq\left(i_{k}, j_{k}\right)$, with $1 \leq \theta \leq \tau$, and $B_{\left(i_{k}, j_{k}\right), \phi(\eta)}:=B_{\left(i_{k}, j_{k}\right),\left(i_{1}, j_{1}\right)} B_{\left(i_{k}, j_{k}\right),\left(i_{2}, j_{2}\right)} \ldots B_{\left(i_{k}, j_{k}\right),\left(i_{\tau}, j_{\tau}\right)}$. Note that $\eta$ represents the enumeration of the vectors that obey the two properties described in the preceding sentence. If $B_{\left(i_{k}, j_{k}\right),(s, t)} \leq 0$ for all $\left(i_{k}, j_{k}\right) \preceq(s, t)$, then Equation (2.23) is equivalent to

$$
B_{\left(i_{k+1}, j_{k+1}\right),(s, t)}=-\sum_{\eta}\left|B_{\left(i_{k}, j_{k}\right),\left(i_{1}, j_{1}\right)}\right| \ldots\left|B_{\left(i_{k}, j_{k}\right),\left(i_{\tau}, j_{\tau}\right)}\right| .
$$


Proof. We obtain the desired result by representing $B_{\left(i_{k}, j_{k}\right),\left(i_{k}, j_{k}\right)}^{\alpha} B_{\left(i_{k}, j_{k}\right),(m, n)}$ in Equation (2.21) as $B_{\left(i_{k}, j_{k}\right), \phi(\eta)}$. Note that $(-1)^{\alpha}=(-1)^{\tau(\phi(\eta))-1}$.

Let us see what will happen when we recursively apply Equation (2.24). In order to efficiently record the results, let $\phi=\left(\left(i_{1}, j_{1}\right),\left(i_{2}, j_{2}\right), \ldots,\left(i_{n}, j_{n}\right)\right)$ denote a vector with $n$ components, each of them an ordered pair, where $n \in \mathbb{N}$, and $i_{1}, i_{2}, \ldots, i_{n}, j_{1}, j_{2}, \ldots, j_{n} \in \mathbb{N}_{0}$. Then $a_{\phi(\eta)}$ denotes the expression $a_{i_{1}, j_{1}} a_{i_{2}, j_{2}} \ldots a_{i_{n}, j_{n}}$. After $L$ iterations, and assuming $B_{\left(i_{k}, j_{k}\right),(s, t)} \leq 0$, whenever $\left(i_{k}, j_{k}\right) \preceq(s, t)$, we obtain

$$
B_{\left(i_{k+1}, j_{k+1}\right),(s, t)}=\sum_{\eta}(-1)^{\tau(\phi(\eta))-1} a_{\phi(\eta)}=-\sum_{\eta}\left|a_{i_{1}, j_{1}}\right|\left|a_{i_{2}, j_{2}}\right| \cdots\left|a_{i_{\tau}, j_{\tau}}\right|,
$$

where the sum is over all $\phi(\eta)=\left(\left(i_{1}, j_{1}\right),\left(i_{2}, j_{2}\right), \ldots,\left(i_{\tau}, j_{\tau}\right)\right)$ such that $|\phi(\eta)|=$ $(s, t)$.

$$
\begin{aligned}
& \text { If }(s, t)=\left(i_{k+1}, j_{k+1}\right), \text { Equation (2.25) becomes } \\
& \qquad \begin{aligned}
B_{\left(i_{k+1}, j_{k+1}\right),\left(i_{k+1}, j_{k+1}\right)}=h_{i_{k+1}, j_{k+1}} & =\sum_{\eta}(-1)^{\tau(\phi(\eta))-1} a_{\phi(\eta)}, \\
& =-\sum_{\eta}\left|a_{i_{1}, j_{1}}\right|\left|a_{i_{2}, j_{2}}\right| \ldots\left|a_{i_{\tau}, j_{\tau}}\right|,
\end{aligned}
\end{aligned}
$$

where the sum is over all $\phi(\eta)=\left(\left(i_{1}, j_{1}\right),\left(i_{2}, j_{2}\right), \cdots,\left(i_{\tau}, j_{\tau}\right)\right)$ such that $|\phi(\eta)|=$ $\left(i_{k+1}, j_{k+1}\right)$.

The following example illustrates applications of the structure property:

$$
\begin{array}{rlr}
h_{2,1}= & B_{(2,1),(2,1)} & \\
= & B_{(3,0),(2,1)} & \\
= & B_{(0,2),(2,1)} \text { By using Equation(2.19) } \\
= & B_{(1,1),(2,1)} & \\
= & B_{(2,0),(2,1)} & \\
= & B_{(0,1),(2,1)}-B_{(0,1),(0,1)} B_{(0,1),(2,0)} & \\
= & B_{(1,0),(2,1)}-B_{(1,0),(1,0)} B_{(1,0),(1,1)}-B_{(1,0),(0,1)}\left[B_{(1,0),(2,0)}-\right. \\
& \left.B_{(1,0),(1,0)} B_{(1,0),(1,0)}\right] & \\
= & a_{2,1}-a_{1,0} a_{1,1}-a_{0,1} a_{2,0}+a_{1,0}^{2} a_{0,1} . &
\end{array}
$$

Below we explicitly list $h_{m, n}$ for $1 \leq m+n \leq 4$. 


$$
\begin{aligned}
h_{1,0}= & (-1)^{0} a_{1,0} \\
h_{0,1}= & (-1)^{0} a_{0,1} \\
h_{2,0}= & (-1)^{0} a_{2,0}+(-1)^{1} a_{1,0}^{2} \\
h_{1,1}= & (-1)^{0} a_{1,1}+(-1)^{1} a_{1,0} a_{0,1} \\
h_{0,2}= & (-1)^{0} a_{0,2}+(-1)^{1} a_{0,1}^{2} \\
h_{3,0}= & (-1)^{0} a_{3,0}+(-1)^{1} a_{2,0} a_{1,0} \\
h_{2,1}= & (-1)^{0} a_{2,1}+(-1)^{1} a_{0,1} a_{2,0}+(-1)^{1} a_{1,0} a_{1,1}+(-1)^{2} a_{1,0}^{2} a_{0,1} \\
h_{1,2}= & (-1)^{0} a_{1,2}+(-1)^{1} a_{1,0} a_{0,2}+(-1)^{1} a_{0,1} a_{1,1}+(-1)^{2} a_{0,1}^{2} a_{1,0} \\
h_{0,3}= & (-1)^{0} a_{0,3}+(-1)^{1} a_{0,2} a_{0,1} \\
h_{4,0}= & (-1)^{0} a_{4,0}+(-1)^{1} a_{1,0} a_{3,0}+(-1)^{1} a_{2,0}^{2}+(-1)^{2} 2 a_{2,0} a_{1,0}^{2}+(-1)^{3} a_{1,0}^{4} \\
h_{3,1}= & (-1)^{0} a_{3,1}+(-1)^{3} a_{1,0}^{3} a_{0,1}+(-1)^{2} a_{1,0}^{2} a_{1,1}+(-1)^{1} a_{0,1} a_{3,0}+ \\
& (-1)^{1} a_{1,1} a_{2,0}+(-1)^{1} a_{1,0} a_{2,1}+(-1)^{2} 2 a_{0,1} a_{1,0} a_{2,0} \\
h_{2,2}= & (-1)^{0} a_{2,2}+(-1)^{1} a_{2,0} a_{0,2}+(-1)^{3} 2 a_{1,0}^{2} a_{0,1}^{2}+(-1)^{1} a_{0,1} a_{2,1}+ \\
& (-1)^{2} a_{0,1}^{2} a_{2,0}+(-1)^{2} a_{1,0}^{2} a_{0,2}+(-1)^{2} 2 a_{1,0} a_{0,1} a_{1,1}+(-1)^{1} a_{1,0} a_{1,2}+ \\
& (-1)^{2} a_{1,0} a_{1,0} a_{0,2} \\
h_{1,3}= & (-1)^{0} a_{1,3}+(-1)^{3} a_{0,1}^{3} a_{1,0}+(-1)^{2} a_{0,1}^{2} a_{1,1}+(-1)^{1} a_{1,0} a_{0,3}+(-1)^{1} a_{1,1} a_{0,2} \\
& +(-1)^{1} a_{0,1} a_{1,2}+(-1)^{2} 2 a_{0,1} a_{1,0} a_{0,2} \\
h_{0,4}= & (-1)^{0} a_{0,4}+(-1)^{1} a_{0,1} a_{0,3}+(-1)^{1} a_{0,2}^{2}+(-1)^{2} 2 a_{0,2} a_{0,1}^{2}+(-1)^{3} a_{0,1}^{4} . \\
&
\end{aligned}
$$

\subsection{Convergence Criteria For Inverse Power Prod- uct}

The major results of this section concerning the convergence domain of the IPPE2 in Equation (2.1).

Theorem 2.4.1. Let $f(x, y)=1+\sum_{\substack{p=1 \\ m+n=p}}^{\infty} a_{m, n} x^{m} y^{n}$. Then $f(x, y)$ is represented by the IPPE2

$$
f(x, y)=1+\sum_{\substack{p=1 \\ m+n=p}}^{\infty} a_{m, n} x^{m} y^{n}=\prod_{\substack{q=1 \\ m+n=q}}^{\infty}\left(1-h_{m, n} x^{m} y^{n}\right)^{-1}
$$


Consider the following auxiliary functions

$$
\begin{aligned}
& C(x, y)=1-\sum_{\substack{p=1 \\
m+n=p}}^{\infty}\left|a_{m, n}\right| x^{m} y^{n}=\prod_{\substack{q=1 \\
m+n=q}}^{\infty}\left(1+\hat{H}_{m, n} x^{m} y^{n}\right)^{-1}, \\
& D(x, y)=1-\sum_{\substack{p=1 \\
m+n=p}}^{\infty} A_{m, n} x^{m} y^{n}=\prod_{\substack{q=1 \\
m+n=q}}^{\infty}\left(1+F_{m, n} x^{m} y^{n}\right)^{-1}
\end{aligned}
$$

Assume $\left|a_{m, n}\right| \leq A_{m, n}$ for all $(m, n) \in \mathbb{N}_{0} \times \mathbb{N}_{0} \backslash\{(0,0)\}$. Then $\left|h_{m, n}\right| \leq \hat{H}_{m, n} \leq F_{m, n}$ for all $(m, n) \in \mathbb{N}_{0} \times \mathbb{N}_{0} \backslash\{(0,0)\}$.

Proof. By Equation (2.26) we have

$$
h_{m, n}=\sum_{\substack{\eta \\|\phi(\eta)|=(m, n)}}(-1)^{\tau(\phi(\eta))-1} a_{\phi(\eta)}=\sum_{\substack{\eta \\|\phi(\eta)|=(m, n)}}(-1)^{\tau(\phi(\eta))-1} a_{i_{1}, j_{1}} a_{i_{2}, j_{2}} \ldots a_{i_{\tau}, j_{\tau}} .
$$

Equation (2.30) implies that

$$
\left|h_{m, n}\right|=\left|\sum_{\substack{\eta \\|\phi(\eta)|=(m, n)}}(-1)^{\tau(\phi(\eta))-1} a_{i_{1}, j_{1}} a_{i_{2}, j_{2}} \ldots a_{i_{\tau}, j_{\tau}}\right| \leq \sum_{\substack{\eta \\|\phi(\eta, \eta)|=(m, n)}}\left|a_{i_{1}, j_{1}}\right|\left|a_{i_{2}, j_{2}}\right| \ldots\left|a_{i_{\tau}, j_{\tau}}\right| .
$$

Similarly, when we apply Equation (2.26) to Equation (2.28), we obtain

$$
\begin{aligned}
0 \leq \hat{H}_{m, n} & =\sum_{\substack{\eta \\
|\phi(\eta)|=(m, n)}}(-1)^{\tau(\phi(\eta))}\left(-\left|a_{i_{1}, j_{1}^{\prime}}\right|\right)\left(-\left|a_{i_{2}, j_{2}}\right|\right) \ldots\left(-\left|a_{i_{\tau}, j_{\tau}}\right|\right) \\
& =\sum_{\substack{\eta \\
|\phi(\eta)|=(m, n)}}(-1)^{\tau(2 \phi(\eta))}\left|a_{i_{1}, j_{1}}\right|\left|a_{i_{2}, j_{2}}\right| \ldots\left|a_{i_{\tau}, j_{\tau}}\right| \\
& =\sum_{\substack{\eta \\
|\phi(\eta)|=(m, n)}}\left|a_{i_{1}, j_{1}}\right|\left|a_{i_{2}, j_{2}}\right| \ldots\left|a_{i_{\tau}, j_{\tau}}\right| .
\end{aligned}
$$

Equations (2.31) and (2.32) imply that $\left|h_{m, n}\right| \leq \hat{H}_{m, n}$. Also, due to the inequality $\left|a_{m, n}\right| \leq A_{m, n}$, we have

$$
0 \leq \hat{H}_{m, n}=\sum_{\substack{\eta \\|\phi(\eta)|=(m, n)}}\left|a_{i_{1}, j_{1}}\right|\left|a_{i_{2}, j_{2}}\right| \ldots\left|a_{i_{\tau}, j_{\tau}}\right| \leq \sum_{\substack{\eta \\|\phi(\eta)|=(m, n)}} A_{i_{1}, j_{1}} A_{i_{2}, j_{2}} \ldots A_{i_{\tau}, j_{\tau}}=F_{m, n} .
$$


We now consider the special case for $D(x, y)$, namely

$$
D(x, y)=1-\sum_{\substack{p=1 \\ m+n=p}}^{\infty} s^{m+n} x^{m} y^{n}=\prod_{\substack{q=1 \\ m+n=q}}^{\infty}\left(1+F_{m, n} x^{m} y^{n}\right)^{-1}
$$

where $s: \equiv \sup _{\substack{p=1 \\ m+n=p}}\left|a_{m, n}\right|^{\frac{1}{m+n}}$. Our objective is to find a domain of definition such that the IPPE2 of Equation (2.33) is absolutely convergent. Define

$$
\log \left(1+F_{m, n} x^{m} y^{n}\right)^{-1}:=-\log \left(1+F_{m, n} x^{m} y^{n}\right):=\sum_{\ell=1}^{\infty} \frac{(-1)^{\ell}\left(F_{m, n} x^{m} y^{n}\right)^{\ell}}{\ell} .
$$

Next, define (via Definition 1.2.1)

$$
-\sum_{\substack{p=1 \\ m+n=p}}^{\infty} \log \left(1+F_{m, n} x^{m} y^{n}\right):=\sum_{\substack{p=1 \\ m+n=p}}^{\infty} \sum_{\ell=1}^{\infty} \frac{(-1)^{\ell}\left(F_{m, n} x^{m} y^{n}\right)^{\ell}}{\ell}
$$

Definition (2.34) tells us that absolute convergence of the double series implies that both $\sum_{\substack{p=1 \\ m+n=p}}^{\infty} \log \left(1+F_{m, n} x^{m} y^{n}\right)$ and $\log \left(1+F_{m, n} x^{m} y^{n}\right)$ are absolutely convergent. Moreover, since

$$
\begin{aligned}
\exp \left(-\sum_{\substack{p=1 \\
m+n=p}}^{\infty} \log \left(1+F_{m, n} x^{m} y^{n}\right)\right) & =\exp \left(\sum_{\substack{p=1 \\
m+n=p}}^{\infty} \log \left(1+F_{m, n} x^{m} y^{n}\right)^{-1}\right) \\
& =\prod_{\substack{q=1 \\
m+n=q}}^{\infty}\left(1+F_{m, n} x^{m} y^{n}\right)^{-1},
\end{aligned}
$$

the absolute convergence of the double series of Equation (2.34) implies the absolute convergence of $\prod_{\substack{q=1 \\ m+n=q}}^{\infty}\left(1+F_{m, n} x^{m} y^{n}\right)^{-1}$ as well. Therefore, in order to determine where $\prod_{\substack{q=1 \\ m+n=q}}^{\infty}\left(1+F_{m, n} x^{m} y^{n}\right)^{-1}$ will be absolutely convergent, it suffices to consider the absolutely convergence of $\sum_{\substack{p=1 \\ m+n=p}}^{\infty} \log \left(1+F_{m, n} x^{m} y^{n}\right)^{-1}$. Observe that this argument is a two variable extension of Proposition 5.2 and Definition 5.5 of [5]. 
Taking the logarithm of both sides of Equation (2.33) gives

$$
\begin{aligned}
\log \left(1-\sum_{\substack{p=1 \\
m+n=p}}^{\infty} s^{m+n} x^{m} y^{n}\right) & =\log \prod_{\substack{q=1 \\
m+n=q}}^{\infty}\left(1+F_{m, n} x^{m} y^{n}\right)^{-1} \\
& =-\sum_{\substack{q=1 \\
m+n=q}}^{\infty} \log \left(1+F_{m, n} x^{m} y^{n}\right)
\end{aligned}
$$

Observe that

$$
\begin{aligned}
1-\sum_{\substack{p=1 \\
m+n=p}}^{\infty} s^{m+n} x^{m} y^{n} & =1-\sum_{\substack{p=1 \\
m+n=p}}^{\infty}(s x)^{m}(s y)^{n}=2-\sum_{m=0}^{\infty}(s x)^{m} \sum_{n=0}^{\infty}(s y)^{n} \\
& =2-\left(\frac{1}{1-s x}\right)\left(\frac{1}{1-s y}\right)=\frac{1-2 s(x+y)+2 s^{2} x y}{(1-s x)(1-s y)} .
\end{aligned}
$$

Hence,

$$
\begin{aligned}
\log & \left(\frac{1-2 s(x+y)+2 s^{2} x y}{(1-s x)(1-s y)}\right) \\
& =\log \left(1-\left[2 s(x+y)-2 s^{2} x y\right]\right)-\log (1-s x)-\log (1-s y) \\
& =\sum_{\ell=1}^{\infty} \frac{(s x)^{\ell}}{\ell}+\sum_{\ell=1}^{\infty} \frac{(s y)^{\ell}}{\ell}-\sum_{\ell=1}^{\infty} \frac{\left[2 s(x+y)-2 s^{2} x y\right]^{\ell}}{\ell} .
\end{aligned}
$$

The three series in (2.36) are absolutely convergent for $|x|<\frac{1}{s},|y|<\frac{1}{s}$, and for $\left|2 s(x+y)-2 s^{2} x y\right|<1$, respectively. By the triangle inequality, we have

$$
\left|2 s(x+y)-2 s^{2} x y\right| \leq 2 s(|x|+|y|)+2 s^{2}|x||y| .
$$

If we require $2 s(|x|+|y|)+2 s^{2}|x||y|<1$, since $2 s|x|<2 s(|x|+|y|)+2 s^{2}|x||y|<1$, we find that $|x|<\frac{1}{2 s}$. Similarly, $|y|<\frac{1}{2 s}$.

Therefore, the estimate of a convergence domain of (2.36) is given by

$$
\mathbb{D}=\left\{(x, y) \in \mathbb{C}^{2}: 2 s(|x|+|y|)+2 s^{2}|x||y|<1\right\} .
$$

See Figure 2.1. 


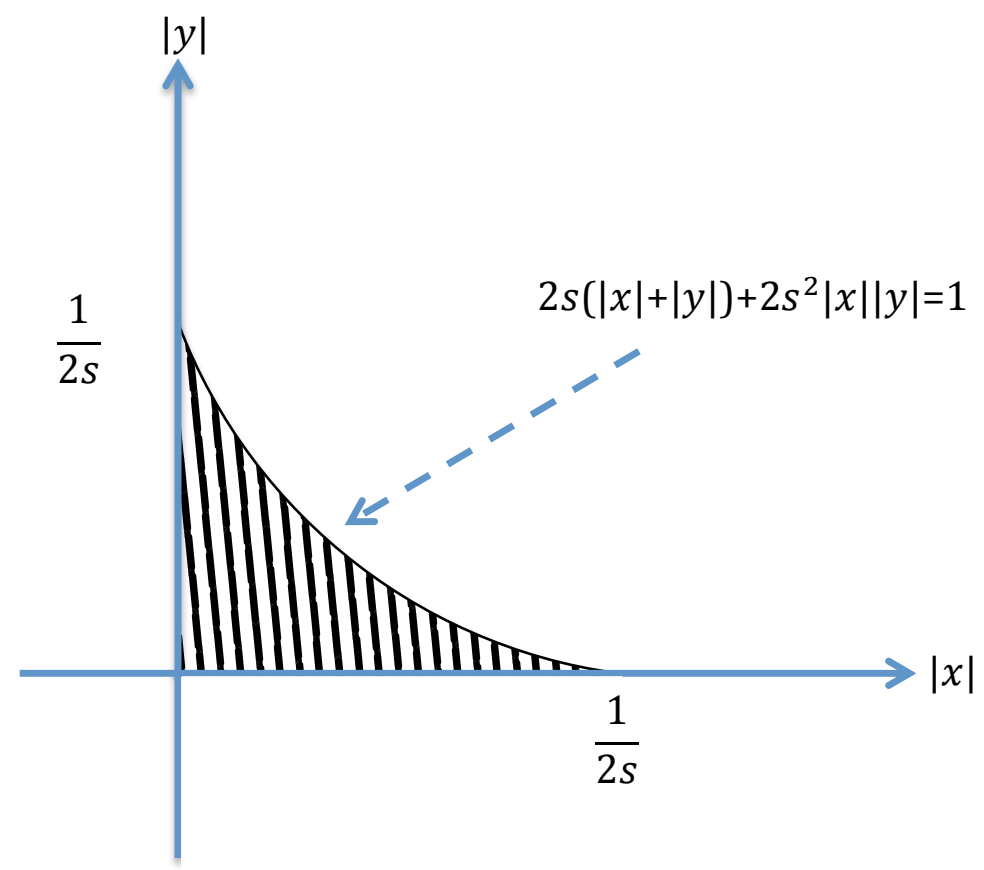

Figure 2.1: A domain of absolute convergence of Equations (2.34) and (2.36).

Relation (2.37) makes it possible to also obtain a domain of absolute convergence in terms of polydiscs. Let $|x|<\rho$ and $|y|<\rho$. Inequality (2.37) implies that the IPPE2 will be absolutely convergent if

$$
2 s(2 \rho)+2 s^{2} \rho^{2}=2\left[(s \rho+1)^{2}-1\right]<1,
$$

or equivalently if $\rho<s^{-1}\left[\sqrt{\frac{3}{2}}-1\right]$. The inequalities for $\rho$ obtained from the defining quadratic equation of $\mathbb{D}$, namely

$$
|x|<s^{-1}\left[\sqrt{\frac{3}{2}}-1\right], \quad|y|<s^{-1}\left[\sqrt{\frac{3}{2}}-1\right],
$$

are sharp in the sense that if

$$
x=-s^{-1}\left[\sqrt{\frac{3}{2}}-1\right], \quad y=-s^{-1}\left[\sqrt{\frac{3}{2}}-1\right],
$$


then $\left|2 s(x+y)-2 s^{2} x y\right|=1$ and the sum of the absolute values of the terms in the logarithmic power series of (2.36) diverge.

In summary, we have shown that $-\sum_{\substack{q=1 \\ m+n=q}}^{\infty} \log \left(1+F_{m, n} x^{m} y^{n}\right)$ will be absolutely convergent whenever $(x, y) \in \mathbb{D}$ or whenever $(x, y) \in D_{x \rho} \times D_{y \rho}$ with $\rho<s^{-1}\left[\sqrt{\frac{3}{2}}-1\right]$, where

$$
D_{x \rho}: \equiv\{x:|x|<\rho\}, \quad D_{y \rho}: \equiv\{y:|y|<\rho\} .
$$

Hence, $\prod_{\substack{q=1 \\ m+n=q}}^{\infty}\left(1+F_{m, n} x^{m} y^{n}\right)^{-1}$ will also be absolutely convergent for the same regions.

We claim this information provides a lower bound on the range of absolute convergence for IPPE2 of Equation (2.27). To determine the domain of convergence of the IPPE2 of Equation (2.27), we must determine the domain of convergence of $\log \prod_{\substack{q=1 \\ m+n=q}}^{\infty}\left(1-h_{m, n} x^{m} y^{n}\right)^{-1}:=-\sum_{\substack{q=1 \\ m+n=q}}^{\infty} \log \left(1-h_{m, n} x^{m} y^{n}\right)$, where the right hand side is defined via the convergence of the double series $-\sum_{\substack{q=1 \\ m+n=q}}^{\infty} \sum_{\ell=1}^{\infty} \frac{\left(h_{m, n} x^{m} y^{n}\right)^{\ell}}{\ell}$. However,

$$
\begin{aligned}
\left|\log \prod_{\substack{q=1 \\
m+n=q}}^{\infty}\left(1-h_{m, n} x^{m} y^{n}\right)^{-1}\right| & =\left|-\sum_{\substack{q=1 \\
m+n=q}}^{\infty} \log \left(1-h_{m, n} x^{m} y^{n}\right)\right| \\
& \leq \sum_{\substack{q=1 \\
m+n=q}}^{\infty}\left|\log \left(1-h_{m, n} x^{m} y^{n}\right)\right| \\
& =\sum_{\substack{q=1 \\
m+n=q}}^{\infty}\left|-\sum_{\ell=1}^{\infty} \frac{\left(h_{m, n} x^{m} y^{n}\right)^{\ell}}{\ell}\right| \\
& \leq \sum_{\substack{q=1 \\
m+n=q}}^{\infty} \sum_{\ell=1}^{\infty} \frac{\left(\left|h_{m, n}\right||x|^{m}|y|^{n}\right)^{\ell}}{\ell} \\
& \leq \sum_{\substack{q=1 \\
m+n=q}}^{\infty} \sum_{\ell=1}^{\infty} \frac{\left(F_{m, n}|x|^{m}|y|^{n}\right)^{\ell}}{\ell}
\end{aligned}
$$


where the last inequality follows from Theorem 2.4.1. These calculations imply that if $\sum_{\substack{q=1 \\ m+n=q}}^{\infty} \log \left(1+F_{m, n} x^{m} y^{n}\right)$ is absolutely convergent, then so are $\sum_{\substack{q=1 \\ m+n=q}}^{\infty} \log (1-$ $\left.h_{m, n} x^{m} y^{n}\right)$ and $\prod_{\substack{q=1 \\ m+n=q}}^{\infty}\left(1-h_{m, n} x^{m} y^{n}\right)^{-1}$.

We can therefore summarize what we have shown so far in the following theorem.

Theorem 2.4.2. (i.) Let $f(x, y)=1+\sum_{\substack{p=1 \\ m+n=p}}^{\infty} a_{m, n} x^{m} y^{n}$. Define $s: \equiv \sup _{\substack{p=1 \\ m+n=p}}\left|a_{m, n}\right|^{\frac{1}{m+n}}$. Then both $f(x, y)$ and its IPPE2,

$$
f(x, y)=1+\sum_{\substack{p=1 \\ m+n=p}}^{\infty} a_{m, n} x^{m} y^{n}=\prod_{\substack{q=1 \\ m+n=q}}^{\infty}\left(1-h_{m, n} x^{m} y^{n}\right)^{-1},
$$

and the auxiliary function, along with its IPPE2

$$
D(x, y)=1-\sum_{\substack{p=1 \\ m+n=p}}^{\infty} s^{m+n} x^{m} y^{n}=\prod_{\substack{q=1 \\ m+n=q}}^{\infty}\left(1+F_{m, n} x^{m} y^{n}\right)^{-1}
$$

will be absolutely convergent whenever $(x, y) \in \mathbb{D}$, where

$$
\mathbb{D}=\left\{(x, y) \in \mathbb{C}^{2}: 2 s(|x|+|y|)+2 s^{2}|x||y|<1\right\} .
$$

See Figure 2.1.

(ii.) With the same conventions as in Part (i.), both $f(x, y)$ and its IPPE2, along with $D(x, y)$ and its IPPE2, will be absolutely convergent whenever $(x, y) \in$ $D_{x \rho} \times D_{y \rho}$ with $\rho<s^{-1}\left[\sqrt{\frac{3}{2}}-1\right]$, where

$$
D_{x \rho}: \equiv\{x:|x|<\rho\}, \quad D_{y \rho}: \equiv\{y:|y|<\rho\} \text {. }
$$

\subsection{Asymptotic Approximation for the Majorizing Inverse Power Product Expansion}

We now derive an asymptotic representation for the majorizing inverse product expansion associated with $1-\sum_{\substack{p=1 \\ m+n=p}}^{\infty} s^{m+n} x^{n} y^{n}, \quad$ where $\quad s: \equiv \sup _{\substack{p=1 \\ m+n=p}}\left|a_{m, n}\right|^{\frac{1}{m+n}}$. Since 
our asymptotic approximation will depend on Equation (2.10), we first need to derive an asymptotic formula for the Taylor series coefficients of $\log \left(1-\sum_{\substack{p=1 \\ m+n=p}}^{\infty} s^{m+n} x^{n} y^{n}\right)$. This calculation will utilize the asymptotic formula (1.53).

$$
d_{M, N}=-\frac{2^{N} s^{M+N} N^{M-1}}{M !}[1+\Delta(M, N)],
$$

which was driven in Section 1.5, where $M \in \mathbb{N}$ is fixed and $\Delta(M, N) \rightarrow 0$ as $N \rightarrow \infty$.

Let $(M, N) \in \mathbb{N} \times \mathbb{N}$. Arrange $\left(h_{M, N}\right)$ in an infinite array as shown in Table 2.1. Then for fixed $M \in \mathbb{N}$, the limit $\lim _{N \rightarrow \infty} h_{M, N}$ is along the $M$-th row of Table 2.1.

$\begin{array}{cccc}h_{1,1} & h_{1,2} & h_{1,3} & \cdots \\ h_{2,1} & h_{2,2} & h_{2,3} & \cdots \\ h_{3,1} & h_{3,2} & h_{3,3} & \cdots \\ h_{4,1} & h_{4,2} & h_{4,3} & \cdots \\ \vdots & \vdots & \vdots & \ddots\end{array}$

Table 2.1: The row asymptotics are calculated in Theorem 2.5.1

For fixed $M \in \mathbb{N}$, Equation (2.39) implies that given $\varepsilon>0$, there exists a positive integer $N_{0}$ such that for $N \geq N_{0},|\Delta(M, N)|<\varepsilon$. For fixed $M$ and arbitrary $\varepsilon>0$, define

$$
q_{\varepsilon}(M):=\max \left\{\varepsilon,|\Delta(M, 1)|,|\Delta(M, 2)|, \ldots,\left|\Delta\left(M, N_{0}-1\right)\right|\right\} .
$$

Equation (1.51) shows that for each $\varepsilon, q_{\varepsilon}(M)$ is a finite positive number.

Next define

$$
Q_{\varepsilon}(M):=\max \left\{q_{\varepsilon}(i)\right\}, \quad 1 \leq i \leq M .
$$

Since $\varepsilon$ is arbitrary, Equation (2.40) proves the following corollary:

Corollary 2.5.1. Let $f(x, y)$ and $\log (f(x, y))$ be as defined in Theorem 1.5.1. Then

$$
d_{M, N}=-\frac{2^{N} s^{M+N} N^{M-1}}{M !}[1+\Delta(M, N)],
$$

where $\Delta(M, N) \rightarrow 0$ as $N \rightarrow \infty$. Furthermore, for fixed $M \in \mathbb{N}$, there exists a positive real number $Q(M)$ such that for all $1 \leq m \leq M$

$$
|\Delta(m, N)| \leq Q(M) .
$$


Corollary (2.5.1) is crucial to proving that the modulus of the coefficients of the majorizing IPPE2 are less than or equal to a constant times the modulus of the corresponding coefficient in the logarithmic expansion.

Lemma 2.5.1. Let $s>0$,

$$
f(x, y)=1-\sum_{\substack{p=1 \\ m+n=p}}^{\infty} s^{m+n} x^{m} y^{n}=\prod_{\substack{p=1 \\ M+N=p}}^{\infty}\left(1-h_{M, N} x^{M} y^{N}\right)^{-1},
$$

and

$$
\log (f(x, y))=\sum_{\substack{p=1 \\ M+N=p}}^{\infty} d_{M, N} x^{M} y^{N} .
$$

For fixed $M \in \mathbb{N}$, there exists a positive integer $N_{0}(M)$ such that for all $N \geq N_{0}(M)$, there exists a positive real number $C(M) \geq 1$, independent of $N$, such that

$$
\left|h_{M, N}\right| \leq C(M)\left|d_{M, N}\right|
$$

Proof. Fix $M$. Equation (2.10) implies that

$$
d_{M, N}=h_{M, N}+\sum_{1 \leq m \leq\left\lfloor\frac{M}{2}\right\rfloor} \frac{m}{M}\left(d_{m, \frac{N m}{M}}\right)^{\frac{M}{m}} \frac{\left(h_{m, \frac{N m}{M}}\right)^{\frac{M}{m}}}{\left(d_{m, \frac{N m}{M}}\right)^{\frac{M}{m}}}
$$

or equivalently that

$$
\frac{h_{M, N}}{d_{M, N}}=1-\sum_{1 \leq m \leq\left\lfloor\frac{M}{2}\right\rfloor} \frac{m}{M} \frac{\left(d_{m, \frac{N m}{M}}\right)^{\frac{M}{m}}}{d_{M, N}} \cdot r_{m, \frac{N m}{M}}^{\frac{M}{m}}, \quad r_{m, \frac{N m}{M}}:=\frac{h_{m, \frac{N m}{M}}}{d_{m, \frac{N m}{M}}}
$$

Claim 1: For all $m \mid M$

$$
\frac{\left(d_{m, \frac{N m}{M}}\right)^{\frac{M}{m}}}{d_{M, N}} \rightarrow 0, \quad \text { as } N \rightarrow \infty .
$$

Proof of Claim 1: By Equation (2.41) we have

$$
\begin{aligned}
d_{m, \frac{N m}{M}} & =-\frac{2^{\frac{N m}{M}}\left(\frac{N m}{M}\right)^{m-1} s^{m+\frac{N m}{M}}}{m !}\left[1+\Delta\left(m, \frac{N m}{M}\right)\right], \\
d_{M, N} & =-\frac{2^{N} N^{M-1} s^{M+N}}{M !}[1+\Delta(M, N)],
\end{aligned}
$$


where $\Delta(M, N) \rightarrow 0$ as $N \rightarrow \infty$. Furthermore, there exists $Q(M)>0$ such that

$$
|\Delta(M, N)| \leq Q(M), \quad\left|\Delta\left(m, \frac{N m}{M}\right)\right| \leq Q(M) ;
$$

see Corollary (2.5.1) and Equation (2.42). Then

$$
\left|\frac{\left(d_{m, \frac{N m}{M}}\right)^{\frac{M}{m}}}{d_{M, N}}\right|=\frac{N^{\frac{(m-1) M}{m}}}{N^{M-1}} \cdot\left|\frac{\left[1+\Delta\left(m, \frac{N m}{M}\right)\right]^{\frac{M}{m}}}{[1+\Delta(M, N)]}\right| \cdot \frac{M !\left(\frac{m}{M}\right)^{\frac{M(m-1)}{m}}}{(m !)^{\frac{M}{m}}} .
$$

We must analyze the behavior of the three factors on the right side (2.47). We begin with the right factor. Since $1 \leq m \leq\left\lfloor\frac{M}{2}\right\rfloor$, we have $1 \leq m$ ! and

$$
2 \leq \frac{M}{m} \leq M, \quad 0<\frac{1}{M} \leq \frac{m}{M} \leq \frac{1}{2}<1, \quad \frac{M(m-1)}{m}<M .
$$

and we deduce that

$$
0<\frac{M !\left(\frac{m}{M}\right)^{\frac{M(m-1)}{m}}}{(m !)^{\frac{M}{m}}} \leq M ! .
$$

We next look at the middle factor of (2.47). Note that

$$
\begin{aligned}
\left|\frac{\left[1+\Delta\left(m, \frac{N m}{M}\right)\right]^{\frac{M}{m}}}{[1+\Delta(M, N)]}\right| & \leq \frac{\left(1+\left|\Delta\left(m, \frac{N m}{M}\right)\right|\right)^{\frac{M}{m}}}{1-|\Delta(M, N)|} \\
& \leq \frac{\left(1+\left|\Delta\left(m, \frac{N m}{M}\right)\right|\right)^{M}}{1-|\Delta(M, N)|} \\
& \leq \frac{(1+Q(M))^{M}}{1-|\Delta(M, N)|}
\end{aligned}
$$

Since $|\Delta(M, N)| \rightarrow 0$, there exists a positive integer $\tilde{N}_{0}$ such that for all $N \geq \tilde{N}_{0}$, we have $|\Delta(M, N)| \leq \frac{1}{2}$, which in turn implies $\frac{1}{2} \leq 1-|\Delta(M, N)|$. Thus, for $N \geq \tilde{N}_{0}$

$$
\left|\frac{\left[1+\Delta\left(m, \frac{N m}{M}\right)\right]^{\frac{M}{m}}}{[1+\Delta(M, N)]}\right| \leq \frac{(1+Q(M))^{M}}{1-|\Delta(M, N)|} \leq 2(1+Q(M))^{M} .
$$


Finally, we look at the left factor of (2.47). Since $2 \leq \frac{M}{m} \leq M$, we have

$$
\frac{N^{\frac{(m-1) M}{m}}}{N^{M-1}}=\frac{1}{N^{M-1-\left(1-\frac{1}{m}\right) M}}=\frac{1}{N^{\frac{M}{m}-1}} \leq \frac{1}{N} .
$$

By placing (2.48) through (2.50) into (2.47), we see that for large $N \geq N_{0}$,

$$
\left|\frac{\left(d_{m, \frac{N m}{M}}\right)^{\frac{M}{m}}}{d_{M, N}}\right| \leq \frac{2 M !}{N^{\frac{M}{m}-1}}(1+Q(M))^{M}
$$

and since $\frac{2 M !}{N^{\frac{M}{m}-1}}(1+Q(M))^{M} \rightarrow 0$ as $N \rightarrow \infty$, we have proven Claim 1 .

Claim 2: For fixed $M$ and $N \geq N_{0}(M)$, there exist a positive $C(M) \geq 1$, a constant independent of $N$ such that

$$
\left|r_{M, N}\right|=\left|\frac{h_{M, N}}{d_{M, N}}\right| \leq C(M) .
$$

The proof of Claim 2 will also prove Equation (2.43).

Proof of Claim 2: To prove Claim 2, we use Claim 1, Equation (2.10), and induction on $M$. For $M=1$, Equation (2.10) shows that $C(1)=1$ since $h_{1, N}=d_{1, N}$. For $M=2$, Equation (2.10) shows that

$$
\begin{aligned}
\frac{h_{2, N}}{d_{2, N}} & =1-\frac{1}{2} \frac{\left(h_{1, \frac{N}{2}}\right)^{2}}{d_{2, N}} \\
& =1-\frac{1}{2} \frac{\left(h_{1, \frac{N}{2}}\right)^{2}}{\left(d_{1, \frac{N}{2}}\right)^{2}} \frac{\left(d_{1, \frac{N}{2}}\right)^{2}}{d_{2, N}}=1-\frac{1}{2} \frac{\left(d_{1, \frac{N}{2}}\right)^{2}}{d_{2, N}}
\end{aligned}
$$

By Claim 1, we know that

$$
\frac{\left(d_{1, \frac{N}{2}}\right)^{2}}{d_{2, N}} \rightarrow 0, \quad \text { as } N \rightarrow \infty
$$


This implies that there exists a positive integer $N_{0}(2)$ such that for all $N \geq N_{0}(2)$, there exists $\tilde{C}(2)>0$ such that

$$
\left|\frac{\left(d_{1, \frac{N}{2}}\right)^{2}}{d_{2, N}}\right| \leq \tilde{C}(2)
$$

Then we define $C(2)=1+\frac{1}{2} \tilde{C}(2)$ since

$$
\left|\frac{h_{2, N}}{d_{2, N}}\right| \leq 1+\frac{1}{2}\left|\frac{\left(d_{1, \frac{N}{2}}\right)^{2}}{d_{2, N}}\right| \leq 1+\frac{1}{2} \tilde{C}(2) .
$$

Now we assume that (2.51) is true for all $m<M$. Equation (2.44) implies that

$$
\begin{aligned}
\left|\frac{h_{M, N}}{d_{M, N}}\right| & \leq 1+\sum_{1 \leq m \leq\left\lfloor\frac{M}{2}\right\rfloor} \frac{m}{M}\left|\frac{\left(d_{m, \frac{N m}{M}}\right)^{\frac{M}{m}}}{d_{M, N}}\right| \cdot\left|\frac{h_{m, \frac{N m}{M}}}{d_{m, \frac{N m}{M}}}\right|^{\frac{M}{m}} \\
& \leq 1+\sum_{1 \leq m \leq\left\lfloor\frac{M}{2}\right\rfloor}\left|\frac{\left(d_{m, \frac{N m}{M}}\right)^{\frac{M}{m}}}{d_{M, N}}\right| \cdot\left|\frac{h_{m, \frac{N m}{M}}}{d_{m, \frac{N m}{M}}}\right|^{\frac{M}{m}} .
\end{aligned}
$$

By Claim 1, there exists a positive integer $N_{0}(M)$ such that for all $N \geq N_{0}(M)$, $\left|\frac{\left(d_{m, \frac{N m}{M}}\right)^{\frac{M}{m}}}{d_{M, N}}\right| \leq \tilde{C}(M)$. Hence, the preceding line becomes
\[ \left|\frac{h_{M, N}}{d_{M, N}}\right| \leq 1+\sum_{1 \leq m \leq\left\lfloor\frac{M}{2}\right\rfloor} \tilde{C}(M)\left|\frac{h_{m, \frac{N m}{M}}}{d_{m, \frac{N m}{M}}}\right|^{\frac{M}{m}} . \]

By the induction hypothesis, there exists $N_{0}(M)=\max \left\{N_{0}(1), \ldots, N_{0}(m)\right\}$ such that for each $1 \leq m \leq\left\lfloor\frac{M}{2}\right\rfloor$ and $N \geq N_{0}(M),\left|\frac{h_{m, \frac{N m}{M}}}{d_{m, \frac{N m}{M}}}\right| \leq C(m)$. For $1 \leq m \leq\left\lfloor\frac{M}{2}\right\rfloor$, define $\tilde{\mathscr{C}}(M):=\max \{C(m)\}$. Then (2.53) becomes

$$
\left|\frac{h_{M, N}}{d_{M, N}}\right| \leq 1+\sum_{1 \leq m \leq\left\lfloor\frac{M}{2}\right\rfloor} \tilde{C}(M)(\tilde{\mathscr{C}}(M))^{\frac{M}{m}} \leq 1+M \tilde{C}(M)(\tilde{\mathscr{C}}(M))^{M}
$$


If we set $C(M)=1+M \tilde{C}(M)(\tilde{\mathscr{C}}(M))^{M}$, we have verified Claim 2, and hence Equation (2.43), which completes the proof.

By using Equation (2.39) and Lemma (2.5.1) we are in a position to state and prove the main theorem of this section.

Theorem 2.5.1. Let $f(x, y)=1-\sum_{\substack{p=1 \\ m+n=p}}^{\infty} s^{m+n} x^{m} y^{n}=\frac{1-2 s(x+y)+2 s^{2} x y}{(1-s x)(1-s y)}$ where $s>0$. For this special function $f(x, y)$ and its associated IPPE2 $\prod_{\substack{p=1 \\ M+N=p}}^{\infty}\left(1-h_{M, N} x^{M} y^{N}\right)^{-1}$, we have

$$
-h_{M, N} \sim-d_{M, N} \sim s^{M+N} \sum_{q=N}^{N+M} \frac{2^{q}(q-1) !(-1)^{M+N-q}}{(q-M) !(q-N) !(M+N-q) !},
$$

as $N \rightarrow \infty$, where $M$ is a fixed element of $\mathbb{N}$ and $(M, N) \in \mathbb{N} \times \mathbb{N}$.

Proof. Equation (2.10) implies that

$$
-h_{M, N}=-d_{M, N}+\underbrace{\frac{1}{M} \sum_{\substack{m \mid M \\ 1 \leq m \leq\left\lfloor\frac{M}{2}\right\rfloor}} m\left(h_{m, \frac{N m}{M}}\right)}_{:=\Delta} .
$$

We ultimately want to show

$$
\frac{\Delta}{-d_{M, N}} \rightarrow 0, \quad \text { as } N \rightarrow \infty
$$

By Equation (2.39) and Lemma (2.5.1), we observe that

$$
\begin{aligned}
|\Delta| & =\left|\frac{1}{M} \sum_{\substack{m \mid M \\
1 \leq m \leq\left\lfloor\frac{M}{2}\right\rfloor}} m\left(h_{m, \frac{N m}{M}}\right)^{\frac{M}{m}}\right| \leq \frac{1}{M} \sum_{\substack{m \mid M \\
1 \leq m \leq\left\lfloor\frac{M}{2}\right\rfloor}} m\left|h_{m, \frac{N m}{M}}\right|^{\frac{M}{m}} \\
& \leq \frac{1}{M} \sum_{\substack{m \mid M \\
1 \leq m \leq\left\lfloor\frac{M}{2}\right\rfloor}} m\left|C(M) d_{m, \frac{N m}{M}}\right|^{\frac{M}{m}}
\end{aligned}
$$




$$
\begin{aligned}
& =\frac{1}{M} \sum_{\substack{m \mid M \\
1 \leq m \leq\left\lfloor\frac{M}{2}\right\rfloor}} m(C(M))^{\frac{M}{m}}\left[\frac{s^{m}(2 s)^{\frac{N m}{M}}\left(\frac{N m}{M}\right)^{m-1}}{m !}\right]^{\frac{M}{m}}\left|1+\Delta\left(m, \frac{N m}{M}\right)\right|^{\frac{M}{m}} \\
& =\frac{s^{M+N} 2^{N}}{M} \sum_{\substack{m \mid M \\
1 \leq m \leq\left\lfloor\frac{M}{2}\right\rfloor}} \frac{m(C(M))^{\frac{M}{m}}\left(\frac{m}{M}\right)^{(m-1) \frac{M}{m}}}{(m !)^{\frac{M}{m}}} N^{(m-1) \frac{M}{m}}\left|1+\Delta\left(m, \frac{N m}{M}\right)\right|^{\frac{M}{m}} .
\end{aligned}
$$

Hence, as $N \rightarrow \infty$, since

$$
\begin{aligned}
& \left|\frac{|\Delta|}{-d_{M, N}}\right|=\sum_{\substack{m \mid M \\
1 \leq m \leq\left\lfloor\frac{M}{2}\right\rfloor}} \frac{m(C(M))^{\frac{M}{m}}\left(\frac{m}{M}\right)^{(m-1) \frac{M}{m}} M !}{(m !)^{\frac{M}{m}} M} \cdot \frac{N^{(m-1) \frac{M}{m}}}{N^{M-1}}\left|\frac{\left[1+\Delta\left(m, \frac{N m}{M}\right)\right]^{\frac{M}{m}}}{[1+\Delta(M, N)]}\right| \\
& \leq 2[1+Q(M)]^{M} \sum_{\substack{m \mid M \\
1 \leq m \leq\left\lfloor\frac{M}{2}\right\rfloor}} \frac{m(C(M))^{\frac{M}{m}}\left(\frac{m}{M}\right)^{(m-1) \frac{M}{m}} M !}{(m !)^{\frac{M}{m}} M} \cdot \frac{N^{(m-1) \frac{M}{m}}}{N^{M-1}}, \quad \text { by }(2.49) \\
& \leq 2[1+Q(M)]^{M} \sum_{\substack{m \mid M \\
1 \leq m \leq\left\lfloor\frac{M}{2}\right\rfloor}} \frac{(C(M))^{M}(M-1) !}{N^{\frac{M}{m}-1}}, \quad \text { since } C(M) \geq 1 \\
& \leq 2[1+Q(M)]^{M} \sum_{\substack{m \mid M \\
1 \leq m \leq\left\lfloor\frac{M}{2}\right\rfloor}}(C(M))^{M}(M-1) ! \cdot \max \left(\frac{1}{N^{\frac{M}{m}-1}}\right) \\
& \leq \frac{2[1+Q(M)]^{M}}{N} \sum_{\substack{m \mid M \\
1 \leq m \leq\left\lfloor\frac{M}{2}\right\rfloor}}(C(M))^{M}(M-1) ! \\
& \leq \frac{M ![1+Q(M)]^{M}(C(M))^{M}}{N},
\end{aligned}
$$

and $\frac{M ![1+Q(M)]^{M}(C(M))^{M}}{N} \rightarrow 0$ as $N \rightarrow \infty$. Thus, Equation (2.57) is true.

We return to Equation (2.56), apply (2.57), and discover that

$$
\begin{aligned}
-h_{M, N} & =-d_{M, N}+\Delta \\
& =-d_{M, N}\left[1-\frac{\Delta}{-d_{M, N}}\right] \rightarrow-d_{M, N} \quad \text { as } \quad N \rightarrow \infty .
\end{aligned}
$$




\subsection{Combinatorial Interpretations for IPPE2}

In this section we develop combinatorial interpretations for Equations (2.4) and (2.5). Our first two interpretations involve generating functions for sequences of vector partitions. Let $p_{d}((m, n))$ be the number of partitions of $(m, n)$ with distinct parts; see Definition 1.2.3. The generating function is given by

$$
\prod_{\substack{q=1 \\ m+n=q}}^{\infty}\left(1+x^{m} y^{n}\right)=\sum_{\substack{p=0 \\ m+n=p}}^{\infty} p_{d}((m, n)) x^{m} y^{n} .
$$

If $h_{m, n}=1$, Equation (2.5) becomes

$$
\prod_{\substack{q=1 \\ m+n=q}}^{\infty}\left(1-x^{m} y^{n}\right)^{-1}=\sum_{\substack{p=0 \\ m+n=p}}^{\infty} p((m, n)) x^{m} y^{n}
$$

where $p((m, n))$ is the number of vector partitions of $(m, n)$.

Equations (2.58) and (2.59) interpret the product side of Equations (2.4) and (2.5) respectively. Next we give a combinatorial interpretation when we start with the sum. Define

$$
f(x, y)=1-\sum_{\substack{p=1 \\ m+n=p}}^{\infty} x^{m} y^{n}=\prod_{\substack{p=1 \\ m+n=p}}^{\infty}\left(1-g_{m, n} x^{m} y^{n}\right),
$$

where $g_{m, n} \in \mathbb{N}$. Take Equation (2.60) and form the reciprocal.

$$
\frac{1}{1-\sum_{\substack{p=1 \\ m+n=p}}^{\infty} x^{m} y^{n}}=\frac{1}{\prod_{\substack{p=1 \\ m+n=p}}^{\infty}\left(1-g_{m, n} x^{m} y^{n}\right)}=\prod_{\substack{p=1 \\ m+n=p}}^{\infty}\left(1-h_{m, n} x^{m} y^{n}\right)^{-1}
$$

where $h_{m, n}=g_{m, n}$.

Expand the left side of Equation (2.61) as 


$$
\begin{aligned}
& \frac{1}{1-\sum_{\substack{p=1 \\
m+n=p}}^{\infty} x^{m} y^{n}}=\frac{1}{1-\left[\frac{1-(1-x)(1-y)}{(1-x)(1-y)}\right]} \\
& =1+\sum_{\substack{p=1 \\
m+n=p}}^{\infty} x^{m} y^{n}+\left[\sum_{\substack{p=1 \\
m+n=p}}^{\infty} x^{m} y^{n}\right]^{2}+\cdots+\left[\sum_{\substack{p=1 \\
m+n=p}}^{\infty} x^{m} y^{n}\right]^{k}+\ldots \\
& =1+\sum_{\substack{p=1 \\
m+n=p}}^{\infty} \hat{C}(m, n, 1) x^{m} y^{n}+\sum_{\substack{p=2 \\
m+n=p}}^{\infty} \hat{C}(m, n, 2) x^{m} y^{n}+\sum_{\substack{p=3 \\
m+n=p}}^{\infty} \hat{C}(m, n, 3) x^{m} y^{n}+\ldots \\
& =1+\sum_{\substack{p=1 \\
m+n=p}}^{\infty} C(m, n) x^{m} y^{n}, \quad C(m, n):=\sum_{l} \hat{C}(m, n, l) .
\end{aligned}
$$

Because $\hat{C}(m, n, k)=0$ whenever $m+n<k$, we observe that $C(m, n)$ is a finite sum.

We want to determine a closed form $\hat{C}(m, n, k) x^{m} y^{n}$ whenever $k \geq 1$. Clearly $\hat{C}(m, n, 1)=1$ whenever $m, n \neq 0$. If $k=2$, we find that

$$
\left[\sum_{\substack{p=1 \\
m+n=1}}^{\infty} x^{m} y^{n}\right]^{2}=\sum_{\substack{\ell=2 \\
m+n=\ell}}^{\infty} \hat{C}(m, n, 2) x^{m} y^{n}=\sum_{\substack{\ell=2 \\
s+t=\ell}}^{\infty}\left[\left(\begin{array}{c}
m+1 \\
1
\end{array}\right)\left(\begin{array}{c}
n+1 \\
1
\end{array}\right)-2\right] x^{m} y^{n}
$$

since a monomial of the form $x^{m} y^{n}$ is obtained as a product of two terms of $x^{m_{1}} y^{n_{1}} x^{m_{2}} y^{n_{2}}$, where $m_{2}=m-m_{1}$ and $n_{2}=n-n_{1}$. Clearly, $0 \leq m_{1} \leq m$ and $0 \leq n_{1} \leq n$. This would suggest that the answer is $(m+1)(n+1)$. But there are two possibilities that we can not have, namely $x^{0} y^{0}$ and $x^{m} y^{n}$. Thus, there are $(m+1)(n+1)-2$ ways to write $x^{m} y^{n}$ as a product of two terms from the original series.

Define an ordered pair composition of $(m, n)$ with at most two non-negative parts to be an ordered pair $\left(c_{i}, c_{i}^{\prime}\right)$ such that $c_{i}=c_{i_{1}}+c_{i_{2}}=m$ and $c_{i}^{\prime}=c_{i_{1}}^{\prime}+c_{i_{2}}^{\prime}=n$ (with $c_{i_{1}}, c_{i_{2}}, c_{i_{1}}^{\prime}, c_{i_{2}}^{\prime} \in \mathbb{N}_{0}$ ). To obtain the monomial $x^{m} y^{n}$, we chose $x^{c_{i_{1}}} y^{c_{i_{1}}^{\prime}}$ from one factor of $\sum_{\substack{p=1 \\ m+n=1}}^{\infty} x^{m} y^{n}$ and $x^{c_{i_{2}}} y^{c_{i_{2}}^{\prime}}$ from the other factor. Then $\left(\begin{array}{c}m+1 \\ 1\end{array}\right)\left(\begin{array}{c}n+1 \\ 1\end{array}\right)-2$ counts the number of ordered pair compositions of $(m, n)$ with exactly two parts, 
which satisfy the extra condition that $c_{i_{p}}$ and $c_{i_{p}}^{\prime}$ are never simultaneously zero for $p=1,2$. For example, $\hat{C}(3,2,2)=10$ since

$$
\begin{aligned}
& \left(c_{1}, c_{1}^{\prime}\right)=(3+0,0+2)=x^{3} \cdot y^{2},\left(c_{2}, c_{2}^{\prime}\right)=(3+0,1+1)=x^{3} y \cdot y, \\
& \left(c_{3}, c_{3}^{\prime}\right)=(2+1,0+2)=x^{2} \cdot x y^{2},\left(c_{4}, c_{4}^{\prime}\right)=(2+1,1+1)=x^{2} y \cdot x y \\
& \left(c_{5}, c_{5}^{\prime}\right)=(2+1,2+0)=x^{2} y^{2} \cdot y, \quad\left(c_{6}, c_{6}^{\prime}\right)=(1+2,0+2)=x \cdot x^{2} y^{2}, \\
& \left(c_{7}, c_{7}^{\prime}\right)=(1+2,1+1)=x y \cdot x^{2} y,\left(c_{8}, c_{8}^{\prime}\right)=(1+2,2+0)=x y^{2} \cdot x^{2} \\
& \left(c_{9}, c_{9}^{\prime}\right)=(0+3,2+0)=y^{2} \cdot x^{3},\left(c_{10}, c_{10}^{\prime}\right)=(0+3,1+1)=y \cdot x^{3} y .
\end{aligned}
$$

Note that we can not obtain $(3+0,2+0)$ (corresponding to $\left.x^{3} y^{2} \cdot 1\right)$ or $(0+3,0+$ 2) (corresponding to $1 \cdot x^{3} y^{2}$ ).

We use this result to determine $\hat{C}(m, n, 3)$ as follows:

$$
\begin{aligned}
{\left[\sum_{\substack{p=1 \\
m+n=p}}^{\infty} x^{m} y^{n}\right]^{3} } & =\left[\sum_{\substack{p=1 \\
m+n=p}}^{\infty} x^{m} y^{n}\right]^{2}\left[\sum_{\substack{p=1 \\
m+n=p}}^{\infty} x^{m} y^{n}\right]^{1} \\
& =\left[\sum_{\substack{\ell=2 \\
m_{1}+n_{1}=\ell}}^{\infty}\left(\left(m_{1}+1\right)\left(n_{1}+1\right)-2\right) x^{m_{1}} y^{n_{1}}\right]\left[\sum_{\substack{p=1 \\
m_{2}+n_{2}=p}}^{\infty} x^{m_{2}} y^{n_{2}}\right] .
\end{aligned}
$$

Since both of these series are indexed by pairs of integers, this is the same as the previous situation except that not all the coefficients are 1 . For $(m, n) \neq(0,0)$, our preceding coefficient was obtained by:

$$
\sum_{\substack{1 \leq m_{1}+n_{1}<m+n \\ 0 \leq m_{1} \leq m \\ 0 \leq n_{1} \leq n}} 1=(m+1)(n+1)-2 .
$$

This time we have:

$$
\begin{aligned}
& \hat{C}(m, n, 3)=\sum_{\substack{2 \leq m_{1}+n_{1}<m+n \\
0 \leq m_{1} \leq m \\
0 \leq n_{1} \leq n}}\left[\left(m_{1}+1\right)\left(n_{1}+1\right)-2\right] \\
& =\sum_{m_{1}=0}^{m}\left(m_{1}+1\right) \sum_{n_{1}=0}^{n}\left(n_{1}+1\right)-\sum_{m_{1}=0}^{m} \sum_{n_{1}=0}^{n} 2-[(m+1)(n+1)-2]-[-1],
\end{aligned}
$$


where $[(m+1)(n+1)-2]$ reflects the fact that $x^{m_{1}} y^{n_{1}} \neq x^{m} y^{n}$ and -1 reflects the fact that $x^{m_{1}} y^{n_{1}} \neq x^{0} y^{0}$. By applying Identity (1.49) of [25], namely

$$
\sum_{k=0}^{n}\left(\begin{array}{c}
x+k \\
k
\end{array}\right)=\sum_{k=0}^{n}\left(\begin{array}{c}
x+k \\
x
\end{array}\right)=\left(\begin{array}{c}
x+1+n \\
n
\end{array}\right)=\left(\begin{array}{c}
x+1+n \\
x+1
\end{array}\right)
$$

we see that the preceding sum becomes

$$
\hat{C}(m, n, 3)=\left(\begin{array}{c}
m+2 \\
2
\end{array}\right)\left(\begin{array}{c}
n+2 \\
2
\end{array}\right)-3\left(\begin{array}{c}
m+1 \\
1
\end{array}\right)\left(\begin{array}{c}
n+1 \\
1
\end{array}\right)+3
$$

where $\hat{C}(m, n, 3)$ counts the number of ordered pair compositions of $(m, n)$ with at most three non-negative parts such that corresponding parts are never simultaneously zero. In other words, $\hat{C}(m, n, 3)$ counts ordered pairs $\left(c_{i}, c_{i}^{\prime}\right)$, where $c_{i}=c_{i_{1}}+c_{i_{2}}+c_{i_{3}}=m, c_{i}^{\prime}=c_{i_{1}}^{\prime}+c_{i_{2}}^{\prime}+c_{i_{3}}^{\prime}=n$, and for $p \in\{1,2,3\}, c_{i_{p}}$ and $c_{i_{p}}^{\prime}$ are never both zero.

The preceding analysis suggests the following proposition:

Proposition 2.6.1. Let $k$ be a positive integer. For fixed $(m, n) \in \mathbb{N}_{0} \times \mathbb{N}_{0}$, let $\hat{C}(m, n, k)$ count the number of ordered pair compositions of $(m, n)$ with at most $k$ non-negative parts such that the corresponding parts are never simultaneously zero. In other words, $\hat{C}(m, n, k)$ counts the number of ordered pairs $\left(c_{i}, c_{i}^{\prime}\right)$, such that $c_{i}=\sum_{j=1}^{k} c_{i_{j}}=m, c_{i}^{\prime}=\sum_{j=1}^{k} c_{i_{j}}^{\prime}=n$, and for $1 \leq p \leq k, c_{i_{p}}$ and $c_{i_{p}}^{\prime}$ are never both zero. Then,

$$
\left[\sum_{\substack{p=1 \\ m+n=p}}^{\infty} x^{m} y^{n}\right]^{k}=\sum_{\substack{p=k \\ m+n=p}}^{\infty} \hat{C}(m, n, k) x^{m} y^{n}
$$

Furthermore,

$$
\hat{C}(m, n, k)=\sum_{i=0}^{k-1}(-1)^{i}\left(\begin{array}{c}
k \\
i
\end{array}\right)\left(\begin{array}{c}
m+k-1-i \\
k-1-i
\end{array}\right)\left(\begin{array}{c}
n+k-1-i \\
k-1-i
\end{array}\right) .
$$

Proof. The validity of Equation (2.63) follows by analyzing how to create a monomial $x^{m} y^{n}$ from the expansion of the product $\left[\sum_{\substack{p=1 \\ m+n=p}}^{\infty} x^{m} y^{n}\right]^{k}$. Since such a product 
has $k$ factors of the form $\sum_{\substack{p=1 \\ m+n=p}}^{\infty} x^{m} y^{n}$, we select a monomial of the form $x^{c_{i p}} y^{c^{\prime}}$ from each factor where

$$
x^{m} y^{n}=x^{c_{i_{1}}} y^{c_{i_{1}}^{\prime}} \cdot x^{c_{i_{2}}} y^{c_{i_{2}}^{\prime}} \cdots x^{c_{i_{k}}} y^{c_{i_{k}}^{\prime}}=x^{\sum_{j=1}^{k} c_{i_{j}}} y^{\sum_{j=1}^{k} c_{i_{j}}^{\prime}} .
$$

If, for $1 \leq p \leq k, c_{i_{p}}=0=c_{i_{p}}^{\prime}$, then we would have selected $x^{c_{i_{p}}} y^{c_{i_{p}}^{\prime}}=1$ from the $p^{\text {th }}$ factor. But since $\sum_{\substack{p=1 \\ m+n=p}}^{\infty} x^{m} y^{n}=x+y+x^{2}+x y+y^{2}+\ldots$, this is impossible.

It remains to verify Equation (2.64) through induction on $k$. We have already shown the validity of (2.64) for $k=1,2,3$. Now assume that we have proven (2.64) for all positive integers less than or equal to some fixed positive integer $k$. By construction

$$
\begin{aligned}
\sum_{\substack{q=k+1 \\
m+n=q}}^{\infty} \hat{C}(m, n, k+1) x^{m} y^{n} & =\left[\sum_{\substack{p=1 \\
m+n=1}}^{\infty} x^{m} y^{n}\right]^{k+1}=\left[\sum_{\substack{p=1 \\
m+n=1}}^{\infty} x^{m} y^{n}\right]^{k}\left[\sum_{\substack{p=1 \\
m+n=1}}^{\infty} x^{m} y^{n}\right] \\
& =\left[\sum_{\substack{q=k \\
m+n=q}}^{\infty} \hat{C}(m, n, k) x^{m} y^{n}\right]\left[\sum_{\substack{q=1 \\
m+n=q}}^{\infty} x^{m} y^{n}\right] .
\end{aligned}
$$

Coefficient comparison on the preceding line implies that

$$
\begin{aligned}
& \hat{C}(m, n, k+1)=\sum_{\substack{k \leq m_{1}+n_{1}<m+n \\
0 \leq m_{1} \leq m \\
0 \leq n_{1} \leq n}} \hat{C}(m, n, k) \\
& =\sum_{m_{1}=0}^{m} \sum_{n_{1}=0}^{n} \sum_{i=0}^{k-1}(-1)^{i}\left(\begin{array}{c}
k \\
i
\end{array}\right)\left(\begin{array}{c}
m_{1}+k-1-i \\
k-1-i
\end{array}\right)\left(\begin{array}{c}
n_{1}+k-1-i \\
k-1-i
\end{array}\right) \\
& -\sum_{i=0}^{k-1}(-1)^{i}\left(\begin{array}{c}
k \\
i
\end{array}\right)\left(\begin{array}{c}
m+k-1-i \\
k-1-i
\end{array}\right)\left(\begin{array}{c}
n+k-1-i \\
k-1-i
\end{array}\right) \\
& -\sum_{i=0}^{k-1}(-1)^{i}\left(\begin{array}{c}
k \\
i
\end{array}\right)\left(\begin{array}{c}
k-1-i \\
k-1-i
\end{array}\right)\left(\begin{array}{c}
k-1-i \\
k-1-i
\end{array}\right),
\end{aligned}
$$

where the last equality follows by the induction hypothesis and the subtracted terms respectively reflect the fact that the first factor can not contribute $x^{m} y^{n}$ or 
$x^{0} y^{0}$. To simplify the first sum of (2.65) observe that

$$
\begin{aligned}
& \sum_{m_{1}=0}^{m} \sum_{n_{1}=0}^{n} \sum_{i=0}^{k-1}(-1)^{i}\left(\begin{array}{c}
k \\
i
\end{array}\right)\left(\begin{array}{c}
m_{1}+k-1-i \\
k-1-i
\end{array}\right)\left(\begin{array}{c}
n_{1}+k-1-i \\
k-1-i
\end{array}\right) \\
= & \sum_{i=0}^{k-1}(-1)^{i}\left(\begin{array}{c}
k \\
i
\end{array}\right) \sum_{m_{1}=0}^{m}\left(\begin{array}{c}
m_{1}+k-1-i \\
k-1-i
\end{array}\right) \sum_{n_{1}=0}^{n}\left(\begin{array}{c}
n_{1}+k-1-i \\
k-1-i
\end{array}\right) \\
= & \sum_{i=0}^{k-1}(-1)^{i}\left(\begin{array}{c}
k \\
i
\end{array}\right)\left(\begin{array}{c}
m+k-i \\
k-i
\end{array}\right)\left(\begin{array}{c}
n+k-i \\
k-i
\end{array}\right),
\end{aligned}
$$

where the last equality follows from Identity (1.49) of [25]. To simplify the second sum of (2.65) observe that

$$
\begin{aligned}
& \sum_{i=0}^{k-1}(-1)^{i}\left(\begin{array}{c}
k \\
i
\end{array}\right)\left(\begin{array}{c}
m+k-1-i \\
k-1-i
\end{array}\right)\left(\begin{array}{c}
n+k-1-i \\
k-1-i
\end{array}\right) \\
& =\sum_{i=1}^{k}(-1)^{i-1}\left(\begin{array}{c}
k \\
i-1
\end{array}\right)\left(\begin{array}{c}
m+k-i \\
k-i
\end{array}\right)\left(\begin{array}{c}
n+k-i \\
k-i
\end{array}\right) .
\end{aligned}
$$

To simplify the third sum of (2.65), we apply Identity (1.5) of [25], namely

$$
\sum_{k=0}^{n}(-1)^{k}\left(\begin{array}{l}
x \\
k
\end{array}\right)=(-1)^{n-1}\left(\begin{array}{c}
x-1 \\
n
\end{array}\right)
$$

to show that

$$
\sum_{i=0}^{k-1}(-1)^{i}\left(\begin{array}{l}
k \\
i
\end{array}\right)=(-1)^{k-1}\left(\begin{array}{l}
k-1 \\
k-1
\end{array}\right)=(-1)^{k-1}
$$


By subtracting (2.67) and (2.68) from (2.66), we find that

$$
\begin{aligned}
\hat{C}(m, n, k+1)= & \left(\begin{array}{c}
m+k \\
k
\end{array}\right)\left(\begin{array}{c}
n+k \\
k
\end{array}\right)+ \\
& \sum_{i=1}^{k-1}(-1)^{i}\left[\left(\begin{array}{c}
k \\
i
\end{array}\right)+\left(\begin{array}{c}
k \\
i-1
\end{array}\right)\right]\left(\begin{array}{c}
m+k-i \\
k-i
\end{array}\right)\left(\begin{array}{c}
n+k-i \\
k-i
\end{array}\right)+ \\
& (-1)^{k}\left(\begin{array}{c}
k \\
k-1
\end{array}\right)+(-1)^{k} \\
= & \left(\begin{array}{c}
m+k \\
k
\end{array}\right)\left(\begin{array}{c}
n+k \\
k
\end{array}\right)+\sum_{i=1}^{k-1}(-1)^{i}\left(\begin{array}{c}
k+1 \\
i
\end{array}\right)\left(\begin{array}{c}
m+k-i \\
k-i
\end{array}\right)\left(\begin{array}{c}
n+k-i \\
k-i
\end{array}\right)+ \\
& (-1)^{k}(k+1) \\
= & \sum_{i=0}^{k}(-1)^{i}\left(\begin{array}{c}
k+1 \\
i
\end{array}\right)\left(\begin{array}{c}
m+k-i \\
k-i
\end{array}\right)\left(\begin{array}{c}
n+k-i \\
k-i
\end{array}\right)
\end{aligned}
$$

which completes the induction step.

Returning to Equation (2.62), we deduce that $C(m, n)$ counts the number of ordered pair compositions of $(m, n)$ such that identically indexed parts of the individual compositions associated with $m$ and $n$ are never simultaneously zero. Furthermore, Equation (2.62) provides a way of factoring the bivariate Taylor series associated with $(C(m, n))_{m, n}^{\infty}$. 


\section{Chapter 3}

\section{Factorization of Bivariate Matrix Power Series Via Power Product Expansion}

\subsection{Introduction}

Matrix functions occur number theory, combinatorics, linear algebra, and analysis. Typically these matrix functions are represented as power series. But for certain problems, valuable insight is gained by analyzing the power product expansion of the given matrix function. As a case in point, let $A, B \in M_{d}(\mathbb{C})$, and let $\|\cdot\|$ be some matrix norm such that $\|A\| \leq \rho<1$ and $\|B\| \leq \rho<1$. Furthermore, assume that $A B=B A$. Suppose we want an approximation to $(I-A)^{-1}(I-B)^{-1}$. Consider the identities

$$
\begin{aligned}
(I-A)^{-1}(I-B)^{-1} & =I+\sum_{\substack{p=1 \\
m+n=p}}^{\infty} A^{m} B^{n}=\prod_{k=1}^{\infty}\left(I+A^{2^{k}}\right) \prod_{k=1}^{\infty}\left(I+B^{2^{k}}\right) \\
& =\prod_{q=0}^{\infty}\left(I+A^{2^{q}}+(A B)^{2^{q}}+B^{2^{q}}\right)
\end{aligned}
$$

The right side of Equation (3.1) is an example of an matrix power product expansion in two independent variables. Given $F(x, y)=I+\sum_{\substack{p=1 \\ m+n=p}}^{\infty} A_{m, n} x^{m} y^{n}$ with matrix coefficients, where either the defining expression for $F(x, y)$ is treated as a 
formal power series expansion or $F(x, y)$ is an analytic function with $F(0,0)=I$, the right side of

$$
F(x, y)=\prod_{\substack{q=1 \\ m+n=q}}^{\infty}\left(I+G_{m, n} x^{m} y^{n}\right)
$$

is defined to be matrix power product expansion in two independent variables, (denoted MPPE2).

The purpose of this chapter is to obtain both algebraic and analytic theorems for the MPPE2 expansion of $F(x, y)=I+\sum_{\substack{p=1 \\ m+n=p}}^{\infty} A_{m, n} x^{m} y^{n}$. The three main results are as follows:

1. An algebraic structure property for $\left(G_{m, n}\right)$ in terms of recursive "mixed expansions"; see Theorem 3.17.

2. A domain of convergence criteria for the MPPE2 in terms of a "majorizing" infinite product; see Theorem 3.5.1.

3. A domain of convergence criteria for the MPPE2's by norm criteria; see Theorem 3.5.3.

The outline of this chapter is as follows. In Section 2 we introduce and illustrate the main concepts that are needed throughout this work. In Section 3 we study the expansion of a power series into a MPPE2 and provide an algebraic representation for the coefficients $G_{m, n}$ as a multivariate polynomials in $\left(A_{m, n}\right)_{m, n=0}^{\infty}$. In Section 4 we provide another way to recursively express the coefficients $G_{m, n}$ as a multivariate polynomial of the variables $A_{m, n}$. The algebraic result of Section 4 reveals an intriguing property of these expansions. If $A_{m, n} \leq 0$, then the coefficients $G_{m, n}$ in the MPPE2 are non-positive. Section 5 exploits the non-positivity result of Section 4 to determine convergence conditions of the MPPE2 in terms of a majorizing power product by focusing on spectral criteria. Moreover, at the end of Section 5, we employ norm criteria to analyze the convergence of MPPE2. Section 6 is devoted to the study of the matrix MPPE2 induced by scalar functions. Finally, in Section 7 six examples are presented to illustrate the applicability of the section 6 theorems. 


\subsection{Matrix Power Product Expansions}

The goal of this section is twofold: first to define matrix power product expansions and secondly to introduce notational conventions that will be useful for stating the algebraic and analytic results which appear in the following sections. We begin by addressing our first goal of defining a matrix power product expansion. Since such expressions are product expansions of the matrix function $F(x, y)=I+\sum_{\substack{p=1 \\ m+n=p}}^{\infty} A_{m, n} x^{m} y^{n}$, where either the defining expression for $F(x, y)$ is treated as a formal power series expansion or an analytic function with $F(0,0)=I$, it behoves us to first discuss our conventions regarding double summation. Unlike the convergence of the series $\sum_{n=0}^{\infty} A_{n}$, the convergence of the double series $\sum_{m, n=0}^{\infty} B_{m, n}$ requires additional considerations. In order to justify the particular order of summation that we utilize throughout this work, we briefly recall some theoretical results. Following J. Morrow [38], we define $\sum_{m, n} B_{m, n}$ as a double indexed infinite series of complex matrices. (In our particular case, $B_{m, n}=A_{m, n} x^{m} y^{n}$.) We define the associated sequence of partial sums $\left(S_{m, n}\right)$ via the finite sum

$$
S_{m, n}=\sum_{j=1}^{m} \sum_{k=1}^{n} B_{j, k} .
$$

We say $\sum_{m, n} B_{m, n}$ converges if and only if $\lim _{m, n \rightarrow \infty} S_{m, n}$ converges. We say $\sum_{m, n} B_{m, n}$ converges absolutely if and only if $\sum_{m, n}|| B_{m, n} \|$. The crucial result, [[38], Theorem 2], states that if $\sum_{m, n} B_{m, n}$ is absolutely convergent, then $\sum_{m, n} B_{m, n}$ converges and that the sum of $\sum_{m, n} B_{m, n}$ can be computed by any rearrangement of terms. Since we will be working with either doubly indexed formal power series or doubly indexed absolutely convergent series, without loss of generality, we define

$$
\sum_{m, n} B_{m, n}:=B_{0,0}+\sum_{\substack{p=1 \\ m+n=p}}^{\infty} B_{m, n}=B_{0,0}+B_{1,0}+B_{0,1}+B_{2,0}+B_{1,1}+B_{0,2}+\ldots
$$

where the partial ordering uses Definition 1.2.1.

Now that we have a clearly defined protocol regarding the double summation of $F(x, y)=I+\sum_{\substack{p=1 \\ m+n=p}}^{\infty} A_{m, n} x^{m} y^{n}$, we next discuss some notational conventions regarding the matrix coefficients $\left(A_{m, n}\right)_{m, n}$. 
Definition 3.2.1. Let $M_{d}(\mathbb{F})$ denote the set of $d \times d$ matrices over the field $\mathbb{F}$ where $\mathbb{F}=\mathbb{C}$ or $\mathbb{F}=\mathbb{R}$. Given $A \in M_{d}(\mathbb{F})$, we denote $A=\left(a_{u, v}\right)$, where $a_{u, v} \in \mathbb{F}$ for $u, v=1,2, \ldots, d$. Let $I_{d}=\left(\delta_{u, v}\right)$ denote the identity matrix. We may omit the subscript $d$ and denote by $I$ the $d \times d$ identity matrix. Let $O$ denote the matrix with all of its entries 0 . Given $A \in M_{d}(\mathbb{F})$, the matrix $|A| \in M_{d}(\mathbb{R})$ is $|A|=\left(\left|a_{u, v}\right|\right)$, where $u, v=1,2, \ldots, d$. Given $A, B \in M_{d}(\mathbb{R})$, we say $A \leq B$ if and only if $a_{u, v} \leq b_{u, v}$ for $u, v=1,2, \ldots, d$.

Definition 3.2.2. Let $A \in M_{d}(\mathbb{R})$. We say that $A$ is a positive matrix if and only if $a_{u, v}>0$ for $u, v=1,2, \ldots, d$ and write $A>O$. We say that $A$ is a non-negative matrix if and only if $a_{u, v} \geq 0$ for $u, v=1,2, \ldots, d$ and write $A \geq O$. We say that $A$ is a non-positive matrix if and only if $a_{u, v} \leq 0$ for $u, v=1,2, \ldots, d$ and write $A \leq O$. We say that $A$ is a negative matrix if and only if $a_{u, v}<0$ for $u, v=1,2, \ldots, d$ and write $A<O$. If $A$ is either a non-positive or negative matrix, it is equivalent to write $A=-|A|$.

We are now in a position to define what we mean by the matrix power product expansion of $F(x, y)=I+\sum_{\substack{p=1 \\ m+n=p}}^{\infty} A_{m, n} x^{m} y^{n}$.

Definition 3.2.3. Given $F(x, y)=I+\sum_{\substack{p=1 \\ m+n=p}}^{\infty} A_{m, n} x^{m} y^{n}$, a formal power series with matrix coefficients or an analytic function of two independent complex variables with $F(0,0)=I$, we say $F(x, y)$ has a left to right (canonically ordered) matrix power product expansion in two independent variables if

$$
\begin{aligned}
& F(x, y)=\operatorname{LTR} \prod_{\substack{q=1 \\
m+n=q}}^{\infty}\left(I+G_{m, n} x^{m} y^{n}\right) \\
& =\left(I+G_{1,0} x^{1} y^{0}\right)\left(I+G_{0,1} x^{0} y^{1}\right)\left(I+G_{2,0} x^{2} y^{0}\right)\left(I+G_{1,1} x^{1} y^{1}\right)\left(I+G_{0,2} x^{0} y^{2}\right) \ldots
\end{aligned}
$$

where the ordering of the right side follows the conventions of Definition 1.2.1. We say $F(x, y)$ has a right to left (reversed canonically ordered) matrix power product expansion in two independent variables if

$$
\begin{aligned}
F(x, y) & =R T L \prod_{\substack{q=1 \\
m+n=q}}^{\infty}\left(I+G_{m, n} x^{m} y^{n}\right) \\
& =\ldots\left(I+G_{k_{1}, k_{2}} x^{k_{1}} y^{k_{2}}\right) \ldots\left(I+G_{2,0} x^{2} y^{0}\right)\left(I+G_{0,1} x^{0} y^{1}\right)\left(I+G_{1,0} x^{1} y^{0}\right)
\end{aligned}
$$


where the ordering of the right side follows the conventions of Definition 1.2.1 when read from right to left. We refer to the right side of either Equation (3.4) or (3.5) as an MPPE2. The expression $\left(I+G_{m, n} x^{m} y^{n}\right)$, where $(m, n) \in \mathbb{N}_{0} \times \mathbb{N}_{0}$, is an elementary factor of degree $m+n$. If $G_{m, n} \neq O$, the elementary factor is nontrivial. We make the convention of setting $G_{0,0}=I$.

We now define the notational conventions necessary for stating the formulas which algebraically represent the coefficients of the matrix power product expansion in terms of the power series coefficients. First off is a collection of vector indexed notations necessary for the statement of the structure property

Notation 3.2.1. The symbol $\phi=\left(\left(i_{1}, j_{1}\right),\left(i_{2}, j_{2}\right), \ldots,\left(i_{n}, j_{n}\right)\right)$ stands for a vector with $n$ components, where $n \in \mathbb{N}$ and $i_{1}, i_{2}, \ldots, i_{n}, j_{1}, j_{2}, \ldots, j_{n} \in \mathbb{N}_{0}$. Let $\tau=\tau(\phi)$ be the length of $\phi$, i.e. $\tau=n$. Let $|\phi|$ denote the sum of the components, i.e. $|\phi|=$ $\left(\sum_{d=1}^{n} i_{d}, \sum_{d=1}^{n} j_{d}\right)$. We denote $A_{\left(i_{k}, j_{k}\right), \phi}$ as $A_{\left(i_{k}, j_{k}\right),\left(i_{1}, j_{1}\right)} A_{\left(i_{k}, j_{k}\right),\left(i_{2}, j_{2}\right)} \ldots A_{\left(i_{k}, j_{k}\right),\left(i_{n}, j_{n}\right)}$.

Example 3.2.1. Let $\phi=((2,2),(1,3),(3,1),(2,2),(0,4),(4,0))$, then $\tau=\tau(\phi)=$ $6,|\phi|=(12,12)$, and

$$
A_{\left(i_{k}, j_{k}\right), \phi}=A_{\left(i_{k}, j_{k}\right),(2,2)} A_{\left(i_{k}, j_{k}\right),(1,3)} A_{\left(i_{k}, j_{k}\right),(3,1)} A_{\left(i_{k}, j_{k}\right),(2,2)} A_{\left(i_{k}, j_{k}\right),(0,4)} A_{\left(i_{k}, j_{k}\right),(4,0)} .
$$

Next, we define the notion of a suitable norm, a concept necessary for discussing the convergence of matrix power product expansions.

Definition 3.2.4. A norm $\|\cdot\|$ defined on the linear space of $d \times d$ matrices over $\mathbb{C}$ will be called absolute if

$$
\|A\|=\|(|A|)\| \text {. }
$$

It will be called monotonicity preserving if

$$
|A| \leq|B| \quad \text { implies that } \quad\|A\| \leq\|B\| \text {. }
$$

The norm will be called algebraic if

$$
\|A B\| \leq\|A\|\|B\|
$$

Finally, a norm $\|\cdot\|$ will be called suitable if it is absolute, monotonicity preserving, and algebraic. 


\subsection{Algebraic Formula for the Coefficients of a Ma- trix Power Product Expansion}

In this section and the next we study the expansion of a two variable power series into a MPPE2 and derive two algebraic representations for the coefficients $G_{m, n}$ as polynomials of the $\left(A_{m, n}\right)_{m, n}$. The first formula is almost an immediate consequence of Equation (3.4). Let

$$
F(x, y)=I+\sum_{\substack{p=1 \\ m+n=p}}^{\infty} A_{m, n} x^{m} y^{n}=L T R \prod_{\substack{q=1 \\ m+n=q}}^{\infty}\left(I+G_{m, n} x^{m} y^{n}\right) .
$$

By expanding the MPPE2 of Equation (3.6) into a formal power series, coefficient comparison shows that

$$
A_{m, n}=\sum_{\substack{i_{1}+i_{2}+\ldots+i_{r}=m \\ j_{1}+j_{2}+\ldots+j_{r}=n \\(1,0) \preceq\left(i_{1}, j_{1}\right) \prec\left(i_{2}, j_{2}\right) \prec \cdots \prec\left(i_{r}, j_{r}\right) \preceq(m, n)}} G_{i_{1}, j_{1}} G_{i_{2}, j_{2}} \ldots G_{i_{r}, j_{r}},
$$

or equivalently that

$$
G_{m, n}=A_{m, n}-\left(\sum_{\substack{i_{1}+i_{2}+\ldots+i_{r}=m \\ j_{1}+j_{2}+\ldots+j_{r}=n,(1,0) \preceq\left(i_{1}, j_{1}\right) \prec\left(i_{2}, j_{2}\right) \prec \cdots \prec\left(i_{r}, j_{r}\right) \prec(m, n), r \geq 2}} G_{i_{1}, j_{1}} G_{i_{2}, j_{2}} \ldots G_{i_{r}, j_{r}}\right),
$$

where the summation runs over all partitions of $(m, n)$ into distinct parts.

\subsection{Structure Property of the Coefficients of a Ma- trix Power Product Expansion}

There is another way to recursively express the coefficients $G_{m, n}$ as a multivariate polynomial of the variables $A_{m, n}$. The ultimate result of this methodology is the structure property, Theorem 3.4.2, a crucial result for determining convergence domains of power product expansions via a majorizing MPPE2. We begin with 
setting $A_{m, n}=A_{(1,0),(m, n)}$ and rewrite Equation (3.6) as

$$
\begin{aligned}
F(x, y) & =I+\sum_{\substack{p=1 \\
m+n=p}}^{\infty} A_{(1,0),(m, n)} x^{m} y^{n}=\left(I+G_{1,0} x\right)\left[L T R \prod_{\substack{q=1 \\
m+n=q \\
(m, n) \succeq(0,1)}}^{\infty}\left(I+G_{m, n} x^{m} y^{n}\right)\right] \\
& =\left(I+G_{1,0} x\right)\left[I+\sum_{\substack{p=1 \\
m+n=p \\
(m, n) \succeq(0,1)}}^{\infty} A_{(0,1),(m, n)} x^{m} y^{n}\right]
\end{aligned}
$$

where the summation conventions follow Definition 1.2.1.

Next we apply the same procedure to $I+\sum_{\substack{p=1 \\ m+n=p \\(m, n) \succeq(0,1)}}^{\infty} A_{(0,1),(m, n)} x^{m} y^{n}$ and find that

$$
I+\sum_{\substack{p=1 \\ m+n=p \\(m, n) \succeq(0,1)}}^{\infty} A_{(0,1),(m, n)} x^{m} y^{n}=I+A_{(0,1),(0,1)} y+A_{(0,1),(2,0)} x^{2}+A_{(1,0),(1,1)} x y+\ldots
$$

$$
\begin{gathered}
=\left(I+G_{0,1} y\right)\left[\operatorname{LTR} \prod_{\substack{q=2 \\
m+n=q \\
(m, n) \succeq(2,0)}}^{\infty}\left(I+G_{m, n} x^{m} y^{n}\right)\right] \\
=\left(I+G_{0,1} y\right)\left[I+\sum_{\substack{p=2 \\
m+n=p \\
(m, n) \succeq(2,0)}}^{\infty} A_{(2,0),(m, n)} x^{m} y^{n}\right] .
\end{gathered}
$$

By continuing this procedure inductively we define

$$
\left[I+\sum_{\substack{p=\ell \\ m+n=p \\(m, n) \succeq\left(i_{k}, j_{k}\right)}}^{\infty} A_{\left(i_{k}, j_{k}\right),(m, n)} x^{m} y^{n}\right]=\left(I+G_{i_{k}, j_{k}} x^{i_{k}} y^{j_{k}}\right)\left[I+\sum_{\substack{p=\ell \\ m+n=p \\(m, n) \succeq\left(i_{k+1}, j_{k+1}\right)}}^{\infty} A_{\left(i_{k+1}, j_{k+1}\right),(m, n)} x^{m} y^{n}\right]
$$

where $A_{\left(i_{k}, j_{k}\right),(0,0)}=I$ for all $(i, j) \in \mathbb{N}_{0} \times \mathbb{N}_{0}$, and $A_{\left(i_{k}, j_{k}\right),(m, n)}=O$, if $\left(i_{k}, j_{k}\right) \succ(m, n) \neq$ $(0,0)$. By comparing the coefficients of $x^{m} y^{n}$ in Equation (3.9), we find that

$$
A_{\left(i_{k+1}, j_{k+1}\right),(1,0)}=\cdots=A_{\left(i_{k+1}, j_{k+1}\right),\left(i_{k}, j_{k}\right)}=O,
$$




$$
A_{\left(i_{k+1}, j_{k+1}\right),(m, n)}=A_{\left(i_{k}, j_{k}\right),(m, n)}-G_{i_{k}, j_{k}} A_{\left(i_{k+1}, j_{k+1}\right),\left(m-i_{k}, n-j_{k}\right)} .
$$

If $(m, n)=\left(i_{k}, j_{k}\right)$, the final recurrence implies that

$$
G_{i_{k}, j_{k}}=A_{\left(i_{k}, j_{k}\right),\left(i_{k}, j_{k}\right)}
$$

Next use Equation (3.12) and the geometric series to rewrite Equation (3.9) as

$$
\begin{aligned}
& I+\sum_{\substack{p=\ell \\
m+n=p,(m, n) \succeq\left(i_{k+1}, j_{k+1}\right)}}^{\infty} A_{\left(i_{k+1}, j_{k+1}\right),(m, n)} x^{m} y^{n}=\left(I+G_{i_{k}, j_{k}} x^{i_{k}} y^{j_{k}}\right)^{-1}\left[I+\sum_{\substack{p=\ell \\
m+n=p \\
(m, n) \succeq\left(i_{k}, j_{k}\right)}}^{\infty} A_{\left(i_{k}, j_{k}\right),(m, n)} x^{m} y^{n}\right] \\
& =\left[I+\sum_{\alpha=1}^{\infty}(-1)^{\alpha}\left(G_{i_{k}, j_{k}} x^{i_{k}} y^{j_{k}}\right)^{\alpha}\right]\left[I+\sum_{\substack{p=\ell \\
m+n=p \\
(m, n) \succeq\left(i_{k}, j_{k}\right)}}^{\infty} A_{\left(i_{k}, j_{k}\right),(m, n)} x^{m} y^{n}\right] \\
& =\left[I+\sum_{\alpha=1}^{\infty}(-1)^{\alpha} A_{\left(i_{k}, j_{k}\right),\left(i_{k}, j_{k}\right)}^{\alpha}\left(x^{i_{k}} y^{j_{k}}\right)^{\alpha}\right]\left[I+\sum_{\substack{p=\ell \\
m+n=p \\
(m, n) \succeq\left(i_{k}, j_{k}\right)}}^{\infty} A_{\left(i_{k}, j_{k}\right),(m, n)} x^{m} y^{n}\right] .
\end{aligned}
$$

By equating the coefficient of $x^{s} y^{t}$ on both sides of Equation (3.13), we get

$$
A_{\left(i_{k+1}, j_{k+1}\right),(s, t)}=\sum_{\substack{\alpha i_{k}+m=s \\ \alpha j_{k}+n=t}}(-1)^{\alpha} A_{\left(i_{k}, j_{k}\right),\left(i_{k}, j_{k}\right)}^{\alpha} A_{\left(i_{k}, j_{k}\right),(m, n)} .
$$

Equation (3.14) is the key to proving the following theorem which is the precursor of the structure property.

Theorem 3.4.1. Let $\left(i_{k}, j_{k}\right) \in \mathbb{N}_{0} \times \mathbb{N}_{0} \backslash\{(0,0)\}$. Define $A_{\left(i_{k}, j_{k}\right),(0,0)}=I$ and $A_{\left(i_{k}, j_{k}\right),(m, n)}=$ O for $(1,0) \preceq(m, n) \preceq\left(i_{k-1}, j_{k-1}\right)$. Assume that $A_{\left(i_{k}, j_{k}\right),(m, n)} \leq$ Ofor all $\left(i_{k}, j_{k}\right) \preceq(m, n)$. Then $A_{\left(i_{k+1}, j_{k+1}\right),(s, t)} \leq O$ whenever $\left(i_{k+1}, j_{k+1}\right) \preceq(s, t)$.

Proof. Equation (3.14) is equivalent to

$$
\begin{aligned}
& A_{\left(i_{k+1}, j_{k+1}\right),(s, t)}=\sum_{\substack{\alpha i_{k}+m=s \\
\alpha j_{k}+n=t \\
(m, n) \neq(0,0),\left(i_{k}, j_{k}\right)}}(-1)^{\alpha} A_{\left(i_{k}, j_{k}\right),\left(i_{k}, j_{k}\right)}^{\alpha} A_{\left(i_{k}, j_{k}\right),(m, n)} \\
& +(-A)_{\left(i_{k}, j_{k}\right),\left(i_{k}, j_{k}\right)}^{\frac{s}{i_{k}}}+(-1)^{\frac{s}{i_{k}}-1}(A)_{\left(i_{k}, j_{k}\right),\left(i_{k}, j_{k}\right)}^{\frac{s}{i_{k}}},
\end{aligned}
$$


where the second term on the right side corresponds to $(m, n)=(0,0)$, while the third term corresponds to $(m, n)=\left(i_{k}, j_{k}\right)$. Rewrite Equation (3.15) as $A_{\left(i_{k+1}, j_{k+1}\right),(s, t)}=\beta+\gamma$, where

$$
\beta:=\sum_{\begin{array}{c}
\alpha i_{k}+m=s \\
\alpha j_{k}+n=t \\
(m, n) \neq(0,0),\left(i_{k}, j_{k}\right)
\end{array}}(-1)^{\alpha} A_{\left(i_{k}, j_{k}\right),\left(i_{k}, j_{k}\right)}^{\alpha} A_{\left(i_{k}, j_{k}\right),(m, n)},
$$

and

$$
\gamma:=(-A)_{\left(i_{k}, j_{k}\right),\left(i_{k}, j_{k}\right)}^{\frac{s}{i_{k}}}+(-1)^{\frac{s}{i_{k}}-1}(A)_{\left(i_{k}, j_{k}\right),\left(i_{k}, j_{k}\right)}^{\frac{s}{i_{k}}} .
$$

By the hypothesis, $A_{\left(i_{k}, j_{k}\right),\left(i_{k}, j_{k}\right)}^{\alpha} A_{\left(i_{k}, j_{k}\right),(m, n)}$ is the product of $\alpha+1$ non-positive matrices and is either a zero matrix or has a sign of $(-1)^{\alpha+1}$. Thus, $(-1)^{\alpha} A_{\left(i_{k}, j_{k}\right),\left(i_{k}, j_{k}\right)}^{\alpha} A_{\left(i_{k}, j_{k}\right),(m, n)}$ is either a zero or a negative matrix, and each summand in $\beta$ is a non-positive matrix.

It remains to show that $\gamma$ is also a non-positive matrix. Note that $\gamma$ only exists if $\alpha=\frac{s}{i_{k}}$ is a positive integer, say $\frac{s}{i_{k}}=\widehat{\alpha}>1$, in which case $\gamma$ becomes

$$
\gamma=(-A)_{\left(i_{k}, j_{k}\right),\left(i_{k}, j_{k}\right)}^{\widehat{\alpha}}+(-1)^{\widehat{\alpha}-1}(A)_{\left(i_{k}, j_{k}\right),\left(i_{k}, j_{k}\right)}^{\widehat{\alpha}}=O .
$$

Therefore, the representation of $\gamma$ provided by Equation (3.16) shows that $\gamma$ is a nonpositive matrix.

Theorem 3.4.2. (Structure Property) Let $\left(i_{k}, j_{k}\right) \in \mathbb{N}_{0} \times \mathbb{N}_{0} \backslash\{(0,0)\}$. Then

$$
A_{\left(i_{k+1}, j_{k+1}\right),(s, t)}=\sum_{\eta}(-1)^{\tau(\phi(\eta))-1} A_{\left(i_{k}, j_{k}\right), \phi(\eta)}=\sum_{\eta}(-1)^{\tau(\phi(\eta))+1} A_{\left(i_{k}, j_{k}\right), \phi(\eta)},
$$

where the sum is over all $\phi(\eta)=\left(\left(i_{1}, j_{1}\right),\left(i_{2}, j_{2}\right), \ldots,\left(i_{\tau}, j_{\tau}\right)\right)$ such that $|\phi(\eta)|=(s, t)$ and $\left(i_{p}, j_{p}\right)=\left(i_{k}, j_{k}\right)$ whenever $1 \leq p \leq \tau-1$. (Recall that we defined $A_{\left(i_{k}, j_{k}\right), \phi(\eta)}=$ $\left.A_{\left(i_{k}, j_{k}\right),\left(i_{1}, j_{1}\right)} \ldots A_{\left(i_{k}, j_{k}\right),\left(i_{\tau}, j_{\tau}\right)}.\right)$ If $A_{\left(i_{k}, j_{k}\right),(s, t)} \leq O$ and all $\left(i_{k}, j_{k}\right) \preceq(s, t)$, then Equation (3.17) is equivalent to

$$
A_{\left(i_{k+1}, j_{k+1}\right),(s, t)}=-\sum_{\eta}\left|A_{\left(i_{k}, j_{k}\right),\left(i_{1}, j_{1}\right)}\right| \ldots\left|A_{\left(i_{k}, j_{k}\right),\left(i_{\tau}, j_{\tau}\right)}\right|,
$$

where the range of summation is identical to the range of summation used in Equation (3.17).

Proof. We obtain the desired result by representing $A_{\left(i_{k}, j_{k}\right),\left(i_{k}, j_{k}\right)}^{\alpha} A_{\left(i_{k}, j_{k}\right),(m, n)}$ in Equation (3.14) as $A_{\left(i_{k}, j_{k}\right), \phi(\eta)}$ and then applying Theorem 3.4.1.

In order to obtain a formulation of the structure property that is useful for determining the domain of convergence for an MPPE2, we need to iterate Equation (3.18). Let 
$\phi=\left(\left(i_{1}, j_{1}\right),\left(i_{2}, j_{2}\right), \ldots,\left(i_{n}, j_{n}\right)\right)$ denote a vector with $n$ components, where $n \in \mathbb{N}$, and $i_{1}, i_{2}, \ldots, i_{n}, j_{1}, j_{2}, \ldots, j_{n} \in \mathbb{N}_{0}$, and let $A_{\phi(\eta)}$ denote the expression $A_{i_{1}, j_{1}} A_{i_{2}, j_{2}} \ldots A_{i_{n}, j_{n}}$. After $L$ iterations, and assuming $A_{\left(i_{k}, j_{k}\right),(s, t)} \leq O$, whenever $\left(i_{k}, j_{k}\right) \preceq(s, t)$, we obtain

$$
A_{\left(i_{k+1}, j_{k+1}\right),(s, t)}=\sum_{\eta}(-1)^{\tau(\phi(\eta))-1} A_{\phi(\eta)}=-\sum_{\eta}\left|A_{i_{1}, j_{1}}\right|\left|A_{i_{2}, j_{2}}\right| \ldots\left|A_{i_{\tau}, j_{\tau}}\right|,
$$

where the sum is over $\phi(\eta)=\left(\left(i_{1}, j_{1}\right),\left(i_{2}, j_{2}\right), \ldots,\left(i_{\tau}, j_{\tau}\right)\right)$ such that $|\phi(\eta)|=(s, t)$.

$$
\text { If }(s, t)=\left(i_{k+1}, j_{k+1}\right) \text {, then Equation (3.19) becomes }
$$

$$
\begin{aligned}
A_{\left(i_{k+1}, j_{k+1}\right),\left(i_{k+1}, j_{k+1}\right)}=G_{i_{k+1}, j_{k+1}} & =\sum_{\eta}(-1)^{\tau(\phi(\eta))-1} A_{\phi(\eta)} \\
& =-\sum_{\eta}\left|A_{i_{1}, j_{1}}\right|\left|A_{i_{2}, j_{2}}\right| \ldots\left|A_{i_{\tau}, j_{\tau}}\right|,
\end{aligned}
$$

where the sum is over $\phi(\eta)=\left(\left(i_{1}, j_{1}\right),\left(i_{2}, j_{2}\right), \ldots,\left(i_{\tau}, j_{\tau}\right)\right)$ such that $|\phi(\eta)|=\left(i_{k+1}, j_{k+1}\right)$. To illustrate application the structure property, we furnish the following example:

$$
\begin{array}{rlr}
G_{2,1}= & A_{(2,1),(2,1)} & \text { By using Equat } \\
= & A_{(3,0),(2,1)} & \text { By using Equ } \\
= & A_{(0,2),(2,1)} \\
= & A_{(1,1),(2,1)} \\
= & A_{(2,0),(2,1)} \\
= & (-1)^{1} A_{(0,1),(0,1)} A_{(0,1),(2,0)}+A_{(0,1),(2,1)} \\
= & (-1)^{1} A_{(1,0),(0,1)} A_{(1,0),(2,0)}+(-1)^{1} A_{(1,0),(1,0)} A_{(1,0),(1,1)}+ \\
& (-1)^{2} A_{(1,0),(1,0)}^{2} A_{(1,0),(0,1)}+A_{(1,0),(2,1)}
\end{array}
$$

By using Equation (3.12)

By using Equation (3.14)

The explicit coefficients $G_{m, n}$ with $1 \leq m+n \leq 4$, as polynomials of $A_{1,0}, \ldots, A_{m, n}$, are given below.

$$
\begin{aligned}
G_{1,0} & =(-1)^{0} A_{1,0} \\
G_{0,1} & =(-1)^{0} A_{0,1} \\
G_{2,0} & =(-1)^{0} A_{2,0} \\
G_{1,1} & =(-1)^{0} A_{1,1}+(-1)^{1} A_{1,0} A_{0,1} \\
G_{0,2} & =(-1)^{0} A_{0,2} \\
G_{3,0} & =(-1)^{0} A_{3,0}+(-1)^{1} A_{1,0} A_{2,0}
\end{aligned}
$$




$$
\begin{aligned}
G_{2,1}= & (-1)^{0} A_{2,1}+(-1)^{1} A_{0,1} A_{2,0}+(-1)^{1} A_{1,0} A_{1,1}+(-1)^{2} A_{1,0}^{2} A_{0,1} \\
G_{1,2}= & (-1)^{0} A_{1,2}+(-1)^{1} A_{1,0} A_{0,2}+(-1)^{1} A_{0,1} A_{1,1}+(-1)^{2} A_{0,1} A_{1,0} A_{0,1} \\
G_{0,3}= & (-1)^{0} A_{0,3}+(-1)^{1} A_{0,1} A_{0,2} \\
G_{4,0}= & (-1)^{0} A_{4,0}+(-1)^{1} A_{1,0} A_{3,0}+(-1)^{2} A_{1,0}^{2} A_{2,0} \\
G_{3,1}= & (-1)^{0} A_{3,1}+(-1)^{1} A_{0,1} A_{3,0}+(-1)^{2} A_{0,1} A_{1,0} A_{2,0}+(-1)^{1} A_{1,0} A_{2,1}+ \\
& (-1)^{2} A_{1,0}^{2} A_{1,1}+(-1)^{3} A_{1,0}^{3} A_{0,1}+(-1)^{1} A_{2,0} A_{1,1}+(-1)^{2} A_{2,0} A_{1,0} A_{0,1} \\
G_{2,2}= & (-1)^{0} A_{2,2}+(-1)^{1} A_{2,0} A_{0,2}+(-1)^{1} A_{1,0} A_{1,2}+(-1)^{2} A_{1,0} A_{0,1} A_{1,1}+ \\
& A_{1,0}^{2} A_{0,2}+(-1)^{1} A_{0,1} A_{2,1}+(-1)^{2} A_{0,1}^{2} A_{2,0}+(-1)^{3} A_{0,1} A_{1,0}^{2} A_{0,1} \\
G_{1,3}= & (-1)^{0} A_{1,3}+(-1)^{1} A_{1,0} A_{0,3}+(-1)^{1} A_{0,1} A_{2,1}+(-1)^{2} A_{0,1}^{2} A_{1,1}+ \\
& (-1)^{2} A_{0,1} A_{1,0} A_{0,2}+(-1)^{3} A_{0,1}^{2} A_{1,0} A_{0,1}+(-1)^{1} A_{1,1} A_{0,2}+ \\
& (-1)^{2} A_{1,0} A_{0,1} A_{0,2} \\
G_{0,4}= & (-1)^{0} A_{0,4}+(-1)^{1} A_{0,1} A_{0,3}+(-1)^{2} A_{0,1}^{2} A_{0,2} .
\end{aligned}
$$

An examination of $G_{3,1}$ brings to the fore the pronounced difference between the scalar (commutative) case treated in chapter one and the current non-commutative case since two terms $A_{0,1} A_{1,0} A_{2,0}$ and $A_{2,0} A_{1,0} A_{0,1}$ can't combine together. This lack of commutativity implies that in the non-commutative case $G_{m, n}$ possess a finer decomposition of structured terms than what was found in the scalar case.

\subsection{Convergence Criteria for MPPE2's}

The purpose of this section is to present results concerning the domain of convergence of the MPPE2 in Equation (3.4). Since we are dealing with doubly indexed infinite products, we must carefully define what is meant by the convergence of $\operatorname{LTR} \underset{\substack{q=1 \\ m+n=q}}{\infty}\left(I+G_{m, n} x^{m} y^{n}\right)$.

Definition 3.5.1. An MPPE2 LTR $\prod_{\substack{q=1 \\ m+n=q}}^{\infty}\left(I+G_{m, n} x^{m} y^{n}\right)$ converges if and only if

$\lim _{P \rightarrow \infty} L T R \prod_{\substack{q=1 \\ m+n=q}}^{P}\left(I+G_{m, n} x^{m} y^{n}\right)$ converges to a nonzero complex number. Note that the order of the elementary factors within LTR $\prod_{\substack{q=1 \\ m+n=q}}^{P}\left(I+G_{m, n} x^{m} y^{n}\right)$ follows the conventions of Definition 1.2.1. 
We are primarily interested in the absolute convergence of $\operatorname{LTR} \prod_{\substack{q=1 \\ m+n=q}}^{\infty}\left(I+G_{m, n} x^{m} y^{n}\right)$. Therefore, following the lead of J. Thunder [43], we make the following definition.

Definition 3.5.2. For a suitable norm the MPPE2LTR $\prod_{\substack{q=1 \\ m+n=q}}^{\infty}\left(I+G_{m, n} x^{m} y^{n}\right)$ is absolutely convergent if and only if $\prod_{\substack{q=1 \\ m+n=q}}^{\infty}\left(1+|| G_{m, n}||\left|x^{m} y^{n}\right|\right)$ converges, that is if and only if $\lim _{m, n \rightarrow \infty} \prod_{i=1}^{n} \prod_{j=1}^{m}\left(1+|| G_{i, j}||\left|x^{i} y^{j}\right|\right)$ converges to a nonzero real number.

Since $e^{x} \geq x+1$ whenever $x \geq 0$, we observe that

$$
\begin{aligned}
\sum_{i=1}^{m} \sum_{j=1}^{n} \| G_{i, j}||\left|x^{i} y^{j}\right| & <\prod_{i=1}^{m} \prod_{j=1}^{n}\left(1+\| G_{i, j}||\left|x^{i} y^{j}\right|\right) \\
& \leq \prod_{i=1}^{m} \prod_{j=1}^{n} e^{\| G_{i, j}|| x^{i} y^{j} \mid}=e^{\sum_{i=1}^{m} \sum_{j=1}^{n}|| G_{i, j}|| x^{i} y^{j} \mid} .
\end{aligned}
$$

Thus Lemma 2 of [43] is applicable and we have the following proposition:

Proposition 3.5.1. Given a suitable matrix norm, an MPPE2 $\operatorname{LTR} \prod_{\substack{q=1 \\ m+n=q}}^{\infty}\left(I+G_{m, n} x^{m} y^{n}\right)$ is absolutely convergent if and only if $\sum_{\substack{q=1 \\ m+n=q}}^{\infty}|| G_{m, n}||\left|x^{m} y^{n}\right|$ is an absolutely convergent series of real numbers.

As it the case of a double series, if an MPPE2 $\operatorname{LTR} \prod_{\substack{q=1 \\ m+n=q}}^{\infty}\left(I+G_{m, n} x^{m} y^{n}\right)$ is absolutely convergent, then order of multiplication is immaterial and thus we choose to apply the ordering of Definition 1.2.1. We implicitly made use of this fact in Proposition 3.5.1.

If the coefficients $\left(G_{m, n}\right)$ of the MPPE2 are commutative more can be said in terms of the power series expansion of $\log \left(I+G_{m, n} x^{m} y^{n}\right)$. For any two commutative matrices $A, B \in M_{d}(\mathbb{C})$, it can be shown that $e^{A+B}=e^{A} e^{B}$ [[14], Proposition 2.5, P. 35]. Thus, if $\left(G_{m, n}\right)$ is a sequence of commutative matrices matrices, we define

$$
\log \left(\operatorname{LTR} \prod_{\substack{q=1 \\ m+n=q}}^{\infty}\left(I+G_{m, n} x^{m} y^{n}\right)\right):=\sum_{\substack{q=1 \\ m+n=q}}^{\infty} \log \left(I+G_{m, n} x^{m} y^{n}\right),
$$

where

$$
\log \left(I+G_{m, n} x^{m} y^{n}\right):=\sum_{\ell=1}^{\infty}(-1)^{\ell+1} \frac{G_{m, n}^{\ell}}{\ell} x^{m \ell} y^{n \ell} .
$$


Equation (3.21) implies that

$$
\exp \left(\sum_{\substack{q=1 \\ m+n=q}}^{\infty} \log \left(I+G_{m, n} x^{m} y^{n}\right)\right)=\operatorname{LTR} \prod_{\substack{q=1 \\ m+n=q}}^{\infty}\left(I+G_{m, n} x^{m} y^{n}\right),
$$

and implies that for a sequence of commutative coefficients $\left(G_{m, n}\right)$, the MPPE2 $\underset{\substack{q=1 \\ m+n=q}}{\infty}\left(I+G_{m, n} x^{m} y^{n}\right)$ converges to a nonzero value if and only if the double series

$$
\sum_{\substack{q=1 \\ m+n=q}}^{\infty} \log \left(I+G_{m, n} x^{m} y^{n}\right)=\sum_{\substack{q=1 \\ m+n=q}}^{\infty} \sum_{\ell=1}^{\infty}(-1)^{\ell+1} \frac{G_{m, n}^{\ell}}{\ell} x^{m \ell} y^{n \ell}
$$

is convergent.

By adapting the Taylor series argument found on Page 165 of [5] we have

$$
1 / 2\|\Delta\| \leq\|\log (I+\Delta)\| \leq 3 / 2\|\Delta\|, \quad\|\Delta\|<1 / 2 .
$$

(In our case $\Delta=G_{m, n} x^{m} y^{n}$.) Equation (3.24) implies that $\sum_{\substack{q=1 \\ m+n=q}}^{\infty} \log \left(I+G_{m, n} x^{m} y^{n}\right)$ is absolutely convergent if and only if $\sum_{\substack{q=1 \\ m+n=q}}^{\infty} G_{m, n} x^{m} y^{n}$ is absolutely convergent. We summarize the previous discussion in the follow proposition:

Proposition 3.5.2. Let $\left(G_{m, n}\right)$ be a sequence of commutative matrices associated with the matrix function $F(x, y)=\operatorname{LTR} \prod_{\substack{q=1 \\ m+n=q}}^{\infty}\left(I+G_{m, n} x^{m} y^{n}\right)$. Define

$$
\log F(x, y):=\sum_{\substack{q=1 \\ m+n=q}}^{\infty} \log \left(I+G_{m, n} x^{m} y^{n}\right)=\sum_{\substack{q=1 \\ m+n=q}}^{\infty} \sum_{\ell=1}^{\infty}(-1)^{\ell+1} \frac{G_{m, n}^{\ell}}{\ell} x^{m \ell} y^{n \ell} .
$$

The MPEE2 of $F(x, y), \operatorname{LTR} \prod_{\substack{q=1 \\ m+n=q}}^{\infty}\left(I+G_{m, n} x^{m} y^{n}\right)$, converges to a nonzero value if and only $\log F(x, y)=\sum_{\substack{q=1 \\ m+n=q}}^{\infty} \log \left(I+G_{m, n} x^{m} y^{n}\right)$ converges. The MPEE2 of $F(x, y)$, LTR $\prod_{\substack{q=1 \\ m+n=q}}^{\infty}(I+$ $\left.G_{m, n} x^{m} y^{n}\right)$, is absolutely convergent if and only if $\sum_{\substack{q=1 \\ m+n=q}}^{\infty} \log \left(I+G_{m, n} x^{m} y^{n}\right)$ is absolutely convergent if and only if $\sum_{\substack{q=1 \\ m+n=q}}^{\infty}\left\|\sum_{\ell=1}^{\infty} \frac{(-1)^{\ell+1} G_{m, n}^{\ell}}{\ell} x^{m \ell} y^{n \ell}\right\|<\infty$. 
The first main result of this section provides a lower bound for the domain of convergence an MPPE2 of in terms a majorizing log series.

Theorem 3.5.1. (1.) Given a matrix function $F(x, y)$

$$
F(x, y)=I+\sum_{\substack{p=1 \\ m+n=p}}^{\infty} A_{m, n} x^{m} y^{n}=L T R \prod_{\substack{q=1 \\ m+n=q}}^{\infty}\left(I+G_{m, n} x^{m} y^{n}\right),
$$

where $A_{m, n}, G_{m, n} \in M_{d}(\mathbb{C})$, define the following auxiliary matrices functions with coefficients over $M_{d}(\mathbb{R})$ :

$$
\begin{aligned}
& C(x, y)=I-\sum_{\substack{p=1 \\
m+n=p}}^{\infty}\left|A_{m, n}\right| x^{m} y^{n}=L T R \prod_{\substack{q=1 \\
m+n=q}}^{\infty}\left(I-C_{m, n} x^{m} y^{n}\right) \\
& M(x, y)=I-\sum_{\substack{p=1 \\
m+n=p}}^{\infty} M_{m, n} x^{m} y^{n}=L T R \prod_{\substack{q=1 \\
m+n=q}}^{\infty}\left(I-E_{m, n} x^{m} y^{n}\right) .
\end{aligned}
$$

If $\left|A_{m, n}\right| \leq M_{m, n}$ for all $(m, n) \in \mathbb{N}_{0} \times \mathbb{N}_{0} \backslash\{(0,0)\}$, then

$$
O \leq\left|G_{m, n}\right| \leq C_{m, n} \leq E_{m, n} \quad \text { for all } \quad(m, n) \in \mathbb{N}_{0} \times \mathbb{N}_{0} \backslash\{(0,0)\} .
$$

(2.) Define $W \in M_{d}(\mathbb{C})$ as

$$
W=\left(w_{u, v}\right), \quad \text { where } \quad w_{u, v}=1, \quad v, u=1, \ldots, d .
$$

Furthermore, given $A_{m, n} \in M_{d}(\mathbb{C})$ where $A_{m, n}=\left(a_{\psi, \omega}(m, n)\right)$ for $\psi, \omega=1, \ldots, d$, define

$$
a(m, n):=\max _{\psi, \omega}\left|a_{\psi, \omega}(m, n)\right|, \quad \text { and } \quad s:=\sup _{m+n \geq 1}[a(m, n)]^{\frac{1}{m+n}} .
$$

Consider a special case for $M(x, y)$, namely

$$
M(x, y)=I-\sum_{\substack{p=1 \\ m+n=p}}^{\infty} s^{m+n} W x^{m} y^{n}=L T R \prod_{\substack{q=1 \\ m+n=q}}^{\infty}\left(I-E_{m, n} x^{m} y^{n}\right),
$$

where $M_{m, n}=s^{m+n} W$. Since $\left(s^{m+n} W\right)$ is a sequence of commutative matrices, $\left(E_{m, n}\right)$ is also a sequence of commutative matrices. By using the Taylor series 
of expansion $\log \left(I-E_{m, n} x^{m} y^{n}\right)$, we define

$$
\begin{aligned}
\log M(x, y) & =\log \left(\operatorname{LTR} \prod_{\substack{q=1 \\
m+n=q}}^{\infty}\left(I-E_{m, n} x^{m} y^{n}\right)\right) \\
& =\sum_{\substack{q=1 \\
m+n=q}}^{\infty} \log \left(I-E_{m, n} x^{m} y^{n}\right)=-\sum_{\substack{q=1 \\
m+n=q}}^{\infty} \sum_{\ell=1}^{\infty} \frac{E_{m, n}^{\ell}\left(x^{m} y^{n}\right)^{\ell}}{\ell} \\
& =\sum_{\substack{p=1 \\
m+n=p}}^{\infty} P_{m, n} x^{m} y^{n}, \quad P_{m, n}:=-\sum_{\substack{\ell \mid d \\
d=g c d(m, n)}} \frac{\left(E_{\frac{m}{\ell}, \frac{n}{\ell}}^{\ell}\right.}{\ell} .
\end{aligned}
$$

Let $\|\cdot\|$ be a suitable norm and let $\rho(\|\cdot\|)$ be the radius of convergence of

$$
\begin{aligned}
& \sum_{\substack{p=1 \\
m+n=p}}^{\infty} P_{m, n} x^{m} y^{n} \text { as defined via Equation (3.29). Then } \\
& \qquad O \leq\left|G_{m, n}\right| \leq C_{m, n} \leq E_{m, n} \leq-P_{m, n}, \quad(m, n) \in \mathbb{N}_{0} \times \mathbb{N}_{0} .
\end{aligned}
$$

The inequalities of (3.30), when combined with Proposition 3.5.1, imply that LTR $\prod_{\substack{q=1 \\ m+n=q}}^{\infty}\left(I+G_{m, n} x^{m} y^{n}\right)$ converges absolutely for $\rho(\|\cdot\|)$.

Proof. (1) Equation (3.20) implies that

$$
\begin{gathered}
G_{m, n}=\sum_{\eta}(-1)^{\tau(\phi(\eta))+1} A_{\phi(\eta)}=\sum_{\eta}(-1)^{\tau(\phi(\eta))+1} A_{\left(i_{1}, j_{1}\right)} \ldots A_{\left(i_{\tau}, j_{\tau}\right)}, \\
|\phi(\eta)|=(m, n)
\end{gathered}
$$

which in turn implies that

$$
\left|G_{m, n}\right|=\left|\sum_{\substack{\eta \\|\phi(\eta)|=(m, n)}}(-1)^{\tau(\phi(\eta))+1} A_{i_{1}, j_{1}} \ldots A_{i_{\tau}, j_{\tau}}\right| \leq \sum_{\substack{\eta \\|\phi(\eta)|=(m, n)}}\left|A_{i_{1}, j_{1}}\right| \ldots\left|A_{i_{\tau}, j_{\tau}}\right| .
$$

Similarly, when we apply Equation (3.20) to Equation (3.26), we obtain

$$
\begin{aligned}
O \leq C_{m, n} & =\sum_{\eta}(-1)^{\tau(\phi(\eta))}\left(-\left|A_{i_{1}, j_{1}}\right|\right) \ldots\left(-\left|A_{i_{\tau}, j_{\tau}}\right|\right) \\
& =\sum_{\eta}(-1)^{\tau(2 \phi(\eta), n)}\left(\left|A_{i_{1}, j_{1}}\right|\right) \ldots\left(\left|A_{i_{\tau}, j_{\tau}}\right|\right) \\
& =\sum_{\substack{\eta \\
|\phi(\eta)|=(m, n)}}\left(\left|A_{i_{1}, j_{1}}\right|\right) \ldots\left(\left|A_{i_{\tau}, j_{\tau}}\right|\right) .
\end{aligned}
$$


Combining Equation (3.31) and (3.32), we deduce that $\left|G_{m, n}\right| \leq C_{m, n}$. Also due to the inequality $\left|A_{m, n}\right| \leq M_{m, n}$, we have

$$
O \leq C_{m, n}=\sum_{\substack{\eta \\|\phi(\eta)|=(m, n)}}\left|A_{i_{1}, j_{1}}\right| \ldots\left|A_{i_{\tau}, j_{\tau}}\right| \leq \sum_{\substack{\eta \\|\phi(\eta)|=(m, n)}} M_{i_{1}, j_{1}} \ldots M_{i_{\tau}, j_{\tau}}=E_{m, n},
$$

and hence $0 \leq\left|G_{m, n}\right| \leq C_{m, n} \leq E_{m, n}$.

(2) By the definition of $P_{m, n}$ provided via Equation (3.29), we see that

$$
-P_{m, n}=\sum_{\substack{\ell \mid d \\
d=\operatorname{gcd}(m, n)}} \frac{\left(E_{\frac{m}{\ell}, \frac{n}{\ell}}\right)^{\ell}}{\ell}=E_{m, n}+\sum_{\begin{array}{c}
\ell \mid d, \ell \neq 1 \\
d=\operatorname{gcd}(m, n)
\end{array}} \frac{\left(E_{\frac{m}{\ell}, \frac{n}{\ell}}\right)^{\ell}}{\ell}
$$

and since Part (1) implies that for an arbitrary pair of subscripts $(\alpha, \beta) E_{\alpha, \beta} \geq O$, the preceding equation implies that

$$
O \leq E_{m, n} \leq-P_{m, n}
$$

which completes the proof of (3.30).

Let us take a moment to reestimate the power series expansion of $\log M(x, y)=\sum_{\substack{p=1 \\ m+n=p}}^{\infty} P_{m, n} x^{m} y^{n}$.

Since $W^{n}=d^{n-1} W$, we find that

$$
\begin{aligned}
-\log M(x, y) & =-\log \left[I-W \sum_{\substack{m+n=p \\
m}}^{\infty} s^{m+n} x^{m} y^{n}\right] \\
& =-\log \left[I-W\left[\sum_{m=0}^{\infty}(s x)^{m} \sum_{n=0}^{\infty}(s y)^{n}-1\right]\right] \\
& =-\log \left[I-W\left[\frac{1}{1-s x} \cdot \frac{1}{1-s y}-1\right]\right] \\
& =-\log \left[I-W\left[\frac{s(x+y)-s^{2} x y}{(1-s x)(1-s y)}\right]\right] \\
& =\sum_{p=1}^{\infty} \frac{W^{p}}{p}\left[\frac{s(x+y)-s^{2} x y}{(1-s x)(1-s y)}\right]^{p} \\
& \leq \sum_{p=1}^{\infty} W^{p}\left[\frac{s(x+y)-s^{2} x y}{(1-s x)(1-s y)}\right]^{p} \\
& =\frac{W}{d} \sum_{p=1}^{\infty}\left[\frac{d s(x+y)-d s^{2} x y}{(1-s x)(1-s y)}\right]^{p}
\end{aligned}
$$




$$
\begin{aligned}
& \leq \frac{W}{d} \sum_{p=0}^{\infty}\left[\frac{d s(x+y)-d s^{2} x y}{(1-s x)(1-s y)}\right]^{p} \\
& =\frac{W}{d} \frac{1}{1-\frac{d s(x+y)-d s^{2} x y}{(1-s x)(1-s y)}}, \quad \text { where }\left|\frac{d s(x+y)-d s^{2} x y}{(1-s x)(1-s y)}\right|<1 \\
& =\frac{W}{d} \frac{(1-s x)(1-s y)}{1-(d+1) s(x+y)+(d+1) s^{2} x y} .
\end{aligned}
$$

The preceding calculation implies that an estimate for the domain of convergence is related to the domain of convergence of scalar function $\phi^{-1}(x, y)=\frac{(1-s x)(1-s y)}{1-(d+1) s(x+y)+(d+1) s^{2} x y}$. By applying an appropriate similarity transformation, $\phi(x, y)$ appears in the expansion of $M(x, y)=I-W \sum_{\substack{p=1 \\ m+n=p}}^{\infty} s^{m+n} x^{m} y^{n}$. Since $W^{T}=W$ and $|\lambda I-W|=\lambda^{d-1}(\lambda-d)$, the eigenvalues of $W$ are $\lambda_{1}=d, \lambda_{2}=\lambda_{3}=\cdots=\lambda_{d}=0$, and there exists an orthogonal matrix $Q$ such that $Q^{T}=Q^{-1}[[15]$, Theorem 16.19, P. 571] with

$$
W=Q\left(\begin{array}{cccc}
d & 0 & \cdots & 0 \\
0 & 0 & 0 & \vdots \\
\vdots & 0 & \ddots & 0 \\
0 & \cdots & 0 & 0
\end{array}\right) Q^{T}
$$

The left hand side of Equation (3.28) becomes

$$
\begin{aligned}
M(x, y) & =I-\sum_{\substack{p=1 \\
m+n=p}}^{\infty} s^{m+n} W x^{m} y^{n} \\
& =I-\sum_{\substack{p=1 \\
m+n=p}}^{\infty}\left(\begin{array}{rrrr}
d & 0 & \cdots & 0 \\
0 & 0 & 0 & \vdots \\
\vdots & 0 & \ddots & 0 \\
0 & \cdots & 0 & 0
\end{array}\right) Q^{T} s^{m+n} x^{m} y^{n} \\
& =Q\left[\begin{array}{cccc}
d & 0 & \cdots & 0 \\
\left.I-\sum_{\substack{p=1 \\
m+n=p}}^{\infty}\left(\begin{array}{rrrr}
0 & 0 & 0 & \vdots \\
\vdots & 0 & \ddots & 0 \\
0 & \cdots & 0 & 0
\end{array}\right) s^{m+n} x^{m} y^{n}\right] Q^{T}
\end{array}\right]
\end{aligned}
$$




$$
\begin{aligned}
& =Q\left[I-\left(\begin{array}{cccc}
d \sum_{\substack{p=1 \\
m+n=p}}^{\infty} s^{m+n} x^{m} y^{n} & 0 & \cdots & 0 \\
0 & 0 & 0 & \vdots \\
\vdots & 0 & \ddots & 0 \\
0 & 0 & \cdots & 0
\end{array}\right)\right] Q^{T} \\
& =Q\left(\begin{array}{cccc}
\phi(x, y) & 0 & \cdots & 0 \\
0 & 1 & 0 & \vdots \\
\vdots & 0 & \ddots & 0 \\
0 & 0 & \cdots & 1
\end{array}\right) Q^{T} \text {, }
\end{aligned}
$$

where

$$
\begin{aligned}
\phi(x, y) & =1-d \sum_{\substack{p=1 \\
m+n=p}}^{\infty} s^{m+n} x^{m} y^{n}=1-d\left(\sum_{m=0}^{\infty}(s x)^{m} \sum_{n=0}^{\infty}(s y)^{n}-1\right) \\
& =1-d\left(\frac{1}{1-s x} \cdot \frac{1}{1-s y}-1\right)=\frac{1-s(d+1)(x+y)+s^{2}(d+1) x y}{(1-s x)(1-s y)} .
\end{aligned}
$$

Since we are ultimately computing $\log (\phi(x, y))$, we set the domain of definition for $\phi(x, y)$ to be $C^{\infty}\left(\mathbb{C}^{2} \backslash S\right)$, where

$$
S=\left\{x=\frac{1}{s}, y=\frac{1}{s}, \text { and } s(d+1)(x+y)+s^{2}(d+1) x y=1\right\} .
$$

Next we check for which values $(x, y)$ the series $\log (M(x, y))=\sum_{\substack{p=1 \\ m+n=p}}^{\infty} P_{m, n} x^{m} y^{n}$ as defined in Equation (3.29) will be absolutely convergent. By taking the logarithm of Equation (3.33), we have

$$
\begin{aligned}
\log (M(x, y)) & =\log \left[I-\sum_{\substack{p=1 \\
m+n=p}}^{\infty} s^{m+n} W x^{m} y^{n}\right] \\
& =\log \left[Q\left(\begin{array}{cccc}
\phi(x, y) & 0 & \cdots & 0 \\
0 & 1 & 0 & \vdots \\
\vdots & 0 & \ddots & 0 \\
0 & \cdots & 0 & 1
\end{array}\right) Q^{T}\right]
\end{aligned}
$$




$$
=Q\left(\begin{array}{cccc}
\log [\phi(x, y)] & 0 & \cdots & 0 \\
0 & \log 1 & 0 & \vdots \\
\vdots & 0 & \ddots & 0 \\
0 & 0 & \cdots & \log 1
\end{array}\right) Q^{T}
$$

where the last equality made use of the fact that $e^{U A U^{-1}}=U e^{A} U^{-1}$ whenever $A, U \in$ $M_{d}(\mathbb{C})$ [[14], Prop. 2.2, Page 33]. Therefore, it suffices to consider where the function $\log [\phi(x, y)]$ will be absolutely convergent. Observe that

$$
\begin{aligned}
\log [\phi(x, y)] & =\log \left[1-d \sum_{\substack{p=1 \\
m+n=p}}^{\infty} s^{m+n} x^{m} y^{n}\right] \\
& =\log \left[\frac{1-s(d+1)(x+y)+s^{2}(d+1) x y}{(1-s x)(1-s y)}\right] \\
& =\log \left(1-\left[s(d+1)(x+y)-s^{2}(d+1) x y\right]\right)-\log (1-s x)-\log (1-s y) \\
& =\sum_{\ell=1}^{\infty} \frac{(s x)^{\ell}}{\ell}+\sum_{\ell=1}^{\infty} \frac{(s y)^{\ell}}{\ell}-\sum_{\ell=1}^{\infty} \frac{\left[s(d+1)(x+y)-s^{2}(d+1) x y\right]^{\ell}}{\ell} .
\end{aligned}
$$

The three series in (3.35) are absolutely convergent for $|x|<\frac{1}{s},|y|<\frac{1}{s}$, and for $\left|s(d+1)(x+y)-s^{2}(d+1) x y\right|<1$, respectively. By the triangle inequality, we have

$$
\left|s(d+1)(x+y)-s^{2}(d+1) x y\right| \leq s(d+1)(|x|+|y|)+s^{2}(d+1)|x||y| .
$$

If we require $s(d+1)(|x|+|y|)+s^{2}(d+1)|x||y|<1$, since

$$
s(d+1)|x|<s(d+1)(|x|+|y|)+s^{2}(d+1)|x||y|<1,
$$

we find that $|x|<\frac{1}{s(d+1)}$. Similarly, $|y|<\frac{1}{s(d+1)}$. Therefore, an estimate of a convergence domain of (3.35) is given by

$$
\mathbb{D}=\left\{(x, y) \in \mathbb{C}^{2}: s(d+1)(|x|+|y|)+s^{2}(d+1)|x| y \mid<1\right\} .
$$

See Figure 3.1.

Inequality (3.30) shows that the series $\sum_{\substack{p=1 \\ m+n=p}}^{\infty} E_{m, n} x^{m} y^{n}$ will be absolutely convergent whenever $(x, y) \in \mathbb{D}$, which implies that the infinite product $L T R \prod_{\substack{q=1 \\ m+n=q}}^{\infty}\left(I+G_{m, n} x^{m} y^{n}\right)$ is at least absolutely convergent in the region $\mathbb{D}$. 
Relation (3.36) makes it possible to obtain a domain of absolute convergence in terms of polydiscs. Let $|x|<\rho$ and $|y|<\rho$. Inequality (3.36) implies that the MPPE2 will be absolutely convergent if

$$
s(d+1)(2 \rho)+s^{2}(d+1) \rho^{2}=(d+1)\left[(s \rho+1)^{2}-1\right]<1,
$$

or equivalently, if $\rho<s^{-1}\left[\sqrt{\frac{d+2}{d+1}}-1\right]$. The inequalities for $\rho$ obtained from the defining quadratic equation of $\mathbb{D}$, namely

$$
|x|<s^{-1}\left[\sqrt{\frac{d+2}{d+1}}-1\right], \quad|y|<s^{-1}\left[\sqrt{\frac{d+2}{d+1}}-1\right]
$$

are sharp in the sense that if

$$
x=-s^{-1}\left[\sqrt{\frac{d+2}{d+1}}-1\right], \quad y=-s^{-1}\left[\sqrt{\frac{d+2}{d+1}}-1\right],
$$

then $\left|s(d+1)(x+y)-s^{2}(d+1) x y\right|=1$ and the sum of the absolute values of the terms in the logarithmic power series of (3.35) diverge.

In summary, we have shown that

$$
\sum_{\substack{q=1 \\ m+n=q}}^{\infty} \log \left(I-E_{m, n} x^{m} y^{n}\right)=\log \left(I-\sum_{\substack{p=1 \\ m+n=p}}^{\infty} s^{m+n} W x^{m} y^{n}\right)
$$

will be absolutely convergent whenever $(x, y) \in \mathbb{D}$ or whenever $(x, y) \in D_{x \rho} \times D_{y \rho}$ with $\rho<s^{-1}\left[\sqrt{\frac{d+2}{d+1}}-1\right]$, where

$$
D_{x \rho}: \equiv\{x:|x|<\rho\}, \quad D_{y \rho}: \equiv\{y:|y|<\rho\} .
$$

Hence, $L T R \prod_{\substack{q=1 \\ m+n=q}}^{\infty}\left(I-E_{m, n} x^{m} y^{n}\right)$ will also be absolutely convergent for the same regions.

We can summarize what we have shown so far regarding the absolute convergence of MPPE2 in both Equations (3.25), (3.28) in term of spectral conditions and obtain the second major result of this section.

Theorem 3.5.2. (1.) Let $F(x, y)=I+\sum_{\substack{p=1 \\ m+n=p}}^{\infty} A_{m, n} x^{m} y^{n}$. Let $W$ and $s$ be as defined in Part (2.) of Theorem 3.5.1. Both F $(x, y)$ and its MPPE2,

$$
F(x, y)=I+\sum_{\substack{p=1 \\ m+n=p}}^{\infty} A_{m, n} x^{m} y^{n}=\operatorname{LTR} \prod_{\substack{q=1 \\ m+n=q}}^{\infty}\left(I+G_{m, n} x^{m} y^{n}\right),
$$


and the auxiliary function, along with its MPPE2,

$$
M(x, y)=I-\sum_{\substack{p=1 \\ m+n=p}}^{\infty} s^{m+n} W x^{m} y^{n}=L T R \prod_{\substack{q=1 \\ m+n=q}}^{\infty}\left(I-E_{m, n} x^{m} y^{n}\right)
$$

will be absolutely convergent whenever $(x, y) \in \mathbb{D}$, where

$$
\mathbb{D}=\left\{(x, y) \in \mathbb{C}^{2}:(d+1) s(|x|+|y|)+(d+1) s^{2}|x||y|<1\right\} .
$$

(2.) With the same conventions as in Part (1), both $F(x, y)$ and its MPPE2, along with $M(x, y)$ and its MPPE2, will be absolutely convergent whenever $(x, y) \in D_{x \rho} \times D_{y \rho}$ with $\rho<s^{-1}\left[\sqrt{\frac{d+2}{d+1}}-1\right]$, where

$$
D_{x \rho}: \equiv\{x:|x|<\rho\}, \quad D_{y \rho}: \equiv\{y:|y|<\rho\} .
$$

See Figure 3.1.

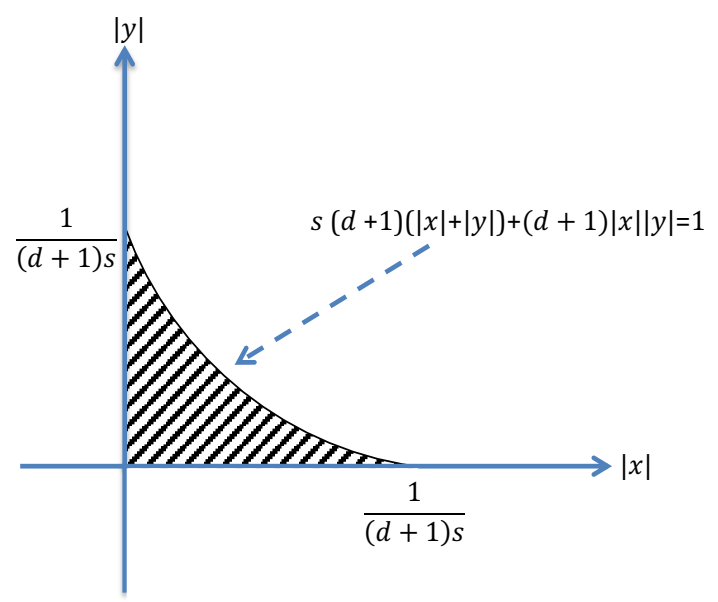

Figure 3.1: A domain of absolute convergence of Equations (3.25) and (3.28).

We should mention that for $F(x, y)=I+\sum_{\substack{p=1 \\ m+n=p}}^{\infty} A_{m, n} x^{m} y^{n}$, the results of Theorem 3.5.1(2) and Theorem 3.5.2 and can be stated and analogously proven with $s$ replaced by

$$
S:=\sup _{\substack{p=1 \\ m+n=p}}\left\|A_{m, n}\right\|^{\frac{1}{m+n}},
$$


Furthermore, the definition of $S$ in Equation (3.39) provides a connection between the domain of convergence for the majorizing MPPE2 of Theorem 3.5.1(2) and a scalar counterpart for the MPPE2 of $F(x, y)$ as seen in the following theorem:

Theorem 3.5.3. Given the matrix function $F(x, y)$

$$
F(x, y)=I+\sum_{\substack{p=1 \\ m+n=p}}^{\infty} A_{m, n} x^{m} y^{n}=\operatorname{LTR} \prod_{\substack{q=1 \\ m+n=q}}^{\infty}\left(I+G_{m, n} x^{m} y^{n}\right),
$$

with $A_{m, n}, G_{m, n} \in M_{d}(\mathbb{C})$, define the following auxiliary matrices functions with coeffcients over $M_{d}(\mathbb{R})$ :

$$
\begin{aligned}
& C(x, y)=I-\sum_{\substack{p=1 \\
m+n=p}}^{\infty}\left|A_{m, n}\right| x^{m} y^{n}=L T R \prod_{\substack{q=1 \\
m+n=q}}^{\infty}\left(I-C_{m, n} x^{m} y^{n}\right) \\
& M(x, y)=I-\sum_{\substack{p=1 \\
m+n=p}}^{\infty} M_{m, n} x^{m} y^{n}=L T R \prod_{\substack{q=1 \\
m+n=q}}^{\infty}\left(I-E_{m, n} x^{m} y^{n}\right) .
\end{aligned}
$$

Let $\|\cdot\|$ be a suitable matrix norm and assume that $\left|A_{m, n}\right| \leq M_{m, n}$ for all $(m, n) \in \mathbb{N}_{0} \times$ $\mathbb{N}_{0} \backslash\{(0,0)\}$. If $\sum_{\substack{p=1 \\ m+n=p}}^{\infty}|| E_{m, n}||\left|x^{m} y^{n}\right|$ converges absolutely in a domain $\mathbb{D}$, then the scalar function $\prod_{\substack{q=1 \\ m+n=q}}^{\infty}\left(1+\left\|G_{m, n}\right\| x^{m} y^{n}\right)$ also converges absolutely within $\mathbb{D}$.

Proof. Since $\left|A_{m, n}\right| \leq M_{m, n}$ for all $(m, n) \in \mathbb{N}_{0} \times \mathbb{N}_{0} \backslash\{(0,0)\}$, Theorem 3.5.1(1) implies that

$$
O \leq\left|G_{m, n}\right| \leq E_{m, n}
$$

Since the norm is suitable, the monotonicity of the matrix inequalities of (3.40) is preserved and implies following string of scalar inequalities

$$
0 \leq\left\|G_{m, n}\right\| \leq\left\|E_{m, n}\right\|
$$

from which the result follows.

\subsection{Matrix MPPE2's Induced by Scalar Functions}

In the next theorem we study the expansion of the matrix function $F(A x, A y)$, where $F(x, y)$ is a scalar analytic function. 
Theorem 3.6.1. Let $\left(a_{m, n}\right) \in \mathbb{C}$, where $(m, n) \in \mathbb{N}_{0} \times \mathbb{N}_{0} \backslash\{(0,0)\}$. Let

$$
F(x, y)=1+\sum_{\substack{p=1 \\ m+n=p}}^{\infty} a_{m, n} x^{m} y^{n}=\prod_{\substack{q=1 \\ m+n=q}}^{\infty}\left(1+g_{m, n} x^{m} y^{n}\right),
$$

where $g_{m, n} \in \mathbb{C},(m, n) \in \mathbb{N}_{0} \times \mathbb{N}_{0} \backslash\{(0,0)\}$ are scalar coefficients.

Let $A \in M_{d}(\mathbb{C})$. Consider the power series $F(A x, A y)$ together with its MPPE2 expansion

$$
F(A x, A y)=I+\sum_{\substack{p=1 \\ m+n=p}}^{\infty} a_{m, n} A^{m+n} x^{m} y^{n}=L T R \prod_{\substack{q=1 \\ m+n=q}}^{\infty}\left(I+g_{m, n} A^{m+n} x^{m} y^{n}\right) .
$$

Let $\left(\lambda_{i}\right)_{i=1}^{p}$ be the distinct eigenvalues of $A$. For $1 \leq i \leq p$, let $n_{i}$ be the algebraic multiplicity of $\lambda_{i}$, let $n_{i}^{g}$ be the geometric multiplicity of $\lambda_{i}$, and let $m_{i}$ be multiplicity of $\lambda_{i}$ as a linear factor within the minimal polynomial $m(\lambda)$. In other words,

$$
\operatorname{det}(\lambda I-A)=\prod_{i=1}^{p}\left(\lambda-\lambda_{i}\right)^{n_{i}} \quad m(\lambda)=\prod_{i=1}^{p}\left(\lambda-\lambda_{i}\right)^{m_{i}}
$$

Define

$$
\rho(A):=\max _{i}\left|\lambda_{i}\right| \quad i=1,2, \ldots, p, \quad s:=\sup _{m+n \geq 1}\left|a_{m, n}\right|^{\frac{1}{m+n}} .
$$

The MPPE2 of Equation (3.42) converges absolutely in the region

$$
\mathbb{D}=\left\{(x, y) \in \mathbb{C}^{2}: 2 s \rho(A)\|K\|(|x|+|y|)+2[s \rho(A)\|K\|]^{2}|x||y|<1\right\},
$$

with $K \in M_{d}(\mathbb{C})$,

$$
K=K_{1} \bigoplus K_{2} \bigoplus \cdots \bigoplus K_{p}
$$

where for $1 \leq i \leq p$,

$$
K_{i}=K_{i_{1}} \bigoplus K_{i_{2}} \bigoplus \cdots \bigoplus K_{i_{i}^{g}}
$$

with $K_{i_{j}}$ an $m_{i_{j}} \times m_{i_{j}}$ matrix as described below, $m_{i_{j}} \leq m_{i_{1}}=m_{i}$, and $\sum_{j=1}^{n_{i}^{g}} m_{i_{j}}=n_{i}$. Note if $m_{i_{j}}=1, K_{i_{j}}=[1]$, otherwise $K_{i_{j}}=I_{m_{i_{j}}}+N_{m_{i_{j}}}$ where $I_{m_{i_{j}}}$ is the $m_{i_{j}} \times m_{i_{j}}$ identity matrix, while $N_{m_{i j}}$ is the $m_{i_{j}} \times m_{i_{j}}$ the lower triangular nilpotent matrix associated with the Jordan block decomposition, i.e.

$$
K_{m_{i_{j}}}=I_{m_{i_{j}}}+N_{m_{i_{j}}}=\left(\begin{array}{cccccc}
1 & 0 & \ldots & \ldots & 0 & 0 \\
1 & 1 & 0 & \ldots & 0 & 0 \\
0 & 1 & 1 & \ddots & 0 & \vdots \\
\vdots & 0 & \ddots & \ddots & 0 & 0 \\
\vdots & \vdots & \ddots & \ddots & \ddots & 0 \\
0 & \ldots & 0 & 0 & 1 & 1
\end{array}\right) .
$$


Proof. It is known that given a matrix $A \in M_{d}(\mathbb{C})$ and an arbitrary small fixed $\varepsilon \leq \rho(A)$, there exists an invertible matrix $T$, such that

$$
J=T^{-1} A T,
$$

where

such that for $1 \leq i \leq p$,

$$
J=J_{1} \bigoplus J_{2} \bigoplus \cdots \bigoplus J_{p}
$$

$$
J_{i}=J_{i_{1}} \bigoplus J_{i_{2}} \bigoplus \cdots \bigoplus J_{i_{i_{i}^{g}}}
$$

with $J_{i_{j}}$ the $m_{i_{j}} \times m_{i_{j}}$ matrix as described below, $m_{i_{j}} \leq m_{i_{1}}=m_{i}$, and $\sum_{j=1}^{n_{i}^{g}} m_{i_{j}}=n_{i}$. If $m_{i_{j}}=1, J_{i_{j}}=\left[\lambda_{i}\right]$, otherwise $J_{i_{j}}=\lambda_{i} I_{m_{i_{j}}}+\varepsilon N_{m_{i_{j}}}$ where $I_{m_{i_{j}}}$ is the $m_{i_{j}} \times m_{i_{j}}$ identity matrix, while $N_{m_{i_{j}}}$ is the $m_{i_{j}} \times m_{i_{j}}$ the lower triangular nilpotent matrix associated with the Jordan block decomposition, i.e

$$
J_{i}=\left(\begin{array}{cccccc}
\lambda_{i} & 0 & \ldots & \ldots & 0 & 0 \\
\varepsilon & \lambda_{i} & 0 & \ldots & 0 & 0 \\
0 & \ddots & \ddots & \ddots & 0 & \vdots \\
\vdots & 0 & \ddots & \ddots & 0 & 0 \\
\vdots & \vdots & \ddots & \ddots & \ddots & 0 \\
0 & \ldots & 0 & 0 & \varepsilon & \lambda_{i}
\end{array}\right)=\lambda_{i} I_{m_{i_{j}}}+\varepsilon N_{m_{i_{j}}} .
$$

By using the similarity transformation of Equation (3.48), we have

$$
\begin{aligned}
& F(J x, J y)=I+\sum_{\substack{p=1 \\
m+n=p}}^{\infty} a_{m, n} J^{m+n} x^{m} y^{n}=I+\sum_{\substack{p=1 \\
m+n=p}}^{\infty} a_{m, n}\left(T^{-1} A T\right)^{m+n} x^{m} y^{n} \\
& =T^{-1}\left[I+\sum_{\substack{p=1 \\
m+n=p}}^{\infty} a_{m, n} A^{m+n} x^{m} y^{n}\right] T=L T R \prod_{\substack{q=1 \\
m+n=q}}^{\infty}\left(I+g_{m, n} J^{m+n} x^{m} y^{n}\right) \\
& =L T R \prod_{\substack{q=1 \\
m+n=q}}^{\infty}\left(I+g_{m, n}\left(T^{-1} A T\right)^{m+n} x^{m} y^{n}\right)=L T R \prod_{\substack{q=1 \\
m+n=q}}^{\infty} T^{-1}\left(I+g_{m, n} A^{m+n} x^{m} y^{n}\right) T \\
& =T^{-1}\left[L T R \prod_{\substack{q=1 \\
m+n=q}}^{\infty}\left(I+g_{m, n} A^{m+n} x^{m} y^{n}\right)\right] T=T^{-1}[F(A x, A y)] T .
\end{aligned}
$$

Consequently, the power series and the MPPE2 in Equation (3.42) converge absolutely if and only if the power series and its associated MPPE2 in

$$
F(J x, J y)=I+\sum_{\substack{p=1 \\ m+n=p}}^{\infty} a_{m, n} J^{m+n} x^{m} y^{n}=L T R \prod_{\substack{q=1 \\ m+n=q}}^{\infty}\left(I+g_{m, n} J^{m+n} x^{m} y^{n}\right),
$$


converge respectively. Consider two cases: in the first case assume that $\rho(A)=0$. As a result, $A$ is a nilpotent matrix and for some integer $P$ the equation $A^{(P+1)}=O$ holds. For $F(x, y)=1+\sum_{\substack{p=1 \\ m+n=p}}^{\infty} a_{m, n} x^{m} y^{n}, A$ nilpotent implies that

$$
F(A x, A y)=I+\sum_{\substack{p=1 \\ m+n=p}}^{P} a_{m, n} A^{m+n} x^{m} y^{n}=L T R \prod_{\substack{q=1 \\ m+n=q}}^{P}\left(I+g_{m, n} A^{m+n} x^{m} y^{n}\right),
$$

is a finite polynomial in $\mathbb{C}^{2}$.

Now consider the case that $\rho(A)>0$. Assuming $\varepsilon<\rho(A)$,

$$
|J| \leq \rho(A) K,
$$

where $K$ is defined in Equations (3.45) and (3.46). For $s=\sup _{m+n \geq 1}\left|a_{m, n}\right|^{\frac{1}{m+n}}$ and consider the scalar power series $M(x, y)$ together with its PPE2 expansion

$$
M(x, y)=1-\sum_{\substack{p=1 \\ m+n=p}}^{\infty} s^{m+n} x^{m} y^{n}=\prod_{\substack{q=1 \\ m+n=q}}^{\infty}\left(1-e_{m, n} x^{m} y^{n}\right),
$$

where $\left(e_{m, n}\right)$ is a sequence of non-negative real numbers determined by certain polynomials in $s$; see Theorem 1.4.1. By using (3.51), and assuming that $\|s \rho(A) K x\|<1$ and that $\|s \rho(A) K y\|<1$, we find that

$$
\begin{aligned}
& M(\rho(A) K x, \rho(A) K y)=I-\sum_{\substack{p=1 \\
m+n=p}}^{\infty}[s \rho(A) K]^{m+n} x^{m} y^{n} \\
& =I-\left[\sum_{m=0}^{\infty}[s \rho(A) K x]^{m} \sum_{n=0}^{\infty}[s \rho(A) K y]^{n}-I\right] \\
& =I-\left[(I-s \rho(A) K x)^{-1}(I-s \rho(A) K y)^{-1}-I\right] \\
& =I-(I-s \rho(A) K x)^{-1}(I-s \rho(A) K y)^{-1}[I-(I-s \rho(A) K x)(I-s \rho(A) K y)] \\
& =I-(I-s \rho(A) K x)^{-1}(I-s \rho(A) K y)^{-1}\left[s \rho(A) K(x+y)-[s \rho(A) K]^{2} x y\right] \\
& =(I-s \rho(A) K x)^{-1}(I-s \rho(A) K y)^{-1}\left[I-\left(2 s \rho(A) K(x+y)-2[s \rho(A) K]^{2} x y\right)\right] \\
& =L T R \prod_{q=1}^{\infty}\left(I-e_{m, n}[\rho(A) K]^{m+n} x^{m} y^{n}\right) . \\
& \quad \quad m+n=q
\end{aligned}
$$


Since $M(\rho(A) K x, \rho(A) K y)$ is a matrix function with commutative coefficients, the factors in the second to last line of (3.52) commute, and we may use the fact that $\log (A B)=$ $\log A+\log B$ and $\log A^{r}=r \log A$ to write

$$
\begin{aligned}
& \log (M(\rho(A) K x, \rho(A) K y))=\log \left[I-\sum_{\substack{p=1 \\
m+n=p}}^{\infty}[s \rho(A) K]^{m+n} x^{m} y^{n}\right] \\
& =\log \left[(I-s \rho(A) K x)^{-1}(I-s \rho(A) K y)^{-1}\left(I-\left(2 s \rho(A) K(x+y)-2[s \rho(A) K]^{2} x y\right)\right)\right] \\
& =-\log (I-s \rho(A) K x)-\log (I-s \rho(A) K y) \\
& \quad+\log \left(I-\left(2 s \rho(A) K(x+y)-2[s \rho(A) K]^{2} x y\right)\right) \\
& =\sum_{\ell=1}^{\infty} \frac{[s \rho(A) K x]^{\ell}}{\ell}+\sum_{\ell=1}^{\infty} \frac{[s \rho(A) K y]^{\ell}}{\ell}-\sum_{\ell=1}^{\infty} \frac{\left[2 s \rho(A) K(x+y)-2[s \rho(A) K]^{2} x y\right]^{\ell}}{\ell} .
\end{aligned}
$$

The three series in (3.53) are absolutely convergent for $|x||y|<\frac{1}{s \rho(A)\|K\|}$, and for $\| 2 s \rho(A) K(x+$ $y)-2[s \rho(A) K]^{2} x y \|<1$, respectively. By triangle inequality and the fact $\left\|K^{2}\right\| \leq\|K\|^{2}$, we have

$$
\begin{aligned}
\| 2 s \rho(A) K(x+y)-2[ & s \rho(A) K]^{2} x y \| \leq \\
& 2 s \rho(A)\|K\|(|x|+|y|)+2 s^{2} \rho^{2}(A)\|K\|^{2}|x||y| .
\end{aligned}
$$

If we require

$$
2 s \rho(A)\|K\|(|x|+|y|)+2[s \rho(A)\|K\|]^{2}|x||y|<1 .
$$

Since

$$
2 s \rho(A)\|K\||x|<2 s \rho(A)\|K\|(|x|+|y|)+2[s \rho(A)\|K\|]^{2}|x||y|<1,
$$

we have $|x|<\frac{1}{2 s \rho(A)\|K\|}<\frac{1}{s \rho(A)\|K\|}$, which is a condition for the validity of the calculations in (3.52). Similarly, $|y|<\frac{1}{2 s \rho(A)\|K\|}<\frac{1}{s \rho(A)\|K\|}$. Therefore, an estimate of a convergence domain of (3.53) is given by

$$
\mathbb{D}=\left\{(x, y) \in \mathbb{C}^{2}: s \rho(A)\|K\|(|x|+|y|)+2[s \rho(A)\|K\|]^{2}|x||y|<1\right\} .
$$

To determine the domain of convergence for the MPPE2's of Equation (3.50) we apply Theorem 3.5.1. In particular, take Equation (3.25) and set $A_{m, n}=a_{m, n} J^{m+n}$ and $G_{m, n}=g_{m, n} J^{m+n}$; then take Equation (3.27) and set $M_{m, n}=[s \rho(A) K]^{m+n}$ and $E_{m, n}=$ 
$e_{m, n}[\rho(A) K]^{m+n}$. Next recall that $s=\sup _{m+n \geq 1}\left|a_{m, n}\right|^{\frac{1}{m+n}}$. This definition, when combined with Inequality (3.51), shows that

$$
O \leq\left|A_{m, n}\right|=\left|a_{m, n}\right||J|^{m+n} \leq s^{m+n}|\rho(A) K|^{m+n}=M_{m, n} .
$$

Thus the conditions for Theorem 3.5.1 are satisfied and we deduce that

$$
O \leq\left|g_{m, n} J^{m+n}\right| \leq e_{m, n}[\rho(A) K]^{m+n} \leq-P_{m, n},
$$

where $P_{m, n}$ is the coefficient of $x^{m} y^{n}$ in the power series of $\log (M(\rho(A) K x, \rho(A) K y))$; see (3.53). Since our norm is suitable, we use (3.54) to deduce that

$$
0 \leq\left|g_{m, n}\right||| J^{m+n}\left\|\leq e_{m, n} \rho(A)^{m+n}\right\| K^{m+n}\|\leq\| P_{m, n} \| .
$$

The inequalities of (3.55), when combined with Proposition 3.5.1, imply that a domain of absolute convergence of $\log (M(\rho(A) K x, \rho(A) K y))$, namely the region $\mathbb{D}$, is also a domain of absolute convergence for $\operatorname{LTR} \prod_{\substack{q=1 \\ m+n=q}}^{\infty}\left(I+g_{m, n} J^{m+n} x^{m} y^{n}\right)$.

In the next theorem we study the expansion of the matrix function $F(B x, C y)$, where $F(x, y)$ is a scalar analytic function and $B, C \in M_{d}(\mathbb{C})$ are both diagonalizable and $B C=$ $C B \neq O$.

Theorem 3.6.2. Let $a_{m, n}$ be a sequence of complex numbers, where $(m, n) \in \mathbb{N}_{0} \times \mathbb{N}_{0} \backslash$ $\{(0,0)\}$. Let

$$
F(x, y)=1+\sum_{\substack{p=1 \\ m+n=p}}^{\infty} a_{m, n} x^{m} y^{n}=\prod_{\substack{q=1 \\ m+n=q}}^{\infty}\left(1+g_{m, n} x^{m} y^{n}\right),
$$

where $g_{m, n} \in \mathbb{C},(m, n) \in \mathbb{N}_{0} \times \mathbb{N}_{0} \backslash\{(0,0)\}$ are scalar coefficients.

Let $B$ and $C$ be two commuting $d$ by d diagonalizable matrices. Consider the power series $F(B x, C y)$ together with its MPPE2 expansion

$$
F(B x, C y)=I+\sum_{\substack{p=1 \\ m+n=p}}^{\infty} a_{m, n} B^{m} C^{n} x^{m} y^{n}=L T R \prod_{\substack{q=1 \\ m+n=q}}^{\infty}\left(I+g_{m, n} B^{m} C^{n} x^{m} y^{n}\right) .
$$

Let $\left(\lambda_{i}\right)_{i=1}^{p},\left(\lambda_{i}^{\prime}\right)_{i=1}^{p}$ be the distinct eigenvalues of $B, C$ respectively. Define

$$
\begin{aligned}
\rho(B) & :=\max _{i}\left|\lambda_{i}\right| \quad i=1,2, \ldots, p \\
\rho(C) & :=\max _{i}\left|\lambda_{i}^{\prime}\right| \quad i=1,2, \ldots, p \\
s & :=\sup _{m+n \geq 1}\left|a_{m, n}\right|^{\frac{1}{m+n}} .
\end{aligned}
$$


The MPPE2 in (3.57) converges absolutely in the region

$$
\mathbb{D}=\left\{(x, y) \in \mathbb{C}^{2}: 2 s[\rho(B)|x|+\rho(C)|y|]\|I\|+2 s^{2} \rho(B) \rho(C)\|I\||x||y|<1\right\},
$$

where I is a d by $d$ the identity matrix.

Proof. Let $B, C$ be two commute diagonalizable matrices. By using [[40], Proposition 6.2.6, Page 253] there is a single invertible matrix $S \in M_{d}(\mathbb{C})$ such that

$$
\begin{aligned}
& D_{1}=S^{-1} B S \\
& D_{2}=S^{-1} C S,
\end{aligned}
$$

are both diagonal, where

$$
D_{1}=\left(\begin{array}{cccccc}
\lambda_{1} & 0 & \ldots & \ldots & 0 & 0 \\
0 & \lambda_{2} & 0 & \ldots & 0 & 0 \\
0 & \ddots & \ddots & \ddots & 0 & \vdots \\
\vdots & 0 & \ddots & \ddots & 0 & 0 \\
\vdots & \vdots & \ddots & \ddots & \ddots & 0 \\
0 & \ldots & 0 & 0 & 0 & \lambda_{p}
\end{array}\right), D_{2}=\left(\begin{array}{cccccc}
\lambda_{1}^{\prime} & 0 & \ldots & \ldots & 0 & 0 \\
0 & \lambda_{2}^{\prime} & 0 & \ldots & 0 & 0 \\
0 & \ddots & \ddots & \ddots & 0 & \vdots \\
\vdots & 0 & \ddots & \ddots & 0 & 0 \\
\vdots & \vdots & \ddots & \ddots & \ddots & 0 \\
0 & \ldots & 0 & 0 & 0 & \lambda_{p}^{\prime}
\end{array}\right) .
$$

By using the similarity transformations (3.62) and (3.63), we have

$$
\begin{aligned}
& F\left(D_{1} x, D_{2} y\right)=I+\sum_{\substack{p=1 \\
m+n=p}}^{\infty} a_{m, n} D_{1}^{m} D_{2}^{n} x^{m} y^{n}=I+\sum_{\substack{p=1 \\
m+n=p}}^{\infty} a_{m, n}\left(S^{-1} B S\right)^{m}\left(S^{-1} C S\right)^{n} x^{m} y^{n} \\
& =S^{-1}\left[I+\sum_{\substack{p=1 \\
m+n=p}}^{\infty} a_{m, n} B^{m} C^{n} x^{m} y^{n}\right] S=L T R \prod_{\substack{q=1 \\
m+n=q}}^{\infty}\left(I+g_{m, n} D_{1}^{m} D_{2}^{n} x^{m} y^{n}\right) \\
& =\operatorname{LTR} \prod_{\substack{q=1 \\
m+n=q}}^{\infty}\left(I+g_{m, n}\left(S^{-1} B S\right)^{m}\left(S^{-1} C S\right)^{n} x^{m} y^{n}\right)=L T R \prod_{\substack{q=1 \\
m+n=q}}^{\infty} S^{-1}\left(I+g_{m, n} B^{m} C^{n} x^{m} y^{n}\right) S \\
& =S^{-1}\left[\operatorname{LTR} \prod_{\substack{q=1 \\
m+n=q}}^{\infty}\left(I+g_{m, n} B^{m} C^{n} x^{m} y^{n}\right)\right] S=S^{-1}[F(B x, C y)] S .
\end{aligned}
$$

Consequently, the power series and the MPPE2 in (3.57) converge absolutely if and only if the power series and its associated MPPE2 in

$$
F\left(D_{1} x, D_{2} y\right)=I+\sum_{\substack{p=1 \\ m+n=p}}^{\infty} a_{m, n} D_{1}^{m} D_{2}^{n} x^{m} y^{n}=L T R \prod_{\substack{q=1 \\ m+n=q}}^{\infty}\left(I+g_{m, n} D_{1}^{m} D_{2}^{n} x^{m} y^{n}\right)
$$


converge absolutely respectively. Consider two cases: in the first case, assume that $\rho(B)=$ 0 or $\rho(C)=0$. As a result, $B$ or $C$ is a nilpotent matrix and for some integer $P$ the equation $B^{(P+1)}=O$ or $C^{(P+1)}=O$ holds. For $F(x, y)=1+\sum_{\substack{p=1 \\ m+n=p}}^{\infty} A_{m, n} x^{m} y^{n}, B$ or $C$ nilpotent implies that

$$
F(B x, C y)=I+\sum_{\substack{p=1 \\ m+n=p}}^{P} a_{m, n} B^{m} C^{n} x^{m} y^{n}=L T R \prod_{\substack{q=1 \\ m+n=q}}^{P}\left(I+g_{m, n} B^{m} C^{n} x^{m} y^{n}\right),
$$

is a finite polynomial in $\mathbb{C}$.

Now consider the case that $\rho(B) \rho(C)>0$. Then we have

$$
\begin{aligned}
& \left|D_{1}\right| \leq \rho(B) I \\
& \left|D_{2}\right| \leq \rho(C) I,
\end{aligned}
$$

where $I$ is a $d$ by $d$ the identity matrix. For $s:=\sup _{m+n \geq 1}\left|a_{m, n}\right|^{\frac{1}{m+n}}$ and consider the scalar power series $M(x, y)$ together with its PPE2 expansion

$$
M(x, y)=1-\sum_{\substack{p=1 \\ m+n=p}}^{\infty} s^{m+n} x^{m} y^{n}=\prod_{\substack{q=1 \\ m+n=q}}^{\infty}\left(1-e_{m, n} x^{m} y^{n}\right) .
$$

where $\left(e_{m, n}\right)$ is a sequence of non-negative real numbers determined by certain polynomials in $s$; see Theorem 1.4.1. By using (3.65) and (3.66), and assuming that $\|s \rho(B) I x\|<1$ and $\|s \rho(C) I y\|<1$, we find that

$$
\begin{aligned}
& M(\rho(B) I x, \rho(C) I y)=I-\sum_{\substack{p=1 \\
m+n=p}}^{\infty} s^{m+n} \rho^{m}(B) \rho^{n}(C) I^{m} I^{n} x^{m} y^{n} \\
& =I-\left[\sum_{m=0}^{\infty}[s \rho(B) x I]^{m} \sum_{n=0}^{\infty}[s \rho(C) y I]^{n}-I\right] \\
& =I-\left[(I-s \rho(B) x I)^{-1}(I-s \rho(C) y I)^{-1}-I\right] \\
& =I-(I-s \rho(B) x I)^{-1}(I-s \rho(C) y I)^{-1}[I-(I-s \rho(B) x I)(I-s \rho(C) y I)] \\
& =I-(I-s \rho(B) x I)^{-1}(I-s \rho(C) y I)^{-1}\left[s(\rho(B) x+\rho(C) y) I-s^{2} \rho(B) \rho(C) x y I\right]
\end{aligned}
$$




$$
\begin{aligned}
& =(I-s \rho(B) x I)^{-1}(I-s \rho(C) y I)^{-1}\left[I-\left(2 s[\rho(B) x+\rho(C) y] I-2 s^{2} \rho(B) \rho(C) x y I\right)\right] \\
& =\operatorname{LTR} \prod_{\substack{q=1 \\
m+n=q}}^{\infty}\left(I-e_{m, n}[\rho(B) I]^{m}[\rho(C) I]^{n} x^{m} y^{n}\right) .
\end{aligned}
$$

Since $M(\rho(B) I x, \rho(C) I y)$ is a matrix with commutative coefficients, the factors in second to last line of (3.67) commute, and we may use the fact that $\log (A B)=\log A+\log B$ and $\log A^{r}=r \log A$ to write

$$
\begin{aligned}
& \log [M(\rho(B) I x, \rho(C) I y)]=\log \left[I-\sum_{\substack{p=1 \\
m+n=p}}^{\infty} s^{m+n} \rho^{m}(B) \rho^{n}(C) I^{m} I^{n} x^{m} y^{n}\right] \\
&=\log \left[(I-s \rho(B) I x)^{-1}(I-s \rho(C) I y)^{-1}(I-\{2 s[\rho(B) x+\rho(C) y] I-\right. \\
&\left.\left.\left.2 s^{2} \rho(B) \rho(C) I x y\right\}\right)\right] \\
&= \sum_{\ell=1}^{\infty} \frac{[s \rho(B) I x]^{\ell}}{\ell}+\sum_{\ell=1}^{\infty} \frac{[s \rho(C) I y]^{\ell}}{\ell}-\sum_{\ell=1}^{\infty} \frac{\left[2 s(\rho(B) x+\rho(C) y) I-2 s^{2} \rho(B) \rho(C) I x y\right]^{\ell}}{\ell} .
\end{aligned}
$$

The three series in (3.68) are absolutely convergent for $|x|<\frac{1}{s \rho(B)|| I||},|y|<\frac{1}{s \rho(C)|| I||}$, and for $\left\|2 s[\rho(B) x+\rho(C) y] I-2 s^{2} \rho(B) \rho(C) x y I\right\|<1$, respectively. By triangle inequality, we have

$$
\begin{aligned}
\| 2 s[\rho(B) x+ & \rho(C) y] I-2 s^{2} \rho(B) \rho(C) x y I \| \leq \\
& 2 s[\rho(B)|x|+\rho(C)|y|]\|I\|+2 s^{2} \rho(B) \rho(C)\|I\||| x|| y \mid .
\end{aligned}
$$

If we require

$$
2 s[\rho(B)|x|+\rho(C)|y|]|| I\left\|+2 s^{2} \rho(B) \rho(C)\right\| I \||| x|| y \mid<1 .
$$

Since

$$
2 s \rho(B)|x|\|I\|<2 s[\rho(B)|x|+\rho(C)|y|]|| I\left\|+2 s^{2} \rho(B) \rho(C)\right\| I \||| x|| y \mid<1,
$$

we have $|x|<\frac{1}{2 s \rho(B)|| I \|}<\frac{1}{s \rho(B)\|I\|}$, which is a condition for the validity of the calculations in (3.67). Similarly, $|y|<\frac{1}{2 s \rho(C)\|I\|}<\frac{1}{s \rho(C)\|I\|}$. Therefore, an estimate of a convergence domain of (3.68) is given by

$$
\mathbb{D}=\left\{(x, y) \in \mathbb{C}^{2}: 2 s[\rho(B)|x|+\rho(C)|y|]\|I\|+2 s^{2} \rho(B) \rho(C)\|I\||x||y|<1\right\} .
$$


To determine the domain of convergence for the MPPE2's of Equation (3.64) we apply Theorem 3.5.1. In particular, take Equation (3.25) and set $A_{m, n}=a_{m, n} D_{1}^{m} D_{2}^{n}$ and $G_{m, n}=g_{m, n} D_{1}^{m} D_{2}^{n}$; then take Equation (3.27) and set $M_{m, n}=[s \rho(B) I]^{m}[s \rho(C) I]^{n}$ and $E_{m, n}=e_{m, n}[\rho(B) I]^{m}[\rho(C) I]^{n}$. Next recall that $s=\sup _{m+n \geq 1}\left|a_{m, n}\right|^{\frac{1}{m+n}}$. This definition, when combined with Inequalities (3.65), (3.66), shows that

$$
O \leq\left|A_{m, n}\right|=\left|a_{m, n}\right|\left|D_{1}\right|^{m}\left|D_{2}\right|^{n} \leq s^{m+n}|\rho(B) I|^{m}|\rho(C) I|^{n}=M_{m, n} .
$$

Thus the conditions for Theorem 3.5.1 are satisfied and we deduce that

$$
O \leq\left|g_{m, n} D_{1}^{m} D_{2}^{n}\right| \leq e_{m, n}[\rho(B) I]^{m}[\rho(C) I]^{n} \leq-P_{m, n},
$$

where $P_{m, n}$ is the coefficient of $x^{m} y^{n}$ in the power series of $\log (M(\rho(B) I x, \rho(C) I y))$; see (3.68). Since our norm is suitable, we use (3.71) to deduce that

$$
0 \leq\left|g_{m, n}\right||| D_{1}^{m} D_{2}^{n}|| \leq e_{m, n} \rho(B)^{m} \rho(C)^{n}\|I\| \leq\left\|P_{m, n}\right\| .
$$

The inequalities of (3.72), when combined with Proposition 3.5.1, imply that a domain of absolute convergence of $\log (M(\rho(B) I x, \rho(C) I y))$, namely the region $\mathbb{D}$, which is defined in (3.70), is also a domain of absolute convergence for $\operatorname{LTR} \prod_{\substack{q=1 \\ m+n=q}}^{\infty}\left(I+g_{m, n} D_{1}^{m} D_{2}^{n} x^{m} y^{n}\right)$.

\subsection{Illustrative Examples}

In this section various examples are given to illustrate the main theorems of the previous sections. Our first example is an extension of Theorem 3.5.1.

Example 3.7.1. Fix $\alpha, \beta \in \mathbb{N}_{0}$. Consider the following matrix function $F(x, y)$ with its majorant matrix series:

$$
\begin{aligned}
& F(x, y)=I+\sum_{\substack{p=1 \\
u+v=p}}^{\infty} A_{u \alpha+\beta, v \alpha+\beta} x^{u \alpha+\beta} y^{v \alpha+\beta}=L T R \prod_{\substack{q=1 \\
m+n=q}}^{\infty}\left(I+G_{m, n} x^{m} y^{n}\right), \\
& C(x, y)=I-\sum_{\substack{p=1 \\
u+v=p}}^{\infty}\left|A_{u \alpha+\beta, v \alpha+\beta}\right| x^{u \alpha+\beta} y^{v \alpha+\beta}=\operatorname{LTR} \prod_{\substack{q=1 \\
m+n=q}}^{\infty}\left(I-C_{m, n} x^{m} y^{n}\right) \\
& M(x, y)=I-\sum_{\substack{p=1 \\
u+v=p}}^{\infty} s^{\alpha(u+v)+2 \beta} W x^{u \alpha+\beta} y^{v \alpha+\beta}=L T R \prod_{\substack{q=1 \\
m+n=q}}^{\infty}\left(I-E_{m, n} x^{m} y^{n}\right),
\end{aligned}
$$

where $A_{u \alpha+\beta, v \alpha+\beta}, W \in M_{d}(\mathbb{C}), A_{u \alpha+\beta, v \alpha+\beta}=\left(a_{\psi, \omega}(u \alpha+\beta, v \alpha+\beta)\right)_{\psi, \omega=1, \ldots, d}$,

$$
W=\left(w_{u, v}\right), \quad \text { with } \quad w_{u, v}=1, \quad v, u=1, \ldots, d,
$$


and

$$
s:=\sup _{\substack{\alpha, \beta \in \mathbb{N}_{0} \\ u+v \geq 1}}[a(u \alpha+\beta, v \alpha+\beta)]^{\frac{1}{\alpha(u+v)+2 \beta}}
$$

with

$$
a(u \alpha+\beta, v \alpha+\beta):=\max _{\psi, \omega}\left|a_{\psi, \omega}(u \alpha+\beta, v \alpha+\beta)\right|
$$

Since

$$
W=Q\left(\begin{array}{cccc}
d & 0 & \cdots & 0 \\
0 & 0 & 0 & \vdots \\
\vdots & 0 & \ddots & 0 \\
0 & \cdots & 0 & 0
\end{array}\right) Q^{T}
$$

The left hand side of Equation (3.73) becomes

$$
\begin{aligned}
& M(x, y)=I-\sum_{\substack{p=1 \\
u+v=p}}^{\infty} s^{\alpha(u+v)+2 \beta} W x^{u \alpha+\beta} y^{v \alpha+\beta} \\
& =I-\sum_{\substack{p=1 \\
u+v=p}}^{\infty} Q\left(\begin{array}{cccc}
d & 0 & \cdots & 0 \\
0 & 0 & 0 & \vdots \\
\vdots & 0 & \ddots & 0 \\
0 & \cdots & 0 & 0
\end{array}\right) Q^{T} s^{\alpha(u+v)+2 \beta} x^{u \alpha+\beta} y^{v \alpha+\beta} \\
& =Q\left[I-\sum_{\substack{p=1 \\
u+v=p}}^{\infty}\left(\begin{array}{cccc}
d & 0 & \cdots & 0 \\
0 & 0 & 0 & \vdots \\
\vdots & 0 & \ddots & 0 \\
0 & \cdots & 0 & 0
\end{array}\right) s^{\alpha(u+v)+2 \beta} x^{u \alpha+\beta} y^{v \alpha+\beta}\right] Q^{T} \\
& =Q\left[I-\left(\begin{array}{ccccc}
d \sum_{\substack{p=1 \\
u+v=p}}^{\infty} s^{\alpha(u+v)+2 \beta} x^{u \alpha+\beta} y^{v \alpha+\beta} & 0 & \cdots & 0 \\
& 0 & 0 & 0 & \vdots \\
& \vdots & 0 & \ddots & 0 \\
& 0 & 0 & \cdots & 0
\end{array}\right)\right] Q^{T} \\
& =Q\left(\begin{array}{cccc}
\phi(x, y) & 0 & \cdots & 0 \\
0 & 1 & 0 & \vdots \\
\vdots & 0 & \ddots & 0 \\
0 & 0 & \cdots & 1
\end{array}\right) Q^{T}
\end{aligned}
$$


where

$$
\begin{aligned}
\phi(x, y) & =1-d \sum_{\substack{p=1 \\
u+v=p}}^{\infty} s^{\alpha(u+v)+2 \beta} x^{u \alpha+\beta} y^{v \alpha+\beta} \\
& =1-d\left[\sum_{u=0}^{\infty}(s x)^{u \alpha+\beta} \sum_{v=0}^{\infty}(s y)^{v \alpha+\beta}-\left(s^{2} x y\right)^{\beta}\right] \\
& =1-d\left[\frac{(s x)^{\beta}}{1-(s x)^{\alpha}} \cdot \frac{(s y)^{\beta}}{1-(s y)^{\alpha}}-\left(s^{2} x y\right)^{\beta}\right] \\
& =1-\frac{d\left(s^{2} x y\right)^{\beta}\left[(s x)^{\alpha}+(s y)^{\alpha}-\left(s^{2} x y\right)^{\alpha}\right]}{\left[1-(s x)^{\alpha}\right]\left[1-(s y)^{\alpha}\right]} \\
& =\frac{1-\left[1+d\left(s^{2} x y\right)^{\beta}\right]\left[s^{\alpha}\left(x^{\alpha}+y^{\alpha}\right)-\left(s^{2} x y\right)^{\alpha}\right]}{\left[1-(s x)^{\alpha}\right]\left[1-(s y)^{\alpha}\right]} .
\end{aligned}
$$

Next we check for which values $(x, y)$ the series $\log [M(x, y)]=\sum_{\substack{p=1 \\ m+n=p}}^{\infty} P_{m, n} x^{m} y^{n}$ as defined in Equation (3.29) will be absolutely convergent. By taking the logarithm of Equation (3.74), we have

$$
\begin{aligned}
& \log [M(x, y)]=\log \left[I-\sum_{\substack{p=1 \\
u+v=p}}^{\infty} s^{\alpha(u+v)+2 \beta} W x^{u \alpha+\beta} y^{v \alpha+\beta}\right] \\
& =\log \left[Q\left(\begin{array}{cccc}
\phi(x, y) & 0 & \cdots & 0 \\
0 & 1 & 0 & \vdots \\
\vdots & 0 & \ddots & 0 \\
0 & \cdots & 0 & 1
\end{array}\right) Q^{T}\right] \\
& =Q\left(\begin{array}{cccc}
\log [\phi(x, y)] & 0 & \cdots & 0 \\
0 & \log 1 & 0 & \vdots \\
\vdots & 0 & \ddots & 0 \\
0 & 0 & \cdots & \log 1
\end{array}\right) Q^{T} \text {. }
\end{aligned}
$$

Therefore, it suffices to consider where the function $\log [\phi(x, y)]$ will be absolutely convergent. Observe that 


$$
\begin{aligned}
& \log [\phi(x, y)]=\log \left[1-d \sum_{\substack{p=1 \\
u+v=p}}^{\infty} s^{\alpha(u+v)+2 \beta} x^{u \alpha+\beta} y^{v \alpha+\beta}\right] \\
= & \log \left[\frac{1-\left[1+d\left(s^{2} x y\right)^{\beta}\right]\left[s^{\alpha}\left(x^{\alpha}+y^{\alpha}\right)-\left(s^{2} x y\right)^{\alpha}\right]}{\left[1-(s x)^{\alpha}\right]\left[1-(s y)^{\alpha}\right]}\right] \\
= & \log \left[1-\left[1+d\left(s^{2} x y\right)^{\beta}\right]\left[s^{\alpha}\left(x^{\alpha}+y^{\alpha}\right)-\left(s^{2} x y\right)^{\alpha}\right]\right] \\
& -\log \left[1-(s x)^{\alpha}\right]-\log \left[1-(s y)^{\alpha}\right] \\
= & \sum_{\ell=1}^{\infty} \frac{\left[(s x)^{\alpha}\right]^{\ell}}{\ell}+\sum_{\ell=1}^{\infty} \frac{\left[(s y)^{\alpha}\right]^{\ell}}{\ell} \\
& -\sum_{\ell=1}^{\infty} \frac{\left[\left[1+d\left(s^{2} x y\right)^{\beta}\right]\left[s^{\alpha}\left(x^{\alpha}+y^{\alpha}\right)-\left(s^{2} x y\right)^{\alpha}\right]\right]^{\ell}}{\ell}
\end{aligned}
$$

The three series in Equation (3.77) are absolutely convergent for $|x|<\frac{1}{s},|y|<\frac{1}{s}$, and for $\left|\left[1+d\left(s^{2} x y\right)^{\beta}\right]\left[s^{\alpha}\left(x^{\alpha}+y^{\alpha}\right)-\left(s^{2} x y\right)^{\alpha}\right]\right|<1$, respectively. By triangle inequality we have

$$
\begin{aligned}
& \left|\left[1+d\left(s^{2} x y\right)^{\beta}\right]\left[s^{\alpha}\left(x^{\alpha}+y^{\alpha}\right)-\left(s^{2} x y\right)^{\alpha}\right]\right| \leq \\
& {\left[1+d\left(s^{2}|x||y|\right)^{\beta}\right]\left[s^{\alpha}\left(|x|^{\alpha}+|y|^{\alpha}\right)+\left(s^{2}|x||y|\right)^{\alpha}\right] .}
\end{aligned}
$$

If we require

$$
\left[1+d\left(s^{2}|x||y|\right)^{\beta}\right]\left[s^{\alpha}\left(|x|^{\alpha}+|y|^{\alpha}\right)+\left(s^{2}|x||y|\right)^{\alpha}\right]<1,
$$

$|x|<\rho$, and $|y|<\rho$, then we have

$$
\left[1+d(s \rho)^{2 \beta}\right]\left[2(s \rho)^{\alpha}+(s \rho)^{2 \alpha}\right]<1
$$

Setting $w=s \rho$ in Equation (3.79) implies that

$$
\left(1+d w^{2 \beta}\right)\left(\left[w^{\alpha}+1\right]^{2}-1\right)<1,
$$

or equivalently,

$$
w^{2 \alpha}+2 w^{\alpha}+d w^{2 \alpha+2 \beta}+2 d w^{\alpha+2 \beta}<1 .
$$


Consider the moduli of all solutions $\rho$ of Equation (3.81). The smallest $\rho$ that solves this inequality equals the domain of convergence of the power series of $\log [M(x, y)]$. The solution to (3.81) is obtained in the following cases:

Case 1: Let $\alpha=1$ and $\beta=0$. Inequality (3.80) becomes

$$
(d+1)\left[(w+1)^{2}-1\right]<1,
$$

which ultimately implies that

$$
-1-\sqrt{\frac{d+2}{d+1}}<w<-1+\sqrt{\frac{d+2}{d+1}} .
$$

Consequently, the matrix function $F(x, y)$ and its associated MPPE2 converge absolutely whenever $(x, y) \in D_{x \rho} \times D_{y \rho}$ with $\rho<s^{-1}\left[\sqrt{\frac{d+2}{d+1}}-1\right]$, where

$$
D_{x \rho}: \equiv\{x:|x|<\rho\}, \quad D_{y \rho}: \equiv\{y:|y|<\rho\} .
$$

Note that this special case is Theorem 3.5.1.

Case2: Let $\alpha=2 \beta$, where $\alpha \neq 0$ and consider the special situation of $d=2$. Inequality (3.81) becomes

$$
2 w^{3 \alpha}+5 w^{2 \alpha}+2 w^{\alpha}<1
$$

By setting $t=w^{\alpha}$ in Equation (3.82), we have

$$
2 t^{3}+5 t^{2}+2 t-1<0
$$

or equivalently,

$$
(t+1)\left(2 t^{2}+3 t-1\right)<0
$$

Then we have

$$
t<\frac{-\sqrt{17}-3}{4} \text { or } \quad-1<t<\frac{\sqrt{17}-3}{4}
$$

which ultimately implies that

$$
w<\left[\frac{-\sqrt{17}-3}{4}\right]^{\frac{1}{\alpha}} \quad \text { or }[-1]^{\frac{1}{\alpha}}<w<\left[\frac{\sqrt{17}-3}{4}\right]^{\frac{1}{\alpha}} .
$$


Consequently, the matrix function $F(x, y)$ and its associated MPPE2 converge absolutely whenever $(x, y) \in D_{x \rho} \times D_{y \rho}$ with $\rho<s^{-1}\left[\frac{\sqrt{17}-3}{4}\right]^{\frac{1}{\alpha}}$, where

$$
D_{x \rho}: \equiv\{x:|x|<\rho\}, \quad D_{y \rho}: \equiv\{y:|y|<\rho\} .
$$

We turn to concrete examples where $F(x, y)$ is a scalar function.

Example 3.7.2. Let $\alpha \geq 0$ and $\theta_{m, n}$ be a sequence of real numbers, where $(m, n) \in \mathbb{N}_{0} \times$ $\mathbb{N}_{0} \backslash\{(0,0)\}$. Let

$$
F(x, y)=1+\sum_{\substack{p=1 \\ m+n=p}}^{\infty} \exp \left(i \theta_{m, n}\right)(m+n)^{\alpha} x^{m} y^{n}=\prod_{\substack{q=1 \\ m+n=q}}^{\infty}\left(1+g_{m, n} x^{m} y^{n}\right),
$$

where $g_{m, n} \in \mathbb{C}$. For any $A \in M_{d}(\mathbb{C})$, consider the power series $F(A x, A y)$ together with its MPPE2 expansion

$$
F(A x, A y)=I+\sum_{\substack{p=1 \\ m+n=p}}^{\infty} \exp \left(i \theta_{m, n}\right)(m+n)^{\alpha} A^{m+n} x^{m} y^{n}=L T R \prod_{\substack{q=1 \\ m+n=q}}^{\infty}\left(I+g_{m, n} A^{m+n} x^{m} y^{n}\right) .
$$

To determine a domain of convergence for the MPPE2 of Equation (3.85), we apply Theorem 3.6.1. This means we must determine the supremum of the sequence $a_{m, n}=$ $(m+n)^{\frac{1}{m+n}}$, where $(m, n) \in \mathbb{N}_{0} \times \mathbb{N}_{0} \backslash\{(0,0)\}$; this is equivalent to determining the supremum of the sequence $a_{n}=n^{\frac{1}{n}}$, where $n \in \mathbb{N}$. Since $\log x$ is increasing when $x \in \mathbb{R}^{+}$, and since $a_{n}>0$ for all $n \in \mathbb{N}$, we have

$$
\log \sup _{n \in \mathbb{N}} a_{n}=\sup _{n \in \mathbb{N}} \log a_{n}=\sup _{n \in \mathbb{N}} \frac{\log n}{n}
$$

Consider the function $g(x)=\frac{\log x}{x}$ on the interval $[1, \infty)$. Observe that

$$
g^{\prime}(x)=\frac{1-\log x}{x^{2}}
$$

which is positive on $[1, e)$ and negative on $(e, \infty)$ This means $g(x)$ is increasing on $[1, e)$ and decreasing on $(e, \infty)$, in particular its maximum occurs at $x=e$. But $e \notin \mathbb{N}$ so the supremum will be attained at either $n=2$ or $n=3$. In fact

$$
\frac{\log 2}{2}<\frac{\log 3}{3} \Longleftrightarrow 3 \log 2<2 \log 3 \Longleftrightarrow \log 8<\log 9
$$


Thus

$$
\sup _{n \in \mathbb{N}} \frac{\log n}{n}=\frac{\log 3}{3}
$$

and $\sup _{n \in \mathbb{N}} a_{n}=3^{\frac{1}{3}}$

Theorem 3.6.1 then implies that

$$
\mathbb{D}=\left\{(x, y) \in \mathbb{C}^{2}: 2\left(3^{\frac{\alpha}{3}}\right) \rho(A)\|K\|(|x|+|y|)+2\left[\left(3^{\frac{\alpha}{3}}\right) \rho(A)\|K\|\right]^{2}|x \| y|<1\right\}
$$

is the estimate for the domain of convergence.

Now let $B, C \in M_{d}(\mathbb{C})$ be two commuting diagonalizable matrices and consider the power series $F(B x, C y)$ together with its associated MPPE2 expansion

$$
\begin{aligned}
F(B x, C y) & =I+\sum_{\substack{p=1 \\
m+n=p}}^{\infty} \exp \left(i \theta_{m, n}\right)(m+n)^{\alpha}[B x]^{m}[C y]^{n} \\
& =L T R \prod_{\substack{q=1 \\
m+n=q}}^{\infty}\left(I+g_{m, n}[B x]^{m}[C y]^{n}\right)
\end{aligned}
$$

To determine the domain of convergence for the MPPE2 of Equation (3.87), we apply Theorem 3.6.2 with $s=3^{\frac{\alpha}{3}}$ to obtain

$$
\begin{aligned}
\mathbb{D}=\left\{(x, y) \in \mathbb{C}^{2}: 2\left(3^{\frac{\alpha}{3}}\right)\right. & \|I\|[\rho(B)|x|+\rho(C)|y|] \\
& \left.+2\left(3^{\frac{2 \alpha}{3}}\right) \rho(B) \rho(C)\|I\||x \| y|<1\right\} .
\end{aligned}
$$

Example 3.7.3. Let $p \in \mathbb{N}$ and consider the scalar bivariate exponential function together with its PPE2 expansion

$$
F(x, y)=\exp (x y)=1+\sum_{p=1}^{\infty} \frac{1}{p !}(x y)^{p}=\prod_{q=1}^{\infty}\left(1+g_{q}(x y)^{q}\right),
$$

where $g_{q} \in \mathbb{C}$. By the Jordan-Chevalley decomposition [ [30], page 17 ], every matrix $A \in M_{d}(\mathbb{C})$ can be uniquely decomposed into the sum of a diagonalizable and a nilpotent matrix

$$
A=V+Q
$$

The matrices $V, Q \in M_{d}(\mathbb{C})$ satisfy the following properties: 
1) $V$ is diagonalizable and $Q$ nilpotent, i.e.

$$
T^{-1} V T=D=\left(\begin{array}{cccc}
u_{1} & 0 & \cdots & 0 \\
0 & u_{2} & 0 & \vdots \\
\vdots & 0 & \ddots & 0 \\
0 & 0 & \cdots & u_{d}
\end{array}\right), Q^{d}=O
$$

where $u_{j}, j=1,2, \ldots, d$ are the $d$ eigenvalues of A counting multiplicities.

2) $V$ and $Q$ commute, i.e. $V Q=V Q$,

3) $V$ and $Q$ are polynomials in $A$, i.e. $\exists p(x)$ s.t $V=p(A)$ and $Q=A-p(A)$.

We will use this Jordan-Chevalley decomposition to rewrite $\exp \left(A^{2}\right)$ as

$$
\begin{aligned}
& \exp \left(A^{2}\right)=\prod_{q=1}^{\infty}\left(I+g_{q} A^{2 q}\right) \\
& =\exp [(V+Q)(V+Q)]=\exp \left[V^{2}+2 Q V+Q^{2}\right]=\exp \left(V^{2}\right) \exp (2 Q V) \exp \left(Q^{2}\right) \\
& =\left[\prod_{q=1}^{\infty}\left(I+g_{q} V^{2 q}\right)\right]\left[\prod_{q=1}^{d}\left(I+g_{q}(2 Q V)^{q}\right)\right]\left[\prod_{q=1}^{d}\left(I+g_{q} Q^{2 q}\right)\right] .
\end{aligned}
$$

To determine the domain of convergence for $\exp \left(A^{2}\right)=\prod_{q=1}^{\infty}\left(I+g_{q} A^{2 q}\right)$, it suffices to determine a domain of convergence for $\prod_{q=1}^{\infty}\left(I+g_{q} V^{2 q}\right)$. Since $\sup \left(\frac{1}{p !}\right)^{\frac{1}{p}}=1$, Theorem 3.6.1 implies an estimate for the domain of convergence of $\prod_{q=1}^{\infty}\left(I+g_{q} V^{2 q}\right)$ is

$$
\mathbb{D}=\left\{(x, y) \in \mathbb{C}^{2}: 4 \rho(V)\|K\|+2[\rho(V)\|K\|]^{2}<1\right\} .
$$

We may use this information to obtain an upper bound on the spectrum of $V$. Since $2[\rho(V)\|K\|]^{2}<1$, we deduce that

$$
\rho(V)<\frac{1}{\sqrt{2}|| K||} .
$$

A similar calculation shows that

$$
\rho(A)<\frac{1}{\sqrt{2}|| K \|} .
$$

Now let $B, C \in M_{d}(\mathbb{C})$ be two commuting diagonalizable matrices. Consider the power series $F(B x, C y)$ together with its MPPE2 expansion

$$
F(B x, C y)=\exp (B C x y)=I+\sum_{p=1}^{\infty} \frac{(B C)^{p}}{p !}(x y)^{p}=L T R \prod_{q=1}^{\infty}\left(I+g_{q}(B C x y)^{q}\right) .
$$


To determine the domain of convergence for $\exp (B C)=\prod_{q=1}^{\infty}\left(I+g_{q}(B C)^{q}\right)$, we use Theorem 3.6.2, along with $\sup \left(\frac{1}{p !}\right)^{\frac{1}{p}}=1$, to obtain the following estimate.

$$
\mathbb{D}=\left\{(x, y) \in \mathbb{C}^{2}: 2[\rho(B)+\rho(C)]\|I\|+2\|I\| \rho(B) \rho(C)<1\right\} .
$$

We may use this information to obtain an upper bound on the spectrum of $B$ and $C$. Since $2\|I\| \rho(B) \rho(C)<1$, we deduce that

$$
\rho(B), \rho(C)<\frac{1}{2\|I\|}
$$

Example 3.7.4. Let $A, B, C, \in M_{d}(\mathbb{C})$, where $B$ and $C$ are diagonalizable with $B C=C B \neq$ $O$. The techniques of Example 3.7.3 may also be applied to other matrix function such as

$$
\begin{aligned}
& I+\log \left(I-A^{2}\right), \quad A^{-2} \log \left(I-A^{2}\right), \quad \cos A^{2}, \quad I+\sin A^{2}, \quad A^{2} \sin A^{2}, \\
& \cosh A^{2}, \quad I+\sinh A^{2}, \quad A^{-2} \tan A^{2}, \quad \arccos A^{2}, \quad I+\arcsin A^{2}, \\
& I+\log (I-B C), \quad B C \log (I-B C), \quad \cos B C, \quad I+\sin B C, \quad B C \sin B C, \\
& \cosh B C, \quad I+\sinh B C, \quad B C \tan B C, \quad \arccos B C, \quad I+\arcsin B C .
\end{aligned}
$$

We will demonstrate these techniques for $\cos A^{2}, \cos B C, A^{-2} \sin A^{2}$, and $[B C]^{-1} \sin B C$, leaving the rest to the reader. Let $g_{q}$ and $\hat{g}_{q}$ be the scalar coefficients in the PPE2 expansions of the even scalar functions

$$
\begin{aligned}
& \cos x y=1+\sum_{p=1}^{\infty} \frac{(-1)^{p} x^{2 p} y^{2 p}}{(2 p) !}=\prod_{q=1}^{\infty}\left(1+g_{q} x^{2 q} y^{2 q}\right), \\
& \sin x y=x y\left[1+\sum_{p=1}^{\infty} \frac{(-1)^{p} x^{2 p} y^{2 p}}{(2 p+1) !}\right]=x y \prod_{q=1}^{\infty}\left(1+\hat{g}_{q} x^{2 q} y^{2 q}\right) .
\end{aligned}
$$

First,

$$
\cos A^{2}=I+\sum_{p=1}^{\infty} \frac{(-1)^{p} A^{4 p}}{(2 p) !}=\prod_{q=1}^{\infty}\left(I+g_{q} A^{4 q}\right) .
$$

Since $s:=\sup _{p \geq 1}\left|\frac{(-1)^{p}}{(2 p) !}\right|^{\frac{1}{p}}=\frac{1}{2}$, Theorem 3.6.1 implies an estimate for domain of convergence of $\prod_{q=1}^{\infty}\left(I+g_{q} A^{4 q}\right)$ is

$$
\mathbb{D}=\left\{(x, y) \in \mathbb{C}^{2}: 2 \rho(A)\|K\|+\frac{1}{2}[\rho(A)\|K\|]^{2}<1\right\} .
$$


We may use this information to obtain an upper bound on the spectrum of A. Since $\frac{1}{2}[\rho(A)\|K\|]^{2}<1$, we deduce that

$$
\rho(A)<\frac{\sqrt{2}}{\|K\|}
$$

For

$$
\cos B C=I+\sum_{p=1}^{\infty} \frac{(-1)^{p} B^{2 p} C^{2 p}}{(2 p) !}=\prod_{q=1}^{\infty}\left(I+g_{q} B^{2 q} C^{2 q}\right) .
$$

Theorem 3.6.2 implies an estimate for the domain of convergence of $\prod_{q=1}^{\infty}\left(1+g_{q} x^{2 q} y^{2 q}\right)$ is

$$
\mathbb{D}=\left\{(x, y) \in \mathbb{C}^{2}:[\rho(B)+\rho(C)]\|I\|+\frac{1}{2}\|I\| \rho(B) \rho(C)<1\right\} .
$$

We may use this information to obtain an upper bound on the spectrum of $B$ and $C$. Since $\frac{1}{2}\|I\| \rho(B) \rho(C)<1$, we deduce that

$$
\rho(B), \rho(C)<\frac{2}{\|I\|} .
$$

Next

$$
\sin A^{2}=A^{2}\left[I+\sum_{p=1}^{\infty} \frac{(-1)^{p} A^{4 p}}{(2 p+1) !}\right]=A^{2} \prod_{q=1}^{\infty}\left(I+\hat{g}_{q} A^{4 q}\right) .
$$

Since $s:=\sup _{p \geq 1}\left|\frac{(-1)^{p}}{(2 p+1) !}\right|^{\frac{1}{p}}=\frac{1}{6}$, Theorem 3.6.1 implies an estimate for domain of convergence of $\prod_{q=1}^{\infty}\left(I+\hat{g}_{q} A^{4 q}\right)$ is

$$
\mathbb{D}=\left\{(x, y) \in \mathbb{C}^{2}: \frac{2}{3} \rho(A)\|K\|+\frac{1}{18}[\rho(A)\|K\|]^{2}<1\right\} .
$$

We may use this information to obtain an upper bound on the spectrum of A. Since $\frac{1}{18}[\rho(A)\|K\|]^{2}<1$, we deduce that

$$
\rho(A)<\frac{3 \sqrt{2}}{\|K\|} .
$$

Finally it can be shown that an estimate for the domain of convergence of

$$
\sin B C=B C\left[I+\sum_{p=1}^{\infty} \frac{(-1)^{p} B^{2 p} C^{2 p}}{(2 p+1) !}\right]=B C \prod_{q=1}^{\infty}\left(I+\hat{g}_{q} B^{2 p} C^{2 p}\right),
$$


is

$$
\mathbb{D}=\left\{(x, y) \in \mathbb{C}^{2}: \frac{1}{3}[\rho(B)+\rho(C)]\|I\|+\frac{1}{18}\|I\| \rho(B) \rho(C)<1\right\}
$$

and that

$$
\rho(B), \rho(C)<\frac{18}{\|I\|}
$$

Example 3.7.5. Consider the scalar bivariate exponential function together with its PPE2 expansion

$$
F(x, y)=\exp (x+y)=1+\sum_{\substack{p=1 \\ m+n=p}}^{\infty} \frac{1}{m ! n !} x^{m} y^{n}=\prod_{\substack{q=1 \\ m+n=q}}^{\infty}\left(1+g_{m, n} x^{m} y^{n}\right),
$$

where $g_{m, n} \in \mathbb{C}$. By the Jordan-Chevalley decomposition, every matrix $A \in M_{d}(\mathbb{C})$ can be uniquely decomposed into the sum of a diagonalizable and a nilpotent matrix

$$
A=V+Q
$$

The matrices $V, Q \in M_{d}(\mathbb{C})$ satisfy the following properties:

1) $V$ is diagonalizable and $Q$ nilpotent, i.e.

$$
T^{-1} V T=D=\left(\begin{array}{cccc}
u_{1} & 0 & \cdots & 0 \\
0 & u_{2} & 0 & \vdots \\
\vdots & 0 & \ddots & 0 \\
0 & 0 & \cdots & u_{d}
\end{array}\right), Q^{d}=O
$$

where $u_{j}, j=1,2, \ldots, d$ are the $d$ eigenvalues of A counting multiplicities.

2) $V$ and $Q$ commute, i.e. $V Q=V Q$,

3) $V$ and $Q$ are polynomials in $A$, i.e. $\exists p(x)$ s.t $V=p(A)$ and $Q=A-p(A)$.

We will use this Jordan-Chevalley decomposition to rewrite $\exp (2 A)$ as

$$
\begin{aligned}
\exp (2 A) & =\prod_{\substack{q=1 \\
m+n=q}}^{\infty}\left(I+g_{m, n} A^{m+n}\right) \\
& =\exp (2 V+2 Q)=\exp (2 V) \exp (2 Q) \\
& =\left[\prod_{\substack{q=1 \\
m+n=q}}^{\infty}\left(I+g_{m, n} V^{m+n}\right)\right]\left[\prod_{\substack{q=1 \\
m+n=q}}^{d}\left(I+g_{m, n} Q^{m+n}\right)\right] .
\end{aligned}
$$


To determine the domain of convergence for $\exp (2 A)=\prod_{\substack{q=1 \\ m+n=q}}^{\infty}\left(I+g_{m, n} A^{m+n}\right)$, it suffices to determine a domain of convergence for $\prod_{\substack{q=1 \\ m+n=q}}^{\infty}\left(I+g_{m, n} V^{m+n}\right)$. Since $\sup _{m+n \geq 1}\left[\frac{1}{m ! n !}\right]^{\frac{1}{m+n}}=1$, Theorem 3.6.1 implies an estimate for the domain of convergence of $\prod_{\substack{q=1 \\ m+n=q}}^{\infty}\left(I+g_{m, n} V^{m+n}\right)$ is

$$
\mathbb{D}=\left\{(x, y) \in \mathbb{C}^{2}: 4 \rho(V)\|K\|+2[\rho(V)\|K\|]^{2}<1\right\} .
$$

We may use this information to obtain an upper bound on the spectrum of $V$. Since $2[\rho(V)\|K\|]^{2}<1$, we deduce that

$$
\rho(V)<\frac{1}{\sqrt{2}|| K||}
$$

A similar calculation shows that

$$
\rho(A)<\frac{1}{\sqrt{2}|| K \|}
$$

Now let $B, C \in M_{d}(\mathbb{C})$ be two commuting diagonalizable matrices. Consider the power series $F(B x, C y)$ together with its MPPE2 expansion

$$
\begin{aligned}
F(B x, C y)=\exp (B x+C y) & =I+\sum_{\substack{p=1 \\
m+n=p}}^{\infty} \frac{B^{m} C^{n}}{m ! n !} x^{m} y^{n} \\
& =L T R \prod_{\substack{q=1 \\
m+n=q}}^{\infty}\left(I+g_{m, n} B^{m} C^{n} x^{m} y^{n}\right) .
\end{aligned}
$$

To determine the domain of convergence for $\exp (B+C)=\prod_{\substack{q=1 \\ m+n=q}}^{\infty}\left(I+g_{m, n} B^{m} C^{n}\right)$, we use Theorem 3.6.2, along with $\sup _{m+n \geq 1}\left[\frac{1}{m ! n !}\right]^{\frac{1}{m+n}}=1$, to obtain the following estimate:

$$
\mathbb{D}=\left\{(x, y) \in \mathbb{C}^{2}: 2[\rho(B)+\rho(C)]\|I\|+2\|I\| \rho(B) \rho(C)<1\right\} .
$$

We may use this information to obtain an upper bound on the spectrum of $B$ and $C$. Since $2\|I\| \rho(B) \rho(C)<1$, we deduce that

$$
\rho(B), \rho(C)<\frac{1}{2\|I\|} .
$$


Example 3.7.6. Let $A, B, C, \in M_{d}(\mathbb{C})$, where $B$ and $C$ are diagonalizable with $B C=C B \neq$ $O$. The techniques of Example 3.7.5 may also be applied to other matrix function such as

$$
\begin{aligned}
& I+\log (I-2 A), \quad(2 A)^{-1} \log (I-2 A), \quad \cos 2 A, \quad I+\sin 2 A, \quad 2 A \sin 2 A, \\
& \cosh 2 A, \quad I+\sinh 2 A, \quad(2 A)^{-1} \tan 2 A, \quad \arccos 2 A, \quad I+\arcsin 2 A, \\
& I+\log (I-[B+C]), \quad[B+C] \log (I-[B+C]), \quad \cos B+C, \quad I+\sin (B+C), \\
& {[B+C] \sin (B+C), \quad \cosh (B+C), \quad I+\sinh [B+C], \quad[B+C] \tan (B+C),} \\
& \arccos (B+C), \quad I+\arcsin (B+C) .
\end{aligned}
$$

We will demonstrate these techniques for $\cos 2 A, \cos (B+C),(2 A)^{-1} \sin 2 A$, and $[B+$ $C]^{-1} \sin (B+C)$, leaving the rest to the reader. Let $g_{m, n}$ and $\hat{g}_{m, n}$ be the scalar coefficients in the PPE2 expansions of the even scalar functions

$$
\begin{aligned}
\cos (x+y) & =1+\sum_{\substack{p=1 \\
m+n=2 p}}^{\infty} \frac{(-1)^{\frac{m+n}{2}}}{m ! n !} x^{m} y^{n}=\prod_{\substack{q=1 \\
m+n=2 q}}^{\infty}\left(1+g_{m, n} x^{m} y^{n}\right) \\
\sin (x+y) & =(x+y)\left[1+(x+y)^{-1} \sum_{n=1}^{\infty} \frac{(-1)^{n}}{(2 n+1) !}(x+y)^{2 n+1}\right] \\
& =(x+y)\left[1+\sum_{n=1}^{\infty} \frac{(-1)^{n}}{(2 n+1) !} \sum_{j=0}^{2 n}\left(\begin{array}{c}
2 n \\
j
\end{array}\right) x^{j} y^{2 n-j}\right] \\
& =(x+y)\left[1+\sum_{\substack{p=1 \\
m+n=2 p}}^{\infty} \frac{(-1)^{\frac{m+n}{2}}}{(m+n+1) !}\left(\begin{array}{c}
m+n \\
m
\end{array}\right) x^{m} y^{n}\right] \\
& =(x+y) \prod_{\substack{q=1 \\
m+n=2 q}}^{\infty}\left(1+\hat{g}_{m, n} x^{m} y^{n}\right) .
\end{aligned}
$$

First,

$$
\cos 2 A=I+\sum_{\substack{p=1 \\ m+n=2 p}}^{\infty} \frac{(-1)^{\frac{m+n}{2}}}{m ! n !} A^{m+n}=\prod_{\substack{q=1 \\ m+n=2 q}}^{\infty}\left(I+g_{m, n} A^{m+n}\right) .
$$

To determine a domain of convergence for the MPPE2 of Equation (3.97), we apply Theorem 3.6.1. This means we must determine the supremum of the sequence

$$
a_{m, n}=\left\{[1 / m ! n !]^{1 /(m+n)} \text {, where } m+n \text { is a positive even number }\right\} \text {. }
$$

Since

$$
\left(\frac{1}{m ! n !}\right)^{1 /(m+n)} \leq\left(\frac{1}{1 ! 1 !}\right)^{1 /(m+n)} \leq\left(\frac{1}{1}\right)^{1 / 2}=1,
$$


and this sequence attains the value of 1 when $p=1$ and $m=1=n$, we conclude that $s:=\sup _{\substack{p \geq 1 \\ m+n=2 p}}\left|\frac{(-1)^{\frac{m+n}{2}}}{m ! n !}\right|^{\frac{1}{m+n}}=1$. Therefore, Theorem 3.6.1 implies an estimate for domain of convergence of $\prod_{\substack{q=1 \\ m+n=2 q}}^{\infty}\left(I+g_{m, n} A^{m+n}\right)$ is

$$
\mathbb{D}=\left\{(x, y) \in \mathbb{C}^{2}: 4 \rho(A)\|K\|+2[\rho(A)\|K\|]^{2}<1\right\} .
$$

We may use this information to obtain an upper bound on the spectrum of A. Since $2[\rho(A)\|K\|]^{2}<1$, we deduce that

$$
\rho(A)<\frac{1}{\sqrt{2}|| K \|}
$$

For

$$
\cos (B+C)=I+\sum_{\substack{p=1 \\ m+n=2 p}}^{\infty} \frac{(-1)^{\frac{m+n}{2}}}{m ! n !} B^{m} C^{n}=\prod_{\substack{q=1 \\ m+n=2 q}}^{\infty}\left(I+g_{m, n} B^{m} C^{n}\right)
$$

Theorem 3.6.2 implies an estimate for the domain of convergence of $\prod_{\substack{q=1 \\ m+n=2 q}}^{\infty}\left(I+g_{m, n} B^{m} C^{n}\right)$ is

$$
\mathbb{D}=\left\{(x, y) \in \mathbb{C}^{2}: 2[\rho(B)+\rho(C)]\|I\|+2\|I\| \rho(B) \rho(C)<1\right\} .
$$

We may use this information to obtain an upper bound on the spectrum of $B$ and $C$. Since $2 \rho(B) \rho(C)\|I\|<1$, we deduce that

$$
\rho(B), \rho(C)<\frac{1}{2\|I\|}
$$

Next

$$
\sin 2 A=2 A\left[I+\sum_{\substack{p=1 \\
m+n=2 p}}^{\infty} \frac{(-1)^{\frac{m+n}{2}}}{(m+n+1) !}\left(\begin{array}{c}
m+n \\
m
\end{array}\right) A^{m+n}\right]=2 A \prod_{\substack{q=1 \\
m+n=2 q}}^{\infty}\left(I+\hat{g}_{m, n} A^{m+n}\right) .
$$

To determine a domain of convergence for the MPPE2 of Equation (3.99), we apply Theorem 3.6.1. This means we must determine the supremum of the sequence

$$
a_{m, n}=\left\{\left[\left(\begin{array}{c}
m+n \\
m
\end{array}\right) /(m+n+1) !\right]^{\frac{1}{(m+n)}} \text {, where } m+n \text { is a positive even number }\right\} .
$$


Since

$$
\begin{aligned}
\left(\frac{\left(\begin{array}{c}
m+n \\
m
\end{array}\right)}{(m+n+1) !}\right)^{\frac{1}{(m+n)}} & =\left(\frac{1}{(m+n+1) m ! n !}\right)^{\frac{1}{(m+n)}} \leq\left(\frac{1}{m+n+1}\right)^{\frac{1}{(m+n)}} \\
& \leq\left(\frac{1}{m+n+1}\right)^{\frac{1}{2}} \leq\left(\frac{1}{3}\right)^{\frac{1}{2}}
\end{aligned}
$$

and the sequence obtains the value of $\left(\frac{1}{3}\right)^{\frac{1}{2}}$ when $p=1$ and $m=n=1$, we deduce that $s:=$ $\sup _{\substack{p=1 \\ m+n=2 p}}\left|\frac{(-1)^{\frac{m+n}{2}}}{(m+n+1) !}\left(\begin{array}{c}m+n \\ m\end{array}\right)\right|^{\frac{1}{m+n}}=\frac{1}{\sqrt{3}}$. Hence Theorem 3.6.1 implies an estimate for domain of convergence of $\prod_{\substack{q=1 \\ m+n=2 q}}^{\infty}\left(I+\hat{g}_{m, n} A^{m+n}\right)$ is

$$
\mathbb{D}=\left\{(x, y) \in \mathbb{C}^{2}: \frac{4}{\sqrt{3}} \rho(A)\|K\|+\frac{2}{3}[\rho(A)\|K\|]^{2}<1\right\} .
$$

We may use this information to obtain an upper bound on the spectrum of A. Since $\frac{2}{3}[\rho(A)\|K\|]^{2}<1$, we deduce that

$$
\rho(A)<\frac{\sqrt{3}}{\sqrt{2}|| K \|} .
$$

Finally it can be shown that an estimate for the domain of convergence of

$$
\begin{aligned}
\sin (B+C) & =[B+C]\left[I+\sum_{\substack{p=1 \\
m+n=2 p}}^{\infty} \frac{(-1)^{\frac{m+n}{2}}}{(m+n+1) !}\left(\begin{array}{c}
m+n \\
m
\end{array}\right) B^{m} C^{n}\right] \\
& =[B+C] \prod_{\substack{q=1 \\
m+n=2 q}}^{\infty}\left(I+\hat{g}_{m, n} B^{m} C^{n}\right),
\end{aligned}
$$

is

$$
\mathbb{D}=\left\{(x, y) \in \mathbb{C}^{2}: \frac{2}{\sqrt{3}}[\rho(B)+\rho(C)]\|I\|+\frac{2}{3}\|I\| \rho(B) \rho(C)<1\right\}
$$

and that

$$
\rho(B), \rho(C)<\frac{3}{2\|I\|}
$$




\section{Chapter 4}

\section{Factorization of Bivariate Matrix Power Series via Power Inverse Product Expansion}

\subsection{Introduction}

Given $F(x, y)=I+\sum_{\substack{p=1 \\ m+n=p}}^{\infty} A_{m, n} x^{m} y^{n}$ with matrix coefficients, where either the defining expression for $F(x, y)$ is treated as a formal power series expansion or $F(x, y)$ is an analytic function with $F(0,0)=I$, the right side of

$$
F(x, y)=\prod_{\substack{q=1 \\ m+n=q}}^{\infty}\left(I-H_{m, n} x^{m} y^{n}\right)^{-1}
$$

is defined to be inverse matrix power product expansion in two independent variables, (denoted IMPPE2).

Next, we define what we mean by the inverse matrix power product expansion of $F(x, y)=$ $I+\sum_{\substack{p=1 \\ m+n=p}}^{\infty} A_{m, n} x^{m} y^{n}$. First we must interpret the inverse of an elementary factor as

$$
\left(I+G_{m, n} x^{m} y^{n}\right)^{-1}=I+\sum_{\alpha=1}^{\infty}(-1)^{\alpha} G_{m, n}^{\alpha} x^{\alpha m} y^{\alpha n},
$$

where the right hand side is a formal geometric series. 
Definition 4.1.1. Given $F(x, y)=I+\sum_{\substack{p=1 \\ m+n=p}}^{\infty} A_{m, n} x^{m} y^{n}$, a formal power series with matrix coefficients or an analytic function of two independent complex variables with $F(0,0)=I$, we say $F(x, y)$ has a left to right (canonically ordered) inverse matrix power product expansion in two independent variables if

$$
\begin{aligned}
& F(x, y)=\operatorname{LTR} \prod_{\substack{q=1 \\
m+n=q}}^{\infty}\left(I-H_{m, n} x^{m} y^{n}\right)^{-1} \\
& =\left(I-H_{1,0} x^{1} y^{0}\right)^{-1}\left(I-H_{0,1} x^{0} y^{1}\right)^{-1}\left(I-H_{2,0} x^{2} y^{0}\right)^{-1}\left(I-H_{1,1} x^{1} y^{1}\right)^{-1} \ldots,
\end{aligned}
$$

where the ordering of the right side follows the conventions of Definition 1.2.1. We say $F(x, y)$ has a right to left (reversed canonically ordered) inverse matrix power product expansion in two independent variables if

$$
\begin{aligned}
& F(x, y)=R T L \prod_{\substack{q=1 \\
m+n=q}}^{\infty}\left(I-H_{m, n} x^{m} y^{n}\right)^{-1} \\
= & \ldots\left(I-H_{k_{1}, k_{2}} x^{k_{1}} y^{k_{2}}\right)^{-1} \ldots\left(I-H_{2,0} x^{2} y^{0}\right)^{-1}\left(I-H_{0,1} x^{0} y^{1}\right)^{-1}\left(I-H_{1,0} x^{1} y^{0}\right)^{-1},
\end{aligned}
$$

where the ordering of the right side follows the conventions of Definition 1.2.1 when read from right to left. We refer to the right hand side of either Equation (4.2) or (4.3) as an IMPPE2.

The purpose of this chapter is to obtain both algebraic and analytic theorems for the IMPPE2 expansion of $F(x, y)=I+\sum_{\substack{p=1 \\ m+n=p}}^{\infty} A_{m, n} x^{m} y^{n}$. The three main results are as follows:

1. An algebraic structure property for $\left(H_{m, n}\right)$ in terms of recursive "mixed expansions"; see Theorem 4.3.2.

2. A domain of convergence criteria for the IMPPE2 in terms of a "majorizing" infinite product; see Theorem 4.4.1.

3. A domain of convergence criteria for the IMPPE2's by norm criteria; see Theorem 4.4.3.

The outline of this chapter is as follows. In Section 2 we study the expansion of a power series into a MPPE2 and provide an algebraic representation for the coefficients $G_{m, n}$ as a multivariate polynomials in $\left(A_{m, n}\right)_{\substack{m, n=0 \\ m+n=1}}^{\infty}$. In Section 3 we provide another way to recursively express the coefficients $H_{m, n}$ as a multivariate polynomial of the variables 
$A_{m, n}$. The algebraic result of Section 3 reveals an intriguing property of these expansions. If $A_{m, n} \leq 0$, then the coefficients $G_{m, n}$ in the IMPPE2 are non-positive. Section 4 exploits the non-positivity result of Section 4 to determine convergence conditions of the IMPPE2 in terms of a majorizing power product by focusing on spectral criteria. Moreover, at the end of Section 4, we employ norm criteria to analyze the convergence of IMPPE2. Section 5 is devoted to the study of the matrix IMPPE2 induced by scalar functions. Finally, in Section 7 six examples are presented to illustrate the applicability of the section 5 theorems.

\subsection{Algebraic Formulas for the Coefficients of In- verse Matrix Power Product Expansion}

In this section and the next we study the expansion of a two variable power series into an IMPPE2 and provide two algebraic representations for the coefficients $H_{m, n}$ as polynomials of the $\left(A_{m, n}\right)_{m, n}$. The first formula is almost an immediate consequence of Equation (4.2). Let

$$
F(x, y)=I+\sum_{\substack{p=1 \\ m+n=p}}^{\infty} A_{m, n} x^{m} y^{n}=\operatorname{LTR} \prod_{\substack{q=1 \\ m+n=q}}^{\infty}\left(I-H_{m, n} x^{m} y^{n}\right)^{-1} .
$$

By expanding the IMPPE2 of Equation (4.4) into a formal power series coefficient comparison shows that

$$
A_{m, n}=\sum_{\substack{i_{1}+i_{2}+\cdots+i_{r}=m \\ j_{1}+j_{2}+\cdots+j_{r}=n \\(1,0) \preceq\left(i_{1}, j_{1}\right) \preceq\left(i_{2}, j_{2}\right) \preceq \cdots \preceq\left(i_{r}, j_{r}\right) \preceq(m, n)}} H_{i_{1}, j_{1}} H_{i_{2}, j_{2}} \ldots H_{i_{r}, j_{r}},
$$

or equivalently that

$$
H_{m, n}=A_{m, n}-\left(\sum_{\substack{i_{1}+i_{2}+\cdots+i_{r}=m \\ j_{1}+j_{2}+\cdots+j_{r}=n \\(1,0) \preceq\left(i_{1}, j_{1}\right) \preceq\left(i_{2}, j_{2}\right) \preceq \cdots \preceq\left(i_{r}, j_{r}\right) \prec(m, n)}} H_{i_{1}, j_{1}} H_{i_{2}, j_{2}} \ldots H_{i_{r}, j_{r}}\right),
$$

where the summation runs over all partitions of $(m, n)$ into unrestricted parts.

\subsection{Structure Property of the Coefficients of an In- verse Power Product Expansion}

Just as we did in Section 2, we can recursively express the coefficients $H_{m, n}$ as a multivariate polynomial of the variables $A_{m, n}$. The ultimate result of this methodology is 
the structure property, Theorem 4.3.2, a crucial result for using proving convergence domains of power product expansions via majorizing IMPPE2. We begin with setting $A_{m, n}=B_{(1,0),(m, n)}$ and rewriting Equation (4.4) as

$$
\begin{aligned}
F(x, y) & =I+\sum_{\substack{p=1 \\
m+n=p}}^{\infty} B_{(1,0),(m, n)} x^{m} y^{n} \\
& =\left(I-H_{1,0} x\right)^{-1}\left[L T R \prod_{\substack{q=1 \\
m+n=q \\
(m, n) \succeq(0,1)}}^{\infty}\left(I-H_{m, n} x^{m} y^{n}\right)^{-1}\right] \\
& =\left(I-H_{1,0} x\right)^{-1}\left[I+\sum_{\substack{p=1 \\
m+n=p \\
(m, n) \succeq(0,1)}}^{\infty} B_{(0,1),(m, n)} x^{m} y^{n}\right],
\end{aligned}
$$

where the summation conventions follow Definition 1.2.1.

By continuing this procedure inductively we find that

$$
\begin{gathered}
I+\sum_{\substack{p=\ell \\
m+n=p \\
(m, n) \succeq\left(i_{k}, j_{k}\right)}}^{\infty} B_{\left(i_{k}, j_{k}\right),(m, n)} x^{m} y^{n}=\left(I-H_{i_{k}, j_{k}} x^{i_{k}} y^{j_{k}}\right)^{-1}\left[I+\sum_{\substack{p=\ell \\
m+n=p \\
(m, n) \succeq\left(i_{k+1}, j_{k+1}\right)}}^{\infty} B_{\left(i_{k+1}, j_{k+1}\right),(m, n)} x^{m} y^{n}\right] \\
=\left[I+\sum_{\alpha=1}^{\infty} H_{i_{k}, j_{k}}^{\alpha}\left(x^{i_{k}} y^{j_{k}}\right)^{\alpha}\right]\left[I+\sum_{\substack{p=\ell \\
m+n=p \\
(m, n) \succeq\left(i_{k+1}, j_{k+1}\right)}}^{\infty} B_{\left(i_{k+1}, j_{k+1}\right),(m, n)} x^{m} y^{n}\right]
\end{gathered}
$$

where $B_{\left(i_{k}, j_{k}\right),(0,0)}=I$ for all $(i, j) \in \mathbb{N}_{0} \times \mathbb{N}_{0}$, and $B_{\left(i_{k}, j_{k}\right),(m, n)}=O$, if $\left(i_{k}, j_{k}\right) \succ(m, n) \neq$ $(0,0)$. By comparing the coefficient of $x^{m} y^{n}$ in both sides of Equation (4.7), we discover that

$$
\begin{gathered}
B_{\left(i_{k+1}, j_{k+1}\right),(1,0)}=\cdots=B_{\left(i_{k+1}, j_{k+1}\right),\left(i_{k}, j_{k}\right)}=O \\
B_{\left(i_{k+1}, j_{k+1}\right),(m, n)}=B_{\left(i_{k}, j_{k}\right),(m, n)}-\sum_{\alpha=1}^{\left\lfloor\frac{m+n}{i_{k}+j_{k}}\right\rfloor} H_{i_{k}, j_{k}}^{\alpha} B_{\left(i_{k+1}, j_{k+1}\right),\left(m-\alpha i_{k}, n-\alpha j_{k}\right)} .
\end{gathered}
$$

If $(m, n)=\left(i_{k}, j_{k}\right)$, since $B_{\left(i_{k+1}, j_{k+1}\right),\left(i_{k}, j_{k}\right)}=O$, the above implies that

$$
H_{i_{k}, j_{k}}=B_{\left(i_{k}, j_{k}\right),\left(i_{k}, j_{k}\right)} \cdot
$$


Equation (4.10) shows the relationship between $H_{i_{k}, j_{k}}$ and $B_{\left(i_{k}, j_{k}\right),\left(i_{k}, j_{k}\right)}$. We use this relationship to rewrite Equation (4.7) as

$$
\begin{gathered}
I+\sum_{\substack{p=\ell \\
m+n=p \\
(m, n) \succeq\left(i_{k+1}, j_{k+1}\right)}}^{\infty} B_{\left(i_{k+1}, j_{k+1}\right),(m, n)} x^{m} y^{n}=\left(I-H_{i_{k}, j_{k}} x^{i_{k}} y^{i_{k}}\right)\left[I+\sum_{\substack{p=\ell \\
m+n=p,(m, n) \succeq\left(i_{k}, j_{k}\right)}}^{\infty} B_{\left(i_{k}, j_{k}\right),(m, n)} x^{m} y^{n}\right] \\
=\left[I-B_{\left(i_{k}, j_{k}\right),\left(i_{k}, j_{k}\right)} x^{i_{k}} y^{i_{k}}\right]\left[I+\sum_{\substack{p=\ell \\
m+n=p,(m, n) \succeq\left(i_{k}, j_{k}\right)}}^{\infty} B_{\left(i_{k}, j_{k}\right),(m, n)} x^{m} y^{n}\right] .
\end{gathered}
$$

By equating the coefficient of $x^{s} y^{t}$ on both sides of Equation (4.11), we find

$$
B_{\left(i_{k+1}, j_{k+1}\right),(s, t)}=B_{\left(i_{k}, j_{k}\right),(s, t)}-B_{\left(i_{k}, j_{k}\right),\left(i_{k}, j_{k}\right)} B_{\left(i_{k}, j_{k}\right),\left(s-i_{k}, t-j_{k}\right)},
$$

an equation we use to prove the MIPPE2 analog of Theorem 3.4.1.

Theorem 4.3.1. Let $\left(i_{k}, j_{k}\right) \in \mathbb{N}_{0} \times \mathbb{N}_{0} \backslash\{(0,0)\}$. Define $B_{\left(i_{k}, j_{k}\right),(0,0)}=I$ and $B_{\left(i_{k}, j_{k}\right),(m, n)}$ $=O$ for $(1,0) \preceq(m, n) \preceq\left(i_{k-1}, j_{k-1}\right)$. Assume that $B_{\left(i_{k}, j_{k}\right),(m, n)} \leq$ Ofor all $\left(i_{k}, j_{k}\right) \preceq(m, n)$. Then $B_{\left(i_{k+1}, j_{k+1}\right),(s, t)} \leq O$ whenever $\left(i_{k+1}, j_{k+1}\right) \preceq(s, t)$.

Proof. Rewrite Equation (4.12) as $B_{\left(i_{k+1}, j_{k+1}\right),(s, t)}=\beta+\gamma$, where $\beta:=B_{\left(i_{k}, j_{k}\right),(s, t)}$ and $\gamma=-B_{\left(i_{k}, j_{k}\right),\left(i_{k}, j_{k}\right)} B_{\left(i_{k}, j_{k}\right),\left(s-i_{k}, t-j_{k}\right)}$. By the hypothesis, since $B_{\left(i_{k}, j_{k}\right),(s, t)}$ is either a zero or a negative matrix, $\beta$ is a non-positive matrix. It remains to show that $\gamma$ is also a nonpositive matrix. By the hypothesis, it is a product of two non-positive matrices. Thus, $-B_{\left(i_{k}, j_{k}\right),\left(i_{k}, j_{k}\right)} B_{\left(i_{k}, j_{k}\right),\left(s-i_{k}, t-j_{k}\right)}$ is either a zero or a negative matrix.

Theorem 4.3.2. (Structure Property) Let $\left(i_{k}, j_{k}\right) \in \mathbb{N}_{0} \times \mathbb{N}_{0} \backslash\{(0,0)\}$. Then

$$
\begin{aligned}
B_{\left(i_{k+1}, j_{k+1}\right),(s, t)} & =\sum_{\eta}(-1)^{\tau(\phi(\eta))-1} B_{\left(i_{k}, j_{k}\right), \phi(\eta)} \\
& =\sum_{\eta}(-1)^{\tau(\phi(\eta))+1} B_{\left(i_{k}, j_{k}\right), \phi(\eta)},
\end{aligned}
$$

where the sum is over all $\phi(\eta)=\left(\left(i_{1}, j_{1}\right),\left(i_{2}, j_{2}\right)\right)$ such that $|\phi(\eta)|=(s, t)$ and $\left(i_{1}, j_{1}\right)=$ $\left(i_{k}, j_{k}\right)$. (Recall that $B_{\left(i_{k}, j_{k}\right), \phi(\eta)}=B_{\left(i_{k}, j_{k}\right),\left(i_{1}, j_{1}\right)} B_{\left(i_{k}, j_{k}\right),\left(i_{2}, j_{2}\right)} \ldots B_{\left(i_{k}, j_{k}\right),\left(i_{\tau}, j_{\tau}\right)}$.) If $B_{\left(i_{k}, j_{k}\right),(s, t)} \leq O$ for all $\left(i_{k}, j_{k}\right) \preceq(s, t)$, then Equation (4.13) is equivalent to

$$
B_{\left(i_{k+1}, j_{k+1}\right),(s, t)}=-\sum_{\eta}\left|B_{\left(i_{k}, j_{k}\right),\left(i_{1}, j_{1}\right)}\right| \ldots\left|B_{\left(i_{k}, j_{k}\right),\left(i_{\tau}, j_{\tau}\right)}\right|,
$$

where the range of summation is identical to the range of summation used in Equation (4.13). 
Proof. Rewrite Equation (4.12) as

$$
B_{\left(i_{k+1}, j_{k+1}\right),(s, t)}=\sum_{\substack{\alpha \in\{0,1\} \\ \alpha i_{j}+m=s \\ \alpha j_{k}+n=t}}(-1)^{\alpha} B_{\left(i_{k}, j_{k}\right),\left(i_{k}, j_{k}\right)}^{\alpha} B_{\left(i_{k}, j_{k}\right),\left(s-\alpha i_{k}, t-\alpha j_{k}\right)} .
$$

We obtain the desired result by representing $B_{\left(i_{k}, j_{k}\right),\left(i_{k}, j_{k}\right)}^{\alpha} B_{\left(i_{k}, j_{k}\right),(m, n)}$ in Equation (4.15) as $B_{\left(i_{k}, j_{k}\right), \phi(\eta)}$.

Let us see what will happen when we iterate Equation (4.14). In order to efficiently record the results, recall that $\phi=\left(\left(i_{1}, j_{1}\right),\left(i_{2}, j_{2}\right), \ldots,\left(i_{n}, j_{n}\right)\right)$ denotes a vector with $n$ components, (where $n \in \mathbb{N}$, and $i_{1}, i_{2}, \ldots, i_{n}, j_{1}, j_{2}, \ldots, j_{n} \in \mathbb{N}_{0}$ ), and $A_{\phi(\eta)}$ denotes the expression $A_{i_{1}, j_{1}} A_{i_{2}, j_{2}} \ldots A_{i_{n}, j_{n}}$. After $L$ iterations, and assuming $B_{\left(i_{k}, j_{k}\right),(s, t)} \leq O$, whenever $\left(i_{k}, j_{k}\right) \preceq(s, t)$, we obtain

$$
B_{\left(i_{k+1}, j_{k+1}\right),(s, t)}=\sum_{\eta}(-1)^{\tau(\phi(\eta))-1} A_{\phi(\eta)}=-\sum_{\eta}\left|A_{i_{1}, j_{1}}\right|\left|A_{i_{2}, j_{2}}\right| \cdots\left|A_{i_{\tau}, j_{\tau}}\right|,
$$

where the sum is over all $\phi(\eta)=\left(\left(i_{1}, j_{1}\right),\left(i_{2}, j_{2}\right), \ldots,\left(i_{\tau}, j_{\tau}\right)\right)$ such that $|\phi(\eta)|=(s, t)$.

If $(s, t)=\left(i_{k+1}, j_{k+1}\right)$, Equation (4.16) becomes

$$
\begin{aligned}
B_{\left(i_{k+1}, j_{k+1}\right),\left(i_{k+1}, j_{k+1}\right)}=H_{i_{k+1}, j_{k+1}} & =\sum_{\eta}(-1)^{\tau(\phi(\eta))-1} A_{\phi(\eta)} \\
& =-\sum_{\eta}\left|A_{i_{1}, j_{1}}\right|\left|A_{i_{2}, j_{2}}\right| \ldots\left|A_{i_{\tau}, j_{\tau}}\right|,
\end{aligned}
$$

where the sum is over all $\phi(\eta)=\left(\left(i_{1}, j_{1}\right),\left(i_{2}, j_{2}\right), \ldots,\left(i_{\tau}, j_{\tau}\right)\right)$ such that $|\phi(\eta)|=\left(i_{k+1}, j_{k+1}\right)$.

We now furnish an example to illustrate the structure property

\section{Example 4.3.1.}

$$
\begin{aligned}
H_{2,0} & =B_{(2,0),(2,0)} \\
& =B_{(0,1),(2,0)}-B_{(0,1),(0,1)} \underbrace{B_{(0,1),(2,-1)}}_{=0} \\
& =B_{(1,0),(2,0)}-B_{(1,0),(1,0)} B_{(1,0),(1,0)} \\
& =(-1)^{0} A_{2,0}+(-1)^{1} A_{1,0}^{2} .
\end{aligned}
$$

The explicit coefficients $H_{m, n}$ with $1 \leq m+n \leq 4$, as polynomials of $A_{1,0}, \ldots, A_{m, n}$, are given below.

$$
H_{1,0}=(-1)^{0} A_{1,0}
$$




$$
\begin{aligned}
H_{0,1}= & (-1)^{0} A_{0,1} \\
H_{2,0}= & (-1)^{0} A_{2,0}+(-1)^{1} A_{1,0}^{2} \\
H_{1,1}= & (-1)^{0} A_{1,1}+(-1)^{1} A_{1,0} A_{0,1} \\
H_{0,2}= & (-1)^{0} A_{0,2}+(-1)^{1} A_{0,1}^{2} \\
H_{3,0}= & (-1)^{0} A_{3,0}+(-1)^{1} A_{1,0} A_{2,0} \\
H_{2,1}= & (-1)^{0} A_{2,1}+(-1)^{1} A_{0,1} A_{2,0}+(-1)^{1} A_{1,0} A_{1,1}+(-1)^{2} A_{1,0}^{2} A_{0,1} \\
H_{1,2}= & (-1)^{0} A_{1,2}+(-1)^{1} A_{1,0} A_{0,2}+(-1)^{1} A_{0,1} A_{1,1}+(-1)^{2} A_{0,1} A_{1,0} A_{0,1} \\
H_{0,3}= & (-1)^{0} A_{0,3}+(-1)^{1} A_{0,1} A_{0,2} \\
H_{4,0}= & (-1)^{0} A_{4,0}+(-1)^{3} A_{1,0}^{4}+(-1)^{1} A_{2,0}^{2}+(-1)^{2} A_{1,0}^{2} A_{2,0}+(-1)^{2} A_{2,0} A_{1,0}^{2}+ \\
& (-1)^{1} A_{1,0} A_{3,0} \\
H_{3,1}= & (-1)^{0} A_{3,1}+(-1)^{1} A_{0,1} A_{3,0}+(-1)^{2} A_{0,1} A_{1,0} A_{2,0}+(-1)^{1} A_{1,0} A_{2,1}+ \\
& (-1)^{2} A_{1,0}^{2} A_{1,1}+(-1)^{3} A_{1,0}^{3} A_{0,1}+(-1)^{1} A_{2,0} A_{1,1}+(-1)^{2} A_{2,0} A_{1,0} A_{0,1} \\
H_{2,2}= & (-1)^{0} A_{2,2}+(-1)^{2} A_{1,0}^{2} A_{0,2}+(-1)^{2} A_{2,0} A_{0,1}^{2}+(-1)^{2} A_{1,1} A_{1,0} A_{0,1}+ \\
& (-1)^{1} A_{1,1}^{2}+(-1)^{2} A_{1,0} A_{0,1} A_{1,1}+(-1)^{2} A_{0,1} A_{1,0} A_{1,1}+ \\
& (-1)^{1} A_{1,0} A_{1,2}+(-1)^{1} A_{0,1} A_{2,1}+(-1)^{3} A_{0,1} A_{1,0}^{2} A_{0,1}+ \\
& (-1)^{2} A_{1,1} A_{1,0} A_{0,1}+(-1)^{3} A_{1,0} A_{0,1} A_{1,0} A_{0,1} \\
H_{1,3}= & (-1)^{0} A_{1,3}+(-1)^{1} A_{1,0} A_{0,3}+(-1)^{1} A_{0,1} A_{2,1}+(-1)^{2} A_{0,1}^{2} A_{1,1} \\
& +(-1)^{3} A_{0,1}^{2} A_{1,0} A_{0,1}+(-1)^{2} A_{0,1} A_{1,0} A_{0,2}+(-1)^{1} A_{1,1} A_{0,2}+ \\
& (-1)^{2} A_{1,0} A_{0,1} A_{0,2} \\
H_{0,4}= & (-1)^{0} A_{0,4}+(-1)^{3} A_{0,1}^{4}+(-1)^{1} A_{0,2}^{2}+(-1)^{2} A_{0,2} A_{0,1}^{2}+(-1)^{2} A_{0,1}^{2} A_{0,2}+ \\
& (-1)^{1} A_{0,1} A_{0,3} . \\
& (-1,0)
\end{aligned}
$$

\subsection{Convergence criteria for IMPPE2's}

The primary objective of this section is to present results concerning the convergence domain of the IMPPE2 in Equation (4.2). The convergence of an IMPPE2 is a subtle concept. In this chapter we define two notions of convergence. Both notions of convergence require converting the IMPPE2 into an MPPE2. The difference between these notions involves how this conversion takes place. For what we call "inverse" convergence, we use the domain of convergence of the inverse function which is an MPPE2. For what we call "primary" type of convergence, we first expand each elementary factor of the IMPPE2 via a geometric series expansion and then look at the ensuing MPPE2. In the case of one variable, the second author showed that both types of convergence provide the same 
estimate regarding a domain of absolute convergence; see Theorem 5.1 of [17]. However, for the case of two variables, as we shall soon discover, the estimate for the domain of absolute convergence provided by the notion of "inverse" convergence is larger than the estimate provided by the "primary" type of convergence; see Theorem 4.4.1. We begin with the definition of inverse convergence of an IMPPE2.

Definition 4.4.1. Given an IMPPE2 LTR $\prod_{\substack{q=1 \\ m+n=q}}^{\infty}\left(I-H_{m, n} x^{m} y^{n}\right)^{-1}$, we say the IMPPE2 convergence in the inverse sense if and only if $F(x, y)^{-1}=R T L \prod_{\substack{q=1 \\ m+n=q}}^{\infty}\left(I-H_{m, n} x^{m} y^{n}\right)$ converges, which means $\lim _{P \rightarrow \infty} R T L \prod_{\substack{q=1 \\ m+n=q}}^{P}\left(I-H_{m, n} x^{m} y^{n}\right)$ converges to a nonzero matrix. The order of the elementary factors within RTL $\prod_{\substack{q=1 \\ m+n=q}}^{P}\left(I-H_{m, n} x^{m} y^{n}\right)$ follows the conventions of Definition 1.2.1.

Definition 4.4.2. Given a suitable norm, an IMPPE2 LTR $\prod_{\substack{q=1 \\ m+n=q}}^{\infty}\left(I-H_{m, n} x^{m} y^{n}\right)^{-1}$ converges absolutely in the inverse sense if and only if $\prod_{\substack{q=1 \\ m+n=q}}^{\infty}\left(1+|| H_{m, n}||\left|x^{m} y^{n}\right|\right)$ converges to a nonzero real number, that is if and only if $\lim _{m, n \rightarrow \infty} \prod_{i=1}^{n} \prod_{j=1}^{m}\left(1+|| H_{i, j}||\left|x^{i} y^{j}\right|\right)$ converges to a nonzero real number.

Since $e^{x} \leq x+1$, the proof of Proposition 4.4.1 is directly transferrable and proves the following proposition:

Proposition 4.4.1. Given a suitable matrix norm, an IMPPE2 LTR $\prod_{\substack{q=1 \\ m+n=q}}^{\infty}\left(I-H_{m, n} x^{m} y^{n}\right)^{-1}$ is absolutely convergent in the inverse sense if and only if $\sum_{\substack{q=1 \\ m+n=q}}^{\infty} \| H_{m, n}||\left|x^{m} y^{n}\right|$ is an absolutely convergent series of real numbers.

Next we will define the notion of primary convergence.

Definition 4.4.3. We say the IMPPE2 LTR $\prod_{\substack{q=1 \\ m+n=q}}^{\infty}\left(I-H_{m, n} x^{m} y^{n}\right)^{-1}$ converges (in the primary sense) if the following two conditions hold: 
(1.) Each matrix function $\left(I-H_{m, n} x^{m} y^{n}\right)^{-1}$, where $(m, n) \in \mathbb{N}_{0} \times \mathbb{N}_{0} \backslash\{(0,0)\}$, is invertible, and

$$
\operatorname{LTR} \prod_{\substack{q=1 \\ m+n=q}}^{\infty}\left(I-H_{m, n} x^{m} y^{n}\right)^{-1}:=\operatorname{LTR} \prod_{\substack{q=1 \\ m+n=q}}^{\infty}\left(I+\sum_{\alpha=1}^{\infty} H_{m, n}^{\alpha} x^{\alpha m} y^{\alpha n}\right)
$$

converges in some suitable norm.

Condition (2.) of Definition 4.4.3 is equivalent to saying that $\lim _{P \rightarrow \infty} L T R \prod_{\substack{q=1 \\ m+n=q}}^{P}\left(I+\sum_{\alpha=1}^{\infty} H_{m, n}^{\alpha} x^{\alpha m} y^{\alpha n}\right)$ converges to a nonzero matrix.

Definition 4.4.4. Given a suitable matrix norm, we say the IMPPE2 $L T R \prod_{\substack{q=1 \\ m+n=q}}^{\infty}\left(I-H_{m, n} x^{m} y^{n}\right)^{-1}$ converges absolutely (in the primary sense) if the following two conditions hold:

(1.) Each matrix function $\left(I-H_{m, n} x^{m} y^{n}\right)^{-1}$, where $(m, n) \in \mathbb{N}_{0} \times \mathbb{N}_{0} \backslash\{(0,0)\}$, is invertible, and

(2.) $\prod_{\substack{q=1 \\ m+n=q}}^{\infty}\left(1+\left\|\sum_{\alpha=1}^{\infty} H_{m, n}^{\alpha} x^{\alpha m} y^{\alpha n}\right\|\right)$ converges to a nonzero real number.

Proposition 4.4.1, along with the monotonicity of the norm, shows that Condition (2.) of Definition 4.4.4 is equivalent to

$$
\text { (2'.) } \sum_{\substack{q=1 \\ m+n=q}}^{\infty} \sum_{\alpha=1}^{\infty}\left\|H_{m, n}\right\|^{\alpha}\left|x^{\alpha m} y^{\alpha n}\right| \text { being convergent. }
$$

If the coefficients $\left(H_{m, n}\right)$ of the IMPPE2 are commutative, (primary) convergence can be described in terms of the power series expansion of $\log \left(I-H_{m, n} x^{m} y^{n}\right)$. For any two commutative matrices $A, B \in M_{d}(\mathbb{C})$, it can be shown that $e^{A+B}=e^{A} e^{B}$ [[14], Proposition 2.5, P. 35]. Thus, if the coefficients $\left(H_{m, n}\right)$ of the IMPPE2 are commutative, we define

$$
\log \left(\operatorname{LTR} \prod_{\substack{q=1 \\ m+n=q}}^{\infty}\left(I-H_{m, n} x^{m} y^{n}\right)^{-1}\right):=-\sum_{\substack{q=1 \\ m+n=q}}^{\infty} \log \left(I-H_{m, n} x^{m} y^{n}\right),
$$

where

$$
\log \left(I-H_{m, n} x^{m} y^{n}\right):=-\sum_{\ell=1}^{\infty} \frac{H_{m, n}^{\ell}}{\ell} x^{m \ell} y^{n \ell}
$$


Equation (4.18) shows that

$$
\begin{aligned}
\exp \left(-\sum_{\substack{q=1 \\
m+n=q}}^{\infty} \log \left(I-H_{m, n} x^{m} y^{n}\right)\right) & :=\exp \left[\log \left(\operatorname{LTR} \prod_{\substack{q=1 \\
m+n=q}}^{\infty}\left(I-H_{m, n} x^{m} y^{n}\right)^{-1}\right)\right] \\
& =\operatorname{LTR} \prod_{\substack{q=1 \\
m+n=q}}^{\infty}\left(I-H_{m, n} x^{m} y^{n}\right)^{-1},
\end{aligned}
$$

and implies that for a sequence of commutative coefficients $\left(H_{m, n}\right)$,

$\operatorname{LTR} \prod_{\substack{q=1 \\ m+n=q}}^{\infty}\left(I-H_{m, n} x^{m} y^{n}\right)^{-1}$ converges to a nonzero matrix if and only if the double series

$$
-\sum_{\substack{q=1 \\ m+n=q}}^{\infty} \log \left(I-H_{m, n} x^{m} y^{n}\right)=\sum_{\substack{q=1 \\ m+n=q}}^{\infty} \sum_{\ell=1}^{\infty} \frac{H_{m, n}^{\ell}}{\ell} x^{m \ell} y^{n \ell}
$$

is convergent.

By adapting the Taylor series argument found on Page 165 of [5] we have

$$
1 / 2|| \Delta\|\leq\| \log (I+\Delta)\|\leq 3 / 2\| \Delta\|, \quad\| \Delta \|<1 / 2
$$

(In our case $\Delta=-H_{m, n} x^{m} y^{n}$.)

Equation (4.21) implies that $\sum_{\substack{q=1 \\ m+n=q}}^{\infty} \log \left(I-H_{m, n} x^{m} y^{n}\right)$ is absolutely convergent if and only if $\sum_{\substack{q=1 \\ m+n=q}}^{\infty} H_{m, n} x^{m} y^{n}$ is absolutely convergent. We summarize the previous discussion in the follow proposition.

Proposition 4.4.2. Let $\left(H_{m, n}\right)$ be a sequence of commutative matrices associated with the matrix function $F(x, y)=\operatorname{LTR} \prod_{\substack{q=1 \\ m+n=q}}^{\infty}\left(I-H_{m, n} x^{m} y^{n}\right)^{-1}$. Define

$$
\log F(x, y):=-\sum_{\substack{q=1 \\ m+n=q}}^{\infty} \log \left(I-H_{m, n} x^{m} y^{n}\right)=\sum_{\substack{q=1 \\ m+n=q}}^{\infty} \sum_{\ell=1}^{\infty} \frac{H_{m, n}^{\ell}}{\ell} x^{m \ell} y^{n \ell}
$$

The IMPEE2 of $F(x, y), \operatorname{LTR} \prod_{\substack{q=1 \\ m+n=q}}^{\infty}\left(I-H_{m, n} x^{m} y^{n}\right)^{-1}$, converges to a nonzero value if and only $\log F(x, y)=\sum_{\substack{q=1 \\ m+n=q}}^{\infty} \log \left(I-H_{m, n} x^{m} y^{n}\right)$ converges. The IMPEE2 of $F(x, y)$, LTR $\prod_{\substack{q=1 \\ m+n=q}}^{\infty}(I-$ 
$\left.H_{m, n} x^{m} y^{n}\right)^{-1}$, is absolutely convergent if and only if $\sum_{\substack{q=1 \\ m+n=q}}^{\infty} \log \left(I-H_{m, n} x^{m} y^{n}\right)$ is absolutely convergent, namely if and only if $\sum_{\substack{q=1 \\ m+n=q}}^{\infty}\left\|\sum_{\ell=1}^{\infty} \frac{H_{m, n}^{\ell}}{\ell} x^{m \ell} y^{n \ell}\right\|<\infty$.

The following remark emphasizes the connection inverse convergence, primary convergence, and the domain of convergence determined by the logarithm of a two variable matrix function.

Remark 4.4.1. Let

$$
\begin{aligned}
& \mathbb{D}_{1}=\left\{(x, y) \in \mathbb{C}^{2}:|x|<\rho_{1} \text { and }|y|<\rho_{1}\right\} \\
& \mathbb{D}_{2}=\left\{(x, y) \in \mathbb{C}^{2}:|x|<\rho_{2} \text { and }|y|<\rho_{2}\right\}
\end{aligned}
$$

be the domains of convergence of the matrices functions $M(x, y)$ and $M(x, y)^{-1}$, respectively. Then $\mathbb{D}$ the domain of convergence of $\log M(x, y)$, satisfies

$$
\mathbb{D}=\left\{(x, y) \in \mathbb{C}^{2}:|x|<\rho \text { and }|y|<\rho\right\},
$$

where $\rho=\min \left\{\rho_{1}, \rho_{2}\right\}$.

The first major result of this section provides a lower bound for the domain of convergence an IMPPE2 in terms a majorizing log series.

Theorem 4.4.1. (1.) Given a power series of a matrix function $F(x, y)$

$$
F(x, y)=I+\sum_{\substack{p=1 \\ m+n=p}}^{\infty} A_{m, n} x^{m} y^{n}=\operatorname{LTR} \prod_{\substack{q=1 \\ m+n=q}}^{\infty}\left(I-H_{m, n} x^{m} y^{n}\right)^{-1} .
$$

where $A_{m, n}, H_{m, n} \in M_{d}(\mathbb{C})$, define the following auxiliary matrices functions with coefficients over $M_{d}(\mathbb{R})$ :

$$
\begin{array}{r}
C(x, y)=I-\sum_{\substack{p=1 \\
m+n=p}}^{\infty}\left|A_{m, n}\right| x^{m} y^{n}=L T R \prod_{\substack{q=1 \\
m+n=q}}^{\infty}\left(I+S_{m, n} x^{m} y^{n}\right)^{-1} \\
M(x, y)=I-\sum_{\substack{p=1 \\
m+n=p}}^{\infty} M_{m, n} x^{m} y^{n}=L T R \prod_{\substack{q=1 \\
m+n=q}}^{\infty}\left(I+R_{m, n} x^{m} y^{n}\right)^{-1} . \\
\text { If }\left|A_{m, n}\right| \leq M_{m, n} \text { for all }(m, n) \in \mathbb{N}_{0} \times \mathbb{N}_{0} \backslash\{(0,0)\}, \text { then } \\
O \leq\left|H_{m, n}\right| \leq S_{m, n} \leq R_{m, n} \text { for all }(m, n) \in \mathbb{N}_{0} \times \mathbb{N}_{0} \backslash\{(0,0)\} .
\end{array}
$$


(2.) Define $W \in M_{d}(\mathbb{C})$ as

$$
W=\left(w_{u, v}\right), \quad \text { where } \quad w_{u, v}=1, \quad u, v=1, \ldots, d .
$$

Furthermore, given $A_{m, n} \in M_{d}(\mathbb{C})$ where $A_{m, n}=\left(a_{\psi, \omega}(m, n)\right)$ for $\psi, \omega=1, \ldots, d$, define

$$
a(m, n):=\max _{\psi, \omega}\left|a_{\psi, \omega}(m, n)\right|, \quad \text { and } \quad s:=\sup _{m+n \geq 1}[a(m, n)]^{\frac{1}{m+n}}
$$

Consider a special case for $M(x, y)$, namely

$$
\begin{aligned}
M(x, y) & =I-\sum_{\substack{p=1 \\
m+n=p}}^{\infty} s^{m+n} W x^{m} y^{n}=I-W\left[\sum_{m=0}^{\infty}(s x)^{m} \sum_{n=0}^{\infty}(s y)^{n}-1\right] \\
& =I-\left[\frac{1}{(1-s x)(1-s y)}-1\right] W=I-\left[\frac{s(x+y)-s^{2} x y}{(1-s x)(1-s y)}\right] W \\
& =\frac{I-s(W+I)(x+y)+s^{2}(W+I) x y}{(1-s x)(1-s y)} \\
& =L T R \prod_{\substack{q=1 \\
m+n=q}}^{\infty}\left(I+R_{m, n} x^{m} y^{n}\right)^{-1},
\end{aligned}
$$

where $M_{m, n}=s^{m+n} W$. Since $\left(s^{m+n} W\right)$ is a sequence of commutative matrices, $\left(R_{m, n}\right)$ is also a sequence of commutative matrices, and Equation (4.18) is applicable. Thus, by using the Taylor series of expansion $\log \left(I+R_{m, n} x^{m} y^{n}\right)$, we define

$$
\begin{aligned}
\log (M(x, y)) & =\log \left(\operatorname{LTR} \prod_{\substack{q=1 \\
m+n=q}}^{\infty}\left(I+R_{m, n} x^{m} y^{n}\right)^{-1}\right) \\
& =-\sum_{\substack{q=1 \\
m+n=q}}^{\infty} \log \left(I+R_{m, n} x^{m} y^{n}\right)=\sum_{\substack{q=1 \\
m+n=q}}^{\infty} \sum_{\ell=1}^{\infty} \frac{(-1)^{\ell} R_{m, n}^{\ell}\left(x^{m} y^{n}\right)^{\ell}}{\ell} \\
& =\sum_{\substack{p=1 \\
m+n=p}}^{\infty} P_{m, n} x^{m} y^{n}, \quad \text { where } P_{m, n}:=\sum_{\substack{\ell \mid d \\
d=\operatorname{gcd}(m, n)}} \frac{(-1)^{\ell}\left(R_{\frac{m}{\ell}, \frac{n}{\ell}}\right)^{\ell}}{\ell} .
\end{aligned}
$$

Let $\|\cdot\|$ be a suitable norm and $\rho(\|\cdot\|)$ be the radius of convergence of 


$$
\begin{aligned}
& \sum_{\substack{p=1 \\
m+n=p}}^{\infty} P_{m, n} x^{m} y^{n} \text { as defined via Equation (4.27). Then } \\
& \quad R_{m, n}=E_{m, n} \leq-P_{m, n}, \quad \text { if } m=2 \ell+1 \text { or } n=2 \ell+1 \text { for } \ell \in \mathbb{N}, \\
& \quad \text { where } E_{m, n} \text { is given in Theorem 3.5.1. }
\end{aligned}
$$

The IMPPE2 LTR $\prod_{\substack{q=1 \\ m+n=q}}^{\infty}\left(I-H_{m, n} x^{m} y^{n}\right)^{-1}$ converges absolutely for $\rho(\|\cdot\|)$ in the sense of inverse convergence (see Definition 4.4.2) over the domain

$$
\mathbb{D}_{1}=\left\{(x, y) \in \mathbb{C}^{2}:(d+1) s(|x|+|y|)+(d+1) s^{2}|x| y \mid<1\right\} .
$$

Furthermore, the matrices $F(x, y), C(x, y), M(x, y)$ are invertible in $\mathbb{D}_{1}$, and their inverses

$$
\begin{aligned}
& F(x, y)^{-1}=R T L \prod_{\substack{q=1 \\
m+n=q}}^{\infty}\left(I-H_{m, n} x^{m} y^{n}\right), C(x, y)^{-1}=R T L \prod_{\substack{q=1 \\
m+n=q}}^{\infty}\left(I+S_{m, n} x^{m} y^{n}\right) \\
& M(x, y)^{-1}=R T L \prod_{\substack{q=1 \\
m+n=q}}^{\infty}\left(I+R_{m, n} x^{m} y^{n}\right),
\end{aligned}
$$

are absolutely convergent in $\mathbb{D}_{1}$.

(3.) Define $\hat{M}(x, y)$, a majorant of $M(x, y)$, as follows:

$$
\hat{M}(x, y)=I-W \sum_{q=1}^{\infty}(s x+s y)^{q}=\prod_{\substack{q=1 \\ m+n=q}}^{\infty}\left(I+\hat{R}_{m, n} x^{m} y^{n}\right)^{-1} .
$$

The IMPPE2's LTR $\prod_{\substack{q=1 \\ m+n=q}}^{\infty}\left(I-H_{m, n} x^{m} y^{n}\right)^{-1}, \operatorname{LTR} \prod_{\substack{q=1 \\ m+n=q}}^{\infty}\left(I+S_{m, n} x^{m} y^{n}\right)^{-1}$,

$\operatorname{LTR} \prod_{\substack{q=1 \\ m+n=q}}^{\infty}\left(I+R_{m, n} x^{m} y^{n}\right)^{-1}$ possess absolutely convergent infinite product representations (in the sense of Definition 4.4.4) in the polydiscs

$$
\mathbb{D}_{2}=\left\{(x, y)^{2} \in \mathbb{C}:|x|<\frac{1}{2 s(d+1)} \text { and }|y|<\frac{1}{2 s(d+1)}\right\}
$$


Proof.(1.) Equation (4.17) implies that

$$
H_{m, n}=\sum_{\substack{\eta \\|\phi(\eta)|=(m, n)}}(-1)^{\tau(\phi(\eta))+1} A_{\phi(\eta)}=\sum_{\substack{\eta \\|\phi(\eta)|=(m, n)}}(-1)^{\tau(\phi(\eta))+1} A_{i_{1}, j_{1}} \ldots A_{i_{\tau}, j_{\tau}},
$$

which in turn implies that

$$
\left|H_{m, n}\right|=\left|\sum_{\substack{\eta \\|\phi(\eta)|=(m, n)}}(-1)^{\tau(\phi(\eta))-1} A_{i_{1}, j_{1}} \ldots A_{i_{\tau}, j_{\tau}}\right| \leq \sum_{|\phi(\eta)|=(m, n)}\left|A_{i_{1}, j_{1}}\right| \ldots\left|A_{i_{\tau}, j_{\tau}}\right| .
$$

Similarly, when we apply Equation (4.17) to Equation (4.23), we obtain

$$
\begin{aligned}
O \leq S_{m, n} & =\sum_{\substack{\eta \\
|\phi(\eta)|=(m, n)}}(-1)^{\tau(\phi(\eta))}\left(-\left|A_{i_{1}, j_{1}}\right|\right) \ldots\left(-\left|A_{i_{\tau}, j_{\tau}}\right|\right) \\
& =\sum_{\eta}(-1)^{\tau(2 \phi(\eta))}\left(\left|A_{i_{1}, j_{1}}\right|\right) \ldots\left(\left|A_{i_{\tau}, j_{\tau}}\right|\right) \\
& =\sum_{\substack{\eta(\eta)|=(m, n)\\
| \phi(\eta) \mid=(m, n)}}\left(\left|A_{i_{1}, j_{1}}\right|\right) \ldots\left(\left|A_{i_{\tau}, j_{\tau}}\right|\right) .
\end{aligned}
$$

Combining Equations (4.32) and (4.33), we deduce that $\left|H_{m, n}\right| \leq S_{m, n}$. Also, due to the inequality $\left|A_{m, n}\right| \leq M_{m, n}$, then we have

$$
\begin{aligned}
O \leq S_{m, n} & =\sum_{\substack{\eta \\
|\phi(\eta)|=(m, n)}}\left|A_{i_{1}, j_{1}}\right| \ldots\left|A_{i_{\tau}, j_{\tau}}\right| \\
& \leq \sum_{\substack{\eta \\
|\phi(\eta)|=(m, n)}} M_{i_{1}, j_{1}} \ldots M_{i_{\tau}, j_{\tau}}=R_{m, n},
\end{aligned}
$$

where the last equality follows from Equation (4.17). Hence, $O \leq\left|H_{m, n}\right| \leq S_{m, n} \leq R_{m, n}$ and (4.25) is proven.

(2.) By the definition of $P_{m, n}$ provided via (4.27), we see that

$$
P_{m, n}=\sum_{\substack{\ell \mid d \\
d=g c d(m, n)}} \frac{(-1)^{\ell} R_{m, n}}{\ell}=-R_{m, n}+\sum_{\begin{array}{c}
\ell \mid d, \ell \neq 1 \\
d=g c d(m, n)
\end{array}} \frac{(-1)^{\ell}\left(R_{\frac{m}{\ell}, \frac{n}{\ell}}\right)^{\ell}}{\ell},
$$

and since Part (1.) implies that $R_{m, n} \geq O$, the preceding equation implies that

$$
O \leq R_{m, n} \leq-P_{m, n}, \quad \text { if } m \text { or } n \text { is an odd integer. }
$$

Moreover, by Theorem 2.2.1, we know that the coefficients $E_{m, n}=R_{m, n}$ if $m$ or $n$ is an odd integer, which completes the proof of (4.28). 
To obtain the domain of inverse convergence as provided by (4.29), we first calculate the characteristic polynomial of the matrix $W$ as

$$
\operatorname{det}(x I-W)=(x-d) x^{d-1} .
$$

Then a straightforward computation in Equation (4.26) shows that eigenvalues of $M(x, y)$ are 1 and $\frac{1-s(d+1)(x+y)+s^{2}(d+1) x y}{(1-s x)(1-s y)}$. As a result, the eigenvalues of $M(x, y)^{-1}$ are 1 and $\frac{(1-s x)(1-s y)}{1-s(d+1)(x+y)+s^{2}(d+1) x y}$. Note that $\frac{(1-s x)(1-s y)}{1-s(d+1)(x+y)+s^{2}(d+1) x y}=0$ or $\infty$ if and only if $s x=1$ or $s y=1$, or $1-s(d+1)(x+y)+s^{2}(d+1) x y=0$. Thus the nearest singularities of $M(x, y)^{-1}$ to $(x, y)=(0,0)$ are the the points $(x, y)$ satisfy the equation $1-s(d+1)(x+$ $y)+s^{2}(d+1) x y=0$, and the conclusion of (4.29) follows. What this argument shows is that $M(x, y)^{-1}=R T L \prod_{\substack{q=1 \\ m+n=q}}^{\infty}\left(I+R_{m, n} x^{m} y^{n}\right)$ is absolutely convergent (in the inverse sense) in $\mathbb{D}_{1}$. Since $O \leq\left|H_{m, n}\right| \leq S_{m, n} \leq R_{m, n}$, Proposition 4.4.1 implies that $F(x, y)^{-1}$ and $C(x, y)^{-1}$ are also well defined and absolutely convergent over domain $\mathbb{D}_{1}$. See Figure 4.1.

(3.) In order to investigate the convergence of $L T R \prod_{\substack{q=1 \\ m+n=q}}^{\infty}\left(I-H_{m, n} x^{m} y^{n}\right)^{-1}$ in the sense of Definition 4.4.4), we define a majorant of $M(x, y)$, namely

$$
\begin{aligned}
\hat{M}(x, y) & =I-W \sum_{q=1}^{\infty}(s x+s y)^{q} \\
& =I-W \sum_{q=1}^{\infty} \sum_{k=0}^{q}\left(\begin{array}{l}
q \\
k
\end{array}\right)(s x)^{q-k}(s y)^{k} \quad \text { Set } m=q-k \text { and } n=k, \\
& =I-W \sum_{\substack{p=1 \\
m+n=p}}^{\infty}\left(\begin{array}{c}
m+n \\
n
\end{array}\right) s^{m+n} x^{m} y^{n}=I-\sum_{\substack{p=1 \\
m+n=p}}^{\infty} \hat{M}_{m, n} x^{m} y^{n},
\end{aligned}
$$

where $\hat{M}_{m, n}=W\left(\begin{array}{c}m+n \\ n\end{array}\right) s^{m+n}$.

Since $M_{m, n} \leq \hat{M}_{m, n}$, by the structure property we have

$$
O \leq\left|H_{m, n}\right| \leq R_{m, n} \leq \hat{R}_{m, n}
$$

Next we invert $\hat{M}(x, y)$ since this inversion will provide an upper bound for $\hat{R}_{m, n}$. In particular, we have

$$
[\hat{M}(x, y)]^{-1}=I+\sum_{\substack{p=1 \\ m+n=p}}^{\infty} \tilde{M}_{m, n} x^{m} y^{n}=R T L \prod_{\substack{p=1 \\ m+n=p}}^{\infty}\left(I+\hat{R}_{m, n} x^{m} y^{n}\right) .
$$


Since $\left(\hat{R}_{m, n}\right)_{m, n=0}^{\infty}$ is a sequence of positive matrices, both the MPPE2

$R T L \prod_{\substack{p=1 \\ m+n=p}}^{\infty}\left(I+\hat{R}_{m, n} x^{m} y^{n}\right)$ and its associated power series $I+\sum_{\substack{p=1 \\ m+n=p}}^{\infty} \tilde{M}_{m, n} x^{m} y^{n}$ have the same domain of convergence [see Corollary 6.1, [7] ]. Furthermore, coefficient comparison shows that

$$
O \leq \hat{R}_{m, n} \leq \tilde{M}_{m, n}
$$

Combining Equations (4.25), (4.35), and (4.36) together implies that

$$
\begin{aligned}
\left|\sum_{\substack{p=1 \\
m+n=p}}^{\infty} \sum_{k=1}^{\infty}\left(H_{m, n} x^{m} y^{n}\right)^{k}\right| & \leq \sum_{\substack{p=1 \\
m+n=p}}^{\infty} \sum_{k=1}^{\infty}\left|H_{m, n}\right|^{k}|x|^{m k}|y|^{n k} \\
& \leq \sum_{\substack{p=1 \\
m+n=p}}^{\infty} \sum_{k=1}^{\infty}\left(\hat{R}_{m, n}\right)^{k}|x|^{m k}|y|^{n k} \\
& \leq \sum_{\substack{p=1 \\
m+n=p}}^{\infty} \sum_{k=1}^{\infty}\left(\tilde{M}_{m, n}\right)^{k}|x|^{m k}|y|^{n k} .
\end{aligned}
$$

Equation (4.37) shows that we need to determine an upper bound for $\tilde{M}_{m, n}$. To find the desired upper bound, recall from (4.34) that

$$
\begin{aligned}
\hat{M}(x, y) & =I-W \sum_{q=1}^{\infty}(s x+s y)^{q}=I-\frac{W(s x+s y)}{1-(s x+s y)} \\
& =I-\sum_{\substack{p=1 \\
m+n=p}}^{\infty} \hat{M}_{m, n} x^{m} y^{n}, \quad \text { where } \hat{M}_{m, n}=W\left(\begin{array}{c}
m+n \\
n
\end{array}\right) s^{m+n} .
\end{aligned}
$$

To make the calculations easier set $z=x+y$ and get

$$
\hat{M}(z)=I-\frac{s z}{1-s z} W .
$$

We will now assume that $|s z|<1$ and $\frac{d|s z|}{1-|s z|} \leq \rho<1$. Then we find that

$$
\begin{array}{rlr}
\hat{M}^{-1}(z) & =\left[I-\frac{s z}{1-s z} W\right]^{-1}=I+\sum_{n=1}^{\infty}\left[\frac{s z}{1-s z}\right]^{n} W^{n} \\
& =I+\frac{W}{d} \sum_{n=1}^{\infty}\left[\frac{d s z}{1-s z}\right]^{n} & \text { Since } W^{n}=d^{n-1} W \\
& =I+\frac{s z W}{1-s z}\left[\frac{1}{1-\left(\frac{d s z}{1-s z}\right)}\right]=I+\frac{s z W}{1-(d+1) s z} &
\end{array}
$$




$$
=I+s z W \sum_{k=0}^{\infty}[(d+1) s z]^{k}=I+W \sum_{k=1}^{\infty}(d+1)^{k-1} s^{k} z^{k} .
$$

In Equation (4.39), we set $z=x+y$ to obtain

$$
\begin{aligned}
& {[\hat{M}(x, y)]^{-1}=I+W \sum_{k=1}^{\infty}(d+1)^{k-1} s^{k}(x+y)^{k}} \\
& =I+W \sum_{k=1}^{\infty}(d+1)^{k-1} s^{k} \sum_{p=0}^{k}\left(\begin{array}{l}
k \\
p
\end{array}\right) x^{p} y^{k-p} \\
& =I+W \sum_{k=1}^{\infty}(d+1)^{k-1} s^{k}\left[y^{k}+\sum_{p=1}^{k}\left(\begin{array}{l}
k \\
p
\end{array}\right) x^{p} y^{k-p}\right] \\
& =I+W \sum_{k=1}^{\infty}(d+1)^{k-1} s^{k} y^{k}+W \sum_{k=1}^{\infty}(d+1)^{k-1} s^{k} \sum_{p=1}^{k}\left(\begin{array}{l}
k \\
p
\end{array}\right) x^{p} y^{k-p} \\
& =I+W \sum_{k=1}^{\infty}(d+1)^{k-1} s^{k} y^{k}+W \sum_{p=1}^{\infty} \sum_{k=p}^{\infty}(d+1)^{k-1}\left(\begin{array}{l}
k \\
p
\end{array}\right) s^{k} x^{p} y^{k-p} \\
& =I+W \sum_{\substack{p=1 \\
m+n=1}}^{\infty}(d+1)^{m+n-1} s^{m+n}\left(\begin{array}{c}
m+n \\
n
\end{array}\right) x^{m} y^{n}, \quad \text { set } p=m \text { and } k=m+n \\
& =I+W \sum_{\substack{p=1 \\
m+n=1}}^{\infty} \tilde{M}_{m, n} x^{m} y^{n}
\end{aligned}
$$

where $\tilde{M}_{m, n}=(d+1)^{m+n-1} s^{m+n}\left(\begin{array}{c}m+n \\ n\end{array}\right) W$.

So, we have

$$
\tilde{M}_{m, n}=(d+1)^{m+n-1} s^{m+n}\left(\begin{array}{c}
m+n \\
n
\end{array}\right) W \leq(d+1)^{m+n-1}(2 s)^{m+n} W,
$$

where for the last inequality we made use of the fact that the sum of all the entries in the $m+n$-th row of Pascal's triangle is equal to $2^{m+n}$.

Plugging the desired upper bound of (4.41) into (4.37), we find that

$$
\begin{aligned}
& \left|\sum_{\substack{p=1 \\
m+n=p}}^{\infty} \sum_{k=1}^{\infty}\left(H_{m, n} x^{m} y^{n}\right)^{k}\right| \leq \sum_{\substack{p=1 \\
m+n=p}}^{\infty} \sum_{k=1}^{\infty}\left(\tilde{M}_{m, n}\right)^{k}|x|^{m k}|y|^{n k} \\
& \leq \sum_{\substack{p=1 \\
m+n=p}}^{\infty} \sum_{k=1}^{\infty} \frac{[2(d+1) s]^{k(m+n)}}{(d+1)^{k}} W^{k}|x|^{m k}|y|^{n k}
\end{aligned}
$$




$$
\begin{aligned}
& \leq \sum_{\substack{p=1 \\
m+n=p}}^{\infty} \sum_{k=1}^{\infty} \frac{[2(d+1) s]^{k(m+n)}}{(d+1)^{k}}\left[d^{k-1} W\right]|x|^{m k}|y|^{n k}, \quad \text { since } W^{k}=d^{k-1} W \\
& \leq W \sum_{\substack{p=1 \\
m+n=p}}^{\infty} \sum_{k=1}^{\infty} \frac{[2(d+1) s]^{k(m+n)}}{(d+1)^{k}}(d+1)^{k-1}|x|^{m k}|y|^{n k} \\
& \leq \frac{W}{(d+1)} \sum_{\substack{p=1 \\
m+n=p}}^{\infty} \sum_{k=1}^{\infty}\left[(2 s)^{m+n}(d+1)^{m+n}|x|^{m}|y|^{n}\right]^{k} \\
& =\frac{W}{(d+1)} \sum_{\substack{p=1 \\
m+n=p}}^{\infty} \frac{(2 s)^{m+n}(d+1)^{m+n}|x|^{m}|y|^{n}}{1-[2 s(d+1)|x|]^{m}[2 s(d+1)|y|]^{n}}
\end{aligned}
$$

where in the penultimate line we assumed $\left|(2 s)^{m+n}(d+1)^{m+n} x^{m} y^{n}\right|<1$. This assumption provides a further refinement on the polydiscs used in the computation of (4.39) where we assumed that $s(d+1)|x|<\rho<1$ and $s(d+1)|y|<\rho<1$. Now we have to shrink these polydiscs and require that $2 s(d+1)|x|<\rho_{1}<\rho<1$ and that $2 s(d+1)|y|<\rho_{2}<\rho<1$. When working over these refined polydiscs, the previous inequity becomes

$$
\begin{aligned}
& \left|\sum_{\substack{p=1 \\
m+n=p}}^{\infty} \sum_{k=1}^{\infty}\left(H_{m, n} x^{m} y^{n}\right)^{k}\right| \leq \frac{W}{(d+1)} \sum_{\substack{p=1 \\
m+n=p}}^{\infty} \frac{[2 s(d+1)|x|]^{m}[2 s(d+1)|y|]^{n}}{1-\rho^{2}} \\
& \leq \frac{W}{(d+1)(1-\rho)} \sum_{\substack{p=1 \\
m+n=p}}^{\infty}[2 s(d+1)|x|]^{m}[2 s(d+1)|y|]^{n} \quad \text { Since } \rho^{2}<\rho<1 \\
& \leq \frac{W}{(d+1)(1-\rho)} \sum_{\substack{p=1 \\
m+n=p}}^{\infty} \rho^{m+n} \\
& =\frac{W}{(d+1)(1-\rho)}\left[\sum_{m=0}^{\infty} \rho^{m} \sum_{n=0}^{\infty} \rho^{n}-1\right] \\
& =\frac{W}{(d+1)(1-\rho)}\left[\frac{1}{(1-\rho)^{2}}-1\right] \\
& =\frac{\left[2 \rho-\rho^{2}\right] W}{(d+1)(1-\rho)^{3}} .
\end{aligned}
$$

In conclusion, by using a majorant of a majorant and clever use of inversion, we finally 
showed

$$
\begin{aligned}
\left|\sum_{\substack{p=1 \\
m+n=p}}^{\infty} \sum_{k=1}^{\infty}\left(H_{m, n} x^{m} y^{n}\right)^{k}\right| & \leq \sum_{\substack{p=1 \\
m+n=p}}^{\infty} \sum_{k=1}^{\infty}\left(\tilde{M}_{m, n}\right)^{k}|x|^{m k}|y|^{n k} \\
& =\frac{\left[2 \rho-\rho^{2}\right] W}{(d+1)(1-\rho)^{3}},
\end{aligned}
$$

whenever $2 s(d+1)|x|<\rho<1$ and $2 s(d+1)|y|<\rho<1$.

Therefore, an estimate for domain of absolute convergence of $L T R \prod_{\substack{q=1 \\ m+n=q}}^{\infty}\left(I+\hat{R}_{m, n} x^{m} y^{n}\right)^{-1}$ is

$$
\mathbb{D}_{2}=\left\{(x, y) \in \mathbb{C}^{2}:|x|<\frac{1}{2 s(d+1)} \text { and }|y|<\frac{1}{2 s(d+1)}\right\} .
$$

Since $O \leq\left|H_{m, n}\right| \leq S_{m, n} \leq R_{m, n} \leq \hat{R}_{m, n}$, Definition 4.4.4 implies that $\mathbb{D}_{2}$ is also an estimate for the domain of absolute convergence of $\operatorname{LTR} \prod_{\substack{q=1 \\ m+n=q}}^{\infty}\left(I-H_{m, n} x^{m} y^{n}\right)^{-1}$ and of $\operatorname{LTR} \prod_{\substack{q=1 \\ m+n=q}}^{\infty}\left(I+S_{m, n} x^{m} y^{n}\right)^{-1}$

The inequality defining $\mathbb{D}_{1}$ makes it possible to obtain a domain of absolute convergence (in the inverse sense) in terms of polydiscs. Let $|x|<\rho^{\prime}$ and $|y|<\rho^{\prime}$. Then the inequality defining $\mathbb{D}_{1}$ implies that the IMPPE2 will be absolutely convergent if

$$
s(d+1)\left(2 \rho^{\prime}\right)+s^{2}(d+1)\left(\rho^{\prime}\right)^{2}=(d+1)\left[\left(s \rho^{\prime}+1\right)^{2}-1\right]<1,
$$

or equivalently, if $\rho^{\prime}<s^{-1}\left[\sqrt{\frac{d+2}{d+1}}-1\right]$. The inequalities for $\rho^{\prime}$ obtained from the defining quadratic equation of $\mathbb{D}_{1}$, namely

$$
|x|<s^{-1}\left[\sqrt{\frac{d+2}{d+1}}-1\right], \quad|y|<s^{-1}\left[\sqrt{\frac{d+2}{d+1}}-1\right]
$$

are sharp in the sense that if

$$
x=-s^{-1}\left[\sqrt{\frac{d+2}{d+1}}-1\right], \quad y=-s^{-1}\left[\sqrt{\frac{d+2}{d+1}}-1\right],
$$

then $\left|s(d+1)(x+y)-s^{2}(d+1) x y\right|=1$ and the sum of the absolute values of the terms in the power series of $M(x, y)^{-1}$ as defined in (4.30) diverge.

We can summarize what we have shown so far regrading the absolute convergence of IMPPE2 in both Equations (4.22), (4.26) in term of spectral conditions and obtain the second major result of this section. 
Theorem 4.4.2. (1.) Let $F(x, y)=I+\sum_{\substack{p=1 \\ m+n=p}}^{\infty} A_{m, n} x^{m} y^{n}$. Let $W$ and $s$ be as defined in Part (2.) of Theorem 4.4.1. Both $F(x, y)$ and its IMPPE2,

$$
F(x, y)=I+\sum_{\substack{p=1 \\ m+n=p}}^{\infty} A_{m, n} x^{m} y^{n}=\operatorname{LTR} \prod_{\substack{q=1 \\ m+n=q}}^{\infty}\left(I-H_{m, n} x^{m} y^{n}\right)^{-1},
$$

and the auxiliary function, along with its IMPPE2,

$$
M(x, y)=I-\sum_{\substack{p=1 \\ m+n=p}}^{\infty} s^{m+n} W x^{m} y^{n}=L T R \prod_{\substack{q=1 \\ m+n=q}}^{\infty}\left(I+R_{m, n} x^{m} y^{n}\right)^{-1}
$$

will be absolutely convergent in the sense of an inverse convergence whenever $(x, y) \in \mathbb{D}_{1}$, where

$$
\mathbb{D}_{1}=\left\{(x, y) \in \mathbb{C}^{2}:(d+1) s(|x|+|y|)+(d+1) s^{2}|x||y|<1\right\},
$$

See Figure 4.1, and in the sense of Definition 4.4 .4 in the polydisc

$$
\mathbb{D}_{2}=\left\{(x, y) \in \mathbb{C}^{2}:|x|<\frac{1}{2 s(d+1)} \text { and }|y|<\frac{1}{2 s(d+1)}\right\} .
$$

(2.) With the same conventions as in Part (1.), both $F(x, y)$ and its IMPPE2, along with $M(x, y)$ and its IMPPE2, will be absolutely convergent whenever $(x, y) \in D_{x \rho^{\prime}} \times$ $D_{y \rho^{\prime}}$ with $\rho^{\prime}<s^{-1}\left[\sqrt{\frac{d+2}{d+1}}-1\right]$, where

$$
D_{x \rho^{\prime}}: \equiv\left\{x:|x|<\rho^{\prime}\right\}, \quad D_{y \rho^{\prime}}: \equiv\left\{y:|y|<\rho^{\prime}\right\} .
$$




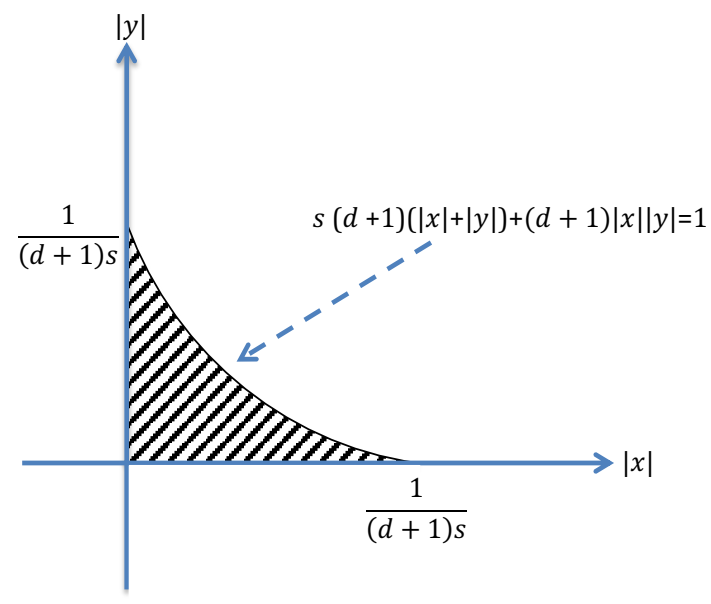

Figure 4.1: A domain of absolute convergence of Equations (4.22) and (4.24).

We should mention that for $F(x, y)=I+\sum_{\substack{p=1 \\ m+n=p}}^{\infty} A_{m, n} x^{m} y^{n}$, the results of Theorem 4.4.1(2) and Theorem 4.4.2 and can be stated and analogously proven with $s$ replaced by

$$
S:=\sup _{\substack{p=1 \\ m+n=p}}\left\|A_{m, n}\right\|^{\frac{1}{m+n}} .
$$

Furthermore, the definition of $S$ in Equation (4.47) provides a connection between the domain of convergence for the majorizing IMPPE2 of Theorem 4.4.1(2) and a scalar counterpart for the IMPPE2 of $F(x, y)$ as seen in the following theorem:

Theorem 4.4.3. Given the matrix function $F(x, y)$

$$
F(x, y)=I+\sum_{\substack{p=1 \\ m+n=p}}^{\infty} A_{m, n} x^{m} y^{n}=\operatorname{LTR} \prod_{\substack{q=1 \\ m+n=q}}^{\infty}\left(I-H_{m, n} x^{m} y^{n}\right)^{-1},
$$

with $A_{m, n}, G_{m, n} \in M_{d}(\mathbb{C})$, define the following auxiliary matrices functions with coeffcients over $M_{d}(\mathbb{R})$ :

$$
C(x, y)=I-\sum_{\substack{p=1 \\ m+n=p}}^{\infty}\left|A_{m, n}\right| x^{m} y^{n}=L T R \prod_{\substack{q=1 \\ m+n=q}}^{\infty}\left(I+S_{m, n} x^{m} y^{n}\right)^{-1}
$$




$$
M(x, y)=I-\sum_{\substack{p=1 \\ m+n=p}}^{\infty} M_{m, n} x^{m} y^{n}=\operatorname{LTR} \prod_{\substack{q=1 \\ m+n=q}}^{\infty}\left(I+R_{m, n} x^{m} y^{n}\right)^{-1} .
$$

Let $\|\cdot\|$ be a suitable matrix norm and assume that $\left|A_{m, n}\right| \leq M_{m, n}$ for all $(m, n) \in \mathbb{N}_{0} \times$ $\mathbb{N}_{0} \backslash\{(0,0)\}$. If $\sum_{\substack{p=1 \\ m+n=p}}^{\infty}|| R_{m, n}||\left|x^{m} y^{n}\right|$ converges absolutely in a domain $\mathbb{D}$, then the scalar function $\prod_{\substack{q=1 \\ m+n=q}}^{\infty}\left(1-\left\|H_{m, n}\right\| x^{m} y^{n}\right)^{-1}$ also converges absolutely within $\mathbb{D}$.

Proof. Since $\left|A_{m, n}\right| \leq M_{m, n}$ for all $(m, n) \in \mathbb{N}_{0} \times \mathbb{N}_{0} \backslash\{(0,0)\}$, Theorem 4.4.1(1) implies that

$$
O \leq\left|H_{m, n}\right| \leq R_{m, n} .
$$

Since the norm is suitable, the monotonicity of the matrix inequalities of (4.48) is preserved as the following string of scalar inequalities

$$
0 \leq\left\|H_{m, n}\right\| \leq\left\|R_{m, n}\right\|
$$

and the result follows.

\subsection{Matrix IMPPE2's induced by scalar functions}

In the next theorem we study the expansion of the matrix function $F(A x, A y)$, where $F(x, y)$ is a scalar analytic function.

Theorem 4.5.1. Let $\left(a_{m, n}\right) \in \mathbb{C}$, where $(m, n) \in \mathbb{N}_{0} \times \mathbb{N}_{0} \backslash\{(0,0)\}$. Let

$$
F(x, y)=1+\sum_{\substack{p=1 \\ m+n=p}}^{\infty} a_{m, n} x^{m} y^{n}=\prod_{\substack{q=1 \\ m+n=q}}^{\infty}\left(1-h_{m, n} x^{m} y^{n}\right)^{-1},
$$

where $h_{m, n} \in \mathbb{C},(m, n) \in \mathbb{N}_{0} \times \mathbb{N}_{0} \backslash\{(0,0)\}$ are scalar coefficients.

Let $A \in M_{d}(\mathbb{C})$. Consider the power series $F(A x, A y)$ together with its IMPPE2 expansion

$$
F(A x, A y)=I+\sum_{\substack{p=1 \\ m+n=p}}^{\infty} a_{m, n} A^{m+n} x^{m} y^{n}=L T R \prod_{\substack{q=1 \\ m+n=q}}^{\infty}\left(I-h_{m, n} A^{m+n} x^{m} y^{n}\right)^{-1} .
$$

Let $\left(\lambda_{i}\right)_{i=1}^{p}$ be the distinct eigenvalues of $A$. For $1 \leq i \leq p$, let $n_{i}$ be the algebraic multiplicity of $\lambda_{i}$, let $n_{i}^{g}$ be the geometric multiplicity of $\lambda_{i}$, and let $m_{i}$ be multiplicity of $\lambda_{i}$ as a linear factor within the minimal polynomial $m(\lambda)$. In other words,

$$
\operatorname{det}(\lambda I-A)=\prod_{i=1}^{p}\left(\lambda-\lambda_{i}\right)^{n_{i}} \quad m(\lambda)=\prod_{i=1}^{p}\left(\lambda-\lambda_{i}\right)^{m_{i}}
$$


Define

$$
\rho(A):=\max _{i}\left|\lambda_{i}\right| \quad i=1,2, \ldots, p, \quad s:=\sup _{m+n \geq 1}\left|a_{m, n}\right|^{\frac{1}{m+n}} .
$$

(1.) Consider the scalar power series $M(x, y)$ together with its IPPE2 expansion

$$
M(x, y)=1-\sum_{\substack{p=1 \\ m+n=p}}^{\infty} s^{m+n} x^{m} y^{n}=\prod_{\substack{q=1 \\ m+n=q}}^{\infty}\left(1+r_{m, n} x^{m} y^{n}\right)^{-1},
$$

where $\left(r_{m, n}\right)$ is a sequence of non-negative real numbers determined by certain polynomials in s; see Theorem 2.4.1. Let $\|\cdot\|$ be a suitable norm and

$$
\begin{aligned}
M(\rho(A) K x, \rho(A) K y) & =I-\sum_{\substack{p=1 \\
m+n=p}}^{\infty}[s \rho(A) K]^{m+n} x^{m} y^{n} \\
& =\prod_{\substack{q=1 \\
m+n=q}}^{\infty}\left(I+r_{m, n}[\rho(A) K]^{m+n} x^{m} y^{n}\right)^{-1} .
\end{aligned}
$$

The IMPPE2 of Equation (4.53) converges absolutely in the sense of inverse convergence over the domain

$$
\mathbb{D}_{1}=\left\{(x, y) \in \mathbb{C}^{2}: 2 s \rho(A)(|x|+|y|)+2[s \rho(A)]^{2}|x||y|<1\right\} .
$$

Furthermore, the matrix $F(A x, A y)$ is invertible in $\mathbb{D}_{1}$ and its inverse $F(x, y)^{-1}=$ $R T L \prod_{\substack{q=1 \\ m+n=q}}^{\infty}\left(I-h_{m, n} A^{m+n} x^{m} y^{n}\right)$ is absolutely convergent $\mathbb{D}_{1}$.

(2.) Define $\hat{M}(\rho(A) K x, \rho(A) K y)$, a majorant of $M(\rho(A) K x, \rho(A) K y)$ as follows:

$$
\begin{aligned}
\hat{M}(\rho(A) K x, \rho(A) K y) & =I-\sum_{q=1}^{\infty}(s \rho(A) K x+s \rho(A) K y)^{q} \\
& =L T R \prod_{\substack{q=1 \\
m+n=q}}^{\infty}\left(I+\hat{r}_{m, n}[s \rho(A) K]^{m+n} x^{m} y^{n}\right)^{-1} .
\end{aligned}
$$

The IMPPE2 LTR $\prod_{\substack{q=1 \\ m+n=q}}^{\infty}\left(I-h_{m, n} A^{m+n} x^{m} y^{n}\right)^{-1}$ possess an absolutely convergent infinite product representation in the sense of Definition 4.4 .4 in the polydiscs

$$
\mathbb{D}_{2}=\left\{(x, y) \in \mathbb{C}^{2}:|x|<\frac{1}{4 s \rho(A)\|K\|} \text { and }|y|<\frac{1}{4 s \rho(A)\|K\|}\right\},
$$


with $K \in M_{d}(\mathbb{C})$,

$$
K=K_{1} \bigoplus K_{2} \bigoplus \cdots \bigoplus K_{p}
$$

where for $1 \leq i \leq p$,

$$
K_{i}=K_{i_{1}} \bigoplus K_{i_{2}} \bigoplus \cdots \bigoplus K_{i_{i}^{g}}
$$

with $K_{i_{j}}$ an $m_{i_{j}} \times m_{i_{j}}$ matrix as described below, $m_{i_{j}} \leq m_{i_{1}}=m_{i}$, and $\sum_{j=1}^{n_{i}^{g}} m_{i_{j}}=n_{i}$. Note if $m_{i_{j}}=1, K_{i_{j}}=[1]$, otherwise $K_{i_{j}}=I_{m_{i_{j}}}+N_{m_{i_{j}}}$ where $I_{m_{i_{j}}}$ is the $m_{i_{j}} \times m_{i_{j}}$ identity matrix, while $N_{m_{i j}}$ is the $m_{i_{j}} \times m_{i_{j}}$ the lower triangular nilpotent matrix associated with the Jordan block decomposition, i.e.

$$
K_{m_{i_{j}}}=I_{m_{i_{j}}}+N_{m_{i_{j}}}=\left(\begin{array}{cccccc}
1 & 0 & \ldots & \ldots & 0 & 0 \\
1 & 1 & 0 & \ldots & 0 & 0 \\
0 & 1 & 1 & \ddots & 0 & \vdots \\
\vdots & 0 & \ddots & \ddots & 0 & 0 \\
\vdots & \vdots & \ddots & \ddots & \ddots & 0 \\
0 & \ldots & 0 & 0 & 1 & 1
\end{array}\right) .
$$

Proof. It is known that given a matrix $A \in M_{d}(\mathbb{C})$ and an arbitrary small fixed $\varepsilon \leq \rho(A)$, there exists an invertible matrix $T$ such that

$$
J=T^{-1} A T,
$$

where

$$
J=J_{1} \bigoplus J_{2} \bigoplus \cdots \bigoplus J_{p}
$$

such that for $1 \leq i \leq p$,

$$
J_{i}=J_{i_{1}} \bigoplus J_{i_{2}} \bigoplus \cdots \bigoplus J_{i_{n_{i}^{g}}}
$$

with $J_{i_{j}}$ the $m_{i_{j}} \times m_{i_{j}}$ matrix as described below, $m_{i_{j}} \leq m_{i_{1}}=m_{i}$, and $\sum_{j=1}^{n_{i}^{g}} m_{i_{j}}=n_{i}$. If $m_{i_{j}}=1, J_{i_{j}}=\left[\lambda_{i}\right]$, otherwise $J_{i_{j}}=\lambda_{i} I_{m_{i_{j}}}+\varepsilon N_{m_{i_{j}}}$ where $I_{m_{i_{j}}}$ is the $m_{i_{j}} \times m_{i_{j}}$ identity matrix, while $N_{m_{i_{j}}}$ is the $m_{i_{j}} \times m_{i_{j}}$ the lower triangular nilpotent matrix associated with the Jordan block decomposition, i.e.

$$
J_{i_{j}}=\left(\begin{array}{cccccc}
\lambda_{i} & 0 & \ldots & \ldots & 0 & 0 \\
\varepsilon & \lambda_{i} & 0 & \ldots & 0 & 0 \\
0 & \ddots & \ddots & \ddots & 0 & \vdots \\
\vdots & 0 & \ddots & \ddots & 0 & 0 \\
\vdots & \vdots & \ddots & \ddots & \ddots & 0 \\
0 & \ldots & 0 & 0 & \varepsilon & \lambda_{i}
\end{array}\right)=\lambda_{i} I_{m_{i_{j}}}+\varepsilon N_{m_{i_{j}}}
$$


By using the similarity transformation of Equation (4.59), we have

$$
\begin{aligned}
& F(J x, J y)=I+\sum_{\substack{p=1 \\
m+n=p}}^{\infty} a_{m, n} J^{m+n} x^{m} y^{n}=I+\sum_{\substack{p=1 \\
m+n=p}}^{\infty} a_{m, n}\left(T^{-1} A T\right)^{m+n} x^{m} y^{n} \\
& =T^{-1}\left[I+\sum_{\substack{p=1 \\
m+n=p}}^{\infty} a_{m, n} A^{m+n} x^{m} y^{n}\right] T=\operatorname{LTR} \prod_{\substack{q=1 \\
m+n=q}}^{\infty}\left(I-h_{m, n} J^{m+n} x^{m} y^{n}\right)^{-1} \\
& =\operatorname{LTR} \prod_{\substack{q=1 \\
m+n=q}}^{\infty}\left(I-h_{m, n}\left(T^{-1} A T\right)^{m+n} x^{m} y^{n}\right)^{-1} \\
& =\operatorname{LTR} \prod_{\substack{q=1 \\
m+n=q}}^{\infty}\left(I+\sum_{\alpha=1}^{\infty}\left[h_{m, n}\left(T^{-1} A T\right)^{m+n} x^{m} y^{n}\right]^{\alpha}\right) \\
& =\operatorname{LTR} \prod_{\substack{q=1 \\
m+n=q}}^{\infty} T^{-1}\left(I+\sum_{\alpha=1}^{\infty}\left[h_{m, n} A^{m+n} x^{m} y^{n}\right]^{\alpha}\right) T \\
& =\operatorname{LTR} \prod_{\substack{q=1 \\
m+n=q}}^{\infty} T^{-1}\left(I-h_{m, n} A^{m+n} x^{m} y^{n}\right)^{-1} T \\
& =T^{-1}\left[\operatorname{LTR} \prod_{\substack{q=1 \\
m+n=q}}^{\infty}\left(I-h_{m, n} A^{m+n} x^{m} y^{n}\right)^{-1}\right] T=T^{-1}[F(A x, A y)] T .
\end{aligned}
$$

Consequently, the power series and the IMPPE2 in Equation (4.50) converge absolutely if and only if the power series and its associated IMPPE2 in

$$
F(J x, J y)=I+\sum_{\substack{p=1 \\ m+n=p}}^{\infty} a_{m, n} J^{m+n} x^{m} y^{n}=L T R \prod_{\substack{q=1 \\ m+n=q}}^{\infty}\left(I-h_{m, n} J^{m+n} x^{m} y^{n}\right)^{-1}
$$

converge respectively.

To determine the domain of inverse convergence, we consider two cases. First assume that $\rho(A)=0$. As a result, $A$ is a nilpotent matrix and for some integer $P$ the equation $A^{(P+1)}=O$ holds. For $F(x, y)=1+\sum_{\substack{p=1 \\ m+n=p}}^{\infty} a_{m, n} x^{m} y^{n}, A$ nilpotent implies that

$$
F(A x, A y)=I+\sum_{\substack{p=1 \\ m+n=p}}^{P} a_{m, n} A^{m+n} x^{m} y^{n}=\operatorname{LTR} \prod_{\substack{q=1 \\ m+n=q}}^{\infty}\left(I-h_{m, n} A^{m+n} x^{m} y^{n}\right)^{-1},
$$

is a finite polynomial in $\mathbb{C}^{2}$. 
Now assume that $\rho(A)>0$ and $\varepsilon<\rho(A)$. Thus

$$
|J| \leq \rho(A) K,
$$

where $K$ is defined in Equations (4.56) and (4.58). By using (4.52) and (4.62) and requiring that $|x|<\frac{1}{2 s \rho(A)|| K||}$ and that $|y|<\frac{1}{2 s \rho(A)|| K||}$, we find that

$$
\begin{aligned}
& M(\rho(A) K x, \rho(A) K y)=I-\sum_{\substack{p=1 \\
m+n=p}}^{\infty}[s \rho(A) K]^{m+n} x^{m} y^{n} \\
& =I-\left[\sum_{m=0}^{\infty}(s \rho(A) K x)^{m} \sum_{n=0}^{\infty}(s \rho(A) K y)^{n}-I\right] \\
& =I-\left[(I-s \rho(A) K x)^{-1}(I-s \rho(A) K y)^{-1}-I\right] \\
& =I-(I-s \rho(A) K x)^{-1}(I-s \rho(A) K y)^{-1}[I-(I-s \rho(A) K x)(I-s \rho(A) K y)] \\
& =(I-s \rho(A) K x)^{-1}(I-s \rho(A) K y)^{-1}[2(I-s \rho(A) K x)(I-s \rho(A) K y)-I] \\
& =(I-s \rho(A) K x)^{-1}(I-s \rho(A) K y)^{-1}\left[I-2[s \rho(A) K](x+y)+2[s \rho(A) K]^{2} x y\right] \\
& =L T R \prod_{q=1}^{\infty}\left(I+r_{m, n}[\rho(A) K]^{m+n} x^{m} y^{n}\right)^{-1}, \\
& \quad \\
& m+n=q
\end{aligned}
$$

To obtain the domain of inverse convergence as provided by (4.54), since $K$ is a lower triangular matrix with ones in all the diagonal entries, all of the eigenvalues of $K$ are 1 . Equation (4.63) shows that eigenvalues of $M(\rho(A) K x, \rho(A) K y)$ are $\frac{1-2 s \rho(A)(x+y)+2[s \rho(A)]^{2} x y}{(1-s \rho(A) x)(1-s \rho(A) y)}$. As a result, the eigenvalues of $M(\rho(A) K x, \rho(A) K y)^{-1}$ are $\frac{(1-s \rho(A) x)(1-s \rho(A) y)}{1-2 s \rho(A)(x+y)+2[s \rho(A)]^{2} x y}$. Note that $\frac{(1-s \rho(A) x)(1-s \rho(A) y)}{1-2 s \rho(A)(x+y)+2[s \rho(A)]^{2} x y}=0$ or $\infty$ if and only if $s \rho(A) x=$ 1 , $s \rho(A) y=1$, or $1-2 s \rho(A)(x+y)+2[s \rho(A)]^{2} x y=0$. Thus the nearest singularities of $M(\rho(A) K x, \rho(A) K y)^{-1}$ to $(x, y)=(0,0)$ are the the points $(x, y)$ satisfy the equation $1-2 s \rho(A)(x+y)+2[s \rho(A)]^{2} x y=0$, and the conclusion of (4.54) follows. Since $O \leq$ $\left|h_{m, n} J^{m+n}\right| \leq r_{m, n}[\rho(A) K]^{m+n}$, then $F(J x, J y)^{-1}$ and $F(A x, A y)^{-1}$ are also well defined and absolutely convergent over domain $\mathbb{D}_{1}$.

In order to investigate the convergence of $L T R \prod_{\substack{q=1 \\ m+n=q}}^{\infty}\left(I-h_{m, n} J^{m+n} x^{m} y^{n}\right)^{-1}$ in the sense of Definition 4.4.4, we define a majorant of $M(\rho(A) K x, \rho(A) K y)$, namely

$$
\hat{M}(\rho(A) K x, \rho(A) K y)=I-\sum_{q=1}^{\infty}(s \rho(A) K x+s \rho(A) K y)^{q}
$$




$$
\begin{aligned}
& =I-\sum_{q=1}^{\infty} \sum_{\substack{k=0 \\
k}}^{q}\left(\begin{array}{l}
q \\
k
\end{array}\right)[s \rho(A) K x]^{q-k}[s \rho(A) K y]^{k} \quad \text { Set } m=q-k \text { and } n=k \\
& =I-\sum_{\substack{p=1 \\
m+n=p}}^{\infty}\left(\begin{array}{c}
m+n \\
n
\end{array}\right)[s \rho(A) K]^{m+n} x^{m} y^{n}=I-\sum_{\substack{p=1 \\
m+n=p}}^{\infty} \hat{M}_{m, n} x^{m} y^{n} \\
& =\operatorname{LTR} \prod_{\substack{q=1 \\
m+n=q}}^{\infty}\left(I+\hat{r}_{m, n}[s \rho(A) K]^{m+n} x^{m} y^{n}\right)^{-1},
\end{aligned}
$$

where $\hat{M}_{m, n}=\left(\begin{array}{c}m+n \\ n\end{array}\right)[s \rho(A) K]^{m+n}$.

Since $[s \rho(A) K]^{m+n} \leq \hat{M}_{m, n}$, the structure property applied to (4.53) and (4.61) shows that

$$
h_{m, n} J^{m+n} \leq r_{m, n}[\rho(A) K]^{m+n} \leq \hat{r}_{m, n}[\rho(A) K]^{m+n} .
$$

Next we invert $\hat{M}(\rho(A) K x, \rho(A) K y)$ since this inversion will provide an upper bound for $\hat{r}_{m, n}[\rho(A) K]^{m+n}$. In particular, we have

$$
\begin{aligned}
{[\hat{M}(\rho(A) K x, \rho(A) K y)]^{-1} } & =I+\sum_{\substack{p=1 \\
m+n=p}}^{\infty} \tilde{M}_{m, n} x^{m} y^{n} \\
& =R T L \prod_{\substack{q=1 \\
m+n=q}}^{\infty}\left(I+\hat{r}_{m, n}[s \rho(A) K]^{m+n} x^{m} y^{n}\right)
\end{aligned}
$$

Since $\hat{r}_{m, n}[s \rho(A) K]^{m+n}>O$ for each $(m, n) \in \mathbb{N}_{0} \times \mathbb{N}_{0} \backslash\{(0,0)\}$, both the MPPE2 $R T L \prod_{\substack{q=1 \\ m+n=q}}^{\infty}(I+$ $\left.\hat{r}_{m, n}[s \rho(A) K]^{m+n} x^{m} y^{n}\right)$ and its power series $I+\sum_{\substack{p=1 \\ m+n=p}}^{\infty} \tilde{M}_{m, n} x^{m} y^{n}$ have the same domain of convergence. Moreover, coefficients comparison shows that

$$
O \leq \hat{r}_{m, n}[s \rho(A) K]^{m+n} \leq \tilde{M}_{m, n} .
$$

Combing Equations (4.65) and (4.67) together implies that

$$
\begin{aligned}
\left\|\sum_{\substack{p=1 \\
m+n=p}}^{\infty} \sum_{k=1}^{\infty}\left(h_{m, n} J^{m+n} x^{m} y^{n}\right)^{k}\right\| & \leq \sum_{\substack{p=1 \\
m+n=p}}^{\infty} \sum_{k=1}^{\infty}\left\|h_{m, n} J^{m+n}\right\|^{k}|x|^{m k}|y|^{n k} \\
& \leq \sum_{\substack{p=1 \\
m+n=p}}^{\infty} \sum_{k=1}^{\infty}\left\|\hat{r}_{m, n}[\rho(A) K]^{m+n}\right\| \|^{k}|x|^{m k}|y|^{n k}
\end{aligned}
$$




$$
\leq \sum_{\substack{p=1 \\ m+n=p}}^{\infty} \sum_{k=1}^{\infty}\left\|\tilde{M}_{m, n}\right\|^{k}|x|^{m k}|y|^{n k} .
$$

Equation (4.68) shows that we need to determine an upper bound for $\tilde{M}_{m, n}$. To find the desired upper bound, recall from (4.64) that

$$
\begin{aligned}
& \hat{M}(\rho(A) K x, \rho(A) K y)=I-\sum_{q=1}^{\infty}(s \rho(A) K x+s \rho(A) K y)^{q} \\
& =I-(s \rho(A) K x+s \rho(A) K y)[I-(s \rho(A) K x+s \rho(A) K y)]^{-1} .
\end{aligned}
$$

To make the calculations easier set $B=s \rho(A) K(x+y)$ and assume that $\|B\|<\rho<1$ to obtain

$$
\hat{M}(\rho(A) K x, \rho(A) K y)=I-B[I-B]^{-1} .
$$

Since $\|B\|<1$, we further require that $\|B\|\|I-B\|^{-1} \leq\|I-B\|^{-1}<1$.

Then we have

$$
\begin{aligned}
{[\hat{M}(\rho(A) K x, \rho(A) K y)]^{-1} } & =\left[I-B(I-B)^{-1}\right]^{-1}=I+\sum_{n=1}^{\infty}\left[B(I-B)^{-1}\right]^{n} \\
& =I+B(I-B)^{-1}\left[I-B(I-B)^{-1}\right]^{-1} \\
& =I+B(I-B)^{-1}\left[(I-B)^{-1}((I-B)-B)\right]^{-1} \\
& =I+B(I-2 B)^{-1} \\
& =I+B \sum_{\alpha=0}^{\infty}(2 B)^{\alpha}=I+\sum_{\alpha=1}^{\infty} 2^{\alpha-1} B^{\alpha},
\end{aligned}
$$

where for the second to last equality we assumed $\|B\|<1 / 2$. In Equation (4.71) we set $B=s \rho(A) K(x+y)$ to obtain

$$
\begin{aligned}
& {[\hat{M}(\rho(A) K x, \rho(A) K y)]^{-1}=I+\sum_{\alpha=1}^{\infty} 2^{\alpha-1}[s \rho(A) K]^{\alpha}(x+y)^{\alpha}} \\
& =I+\sum_{\alpha=1}^{\infty} 2^{\alpha-1}[s \rho(A) K]^{\alpha} \sum_{p=0}^{\alpha}\left(\begin{array}{c}
\alpha \\
p
\end{array}\right) x^{p} y^{\alpha-p} \\
& =I+\sum_{\alpha=1}^{\infty} 2^{\alpha-1}[s \rho(A) K]^{\alpha}\left[y^{\alpha}+\sum_{p=1}^{\alpha}\left(\begin{array}{c}
\alpha \\
p
\end{array}\right) x^{p} y^{\alpha-p}\right] \\
& =I+\sum_{\alpha=1}^{\infty} 2^{\alpha-1}[s \rho(A) K]^{\alpha} y^{\alpha}+\sum_{\alpha=1}^{\infty} 2^{\alpha-1}[s \rho(A) K]^{\alpha} \sum_{p=1}^{\alpha}\left(\begin{array}{c}
\alpha \\
p
\end{array}\right) x^{p} y^{\alpha-p}
\end{aligned}
$$




$$
\begin{aligned}
& =I+\sum_{\alpha=1}^{\infty} 2^{\alpha-1}[s \rho(A) K]^{\alpha} y^{\alpha}+\sum_{p=1}^{\infty} \sum_{\alpha=p}^{\infty} 2^{\alpha-1}\left(\begin{array}{c}
\alpha \\
p
\end{array}\right)[s \rho(A) K]^{\alpha} x^{p} y^{\alpha-p} \\
& =I+\sum_{\substack{p=1 \\
m+n=1}}^{\infty} 2^{m+n-1}[s \rho(A) K]^{m+n}\left(\begin{array}{c}
m+n \\
n
\end{array}\right) x^{m} y^{n}, \quad p=m, \alpha-p=n \\
& =I+\sum_{\substack{p=1 \\
m+n=1}}^{\infty} \tilde{M}_{m, n} x^{m} y^{n},
\end{aligned}
$$

where $\tilde{M}_{m, n}=2^{m+n-1}[s \rho(A) K]^{m+n}\left(\begin{array}{c}m+n \\ n\end{array}\right)$. So we have

$$
\tilde{M}_{m, n}=2^{m+n-1}[s \rho(A) K]^{m+n}\left(\begin{array}{c}
m+n \\
n
\end{array}\right) \leq 2^{2(m+n)-1}[s \rho(A) K]^{m+n}
$$

where for the last inequality we made use of the fact that the sum of all the entries in the $m+n$-th row of Pascal's triangle is equal to $2^{m+n}$.

Plugging the desired upper bound of (4.73) into (4.68), we find that

$$
\begin{aligned}
\| & \sum_{\substack{p=1 \\
m+n=p}}^{\infty} \sum_{k=1}^{\infty}\left(h_{m, n} J^{m+n} x^{m} y^{n}\right)^{k}\left\|\leq \sum_{\substack{p=1 \\
m+n=p}}^{\infty} \sum_{k=1}^{\infty}\right\| \tilde{M}_{m, n} \|^{k}|x|^{m k}|y|^{n k} \\
\leq & \sum_{\substack{p=1 \\
m+n=p}}^{\infty} \sum_{k=1}^{\infty}\left\|2^{2(m+n)-1}[s \rho(A) K]^{m+n}\right\|^{k}|x|^{m k}|y|^{n k} \\
\leq & \frac{1}{2} \sum_{\substack{p=1 \\
m+n=p}}^{\infty} \sum_{k=1}^{\infty}\left\|[4 s \rho(A) K]^{m+n} x^{m} y^{n}\right\|^{k} \\
= & \frac{1}{2} \sum_{\substack{p=1 \\
m+n=p}}^{\infty}\left[\|4 s \rho(A) K\|^{m+n}|x|^{m}|y|^{n}\right]\left[1-[\|4 s \rho(A) K\||x|]^{m}[\|4 s \rho(A) K\||y|]^{n}\right]^{-1} .
\end{aligned}
$$

where in the penultimate line we assumed $\left\|[4 s \rho(A) K]^{m+n} x^{m} y^{n}\right\|<1$. This assumption provides a further refinement on the polydiscs used in the computation of (4.71) where we assumed that $s \rho(A)\|K\||x|<\rho_{1}<\rho<1$ and $s \rho(A)\|K\||y|<\rho_{2}<\rho<1$. Now we have to shrink these polydiscs and require that $4 \sin (A)\|K\||x|<\rho_{1}<\rho<1$ and that $4 s \rho(A)\|K\||y|<\rho_{2}<\rho<1$. When working over these refined polydiscs, the previous inequity becomes

$$
\left\|\sum_{\substack{p=1 \\ m+n=p}}^{\infty} \sum_{k=1}^{\infty}\left(h_{m, n} J^{m+n} x^{m} y^{n}\right)^{k}\right\| \leq \frac{1}{2\left(1-\rho^{2}\right)} \sum_{\substack{p=1 \\ m+n=p}}^{\infty}[4 s \rho(A)\|K\||x|]^{m}[4 s \rho(A)\|K\||y|]^{n}
$$




$$
\begin{aligned}
& \leq \frac{1}{2(1-\rho)} \sum_{\substack{p=1 \\
m+n=p}}^{\infty} \rho^{m+n}=\frac{1}{2(1-\rho)}\left[\sum_{m=0}^{\infty} \rho^{m} \sum_{n=0}^{\infty} \rho^{n}-1\right] \\
& =\frac{2 \rho-\rho^{2}}{2(1-\rho)^{3}} .
\end{aligned}
$$

In conclusion, by using a majorant of a majorant and clever use of inversion, we finally showed

$$
\begin{aligned}
\left\|\sum_{\substack{p=1 \\
m+n=p}}^{\infty} \sum_{k=1}^{\infty}\left(h_{m, n} J^{m+n} x^{m} y^{n}\right)^{k}\right\| & \leq \sum_{\substack{p=1 \\
m+n=p}}^{\infty} \sum_{k=1}^{\infty}\left\|\tilde{M}_{m, n}\right\|^{k}|x|^{m k}|y|^{n k} \\
& =\frac{2 \rho-\rho^{2}}{2\left(1-\rho^{2}\right)^{3}},
\end{aligned}
$$

whenever $4 \operatorname{s} \rho(A)\|K\||x|<\rho<1$ and $4 s \rho(A)\|K\||y|<\rho<1$.

Therefore, an estimate for domain of absolute convergence of $\operatorname{LTR} \prod_{\substack{q=1 \\ m+n=q}}^{\infty}\left(I+\hat{r}_{m, n}[s \rho(A) K]^{m+n} x^{m} y^{n}\right)^{-1}$ is

$$
\mathbb{D}_{2}=\left\{(x, y) \in \mathbb{C}^{2}:|x|<\frac{1}{4 s \rho(A)\|K\|} \text { and }|y|<\frac{1}{4 s \rho(A)\|K\|}\right\} .
$$

Since $\left|h_{m, n} J^{m+n}\right| \leq r_{m, n}[\rho(A) K]^{m+n} \leq \hat{r}_{m, n}[\rho(A) K]^{m+n}$, Definition 4.4.4 implies that $\mathbb{D}_{2}$ is also an estimate for the domain of absolute convergence of

$$
\operatorname{LTR} \prod_{\substack{q=1 \\ m+n=q}}^{\infty}\left(I-h_{m, n} J^{m+n} x^{m} y^{n}\right)^{-1} \text { and of } \operatorname{LTR} \prod_{\substack{q=1 \\ m+n=q}}^{\infty}\left(I-h_{m, n} A^{m+n} x^{m} y^{n}\right)^{-1} .
$$

In the next theorem we study the expansion of the matrix function $F(B x, C y)$, where $F(x, y)$ is a scalar analytic function and $B, C \in M_{d}(\mathbb{C})$ are both diagonalizable with $B C=$ $C B$.

Theorem 4.5.2. Let $\left(a_{m, n}\right)$ be a sequence of complex numbers, where $(m, n) \in \mathbb{N}_{0} \times \mathbb{N}_{0} \backslash$ $\{(0,0)\}$. Let

$$
F(x, y)=1+\sum_{\substack{p=1 \\ m+n=p}}^{\infty} a_{m, n} x^{m} y^{n}=\prod_{\substack{q=1 \\ m+n=q}}^{\infty}\left(1-h_{m, n} x^{m} y^{n}\right)^{-1},
$$

where $h_{m, n} \in \mathbb{C},(m, n) \in \mathbb{N}_{0} \times \mathbb{N}_{0} \backslash\{(0,0)\}$ are scalar coefficients.

Let $B$ and $C$ be $d \times d$ commutative diagonalizable matrices. Consider the power series $F(B x, C y)$ together with its IMPPE2 expansion

$$
F(B x, C y)=I+\sum_{\substack{p=1 \\ m+n=p}}^{\infty} a_{m, n} B^{m} C^{n} x^{m} y^{n}=\operatorname{LTR} \prod_{\substack{q=1 \\ m+n=q}}^{\infty}\left(I-h_{m, n} B^{m} C^{n} x^{m} y^{n}\right)^{-1} .
$$


Let $\left(\lambda_{i}\right)_{i=1}^{p},\left(\lambda_{i}^{\prime}\right)_{i=1}^{p}$ be the distinct eigenvalues of $B$ and $C$ respectively. Define

$$
\begin{aligned}
\rho(B) & :=\max _{i}\left|\lambda_{i}\right| \quad i=1,2, \ldots, p \\
\rho(C) & :=\max _{i}\left|\lambda_{i}^{\prime}\right| \quad i=1,2, \ldots, p \\
s & :=\sup _{m+n \geq 1}\left|a_{m, n}\right|^{\frac{1}{m+n}} .
\end{aligned}
$$

(1.) Consider the scalar power series $M(x, y)$ together with its IPPE2 expansion

$$
M(x, y)=1-\sum_{\substack{p=1 \\ m+n=p}}^{\infty} s^{m+n} x^{m} y^{n}=\prod_{\substack{q=1 \\ m+n=q}}^{\infty}\left(1+r_{m, n} x^{m} y^{n}\right)^{-1},
$$

where $\left(r_{m, n}\right)$ is a sequence of non-negative real numbers determined by certain polynomials in s; see Theorem 2.4.1. Let $\|\cdot\|$ be a suitable norm and

$$
\begin{aligned}
M(\rho(B) I x, \rho(C) I y) & =I-\sum_{\substack{p=1 \\
m+n=p}}^{\infty}[s \rho(B) I]^{m}[s \rho(C) I]^{n} x^{m} y^{n} \\
& =\prod_{\substack{q=1 \\
m+n=q}}^{\infty}\left(1+r_{m, n}[\rho(B) I]^{m}[\rho(C) I]^{n} x^{m} y^{n}\right)^{-1}
\end{aligned}
$$

The IMPPE2 of Equation (4.78) converges absolutely in the sense of inverse convergence over the domain

$$
\mathbb{D}_{1}=\left\{(x, y) \in \mathbb{C}^{2}: 2 s[\rho(B)|x|+\rho(C)|y|]+2[s \rho(B) \rho(C)]^{2}|x||y|<1\right\} .
$$

Furthermore, the matrix $F(B x, C y)$ is invertible in $\mathbb{D}_{1}$ and its inverse $F(B x, C y)^{-1}=$ $R T L \prod_{\substack{q=1 \\ m+n=q}}^{\infty}\left(I-h_{m, n} B^{m} C^{n} x^{m} y^{n}\right)$ is absolutely convergent $\mathbb{D}_{1}$.

(2.) Define $\hat{M}(\rho(B) I x, \rho(C) I y)$, a majorant of $M(\rho(B) I x, \rho(C) I y)$ as follows:

$$
\begin{aligned}
\hat{M}(\rho(B) I x, \rho(C) I y) & =I-\sum_{\substack{q=1 \\
\infty}}^{\infty}(s \rho(B) I x+s \rho(C) I y)^{q} \\
& =L T R \prod_{\substack{q=1 \\
m+n=q}}^{\infty}\left(I+\hat{r}_{m, n}[s \rho(B) I]^{m}[s \rho(C) I]^{n} x^{m} y^{n}\right)^{-1} .
\end{aligned}
$$

1 
Proof. Let $B$ and $C$ be two commutative diagonalizable matrices. By Proposition 6.2.6 of [40] there is a single invertible matrix $S \in M_{d}(\mathbb{C})$ such that

$$
\begin{aligned}
& D_{1}=S^{-1} B S \\
& D_{2}=S^{-1} C S,
\end{aligned}
$$

where

$$
D_{1}=\left(\begin{array}{cccccc}
\lambda_{1} & 0 & \ldots & \ldots & 0 & 0 \\
0 & \lambda_{2} & 0 & \ldots & 0 & 0 \\
0 & \ddots & \ddots & \ddots & 0 & \vdots \\
\vdots & 0 & \ddots & \ddots & 0 & 0 \\
\vdots & \vdots & \ddots & \ddots & \ddots & 0 \\
0 & \ldots & 0 & 0 & 0 & \lambda_{p}
\end{array}\right), D_{2}=\left(\begin{array}{cccccc}
\lambda_{1}^{\prime} & 0 & \ldots & \ldots & 0 & 0 \\
0 & \lambda_{2}^{\prime} & 0 & \ldots & 0 & 0 \\
0 & \ddots & \ddots & \ddots & 0 & \vdots \\
\vdots & 0 & \ddots & \ddots & 0 & 0 \\
\vdots & \vdots & \ddots & \ddots & \ddots & 0 \\
0 & \ldots & 0 & 0 & 0 & \lambda_{p}^{\prime}
\end{array}\right)
$$

By using the similarity transformations of (4.85) and (4.86), we have

$$
\begin{aligned}
& F\left(D_{1} x, D_{2} y\right)=I+\sum_{\substack{p=1 \\
m+n=p}}^{\infty} a_{m, n} D_{1}^{m} D_{2}^{n} x^{m} y^{n}=I+\sum_{\substack{p=1 \\
m+n=p}}^{\infty} a_{m, n}\left(S^{-1} B S\right)^{m}\left(S^{-1} C S\right)^{n} x^{m} y^{n} \\
& =S^{-1}\left[I+\sum_{\substack{p=1 \\
m+n=p}}^{\infty} a_{m, n} B^{m} C^{n} x^{m} y^{n}\right] S=L T R \prod_{\substack{q=1 \\
m+n=q}}^{\infty}\left(I-h_{m, n} D_{1}^{m} D_{2}^{n} x^{m} y^{n}\right)^{-1} \\
& =\operatorname{LTR} \prod_{\substack{q=1 \\
m+n=q}}^{\infty}\left(I-h_{m, n}\left(S^{-1} B S\right)^{m}\left(S^{-1} C S\right)^{n} x^{m} y^{n}\right)^{-1} \\
& \left.=\operatorname{LTR} \prod_{\substack{q=1 \\
m+n=q}}^{\infty}\left(I+\sum_{\alpha=1}^{\infty}\left[h_{m, n}\left(S^{-1} B S\right)^{m}\left(S^{-1} C S\right)^{n} x^{m} y^{n}\right) x^{m} y^{n}\right]^{\alpha}\right) \\
& =\operatorname{LTR} \prod_{\substack{q=1 \\
m+n=q}}^{\infty} S^{-1}\left(I+\sum_{\alpha=1}^{\infty}\left[h_{m, n} B^{m} C^{n} x^{m} y^{n}\right]^{\alpha}\right) S \\
& =\operatorname{LTR} \prod_{\substack{q=1 \\
m+n=q}}^{\infty} S^{-1}\left(I-h_{m, n} B^{m} C^{n} x^{m} y^{n}\right)^{-1} S \\
& =S^{-1}\left[\operatorname{LTR} \prod_{\substack{q=1 \\
m+n=q}}^{\infty}\left(I-h_{m, n} B^{m} C^{n} x^{m} y^{n}\right)^{-1}\right] S=S^{-1}[F(B x, C y)] S .
\end{aligned}
$$


Consequently, the power series and the IMPPE2 in (4.78) converge absolutely if and only if the power series and its associated IMPPE2 in

$$
F\left(D_{1} x, D_{2} y\right)=I+\sum_{\substack{p=1 \\ m+n=p}}^{\infty} a_{m, n} D_{1}^{m} D_{2}^{n} x^{m} y^{n}=L T R \prod_{\substack{q=1 \\ m+n=q}}^{\infty}\left(I-h_{m, n} D_{1}^{m} D_{2}^{n} x^{m} y^{n}\right)^{-1}
$$

converge absolutely respectively. We begin by discussing the domain of absolute convergence in the inverse sense. Consider two cases: in the first case, assume that $\rho(B)=0$ or $\rho(C)=0$. As a result, $B$ or $C$ is a nilpotent matrix and for some integer $P$ the equation $B^{(P+1)}=O$ or $C^{(P+1)}=O$ holds. For $F(x, y)=1+\sum_{\substack{p=1 \\ m+n=p}}^{\infty} a_{m, n} x^{m} y^{n}, B$ or $C$ nilpotent implies that

$$
F(B x, C y)=I+\sum_{\substack{p=1 \\ m+n=p}}^{P} a_{m, n} B^{m} C^{n} x^{m} y^{n}=\operatorname{LTR} \prod_{\substack{q=1 \\ m+n=q}}^{\infty}\left(I-h_{m, n} B^{m} C^{n} x^{m} y^{n}\right)^{-1},
$$

is a finite polynomial in $\mathbb{C}$.

Now consider the case that $\rho(B), \rho(C)>0$. Then we have

$$
\begin{aligned}
& \left|D_{1}\right| \leq \rho(B) I \\
& \left|D_{2}\right| \leq \rho(C) I,
\end{aligned}
$$

where $I$ is the $d \times d$ the identity matrix. By using (4.82), (4.88) and (4.89), and requiring that $|x|<\frac{1}{s \rho(B)|| I||}$ and that $|y|<\frac{1}{s \rho(C)\|I\|}$ we find that

$$
\begin{aligned}
& M(\rho(B) I x, \rho(C) I y)=I-\sum_{\substack{p=1 \\
m+n=p}}^{\infty}[s \rho(B) I]^{m}[s \rho(C) I]^{n} x^{m} y^{n} \\
= & I-\left[\sum_{m=0}^{\infty}[s \rho(B) I x]^{m} \sum_{n=0}^{\infty}[s \rho(C) I y]^{n}-I\right] \\
= & I-\left[(I-s \rho(B) I x)^{-1}(I-s \rho(C) I y)^{-1}-I\right] \\
= & I-(I-s \rho(B) I x)^{-1}(I-s \rho(C) I y)^{-1}[I-(I-s \rho(B) I x)(I-s \rho(C) I y)] \\
= & (I-s \rho(B) I x)^{-1}(I-s \rho(C) I y)^{-1}[2(I-s \rho(B) I x)(I-s \rho(C) I y)-I] \\
= & (I-s \rho(B) I x)^{-1}(I-s \rho(C) I y)^{-1}[I-2 s[\rho(B) I x+\rho(C) I y]+ \\
& \left.2 s^{2} \rho(B) \rho(C) I x y\right]
\end{aligned}
$$




$$
=L T R \prod_{\substack{q=1 \\ m+n=q}}^{\infty}\left(I+r_{m, n}[\rho(B) I]^{m}[\rho(C) I]^{n} x^{m} y^{n}\right)^{-1} .
$$

To obtain the domain of inverse convergence as provided by (4.84), since $I$ is an identity matrix then all of the eigenvalues of $I$ are 1 . Equation (4.90) shows that eigenvalues of $M(\rho(B) I x, \rho(C) I y)$ are $\frac{1-2 s[(\rho(B) x+\rho(C) y)]+2 s^{2} \rho(B) \rho(C) x y}{(1-s \rho(B) x)(1-s \rho(C) y)}$. As a result, the eigenvalues of $M(\rho(B) I x, \rho(C) I y)^{-1}$ are $\frac{(1-s \rho(B) x)(1-s \rho(C) y)}{1-2 s[(\rho(B) x+\rho(C) y)]+2 s^{2} \rho(B) \rho(C) x y}$. Note that $\frac{(1-s \rho(B) x)(1-s \rho(C) y)}{1-2 s[(\rho(B) x+\rho(C) y)]+2 s^{2} \rho(B) \rho(C) x y}=0$ or $\infty$ if and only if $s \rho(B) x=1$ or $s \rho(C) y=1$, or $1-$ $2 s[(\rho(B) x+\rho(C) y)]+2 s^{2} \rho(B) \rho(C) x y=0$. Thus the nearest singularities of $M(\rho(B) I x, \rho(C) I y)^{-1}$ to $(x, y)=(0,0)$ are the the points $(x, y)$ satisfy the equation $1-$ $2 s[(\rho(B) x+\rho(C) y)]+2 s^{2} \rho(B) \rho(C) x y=0$, and the conclusion of (4.84) follows. Since $O \leq\left|h_{m, n} D_{1}^{m} D_{2}^{n}\right| \leq r_{m, n}[\rho(B) I]^{m}[\rho(C) I]^{n}$, then $F\left(D_{1} x, D_{2} y\right)^{-1}$ and $F(A x, A y)^{-1}$ are also well defined and absolutely convergent over domain $\mathbb{D}_{1}$.

In order to investigate the convergence of $\operatorname{LTR} \prod_{\substack{q=1 \\ m+n=q}}^{\infty}\left(I-h_{m, n} D_{1}^{m} D_{2}^{n} x^{m} y^{n}\right)^{-1}$ in the sense of Definition 4.4.4, we define a majorant of $M(\rho(B) I x, \rho(C) I y)$, namely

$$
\begin{aligned}
& \hat{M}(\rho(B) I x, \rho(C) I y)=I-\sum_{q=1}^{\infty}(s \rho(B) I x+\rho(C) I y)^{q} \\
& =I-\sum_{q=1}^{\infty} \sum_{k=0}^{q}\left(\begin{array}{c}
q \\
k
\end{array}\right)[s \rho(B) I x]^{q-k}[s \rho(C) I y]^{k} \quad \text { Set } m=q-k \text { and } n=k \\
& =I-\sum_{\substack{p=1 \\
m+n=p}}^{\infty}\left(\begin{array}{c}
m+n \\
n
\end{array}\right)[s \rho(B) I]^{m}[s \rho(C) I]^{n} x^{m} y^{n} \\
& =I-\sum_{\substack{p=1 \\
m+n=p}}^{\infty} \hat{M}_{m, n} x^{m} y^{n} \\
& =L T R \prod_{\substack{q=1 \\
m+n=q}}^{\infty}\left(I+\hat{r}_{m, n}[s \rho(B) I]^{m}[s \rho(C) I]^{n} x^{m} y^{n}\right)^{-1},
\end{aligned}
$$

where $\hat{M}_{m, n}=\left(\begin{array}{c}m+n \\ n\end{array}\right)[s \rho(B) I]^{m}[s \rho(C) I]^{n}$.

Since $M_{m, n} \leq \hat{M}_{m, n}$, the structure property applied to (4.78) and (4.87) shows that

$$
h_{m, n} D_{1}^{m} D_{2}^{n} \leq r_{m, n}[\rho(B) I]^{m}[\rho(C) I]^{n} \leq \hat{r}_{m, n}[\rho(B) I]^{m}[\rho(C) I]^{n} .
$$

Next we invert $\hat{M}(\rho(B) I x, \rho(C) I y)$ since this inversion will provide an upper bound for 
$\hat{r}_{m, n}[\rho(B) I]^{m}[\rho(C) I]^{n}$. In particular, we have

$$
\begin{aligned}
{[M(\rho(B) I x, \rho(C) I y)]^{-1} } & =I+\sum_{\substack{p=1 \\
m+n=p}}^{\infty} \tilde{M}_{m, n} x^{m} y^{n} \\
& =R T L \prod_{\substack{q=1 \\
m+n=q}}^{\infty}\left(I+\hat{r}_{m, n}[\rho(B) I]^{m}[\rho(C) I]^{n} x^{m} y^{n}\right) .
\end{aligned}
$$

Since $\hat{r}_{m, n}[\rho(B) I]^{m}[\rho(C) I]^{n}>O$ for each $(m, n) \in \mathbb{N}_{0} \times \mathbb{N}_{0} \backslash\{(0,0)\}$, then both $R T L \prod_{\substack{q=1 \\ m+n=q}}^{\infty}(I+$ $\left.\hat{r}_{m, n}[\rho(B) I]^{m}[\rho(C) I]^{n} x^{m} y^{n}\right), I+\sum_{\substack{p=1 \\ m+n=p}}^{\infty} \tilde{M}_{m, n} x^{m} y^{n}$ have the same domain of convergence. Moreover, coefficients comparison shows that

$$
O \leq \hat{r}_{m, n}[\rho(B) I]^{m}[\rho(C) I]^{n} \leq \tilde{M}_{m, n} .
$$

Combing Equations (4.92) and (4.94) together implies that

$$
\begin{aligned}
& \left\|\sum_{\substack{p=1 \\
m+n=p}}^{\infty} \sum_{k=1}^{\infty}\left(h_{m, n} D_{1}^{m} D_{2}^{n} x^{m} y^{n}\right)^{k}\right\| \leq \sum_{\substack{p=1 \\
m+n=p}}^{\infty} \sum_{k=1}^{\infty}\left\|h_{m, n} D_{1}^{m} D_{2}^{n}\right\|^{k}|x|^{m k}|y|^{n k} \\
& \leq \sum_{\substack{p=1 \\
m+n=p}}^{\infty} \sum_{k=1}^{\infty}\left\|\hat{r}_{m, n}[\rho(B) I]^{m}[\rho(C) I]^{n}\right\|^{k}|x|^{m k}|y|^{n k} \\
& \leq \sum_{\substack{p=1 \\
m+n=p}}^{\infty} \sum_{k=1}^{\infty}\left\|\tilde{M}_{m, n}\right\|^{k}|x|^{m k}|y|^{n k} .
\end{aligned}
$$

Equation (4.95) shows that we need to determine an upper bound for $\tilde{M}_{m, n}$. To find the desired upper bound, recall from (4.91) that

$$
\begin{aligned}
& \hat{M}(\rho(B) I x, \rho(C) I y)=I-\sum_{q=1}^{\infty}(s \rho(B) I x+s \rho(C) I y)^{q} \\
& =I-(s \rho(B) I x+s \rho(C) I y)[I-(s \rho(B) I x+s \rho(C) I y)]^{-1} .
\end{aligned}
$$

To make the calculations easier set $Z=\rho(B) I x+\rho(C) I y$, and assume that $\|Z\|<\rho<1$ to obtain

$$
\hat{M}(Z)=I-Z(I-Z)^{-1} .
$$


Since $\|Z\|<1$, we further require that $\|Z\|\|I-Z\|^{-1} \leq\|I-Z\|^{-1}<1$. Then we have

$$
\begin{aligned}
{[\hat{M}(Z)]^{-1} } & =\left[I-Z(I-Z)^{-1}\right]^{-1} \\
& =I+\sum_{n=1}^{\infty}\left[Z(I-Z)^{-1}\right]^{n}=I+Z[I-Z]^{-1}\left[I-Z[I-Z]^{-1}\right]^{-1} \\
& =I+Z[I-Z]^{-1}\left[[I-Z]^{-1}[(I-Z)-Z]\right]^{-1} \\
& =I+Z(I-2 Z)^{-1}=I+Z \sum_{\alpha=0}^{\infty}(2 Z)^{\alpha} \\
& =I+\sum_{\alpha=1}^{\infty} 2^{\alpha-1} Z^{\alpha},
\end{aligned}
$$

where for the second to last equality we assumed $\|Z\|<\frac{1}{2}$. In Equation (4.98) we set $Z=\rho(B) I x+\rho(C) I y$ to obtain

$$
\begin{aligned}
& {[\hat{M}(\rho(B) I x, \rho(C) I y)]^{-1}=I+\sum_{\alpha=1}^{\infty} 2^{\alpha-1}(\rho(B) I x+\rho(C) I y)^{\alpha}} \\
& =I+\sum_{\alpha=1}^{\infty} 2^{\alpha-1} \sum_{p=0}^{\alpha}\left(\begin{array}{c}
\alpha \\
p
\end{array}\right)[\rho(B) I x]^{p}[\rho(C) I y]^{\alpha-p} \\
& =I+\sum_{\alpha=1}^{\infty} 2^{\alpha-1}\left[[\rho(C) I y]^{\alpha}+\sum_{p=1}^{\alpha}\left(\begin{array}{c}
\alpha \\
p
\end{array}\right)[\rho(B) I x]^{p}[\rho(C) I y]^{\alpha-p}\right] \\
& =I+\sum_{\alpha=1}^{\infty} 2^{\alpha-1}[s \rho(C) I y]^{\alpha}+\sum_{\alpha=1}^{\infty} 2^{\alpha-1} \sum_{p=1}^{\alpha}\left(\begin{array}{c}
\alpha \\
p
\end{array}\right)[\rho(B) I x]^{p}[\rho(C) I y]^{\alpha-p} \\
& =I+\sum_{\alpha=1}^{\infty} 2^{\alpha-1}[s \rho(C) I y]^{\alpha}+\sum_{p=1}^{\infty} \sum_{\alpha=p}^{\infty} 2^{\alpha-1}\left(\begin{array}{c}
\alpha \\
p
\end{array}\right)[\rho(B) I x]^{p}[\rho(C) I y]^{\alpha-p} \\
& =I+\sum_{\substack{p=1 \\
m+n=1}}^{\infty} 2^{m+n-1}[s \rho(B) I]^{m}[s \rho(C) I]^{n}\left(\begin{array}{c}
m+n \\
m
\end{array}\right) x^{m} y^{n}, \quad p=m, \alpha-p=n \\
& =I+\sum_{\substack{p=1 \\
m+n=1}}^{\infty} \tilde{M}_{m, n} x^{m} y^{n},
\end{aligned}
$$

where $\tilde{M}_{m, n}=2^{m+n-1}[s \rho(B) I]^{m}[s \rho(C) I]^{n}\left(\begin{array}{c}m+n \\ m\end{array}\right)$. So, we have

$$
\begin{aligned}
\tilde{M}_{m, n} & =2^{m+n-1}[s \rho(B) I]^{m}[s \rho(C) I]^{n}\left(\begin{array}{c}
m+n \\
n
\end{array}\right) \\
& \leq 2^{2(m+n)-1}[s \rho(B) I]^{m}[s \rho(C) I]^{n},
\end{aligned}
$$


where for the last inequality we made use of the fact that the sum of all the entries in the $m+n$-th row of Pascal's triangle is equal to $2^{m+n}$.

Plugging the desired upper bound of (4.100) into (4.95), we find that

$$
\begin{aligned}
\| & \sum_{\substack{p=1 \\
m+n=p}}^{\infty} \sum_{k=1}^{\infty}\left(h_{m, n} D_{1}^{m} D_{2}^{n} x^{m} y^{n}\right)^{k}\left\|\leq \sum_{\substack{p=1 \\
m+n=p}}^{\infty} \sum_{k=1}^{\infty}\right\| \tilde{M}_{m, n} \|^{k}|x|^{m k}|y|^{n k} \\
\leq & \sum_{\substack{p=1 \\
m+n=p}}^{\infty} \sum_{k=1}^{\infty}\left\|2^{2(m+n)-1}[s \rho(B) I]^{m}[s \rho(C) I]^{n}\right\|^{k}|x|^{m k}|y|^{n k} \\
\leq & \frac{1}{2} \sum_{\substack{p=1 \\
m+n=p}}^{\infty} \sum_{k=1}^{\infty}\left[\|4 s \rho(B) I\|^{m}\|4 s \rho(C) I\|^{n}|x|^{m}|y|^{n}\right]^{k} \\
= & \frac{1}{2} \sum_{\substack{p=1 \\
m+n=p}}^{\infty}\left[\|4 s \rho(B) I\|^{m}\|4 s \rho(C) I\|^{n}|x|^{m}|y|^{n}\right]\left[I-\|4 s \rho(B) I x\|^{m}\|4 s \rho(C) I y\|^{n}\right]^{-1} .
\end{aligned}
$$

where in the penultimate line we assumed $\left\|[4 s \rho(B) I]^{m}[\rho(C) I]^{n} x^{m} y^{n}\right\|<1$. This assumption provides a further refinement on the polydiscs used in the computation of (4.98) where we assumed that $s \rho(B)\|I\||x|<\rho<1$ and $s \rho(C)\|I\||y|<\rho<1$. Now we have to shrink these polydiscs and require that $4 \sin (B)\|I\||x|<\rho_{1}<\rho<1$ and that $4 \sin (C)\|I\||y|<$ $\rho_{2}<\rho<1$. When working over these refined polydiscs, the previous inequity becomes

$$
\begin{aligned}
& \left\|\sum_{\substack{p=1 \\
m+n=p}}^{\infty} \sum_{k=1}^{\infty}\left(h_{m, n} D_{1}^{m} D_{2}^{n} x^{m} y^{n}\right)^{k}\right\| \leq \frac{1}{2\left(1-\rho^{2}\right)} \sum_{\substack{p=1 \\
m+n=p}}^{\infty}[4 s \rho(B)\|I\||x|]^{m}[4 s \rho(C)\|I\||y|]^{n} \\
& \leq \frac{1}{2(1-\rho)} \sum_{\substack{p=1 \\
m+n=p}}^{\infty} \rho^{m+n}=\frac{1}{2(1-\rho)}\left[\sum_{m=0}^{\infty} \rho^{m} \sum_{n=0}^{\infty} \rho^{n}-1\right] \\
& =\frac{2 \rho-\rho^{2}}{2(1-\rho)^{3}}
\end{aligned}
$$

In conclusion, by using a majorant of a majorant and clever use of inversion, we finally showed

$$
\begin{aligned}
\left\|\sum_{\substack{p=1 \\
m+n=p}}^{\infty} \sum_{k=1}^{\infty}\left(h_{m, n} D_{1}^{m} D_{2}^{n} x^{m} y^{n}\right)^{k}\right\| & \leq \sum_{\substack{p=1 \\
m+n=p}}^{\infty} \sum_{k=1}^{\infty}\left\|\tilde{M}_{m, n}\right\|^{k}|x|^{m k}|y|^{n k} \\
& =\frac{2 \rho-\rho^{2}}{2\left(1-\rho^{2}\right)^{3}},
\end{aligned}
$$


whenever $4 \operatorname{s} \rho(B)\|I\||x|<\rho<1$ and $4 s \rho(C)\|I\||y|<\rho<1$.

Therefore, an estimate for domain of absolute convergence of $R T L \prod_{\substack{q=1 \\ m+n=q}}^{\infty}\left(I+\hat{r}_{m, n}[s \rho(B) I]^{m}[s \rho(C) I]^{n} x^{m} y^{n}\right)$ is

$$
\mathbb{D}_{2}=\left\{(x, y) \in \mathbb{C}^{2}:|x|<\frac{1}{4 s \rho(B)\|I\|} \text { and }|y|<\frac{1}{4 s \rho(C)\|I\|}\right\} .
$$

Since $\left|h_{m, n} D_{1}^{m} D_{2}^{n}\right| \leq r_{m, n}[\rho(B) I]^{m}[\rho(C) I]^{n} \leq \hat{r}_{m, n}[\rho(B) I]^{m}[\rho(C) I]^{n}$, Definition 4.4 .4 implies that $\mathbb{D}_{2}$ is also an estimate for the domain of absolute convergence of $\operatorname{LTR} \prod_{\substack{q=1 \\ m+n=q}}^{\infty}\left(I-h_{m, n} D_{1}^{m} D_{2}^{n} x^{m} y^{n}\right)^{-1}$ and of $\operatorname{LTR} \prod_{\substack{q=1 \\ m+n=q}}^{\infty}\left(I-h_{m, n} A^{m+n} x^{m} y^{n}\right)^{-1}$.

\subsection{Illustrative examples}

In this section various examples are given to illustrate the main theorems of the previous sections. Our first example is an extension of Theorem 4.4.1.

Example 4.6.1. Consider the following special series $F(x, y)$ with the special related majorant matrix functions series:

$$
\begin{aligned}
& F(x, y)=I+\sum_{\substack{p=1 \\
u+v=p}}^{\infty} A_{u \alpha+\beta, v \alpha+\beta} x^{u \alpha+\beta} y^{v \alpha+\beta}=L T R \prod_{\substack{q=1 \\
m+n=q}}^{\infty}\left(I-H_{m, n} x^{m} y^{n}\right)^{-1} \\
& C(x, y)=I-\sum_{\substack{p=1 \\
u+v=p}}^{\infty}\left|A_{u \alpha+\beta, v \alpha+\beta}\right| x^{u \alpha+\beta} y^{v \alpha+\beta}=L T R \prod_{\substack{q=1 \\
m+n=q}}^{\infty}\left(I+S_{m, n} x^{m} y^{n}\right)^{-1} \\
& M(x, y)=I-\sum_{\substack{p=1 \\
u+v=p}}^{\infty} s^{\alpha(u+v)+2 \beta} W x^{u \alpha+\beta} y^{v \alpha+\beta}=L T R \prod_{\substack{q=1 \\
m+n=q}}^{\infty}\left(I+R_{m, n} x^{m} y^{n}\right)^{-1},
\end{aligned}
$$

where $\alpha, \beta \in \mathbb{N}_{0}, W \in M_{d}(\mathbb{C})$, and it is defined as

$$
W=\left(w_{u, v}\right), \quad \text { where } \quad w_{u, v}=1, \quad v, u=1, \ldots, d .
$$

Furthermore, given $A_{u \alpha+\beta, v \alpha+\beta} \in M_{d}(\mathbb{C})$ where $A_{u \alpha+\beta, v \alpha+\beta}=\left(a_{\psi, \omega}(u \alpha+\beta, v \alpha+\beta)\right)$ for $\psi, \omega=1, \ldots, d$, define

$$
a(u \alpha+\beta, v \alpha+\beta):=\max _{\psi, \omega}\left|a_{\psi, \omega}(u \alpha+\beta, v \alpha+\beta)\right|,
$$


then $s$ is defined as

$$
s:=\sup _{\substack{\alpha, \beta \in \mathbb{N}_{0} \\ m+n \geq 1}}[a(u \alpha+\beta, v \alpha+\beta)]^{\frac{1}{\alpha(u+v)+2 \beta}} .
$$

Next by requiring $|x|<\frac{1}{s}$ and that $|y|<\frac{1}{s}$, we can derive the closed form of the left hand side of Equation (4.104)

$$
\begin{aligned}
M(x, y) & =I-W \sum_{\substack{p=1 \\
u+v=p}}^{\infty} s^{\alpha(u+v)+2 \beta} x^{u \alpha+\beta} y^{v \alpha+\beta} \\
& =I-\left(s^{2} x y\right)^{\beta} W \sum_{\substack{p=1 \\
u+v=p}}^{\infty} s^{\alpha(u+v)} x^{u \alpha} y^{v \alpha} \\
& =I-\left(s^{2} x y\right)^{\beta} W\left[\sum_{u=0}^{\infty}(s x)^{u \alpha} \sum_{v=0}^{\infty}(s y)^{v \alpha}-1\right] \\
& =I-\left(s^{2} x y\right)^{\beta} W\left[\frac{1}{1-(s x)^{\alpha}} \cdot \frac{1}{1-(s y)^{\alpha}}-1\right] \\
& =I-\frac{W\left(s^{2} x y\right)^{\beta}\left[s^{\alpha}\left(x^{\alpha}+y^{\alpha}\right)-\left(s^{2} x y\right)^{\alpha}\right]}{\left[1-(s x)^{\alpha}\right]\left[1-(s y)^{\alpha}\right]} \\
& =\frac{I-\left[I+\left(s^{2} x y\right)^{\beta} W\right]\left[s^{\alpha}\left(x^{\alpha}+y^{\alpha}\right)-\left(s^{2} x y\right)^{\alpha}\right]}{\left[1-(s x)^{\alpha}\right]\left[1-(s y)^{\alpha}\right]} \\
& =L T R \prod_{\substack{q=1 \\
m+n=q}}^{\infty}\left(I+R_{m, n} x^{n} y^{n}\right)^{-1} .
\end{aligned}
$$

To obtain the domain of inverse convergence as defined in Definition 4.4.1, the straightforward computation in Equation (4.105) shows that eigenvalues of $M(x, y)$ are 1 and $\frac{1-\left[1+d\left(s^{2} x y\right)^{\beta}\right]\left[s^{\alpha}\left(x^{\alpha}+y^{\alpha}\right)-\left(s^{2} x y\right)^{\alpha}\right]}{\left[1-(s x)^{\alpha}\right]\left[1-(s y)^{\alpha}\right]}$. As a result, the eigenvalues of $M(x, y)^{-1}$ are 1 and

$\frac{\left[1-(s x)^{\alpha}\right]\left[1-(s y)^{\alpha}\right]}{1-\left[1+d\left(s^{2} x y\right)^{\beta}\right]\left[s^{\alpha}\left(x^{\alpha}+y^{\alpha}\right)-\left(s^{2} x y\right)^{\alpha}\right]}$. Note that $\frac{\left[1-(s x)^{\alpha}\right]\left[1-(s y)^{\alpha}\right]}{1-\left[1+d\left(s^{2} x y\right)^{\beta}\right]\left[s^{\alpha}\left(x^{\alpha}+y^{\alpha}\right)-\left(s^{2} x y\right)^{\alpha}\right]}=0$ or $\infty$ if and only if $(s x)^{\alpha}=1$ or $(s y)^{\alpha}=1$, or $1-\left[1+d\left(s^{2} x y\right)^{\beta}\right]\left[s^{\alpha}\left(x^{\alpha}+y^{\alpha}\right)-\left(s^{2} x y\right)^{\alpha}\right]=0$. Thus the nearest singularities of $M(x, y)^{-1}$ to $(x, y)=(0,0)$ are the the points $(x, y)$ satisfy the equation $1-\left[1+d\left(s^{2} x y\right)^{\beta}\right]\left[s^{\alpha}\left(x^{\alpha}+y^{\alpha}\right)-\left(s^{2} x y\right)^{\alpha}\right]=0$, which means that $M(x, y)^{-1}=$ $R T L \prod_{q=1}^{\infty}\left(I+R_{m, n} x^{m} y^{n}\right)$ is absolutely convergent (in the inverse sense) in $q=1$
$m+n=q$

$$
\mathbb{D}_{1}=\left\{(x, y) \in \mathbb{C}^{2}:\left[1+\left|d\left(s^{2} x y\right)^{\beta}\right|\right]\left[s^{\alpha}\left(|x|^{\alpha}+|y|^{\alpha}\right)+\left|\left(s^{2} x y\right)\right|^{\alpha}\right]<1\right\}
$$


Since $O \leq\left|H_{m, n}\right| \leq S_{m, n} \leq R_{m, n}$, Proposition 4.4.1 implies that $F(x, y)^{-1}$ and $C(x, y)^{-1}$ are also well defined and absolutely convergent over domain $\mathbb{D}_{1}$.

Note that if $\alpha=1, \beta=0$, then the domain $\mathbb{D}_{1}$ defining in Theorem 4.4.1 [(2.)] is a special case of $\mathbb{D}_{1}$ defining in (4.106).

In order to investigate the convergence of $L T R \prod_{\substack{q=1 \\ m+n=q}}^{\infty}\left(I-H_{m, n} x^{m} y^{n}\right)^{-1}$ in the sense of Definition 4.4.4, we define a majorant of $M(x, y)$, namely

$$
\begin{aligned}
& \hat{M}(x, y)=I-W \sum_{q=1}^{\infty}\left(s^{2} x y\right)^{\beta}\left[(s x)^{\alpha}+(s y)^{\alpha}\right]^{q} \\
& =I-W \sum_{q=1}^{\infty} \sum_{k=0}^{q}\left(s^{2} x y\right)^{\beta}\left(\begin{array}{l}
q \\
k
\end{array}\right)\left[(s x)^{\alpha}\right]^{q-k}\left[(s y)^{\alpha}\right]^{k} \quad \text { Set } u=q-k \text { and } v=k \\
& =I-W \sum_{\substack{p=1 \\
u+v=p}}^{\infty}\left(\begin{array}{c}
u+v \\
v
\end{array}\right) s^{\alpha(u+v)+2 \beta} x^{\alpha u+\beta} y^{\alpha v+\beta} \\
& =I-W \sum_{\substack{p=1 \\
u+v=p}}^{\infty} \hat{M}_{\alpha u+\beta, \alpha v+\beta} x^{\alpha u+\beta} y^{\alpha v+\beta} \\
& =\operatorname{LTR} \prod_{\substack{q=1 \\
u+v=q}}^{\infty}\left(I+\hat{R}_{m, n} x^{m} y^{n}\right)^{-1}
\end{aligned}
$$

where $\hat{M}_{\alpha u+\beta, \alpha v+\beta}=\left(\begin{array}{c}u+v \\ v\end{array}\right) s^{\alpha(u+v)+2 \beta}$.

Since

$$
M_{\alpha u+\beta, \alpha v+\beta}=s^{\alpha(u+v)+2 \beta} W \leq\left(\begin{array}{c}
u+v \\
v
\end{array}\right) s^{\alpha(u+v)+2 \beta} W=\hat{M}_{\alpha u+\beta, \alpha v+\beta},
$$

then again by the structure property we have

$$
O \leq\left|H_{m, n}\right| \leq R_{m, n} \leq \hat{R}_{m, n} .
$$

Next we invert $\hat{M}(x, y)$ since this inversion will provide upper bound for $\hat{R}_{m, n}$. In particular, we have

$$
\begin{aligned}
{[\hat{M}(x, y)]^{-1} } & =I+\sum_{\substack{p=1 \\
m+n=p}}^{\infty} \tilde{M}_{m, n} x^{n} y^{n} \\
& =R T L \prod_{\substack{q=1 \\
m+n=q}}^{\infty}\left(I+\hat{R}_{m, n} x^{m} y^{n}\right) .
\end{aligned}
$$


Since $\left(\hat{R}_{m, n}\right)_{u, v=0}^{\infty}$ is a sequence of positive matrices, then both $R T L \prod_{\substack{p=1 \\ m+n=p}}^{\infty}\left(I+\hat{R}_{m, n} x^{m} y^{n}\right)$ and $I+\sum_{\substack{p=1 \\ m+n=p}}^{\infty} \tilde{M}_{m, n} x^{m} y^{n}$ have the same domain of convergence. Furthermore, coefficients compassion shows that

$$
O \leq \hat{R}_{m, n} \leq \tilde{M}_{m, n}
$$

Combining Equations (4.108), and (4.109) together implies that

$$
\begin{aligned}
& \left|\sum_{\substack{p=1 \\
m+n=p}}^{\infty} \sum_{k=1}^{\infty}\left(H_{m, n} x^{m} y^{n}\right)^{k}\right| \leq \sum_{\substack{p=1 \\
m+n=p}}^{\infty} \sum_{k=1}^{\infty}\left|H_{m, n}\right|^{k}|x|^{m k}|y|^{n k} \\
& \leq \sum_{\substack{p=1 \\
m+n=p}}^{\infty} \sum_{k=1}^{\infty} \hat{R}_{m, n}^{k}|x|^{m k}|y|^{n k} \\
& \leq \sum_{\substack{p=1 \\
m+n=p}}^{\infty} \sum_{k=1}^{\infty} \hat{M}_{m, n}^{k}|x|^{m k}|y|^{n k}
\end{aligned}
$$

Equation (4.110) shows that we need to determine an upper bound for $\tilde{M}_{m, n}$. To find the desired upper bound, we consider special case for $\alpha$ and $\beta$.

Case 1: Set $\alpha \in \mathbb{N}$ and $\beta=0$ in Equation (4.107), then we have

$$
\begin{aligned}
\hat{M}(x, y) & =I-W \sum_{q=1}^{\infty}\left[(s x)^{\alpha}+(s y)^{\alpha}\right]^{q}=I-\frac{(s x)^{\alpha}+(s y)^{\alpha}}{1-\left[(s x)^{\alpha}+(s y)^{\alpha}\right]} W \\
& =I-\sum_{\substack{r=1 \\
u+v=r}}^{\infty} \hat{M}_{\alpha u, \alpha v} x^{\alpha u} y^{\alpha v}, \text { where } \hat{M}_{\alpha u, \alpha v}=W\left(\begin{array}{c}
u+v \\
v
\end{array}\right) s^{\alpha(u+v) .}
\end{aligned}
$$

We will assume that

$$
\left|\left[(s x)^{\alpha}+(s y)^{\alpha}\right]\right|<\left|(d+1)\left[(s x)^{\alpha}+(s y)^{\alpha}\right]\right|<\rho<1 .
$$

Then

$$
\left|\frac{(s x)^{\alpha}+(s y)^{\alpha}}{1-\left[(s x)^{\alpha}+(s y)^{\alpha}\right]}\right| \leq \frac{\left|(s x)^{\alpha}+(s y)^{\alpha}\right|}{|1-|(s x)^{\alpha}+(s y)^{\alpha}||} \leq \frac{1}{1-\rho} .
$$

So if we further require $\|W\|<1-\rho$, we find that

$$
[\hat{M}(x, y)]^{-1}=I+\sum_{\substack{r=1 \\ u+v=r}}^{\infty} \hat{M}_{u \alpha+\beta, v \alpha+\beta} x^{u \alpha+\beta} y^{v \alpha+\beta}
$$




$$
\begin{aligned}
& =\left[I-\frac{W\left[(s x)^{\alpha}+(s y)^{\alpha}\right]}{1-\left[(s x)^{\alpha}+(s y)^{\alpha}\right]}\right]^{-1} \\
& =I+\sum_{n=1}^{\infty}\left[\frac{(s x)^{\alpha}+(s y)^{\alpha}}{1-\left[(s x)^{\alpha}+(s y)^{\alpha}\right]}\right]^{n} W^{n} \\
& =I+\frac{W}{d} \sum_{n=1}^{\infty}\left[\frac{d\left[(s x)^{\alpha}+(s y)^{\alpha}\right]}{1-\left[(s x)^{\alpha}+(s y)^{\alpha}\right]}\right]^{n} \\
& =I+\frac{W\left[(s x)^{\alpha}+(s y)^{\alpha}\right]}{1-\left[(s x)^{\alpha}+(s y)^{\alpha}\right]}\left[1-\frac{d\left[(s x)^{\alpha}+(s y)^{\alpha}\right]}{1-\left[(s x)^{\alpha}+(s y)^{\alpha}\right]}\right]^{-1} \\
& =I+\frac{\left[(s x)^{\alpha}+(s y)^{\alpha}\right] W}{1-[d+1]\left[(s x)^{\alpha}+(s y)^{\alpha}\right]} \\
& =I+\left[(s x)^{\alpha}+(s y)^{\alpha}\right] W \sum_{k=0}^{\infty}\left([d+1]\left[(s x)^{\alpha}+(s y)^{\alpha}\right]\right)^{k} \\
& =I+W \sum_{k=1}^{\infty}[d+1]^{k-1}\left[(s x)^{\alpha}+(s y)^{\alpha}\right]^{k} \\
& =I+W \sum_{k=1}^{\infty}[d+1]^{k-1}\left[(s y)^{\alpha}\right]^{k}+ \\
& W \sum_{k=1}^{\infty}[d+1]^{k-1} \sum_{r=1}^{k}\left(\begin{array}{l}
k \\
r
\end{array}\right)\left[(s x)^{\alpha}\right]^{r}\left[(s y)^{\alpha}\right]^{k-r} \\
& =I+W \sum_{k=1}^{\infty}[d+1]^{k-1}\left[(s y)^{\alpha}\right]^{k}+ \\
& W \sum_{k=1}^{\infty}[d+1]^{k-1} \sum_{r=1}^{k}\left(\begin{array}{l}
k \\
r
\end{array}\right)\left[(s x)^{\alpha}\right]^{r}\left[(s y)^{\alpha}\right]^{k-r} \\
& =I+W \sum_{k=1}^{\infty}[d+1]^{k-1}\left[(s y)^{\alpha}\right]^{k}+ \\
& W \sum_{r=1}^{\infty} \sum_{k=r}^{\infty}[d+1]^{k-1}\left(\begin{array}{l}
k \\
r
\end{array}\right)\left[(s x)^{\alpha}\right]^{r}\left[(s y)^{\alpha}\right]^{k-r} \\
& =I+W \sum_{\substack{r=1 \\
u+v=r}}^{\infty}[d+1]^{u+v-1} s^{\alpha(u+v)}\left(\begin{array}{c}
u+v \\
u
\end{array}\right) x^{\alpha u} y^{\alpha \nu} \\
& =I+W \sum_{\substack{r=1 \\
u+v=r}}^{\infty} \tilde{M}_{\alpha u, \alpha v} x^{\alpha u} y^{\alpha v}
\end{aligned}
$$

where $\tilde{M}_{\alpha u, \alpha v}=[d+1]^{u+v-1} s^{\alpha(u+v)}\left(\begin{array}{c}u+v \\ u\end{array}\right) W$. 
Furthermore, we have

$$
\tilde{M}_{\alpha u, \alpha v}=[d+1]^{u+v-1} s^{\alpha(u+v)}\left(\begin{array}{c}
u+v \\
u
\end{array}\right) W \leq[d+1]^{u+v-1}\left(2 s^{\alpha}\right)^{u+v} W .
$$

Plugging the desired upper bound of (4.115) into (4.110), we find that

$$
\begin{aligned}
& \left|\sum_{\substack{p=1 \\
m+n=p}}^{\infty} \sum_{k=1}^{\infty}\left(H_{m, n} x^{m} y^{n}\right)^{k}\right| \leq \sum_{\substack{r=1 \\
u+v=r}}^{\infty} \sum_{k=1}^{\infty}\left(\tilde{M}_{\alpha u, \alpha v}\right)^{k}|x|^{\alpha u k}|y|^{\alpha v k} \\
& \leq \sum_{\substack{r=1 \\
u+v=r}}^{\infty} \sum_{\substack{k=1 \\
\infty}}^{\infty} \frac{\left[2(d+1) s^{\alpha}\right]^{k(u+v)}}{(d+1)^{k}} W^{k}|x|^{\alpha u k}|y|^{\alpha v k} \\
& \leq \sum_{\substack{r=1 \\
u+v=r}}^{\infty} \sum_{k=1}^{\infty} \frac{\left[2(d+1) s^{\alpha}\right]^{k(u+v)}}{(d+1)^{k}}\left[d^{k-1} W\right]|x|^{\alpha u k}|y|^{\alpha v k} \\
& \leq W \sum_{\substack{r=1 \\
u+v=r}}^{\infty} \sum_{\substack{k=1 \\
\leq}} \frac{\left[2(d+1) s^{\alpha}\right]^{k(u+v)}}{(d+1)^{k}}(d+1)^{k-1}|x|^{\alpha u k}|y|^{\alpha v k} \\
& \leq \frac{W}{(d+1)} \sum_{\substack{r=1 \\
u+v=r}}^{\infty} \sum_{k=1}^{\infty}\left[\left[2 s^{\alpha}(d+1)\right]^{u+v}|x|^{\alpha u}|y|^{\alpha v}\right]^{k} \\
& =\frac{W}{(d+1)} \sum_{\substack{r=1 \\
u+v=r}}^{\infty} \frac{\left[2 s^{\alpha}(d+1)\right]^{(u+v)}|x|^{\alpha u}|y|^{\alpha v}}{1-\left[2 s^{\alpha}(d+1)|x|^{\alpha}\right] u\left[2 s^{\alpha}(d+1)|y|^{\alpha}\right]^{v}},
\end{aligned}
$$

where in the penultimate line we assumed assumed that $2 s^{\alpha}(d+1)|x|^{\alpha}<\rho_{1}<\rho<1$ and that $2 s^{\alpha}(d+1)|y|^{\alpha}<\rho_{2}<\rho<1$. When working over these refined polydiscs, the previous inequity becomes

$$
\begin{aligned}
& \left|\sum_{\substack{p=1 \\
m+n=p}}^{\infty} \sum_{k=1}^{\infty}\left(H_{m, n} x^{m} y^{n}\right)^{k}\right| \leq \frac{W}{(d+1)} \sum_{\substack{r=1 \\
u+v=r}}^{\infty} \frac{\left[2 s^{\alpha}(d+1)|x|^{\alpha}\right]^{u}\left[2 s^{\alpha}(d+1)|y|^{\alpha}\right]^{u}}{1-\rho^{2}} \\
& \leq \frac{W}{(d+1)(1-\rho)} \sum_{\substack{r=1 \\
u+v=r}}^{\infty}\left[2 s^{\alpha}(d+1)|x|^{\alpha}\right]^{u}\left[2 s^{\alpha}(d+1)|y|^{\alpha}\right]^{v} \\
& \leq \frac{W}{(d+1)(1-\rho)} \sum_{\substack{r=1 \\
u+v=r}}^{\infty} \rho^{u+v} \\
& =\frac{W}{(d+1)(1-\rho)}\left[\sum_{u=0}^{\infty} \rho^{u} \sum_{v=0}^{\infty} \rho^{v}-1\right]
\end{aligned}
$$




$$
\begin{aligned}
& =\frac{W}{(d+1)(1-\rho)}\left[\frac{1}{(1-\rho)^{2}}-1\right] \\
& =\frac{\left[2 \rho-\rho^{2}\right] W}{(d+1)(1-\rho)^{3}} .
\end{aligned}
$$

In conclusion, by using a majorant of a majorant and clever use of inversion, we finally showed

$$
\begin{aligned}
\left|\sum_{\substack{p=1 \\
m+n=p}}^{\infty} \sum_{k=1}^{\infty}\left(H_{m, n} x^{m} y^{n}\right)^{k}\right| & \leq \sum_{\substack{r=1 \\
u+v=r}}^{\infty} \sum_{k=1}^{\infty}\left(\tilde{M}_{\alpha u, \alpha v}\right)^{k}|x|^{\alpha u k}|y|^{\alpha v k} \\
& =\frac{\left[2 \rho-\rho^{2}\right] W}{(d+1)(1-\rho)^{3}}
\end{aligned}
$$

whenever $\left[2 s^{\alpha}(d+1)\right]|x|^{\alpha}<\rho<1$ and $\left[2 s^{\alpha}(d+1)\right]|y|^{\alpha}<\rho<1$. Therefore, an estimate for domain of absolute convergence of $L T R \prod_{\substack{q=1 \\ m+n=q}}^{\infty}\left(I+\hat{R}_{m, n} x^{m} y^{n}\right)^{-1}$ is

$$
\mathbb{D}_{2}=\left\{(x, y) \in \mathbb{C}^{2}:|x|<\frac{1}{[2(d+1)]^{\frac{1}{\alpha}} S} \text { and }|y|<\frac{1}{[2(d+1)]^{\frac{1}{\alpha}} S}\right\} .
$$

Since $O \leq\left|H_{m, n}\right| \leq S_{m, n} \leq R_{m, n} \leq \hat{R}_{m, n}$, Definition 4.4.4 implies that $\mathbb{D}_{2}$ is also an estimate for the domain of absolute convergence of $\operatorname{LTR} \prod_{\substack{q=1 \\ m+n=q}}^{\infty}\left(I-H_{m, n} x^{m} y^{n}\right)^{-1}$ and of $\operatorname{LTR} \prod_{\substack{q=1 \\ m+n=q}}^{\infty}\left(I+S_{m, n} x^{m} y^{n}\right)^{-1}$.

Note that if we assume $\alpha=1$ and $\beta=0$, then the domain $\mathbb{D}_{2}$ defining in Theorem 4.4.1 [(3.)] is a special case of the domain $\mathbb{D}_{2}$, which is defining in (4.119).

Case2: For simplicity, we set $\alpha=0$ and $\beta \in \mathbb{N}$ in Equation (4.104), then we have

$$
M(x, y)=I-W\left(s^{2} x y\right)^{\beta}=L T R \prod_{\substack{q=1 \\ m+n=q}}^{\infty}\left(I+R_{m, n} x^{m} y^{n}\right)^{-1} .
$$

We will assume that $\left|\left(s^{2} x y\right)^{\beta}\right|<\rho<1$, and further we require $\|W\|\left|\left(s^{2} x y\right)^{\beta}\right|<\|W\| \rho<1$, i.e. $\|W\|<\frac{1}{\rho}$. Then we have

$$
\begin{aligned}
& M^{-1}(x, y)=\left[I-W\left(s^{2} x y\right)^{\beta}\right]^{-1}=I+\sum_{k=1}^{\infty}\left[W\left(s^{2} x y\right)^{\beta}\right]^{k} \\
& =I+\frac{W}{d} \sum_{k=1}^{\infty}\left[d\left(s^{2} x y\right)^{\beta}\right]^{k}
\end{aligned}
$$




$$
\begin{aligned}
& =I+\frac{W}{d} \frac{1}{1-d\left(s^{2} x y\right)^{\beta}}, \quad \text { which means that }\left|d\left(s^{2} x y\right)^{\beta}\right|<\rho_{1}<\rho<1 \\
& =I+\sum_{k=1}^{\infty} \tilde{M}_{\beta k, \beta k} x^{\beta k} y^{\beta k} \\
& =R T L \prod_{\substack{q=1 \\
m+n=q}}^{\infty}\left(I+R_{m, n} x^{m} y^{n}\right)
\end{aligned}
$$

where $\tilde{M}_{\beta k, \beta k}=d^{k-1} s^{2 k \beta} W$

Note that the penultimate line in Equation (4.120) shows that $\beta k=m$ and $\beta k=n$, and this will only the nonzero $\tilde{M}_{m, n}$.

Now we repeat inequality chain at (4.110). Since $R_{m, n} \leq \tilde{M}_{m, n}$, then we obtain

$$
\begin{aligned}
& \left.\sum_{\substack{p=1 \\
m+n=p}}^{\infty} \sum_{k=1}^{\infty}\left|R_{m, n}\right|^{k}|x|^{m k}|y|^{n k}\right|^{k} \leq \sum_{\substack{q=1 \\
m+n=q}}^{\infty} \sum_{k=1}^{\infty}\left|\hat{M}_{m, n}\right|^{k}|x|^{m k}|y|^{n k} \\
= & \sum_{q=1}^{\infty} \sum_{k=1}^{\infty}\left|\hat{M}_{\beta q, \beta q}\right|^{k}|x|^{\beta q k}|y|^{\beta q k} \\
= & \sum_{q=1}^{\infty} \sum_{k=1}^{\infty}\left[d^{q-1} s^{2 q \beta} W\right]^{k}|x|^{\beta q k}|y|^{\beta q k} \\
= & \frac{W}{d} \sum_{q=1}^{\infty} \sum_{k=1}^{\infty}\left[d^{q} s^{2 q \beta}\right]^{k}|x|^{\beta q k}|y|^{\beta q k} \\
\leq & \frac{W}{d} \sum_{q=1}^{\infty} \sum_{k=1}^{\infty}\left[d s^{2 \beta}\right]^{k q}|x|^{\beta q k}|y|^{\beta q k}, \\
\leq & \frac{W}{d} \sum_{q=1}^{\infty} \sum_{k=1}^{\infty} d^{2 k q}|s x|^{\beta q k}|s y|^{\beta q k}, \\
= & \frac{W}{d} \sum_{q=1}^{\infty} \sum_{k=1}^{\infty}\left[d|s x|^{\beta}\right]^{q k}\left[d|s y|^{\beta}\right]^{q k} .
\end{aligned}
$$

Now we assume $d|s x|^{\beta}<\rho_{1}<\rho<1, d|s y|^{\beta}<\rho_{2}<\rho<1$, then we have

$$
\begin{aligned}
\left.\sum_{\substack{p=1 \\
m+n=p}}^{\infty} \sum_{k=1}^{\infty}\left|R_{m, n}\right|^{k}|x|^{m k}|y|^{n k}\right|^{k} & \leq \frac{W}{d} \sum_{q=1}^{\infty} \sum_{k=1}^{\infty}\left[\rho^{2}\right]^{q k} \\
& =\frac{W}{d} \sum_{q=1}^{\infty} \frac{\rho^{2 q}}{1-\rho^{2 q}} \\
& \leq \frac{W}{d(1-\rho)} \sum_{q=1}^{\infty} \rho^{2 q}=\frac{W \rho^{2}}{d(1-\rho)\left(1-\rho^{2}\right)}
\end{aligned}
$$


Therefore, an estimate for domain of absolute convergence of $\operatorname{LTR} \prod_{\substack{q=1 \\ m+n=q}}^{\infty}\left(I+R_{m, n} x^{m} y^{n}\right)$ is

$$
\mathbb{D}_{2}=\left\{(x, y) \in \mathbb{C}^{2}:|x|<\frac{1}{s d^{\frac{1}{\beta}}} \text { and }|y|<\frac{1}{s d^{\frac{1}{\beta}}}\right\} .
$$

Since $O \leq\left|H_{m, n}\right| \leq S_{m, n} \leq R_{m, n}$, Definition 4.4 .4 implies that $\mathbb{D}_{2}$ is also an estimate for the domain of absolute convergence of $\operatorname{LTR} \prod_{\substack{q=1 \\ m+n=q}}^{\infty}\left(I-H_{m, n} x^{m} y^{n}\right)^{-1}$ and of $\operatorname{LTR} \prod_{\substack{q=1 \\ m+n=q}}^{\infty}(I+$ $\left.S_{m, n} x^{m} y^{n}\right)^{-1}$.

Case3: Let $\alpha, \beta \in \mathbb{N}$, then we have

$$
\hat{M}(x, y)=I-W \sum_{q=1}^{\infty}\left(s^{2} x y\right)^{\beta}\left[(s x)^{\alpha}+(s y)^{\alpha}\right]^{q}=I-\frac{W\left(s^{2} x y\right)^{\beta}\left[(s x)^{\alpha}+(s y)^{\alpha}\right]}{1-\left[(s x)^{\alpha}+(s y)^{\alpha}\right]} .
$$

We will assume that

$$
\left|\left(s^{2} x y\right)^{\beta}\left[(s x)^{\alpha}+(s y)^{\alpha}\right]\right|<\left|\left[d\left(s^{2} x y\right)^{\beta}+1\right]\left[(s x)^{\alpha}+(s y)^{\alpha}\right]\right|<\rho<1 .
$$

Then

$$
\left|\frac{\left(s^{2} x y\right)^{\beta}\left[(s x)^{\alpha}+(s y)^{\alpha}\right]}{1-\left[(s x)^{\alpha}+(s y)^{\alpha}\right]}\right| \leq \frac{\left|\left(s^{2} x y\right)^{\beta}\left[(s x)^{\alpha}+(s y)^{\alpha}\right]\right|}{|1-|(s x)^{\alpha}+(s y)^{\alpha}||} \leq \frac{1}{1-\rho} .
$$

So if we further require $\|W\|<1-\rho$, we find that

$$
\begin{aligned}
& {[\hat{M}(x, y)]^{-1}=I+\sum_{\substack{p=1 \\
u+v=p}}^{\infty} \hat{M}_{u \alpha+\beta, v \alpha+\beta} x^{u \alpha+\beta} y^{v \alpha+\beta}} \\
& =\left[I-\frac{W\left(s^{2} x y\right)^{\beta}\left[(s x)^{\alpha}+(s y)^{\alpha}\right]}{\mid 1-\left[(s x)^{\alpha}+(s y)^{\alpha}\right]}\right]^{-1} \\
& =I+\sum_{n=1}^{\infty}\left[\frac{\left(s^{2} x y\right)^{\beta}\left[(s x)^{\alpha}+(s y)^{\alpha}\right]}{1-\left[(s x)^{\alpha}+(s y)^{\alpha}\right]}\right]^{n} W^{n} \\
& =I+\frac{W}{d} \sum_{n=1}^{\infty}\left[\frac{d\left(s^{2} x y\right)^{\beta}\left[(s x)^{\alpha}+(s y)^{\alpha}\right]}{1-\left[(s x)^{\alpha}+(s y)^{\alpha}\right]}\right]^{n} \\
& =I+\frac{W\left(s^{2} x y\right)^{\beta}\left[(s x)^{\alpha}+(s y)^{\alpha}\right]}{1-\left[(s x)^{\alpha}+(s y)^{\alpha}\right]}\left[1-\frac{d\left(s^{2} x y\right)^{\beta}\left[(s x)^{\alpha}+(s y)^{\alpha}\right]}{1-\left[(s x)^{\alpha}+(s y)^{\alpha}\right]}\right]^{-1}
\end{aligned}
$$




$$
\begin{aligned}
= & I+\frac{\left(s^{2} x y\right)^{\beta}\left[(s x)^{\alpha}+(s y)^{\alpha}\right] W}{1-\left[d\left(s^{2} x y\right)^{\beta}+1\right]\left[(s x)^{\alpha}+(s y)^{\alpha}\right]} \\
= & I+\left(s^{2} x y\right)^{\beta}\left[(s x)^{\alpha}+(s y)^{\alpha}\right] W \sum_{k=0}^{\infty}\left(\left[d\left(s^{2} x y\right)^{\beta}+1\right]\left[(s x)^{\alpha}+(s y)^{\alpha}\right]\right)^{k} \\
= & I+\left(s^{2} x y\right)^{\beta}\left[(s x)^{\alpha}+(s y)^{\alpha}\right] W \sum_{k=0}^{\infty} \sum_{i+j+p+q=k}\left(\begin{array}{c}
k \\
i j p q
\end{array}\right)(s x)^{\alpha i}(s y)^{\alpha j} \times \\
& \left(d s^{2 \beta+\alpha} x^{\beta+\alpha} y^{\beta}\right)^{p}\left(d s^{2 \beta+\alpha} x^{\beta} y^{\alpha+\beta}\right)^{q} \\
= & I+\left[x^{\alpha+\beta} y^{\beta}+x^{\beta} y^{\alpha+\beta}\right] s^{\alpha+2 \beta} W \sum_{k=0}^{\infty} s^{\alpha k} \sum_{i+j+p+q=k}\left(\begin{array}{c}
k \\
i j p q
\end{array}\right) s^{2 \beta(p+q)} \times \\
& d^{p+q} x^{\alpha(i+p)+\beta(p+q)} y^{\alpha(j+q)+\beta(p+q)} \\
= & I+S_{1}+S_{2},
\end{aligned}
$$

where

$$
S_{1}=s^{\alpha+2 \beta} W \sum_{k=0}^{\infty} s^{\alpha k} \sum_{i+j+p+q=k}\left(\begin{array}{c}
k \\
i j p q
\end{array}\right) s^{2 \beta(p+q)} d^{p+q} x^{\alpha(i+p+1)+\beta(p+q+1)} y^{\alpha(j+q)+\beta(p+q+1)},
$$

and

$$
S_{2}=s^{\alpha+2 \beta} W \sum_{k=0}^{\infty} s^{\alpha k} \sum_{i+j+p+q=k}\left(\begin{array}{c}
k \\
i j p q
\end{array}\right) s^{2 \beta(p+q)} d^{p+q} x^{\alpha(i+p)+\beta(p+q+1)} y^{\alpha(j+q+1)+\beta(p+q+1)}
$$

From $S_{1}$ we set

$$
\begin{aligned}
& u=i+p+1, w=p+q+1, v=j+q, \text { then we have } u+v=k+1 . \text { Also, } \\
& i+j=u+v-w .
\end{aligned}
$$

So, $k=u+v-1$.

Hence, the coefficient of $x^{\alpha u+\beta w} y^{\alpha v+\beta w}$ in $S_{1}$ is

$$
\begin{aligned}
& s^{\alpha+2 \beta} s^{\alpha(u+v-1)}\left(\begin{array}{c}
u+v-1 \\
i j u-i-1 v-j
\end{array}\right) s^{2 \beta(w-1)} d^{w-1} W \\
& =s^{2 \beta w+\alpha(u+v)} d^{w-1}\left(\begin{array}{c}
u+v-1 \\
i j u-i-1 \\
j u-j
\end{array}\right) W \\
& \leq(u+v-w+1) s^{2 \beta w+\alpha(u+v)} d^{w-1} 2^{u+v-1} W \\
& \leq s^{2 \beta w+\alpha(u+v)} d^{w-1} 2^{2(u+v)-w} W
\end{aligned}
$$

From $S_{2}$ we set

$$
k=u+v-1, i+j=u+v-w ; \text { with } u=i+p, w=p+q+1, v=j+q+1
$$


Hence, the coefficient of $x^{\alpha u+\beta w} y^{\alpha v+\beta w}$ in $S_{2}$ is

$$
\begin{aligned}
& s^{\alpha(u+v)+2 \beta w} d^{w-1}\left(\begin{array}{c}
u+v-1 \\
i j u-i v-j-1
\end{array}\right) W, \quad \text { subject to } i+j=u+v-w \\
& \leq s^{\alpha(u+v)+2 \beta w} d^{w-1} 2^{2(u+v)-w} W .
\end{aligned}
$$

Therefore, we have

$$
[\tilde{M}(x, y)]^{-1}=I+\sum_{\substack{r=1 \\ u+v=r}}^{\infty} \sum_{w=1}^{\infty} \tilde{M}_{\alpha u+\beta w, \alpha v+\beta w} x^{\alpha u+\beta w} y^{\alpha v+\beta w},
$$

and Inequalities (4.126), (4.127) show that

$$
\tilde{M}_{\alpha u+\beta w, \alpha v+\beta w} \leq s^{\alpha(u+v)+2 \beta w} d^{w-1} 2^{2(u+v)-w} W .
$$

Plugging the desired upper bound of (4.129) into (4.110), we find that

$$
\begin{aligned}
& \left|\sum_{\substack{p=1 \\
m+n=p}}^{\infty} \sum_{k=1}^{\infty}\left(H_{m, n} x^{m} y^{n}\right)^{k}\right|=\left|\sum_{\substack{r=1 \\
u+v=r}}^{\infty} \sum_{w=1}^{\infty} \sum_{k=1}^{\infty}\left[\tilde{M}_{\alpha u+\beta w, \alpha v+\beta w} x^{\alpha u+\beta w} y^{\alpha v+\beta w}\right]^{k}\right| \\
& =\left|\sum_{\substack{r=1 \\
u+v=r}}^{\infty} \sum_{\substack{w=1 \\
W}}^{\infty}\left[s^{\alpha(u+v)+2 \beta w} d^{w-1} 2^{2(u+v)-w} W x^{\alpha u+\beta w} y^{\alpha v+\beta w}\right]^{k}\right| \\
& \leq \frac{W}{d} \sum_{\substack{r=1 \\
u+v=r}}^{\infty} \sum_{w=1}^{\infty} \sum_{k=1}^{\infty}\left[s^{\alpha(u+v)+2 \beta w} d^{w} 2^{2(u+v)-w}|x|^{\alpha u+\beta w}|y|^{\alpha v+\beta w}\right]^{k} \text { since } W^{k}=d^{k-1} W \\
& =\frac{W}{d} \sum_{\substack{r=1 \\
u+v=r}}^{\infty} \sum_{w=1}^{\infty} \sum_{k=1}^{\infty}\left[d^{w} 2^{2(u+v)-w}|s x|^{\alpha u+\beta w}|s y|^{\alpha v+\beta w}\right]^{k} \\
& \leq \frac{W}{d} \sum_{\substack{r=1 \\
u+v=r}}^{\infty} \sum_{w=1}^{\infty} \sum_{k=1}^{\infty}\left[d^{\alpha(u+v)+2 \beta w} 2^{2(u+v)+4 \beta w}|s x|^{\alpha u+\beta w}|s y|^{\alpha v+\beta w}\right]^{k} \\
& =\frac{W}{d} \sum_{\substack{r=1 \\
u+v=r}}^{\infty} \sum_{w=1}^{\infty} \sum_{k=1}^{\infty}\left[4^{\alpha(u+v)+2 \beta w}|d s x|^{\alpha u+\beta w}|d s y|^{\alpha v+\beta w}\right]^{k} \\
& =\frac{W}{d} \sum_{\substack{r=1 \\
u+v=r}}^{\infty} \sum_{w=1}^{\infty} \sum_{k=1}^{\infty}\left[|4 d s x|^{\alpha u+\beta w}|4 d s y|^{\alpha v+\beta w}\right]^{k} \\
& =\frac{W}{d} \sum_{\substack{r=1 \\
u+v=r}}^{\infty} \sum_{w=1}^{\infty} \frac{|4 d s x|^{\alpha u+\beta w}|4 d s y|^{\alpha v+\beta w}}{1-|4 d s x|^{\alpha u+\beta w}|4 d s y|^{\alpha v+\beta w}}
\end{aligned}
$$


where in the penultimate line we assumed that $|4 d s x|<\rho_{1}<\rho<1$ and that $|4 d s y|<\rho_{2}<$ $\rho<1$. When working over these refined polydiscs, the previous inequity becomes

$$
\begin{aligned}
& \left|\sum_{\substack{p=1 \\
m+n=p}}^{\infty} \sum_{k=1}^{\infty}\left(H_{m, n} x^{m} y^{n}\right)^{k}\right| \leq \frac{W}{d} \sum_{\substack{r=1 \\
u+v=r}}^{\infty} \sum_{w=1}^{\infty} \frac{|4 d s x|^{\alpha u+\beta w}|4 d s y|^{\alpha v+\beta w}}{1-\rho^{2}} \\
& \leq \frac{W}{d(1-\rho)} \sum_{\substack{r=1 \\
u+v=r}}^{\infty} \sum_{w=1}^{\infty}|4 d s x|^{\alpha u+\beta w}|4 d s y|^{\alpha v+\beta w} \\
& \leq \frac{W}{d(1-\rho)} \sum_{\substack{r=1 \\
u+v=r}}^{\infty} \sum_{\substack{w=1 \\
\infty}}^{\infty} \rho^{\alpha(u+v)+2 \beta w} \\
& \leq \frac{W}{d(1-\rho)} \sum_{\substack{r=1 \\
u+v=r}}^{\infty} \rho^{\alpha(u+v)} \sum_{w=1}^{\infty} \rho^{2 \beta w} \\
& =\frac{\rho^{2 \beta} W}{d(1-\rho)\left(1-\rho^{2 \beta}\right)}\left[\sum_{u=0}^{\infty} \rho^{\alpha u} \sum_{v=0}^{\infty} \rho^{\alpha v}-1\right] \\
& =\frac{\rho^{2 \beta} W}{d(1-\rho)\left(1-\rho^{2 \beta}\right)}\left[\frac{1}{\left(1-\rho^{\alpha}\right)^{2}}-1\right] \\
& =\frac{\rho^{2 \beta}\left[1-\left(1-\rho^{\alpha}\right)^{2}\right] W}{d(1-\rho)\left(1-\rho^{2 \beta}\right)\left(1-\rho^{\alpha}\right)^{2}} .
\end{aligned}
$$

In conclusion, by using a majorant of a majorant and clever use of inversion, we finally showed

$$
\begin{aligned}
\left|\sum_{\substack{p=1 \\
m+n=p}}^{\infty} \sum_{k=1}^{\infty}\left(H_{m, n} x^{m} y^{n}\right)^{k}\right| & \leq \sum_{\substack{r=1 \\
u+v=r}}^{\infty} \sum_{w=1}^{\infty} \sum_{k=1}^{\infty}\left[\tilde{M}_{\alpha u+\beta w, \alpha v+\beta w}\right]^{k}|x|^{(\alpha u+\beta w) k}|y|^{(\alpha v+\beta w) k} \\
& =\frac{\rho^{2 \beta}\left[1-\left(1-\rho^{\alpha}\right)^{2}\right] W}{d(1-\rho)\left(1-\rho^{2 \beta}\right)\left(1-\rho^{\alpha}\right)^{2}}
\end{aligned}
$$

whenever $|4 d s y|<\rho<1$ and $|4 d s y|<\rho<1$.

Therefore, an estimate for domain of absolute convergence of $\operatorname{LTR} \prod_{\substack{q=1 \\ m+n=q}}^{\infty}\left(I+\hat{R}_{m, n} x^{m} y^{n}\right)$ is

$$
\mathbb{D}_{2}=\left\{(x, y) \in \mathbb{C}^{2}:|x|<\frac{1}{4 d s} \text { and }|y|<\frac{1}{4 d s}\right\} .
$$

Since $O \leq\left|H_{m, n}\right| \leq S_{m, n} \leq R_{m, n} \leq \hat{R}_{m, n}$, Definition 4.4.4 implies that $\mathbb{D}_{2}$ is also an estimate for the domain of absolute convergence of $\operatorname{LTR} \prod_{\substack{q=1 \\ m+n=q}}^{\infty}\left(I-H_{m, n} x^{m} y^{n}\right)^{-1}$ and of $\operatorname{LTR} \prod_{\substack{q=1 \\ m+n=q}}^{\infty}(I+$ $\left.S_{m, n} x^{m} y^{n}\right)^{-1}$. We turn to concrete examples where $F(x, y)$ is a scalar function. 
Example 4.6.2. Let $\alpha \geq 0$ and $\theta_{m, n}$ be a sequence of real numbers, where $(m, n) \in \mathbb{N}_{0} \times$ $\mathbb{N}_{0} \backslash\{(0,0)\}$. Let

$$
F(x, y)=1+\sum_{\substack{p=1 \\ m+n=p}}^{\infty} \exp \left(i \theta_{m, n}\right)(m+n)^{\alpha} x^{m} y^{n}=\prod_{\substack{q=1 \\ m+n=q}}^{\infty}\left(1-h_{m, n} x^{m} y^{n}\right)^{-1},
$$

where $h_{m, n} \in \mathbb{C},(m, n) \in \mathbb{N}_{0} \times \mathbb{N}_{0} \backslash\{(0,0)\}$ are scalar coefficients.

Let $A$ be a d by d matrix. Consider the power series $F(A x, A y)$ together with its IMPPE2 expansion

$$
\begin{aligned}
F(A x, A y) & =I+\sum_{\substack{p=1 \\
m+n=p}}^{\infty} \exp \left(i \theta_{m, n}\right)(m+n)^{\alpha} A^{m+n} x^{m} y^{n} \\
& =L T R \prod_{\substack{q=1 \\
m+n=q}}^{\infty}\left(I-h_{m, n} A^{m+n} x^{m} y^{n}\right)^{-1} .
\end{aligned}
$$

Theorem 4.5.1 along with $\sup _{m+n \geq 1}[m+n]^{\frac{\alpha}{m+n}}=3^{\frac{\alpha}{3}}$ implies that the IMPPE2 of Equation (4.135) converges absolutely in the sense of inverse convergence over the domain

$$
\mathbb{D}_{1}=\left\{(x, y) \in \mathbb{C}^{2}: 2\left(3^{\frac{\alpha}{3}}\right) \rho(A)(|x|+|y|)+2\left[\left(3^{\frac{\alpha}{3}}\right) \rho(A)\right]^{2}|x||y|<1\right\},
$$

and in the sense of Definition 4.4.4 in the polydiscs

$$
\mathbb{D}_{2}=\left\{(x, y) \in \mathbb{C}^{2}:|x|<\frac{1}{4\left(3^{\frac{\alpha}{3}}\right) \rho(A)} \text { and }|y|<\frac{1}{4\left(3^{\frac{\alpha}{3}}\right) \rho(A)}\right\} .
$$

Now let $B, C \in M_{d}(\mathbb{C})$ be two commuting diagonalizable matrices and consider the power series $F(B x, C y)$ together with its associated IMPPE2 expansion

$$
\begin{aligned}
F(B x, C y) & =I+\sum_{\substack{p=1 \\
m+n=p}}^{\infty} \exp \left(i \theta_{m, n}\right)(m+n)^{\alpha}[B x]^{m}[C y]^{n} \\
& =L T R \prod_{\substack{q=1 \\
m+n=q}}^{\infty}\left(I-h_{m, n}[B x]^{m}[C y]^{n}\right)^{-1}
\end{aligned}
$$

To determine the domain of convergence for the IMPPE2 of Equation (4.136) in the sense of inverse convergence, we apply Theorem 4.5.2 [(2.)] with $s=3^{\frac{\alpha}{3}}$ to obtain

$$
\mathbb{D}=\left\{(x, y) \in \mathbb{C}^{2}: 2\left(3^{\frac{\alpha}{3}}\right)[\rho(B)|x|+\rho(C)|y|]+2\left(3^{\frac{2 \alpha}{3}}\right) \rho(B) \rho(C)|x||y|<1\right\},
$$


and to obtain the domain of absolute convergence in the sense of Definition 4.4.4, we apply Theorem 4.5.2 [(3.)] to get

$$
\mathbb{D}_{2}=\left\{(x, y) \in \mathbb{C}^{2}:|x|<\frac{1}{4\left(3^{\frac{\alpha}{3}}\right) \rho(B)} \text { and }|y|<\frac{1}{4\left(3^{\frac{\alpha}{3}}\right) \rho(C)}\right\}
$$

Example 4.6.3. Let $p \in \mathbb{N}$ and consider the scalar bivariate exponential function together with its IPPE2 expansion

$$
F(x, y)=\exp (x y)=1+\sum_{p=1}^{\infty} \frac{1}{p !}(x y)^{p}=\prod_{p=1}^{\infty}\left(1-h_{q}(x y)^{q}\right)^{-1},
$$

where $h_{q} \in \mathbb{C}$

Let $A$ be a d by $d$ matrix. Consider the power series $F(A x, A y)$ together with its IMPPE2 expansion

$$
F(A x, A y)=\exp \left(A^{2} x y\right)=I+\sum_{p=1}^{\infty} \frac{A^{2 p}}{p !}(x y)^{p}=L T R \prod_{p=1}^{\infty}\left(I-h_{q} A^{2 q}(x y)^{q}\right)^{-1} .
$$

By the Jordan-Chevalley decomposition [see [30], page 17], every matrix $A \in M_{d}(\mathbb{C})$ can be uniquely decomposed into the sum of a diagonalizable and a nilpotent matrix

$$
A=V+Q
$$

The matrices $V, Q \in M_{d}(\mathbb{C})$ satisfy the following properties:

1) $V$ is diagonalizable and $Q$ nilpotent, i.e.

$$
T^{-1} V T=D=\left(\begin{array}{cccc}
u_{1} & 0 & \cdots & 0 \\
0 & u_{2} & 0 & \vdots \\
\vdots & 0 & \ddots & 0 \\
0 & 0 & \cdots & u_{d}
\end{array}\right), Q^{d}=O
$$

where $u_{j}, j=1,2, \ldots, d$ are the $d$ eigenvalues of A counting multiplicities.

2) $V$ and $Q$ commute, i.e. $V Q=V Q$,

3) $V$ and $Q$ are polynomials in $A$, i.e. $\exists p(x)$ s.t $V=p(A)$ and $Q=A-p(A)$. 
We will use this Jordan-Chevalley decomposition to rewrite $\exp (2 A)$ as

$$
\begin{aligned}
& \exp \left(A^{2}\right)=\prod_{p=1}^{\infty}\left(I-h_{q} A^{2 q}\right)^{-1} \\
= & \exp [(V+Q)(V+Q)]=\exp \left[V^{2}+2 Q V+Q^{2}\right]=\left[\exp \left(V^{2}\right)\right][\exp (2 Q V)]\left[\exp \left(Q^{2}\right)\right] \\
= & {\left[\prod_{q=1}^{\infty}\left(I-h_{q} V^{2 q}\right)^{-1}\right]\left[\prod_{q=1}^{d}\left(I-h_{q}[2 Q V]^{2 q}\right)^{-1}\right]\left[\prod_{q=1}^{d}\left(I-h_{q} Q^{2 q}\right)^{-1}\right] . }
\end{aligned}
$$

To determine the domain of convergence for $\exp \left(A^{2}\right)=\prod_{q=1}^{\infty}\left(I-h_{q} A^{2 q}\right)^{-1}$, it suffices to determine a domain of convergence for $\prod_{q=1}^{\infty}\left(I-h_{q} V^{2 q}\right)^{-1}$. Since $\sup \left(\frac{1}{p !}\right)^{\frac{1}{p}}=1$, Theorem 4.5.1[(2.)] implies an estimate for the domain of convergence in the sense of inverse convergence of $\prod_{q=1}^{\infty}\left(I-h_{q} V^{2 q}\right)^{-1}$ is

$$
\mathbb{D}_{1}=\left\{(x, y) \in \mathbb{C}^{2}: 4 \rho(V)+2[\rho(V)]^{2}<1\right\} .
$$

We may use this information to obtain an upper bound on the spectrum of $V$. Since $2[\rho(V)]^{2}<1$, we deduce that

$$
\rho(V)<\frac{1}{\sqrt{2}}
$$

A similar calculation shows that

$$
\rho(A)<\frac{1}{\sqrt{2}}
$$

and to obtain the domain of absolute convergence in the sense of Definition 4.4.4, we apply Theorem 4.5.2 [(3.)] to get

$$
\mathbb{D}_{2}=\left\{(x, y) \in \mathbb{C}^{2}:|x|<\frac{1}{4 \rho(A)} \text { and }|y|<\frac{1}{4 \rho(A)}\right\} .
$$

Now let $B, C \in M_{d}(\mathbb{C})$ be two commuting diagonalizable matrices. Consider the power series $F(B x, C y)$ together with its IMPPE2 expansion

$$
F(B x, C y)=\exp (B C x y)=I+\sum_{p=1}^{\infty} \frac{(B C)^{p}}{p !}(x y)^{p}=L T R \prod_{q=1}^{\infty}\left(I-h_{q}(B C x y)^{q}\right)^{-1} .
$$

To determine the domain of convergence for $\exp (B C)=\prod_{q=1}^{\infty}\left(I-h_{q}(B C)^{q}\right)^{-1}$, we use Theorem 4.5.2[(2.)], along with $\sup \left(\frac{1}{p !}\right)^{\frac{1}{p}}=1$, to obtain the following estimate in the sense of inverse convergence:

$$
\mathbb{D}_{1}^{\prime}=\left\{(x, y) \in \mathbb{C}^{2}: 2[\rho(B)+\rho(C)]+2 \rho(B) \rho(C)<1\right\} .
$$


We may use this information to obtain an upper bound on the spectrum of $B$ and $C$. Since $2 \rho(B) \rho(C)<1$, we deduce that

$$
\rho(B), \rho(C)<\frac{1}{2},
$$

and to obtain the domain of absolute convergence in the sense of Definition 4.4.4, we apply Theorem 4.5.2 [(3.)] to get

$$
\mathbb{D}_{2}^{\prime}=\left\{(x, y) \in \mathbb{C}^{2}:|x|<\frac{1}{4 \rho(B)} \text { and }|y|<\frac{1}{4 \rho(C)}\right\} .
$$

Example 4.6.4. Let $A, B, C, \in M_{d}(\mathbb{C})$, where $B$ and $C$ are diagonalizable with $B C=C B \neq$ $O$. The techniques of Example 4.6.3 may also be applied to other matrix function such as

$$
\begin{aligned}
& I+\log \left(I-A^{2}\right), \quad A^{-2} \log \left(I-A^{2}\right), \quad \cos A^{2}, \quad I+\sin A^{2}, \quad A^{2} \sin A^{2}, \\
& \cosh A^{2}, \quad I+\sinh A^{2}, \quad A^{-2} \tan A^{2}, \quad \arccos A^{2}, \quad I+\arcsin A^{2}, \\
& I+\log (I-B C), \quad B C \log (I-B C), \quad \cos B C, \quad I+\sin B C, \quad B C \sin B C, \\
& \cosh B C, \quad I+\sinh B C, \quad B C \tan B C, \quad \arccos B C, \quad I+\arcsin B C .
\end{aligned}
$$

We will demonstrate these techniques for $\cos A^{2}, \cos B C, A^{2} \sin A^{-2}$, and $[B C]^{-1} \sin B C$, leaving the rest to the reader. Let $h_{q}$ and $\hat{h}_{q}$ be the scalar coefficients in the IPPE2 expansions of the even scalar functions

$$
\begin{aligned}
& \cos x y=1+\sum_{p=1}^{\infty} \frac{(-1)^{p} x^{2 p} y^{2 p}}{(2 p) !}=\prod_{q=1}^{\infty}\left(1-h_{q} x^{2 q} y^{2 q}\right)^{-1} \\
& \sin x y=x y\left[1+\sum_{p=1}^{\infty} \frac{(-1)^{p} x^{2 p} y^{2 p}}{(2 p+1) !}\right]=x y \prod_{q=1}^{\infty}\left(1-\hat{h}_{q} x^{2 q} y^{2 q}\right)^{-1} .
\end{aligned}
$$

First,

$$
\cos A^{2}=I+\sum_{p=1}^{\infty} \frac{(-1)^{p} A^{4 p}}{(2 p) !}=\prod_{q=1}^{\infty}\left(I-h_{q} A^{4 q}\right)^{-1} .
$$

Since $s:=\sup _{p \geq 1}\left|\frac{(-1)^{p}}{(2 p) !}\right|^{\frac{1}{p}}=\frac{1}{2}$, Theorem 4.5.1[(2.)] implies an estimate for domain of convergence of $\prod_{q=1}^{\infty}\left(I-h_{q} A^{4 q}\right)^{-1}$ in the sense of inverse convergence is

$$
\mathbb{D}_{1}=\left\{(x, y) \in \mathbb{C}^{2}: 2 \rho(A)+\frac{1}{2}[\rho(A)]^{2}<1\right\} .
$$


We may use this information to obtain an upper bound on the spectrum of A. Since $\frac{1}{2}[\rho(A)]^{2}<1$, we deduce that

$$
\rho(A)<\sqrt{2}
$$

and to obtain the domain of absolute convergence in the sense of Definition 4.4.4, we apply Theorem 4.5.2 [(3.)] to get

$$
\mathbb{D}_{2}=\left\{(x, y) \in \mathbb{C}^{2}:|x|<\frac{1}{2 \rho(A)} \text { and }|y|<\frac{1}{2 \rho(A)}\right\} .
$$

For

$$
\cos B C=I+\sum_{p=1}^{\infty} \frac{(-1)^{p} B^{2 p} C^{2 p}}{(2 p) !}=\prod_{q=1}^{\infty}\left(I-h_{q} B^{2 q} C^{2 q}\right)^{-1} .
$$

Theorem 4.5.2[(2.)] implies an estimate for the domain of convergence of $\prod_{q=1}^{\infty}\left(1-h_{q} x^{2 q} y^{2 q}\right)^{-1}$ in the sense of inverse convergence is

$$
\mathbb{D}_{1}^{\prime}=\left\{(x, y) \in \mathbb{C}^{2}:[\rho(B)+\rho(C)]+\frac{1}{2} \rho(B) \rho(C)<1\right\} .
$$

We may use this information to obtain an upper bound on the spectrum of $B$ and $C$. Since $\frac{1}{2} \rho(B) \rho(C)<1$, we deduce that

$$
\rho(B), \rho(C)<2,
$$

and to obtain the domain of absolute convergence in the sense of Definition 4.4.4, we apply Theorem 4.5.2 [(3.)] to get

$$
\mathbb{D}_{2}^{\prime}=\left\{(x, y) \in \mathbb{C}^{2}:|x|<\frac{1}{2 \rho(B)} \text { and }|y|<\frac{1}{2 \rho(C)}\right\} .
$$

Next

$$
\sin A^{2}=A^{2}\left[I+\sum_{p=1}^{\infty} \frac{(-1)^{p} A^{4 p}}{(2 p+1) !}\right]=A^{2} \prod_{q=1}^{\infty}\left(I-\hat{h}_{q} A^{4 q}\right)^{-1}
$$

Since $s:=\sup _{p \geq 1}\left|\frac{(-1)^{p}}{(2 p+1) !}\right|^{\frac{1}{p}}=\frac{1}{6}$, Theorem 4.5.1[(2.)] implies an estimate for domain of convergence of $\prod_{q=1}^{\infty}\left(I-\hat{h}_{q} A^{4 q}\right)^{-1}$ in the sense of inverse convergence is

$$
\mathbb{D}_{1}=\left\{(x, y) \in \mathbb{C}^{2}: \frac{2}{3} \rho(A)+\frac{1}{18}[\rho(A)]^{2}<1\right\} .
$$


We may use this information to obtain an upper bound on the spectrum of A. Since $\frac{1}{18}[\rho(A)]^{2}<1$, we deduce that

$$
\rho(A)<3 \sqrt{2},
$$

and to obtain the domain of absolute convergence in the sense of Definition 4.4.4, we apply Theorem 4.5.2 [(3.)] to get

$$
\mathbb{D}_{2}=\left\{(x, y) \in \mathbb{C}^{2}:|x|<\frac{3}{2 \rho(A)} \text { and }|y|<\frac{3}{2 \rho(A)}\right\} .
$$

Finally it can be shown that an estimate for the domain of convergence of

$$
\sin B C=B C\left[I+\sum_{p=1}^{\infty} \frac{(-1)^{p} B^{2 p} C^{2 p}}{(2 p+1) !}\right]=B C \prod_{q=1}^{\infty}\left(I-\hat{h}_{q} B^{2 p} C^{2 p}\right)^{-1},
$$

in the sense of inverse convergence is

$$
\mathbb{D}_{1}^{\prime}=\left\{(x, y) \in \mathbb{C}^{2}: \frac{1}{3}[\rho(B)+\rho(C)]+\frac{1}{18} \rho(B) \rho(C)<1\right\},
$$

and that

$$
\rho(B), \rho(C)<18,
$$

and to obtain the domain of absolute convergence in the sense of Definition 4.4.4, we apply Theorem 4.5.2 [(3.)] to get

$$
\mathbb{D}_{2}=\left\{(x, y) \in \mathbb{C}^{2}:|x|<\frac{3}{2 \rho(B)} \text { and }|y|<\frac{3}{2 \rho(C)}\right\} .
$$

Example 4.6.5. Consider the scalar bivariate exponential function together with its IPPE2 expansion

$$
F(x, y)=\exp (x+y)=1+\sum_{\substack{p=1 \\ m+n=p}}^{\infty} \frac{1}{m ! n !} x^{m} y^{n}=\prod_{\substack{q=1 \\ m+n=q}}^{\infty}\left(1-h_{m, n} x^{m} y^{n}\right)^{-1},
$$

where $h_{m, n} \in \mathbb{C}$. By the Jordan-Chevalley decomposition, every matrix $A \in M_{d}(\mathbb{C})$ can be uniquely decomposed into the sum of a diagonalizable and a nilpotent matrix

$$
A=V+Q
$$

The matrices $V, Q \in M_{d}(\mathbb{C})$ satisfy the following properties: 
1) $V$ is diagonalizable and $Q$ nilpotent, i.e.

$$
T^{-1} V T=D=\left(\begin{array}{cccc}
u_{1} & 0 & \cdots & 0 \\
0 & u_{2} & 0 & \vdots \\
\vdots & 0 & \ddots & 0 \\
0 & 0 & \cdots & u_{d}
\end{array}\right), Q^{d}=O
$$

where $u_{j}, j=1,2, \ldots, d$ are the $d$ eigenvalues of $A$ counting multiplicities.

2) $V$ and $Q$ commute, i.e. $V Q=V Q$,

3) $V$ and $Q$ are polynomials in $A$, i.e. $\exists p(x)$ s.t $V=p(A)$ and $Q=A-p(A)$.

We will use this Jordan-Chevalley decomposition to rewrite $\exp (2 A)$ as

$$
\begin{aligned}
\exp (2 A) & =\prod_{\substack{q=1 \\
m+n=q}}^{\infty}\left(I-h_{m, n} A^{m+n}\right)^{-1} \\
& =\exp (2 V+2 Q)=\exp (2 V) \exp (2 Q) \\
& =\left[\prod_{\substack{q=1 \\
m+n=q}}^{\infty}\left(I-h_{m, n} V^{m+n}\right)^{-1}\right]\left[\prod_{\substack{q=1 \\
m+n=q}}^{d}\left(I-h_{m, n} Q^{m+n}\right)^{-1}\right] .
\end{aligned}
$$

To determine the domain of convergence for $\exp (2 A)=\prod_{\substack{q=1 \\ m+n=q}}^{\infty}\left(I-h_{m, n} A^{m+n}\right)^{-1}$, it suffices to determine a domain of convergence for $\prod_{\substack{q=1 \\ m+n=q}}^{\infty}\left(I-h_{m, n} V^{m+n}\right)^{-1}$. Since $\sup _{m+n \geq 1}\left[\frac{1}{m ! n !}\right]^{\frac{1}{m+n}}=$ 1, Theorem 4.5.1[(2.)] implies an estimate for the domain of convergence of $\prod_{\substack{q=1 \\ m+n=q}}^{\infty}(I-$ $\left.h_{m, n} V^{m+n}\right)^{-1}$ in the sense of inverse convergence is

$$
\mathbb{D}_{1}=\left\{(x, y) \in \mathbb{C}^{2}: 4 \rho(V)+2[\rho(V)]^{2}<1\right\} .
$$

We may use this information to obtain an upper bound on the spectrum of $V$. Since $2[\rho(V)]^{2}<1$, we deduce that

$$
\rho(V)<\frac{1}{\sqrt{2}}
$$

A similar calculation shows that

$$
\rho(A)<\frac{1}{\sqrt{2}}
$$


and to obtain the domain of absolute convergence in the sense of Definition 4.4.4, we apply Theorem 4.5.2 [(3.)] to get

$$
\mathbb{D}_{2}=\left\{(x, y) \in \mathbb{C}^{2}:|x|<\frac{1}{4 \rho(A)} \text { and }|y|<\frac{1}{4 \rho(A)}\right\} .
$$

Now let $B, C \in M_{d}(\mathbb{C})$ be two commuting diagonalizable matrices. Consider the power series $F(B x, C y)$ together with its IMPPE2 expansion

$$
\begin{aligned}
F(B x, C y)=\exp (B x+C y) & =I+\sum_{\substack{p=1 \\
m+n=p}}^{\infty} \frac{B^{m} C^{n}}{m ! n !} x^{m} y^{n} \\
& =L T R \prod_{\substack{q=1 \\
m+n=q}}^{\infty}\left(I-h_{m, n} B^{m} C^{n} x^{m} y^{n}\right)^{-1} .
\end{aligned}
$$

To determine the domain of convergence for $\exp (B+C)=\prod_{\substack{q=1 \\ m+n=q}}^{\infty}\left(I-h_{m, n} B^{m} C^{n}\right)^{-1}$, we use Theorem 4.5.2, along with $\sup _{m+n \geq 1}\left[\frac{1}{m ! n !}\right]^{\frac{1}{m+n}}=1$, to obtain the following estimate in the sense of inverse convergence.

$$
\mathbb{D}_{1}^{\prime}=\left\{(x, y) \in \mathbb{C}^{2}: 2[\rho(B)+\rho(C)]+2 \rho(B) \rho(C)<1\right\} .
$$

We may use this information to obtain an upper bound on the spectrum of $B$ and $C$. Since $2 \rho(B) \rho(C)<1$, we deduce that

$$
\rho(B), \rho(C)<\frac{1}{2},
$$

and to obtain the domain of absolute convergence in the sense of Definition 4.4.4, we apply Theorem 4.5.2 [(3.)] to get

$$
\mathbb{D}_{2}^{\prime}=\left\{(x, y) \in \mathbb{C}^{2}:|x|<\frac{1}{4 \rho(B)} \text { and }|y|<\frac{1}{4 \rho(C)}\right\} .
$$

Example 4.6.6. Let $A, B, C, \in M_{d}(\mathbb{C})$, where $B$ and $C$ are diagonalizable with $B C=C B \neq$ $O$. The techniques of Example 4.6.5 may also be applied to other matrix function such as $I+\log (I-2 A), \quad(2 A)^{-1} \log (I-2 A), \quad \cos 2 A, \quad I+\sin 2 A, \quad 2 A \sin 2 A$, $\cosh 2 A, \quad I+\sinh 2 A, \quad(2 A)^{-1} \tan 2 A, \quad \arccos 2 A, \quad I+\arcsin 2 A$, $I+\log (I-[B+C]), \quad[B+C] \log (I-[B+C]), \quad \cos B+C, \quad I+\sin (B+C)$, $[B+C] \sin (B+C), \quad \cosh (B+C), \quad I+\sinh [B+C], \quad[B+C] \tan (B+C)$, $\arccos (B+C), \quad I+\arcsin (B+C)$. 
We will demonstrate these techniques for $\cos 2 A, \cos (B+C),[2 A]^{-1} \sin 2 A$, and $[B+$ $C]^{-1} \sin (B+C)$, leaving the rest to the reader. Let $h_{m, n}$ and $\hat{h}_{m, n}$ be the scalar coefficients in the IPPE2 expansions of the even scalar functions

$$
\begin{aligned}
\cos (x+y) & =1+\sum_{\substack{p=1 \\
m+n=2 p}}^{\infty} \frac{(-1)^{\frac{m+n}{2}}}{m ! n !} x^{m} y^{n}=\prod_{\substack{q=1 \\
m+n=2 q}}^{\infty}\left(1-h_{m, n} x^{m} y^{n}\right)^{-1} \\
\sin (x+y) & =(x+y)\left[1+(x+y)^{-1} \sum_{n=1}^{\infty} \frac{(-1)^{n}}{(2 n+1) !}(x+y)^{2 n+1}\right] \\
& =(x+y)\left[1+\sum_{n=1}^{\infty} \frac{(-1)^{n}}{(2 n+1) !} \sum_{j=0}^{2 n}\left(\begin{array}{c}
2 n \\
j
\end{array}\right) x^{j} y^{2 n-j}\right] \\
& =(x+y)\left[1+\sum_{\substack{p=1 \\
m+n=2 p}}^{\infty} \frac{(-1)^{\frac{m+n}{2}}}{(m+n+1) !}\left(\begin{array}{c}
m+n \\
m
\end{array}\right) x^{m} y^{n}\right] \\
& =(x+y) \prod_{\substack{q=1 \\
m+n=2 q}}^{\infty}\left(1-\hat{h}_{m, n} x^{m} y^{n}\right)^{-1}
\end{aligned}
$$

First,

$$
\cos 2 A=I+\sum_{\substack{p=1 \\ m+n=2 p}}^{\infty} \frac{(-1)^{\frac{m+n}{2}}}{m ! n !} A^{m+n}=\prod_{\substack{q=1 \\ m+n=2 q}}^{\infty}\left(I-h_{m, n} A^{m+n}\right)^{-1}
$$

Since $s:=\sup _{\substack{p \geq 1 \\ m+n=2 p}}\left|\frac{(-1)^{\frac{m+n}{2}}}{m ! n !}\right|^{\frac{1}{m+n}}=1$, Theorem 4.5.1 implies an estimate for domain of convergence of $\prod_{\substack{q=1 \\ m+n=2 q}}^{\infty}\left(I-h_{m, n} A^{m+n}\right)^{-1}$ in the sense of inverse convergence is

$$
\mathbb{D}_{1}=\left\{(x, y) \in \mathbb{C}^{2}: 4 \rho(A)+2[\rho(A)]^{2}<1\right\} .
$$

We may use this information to obtain an upper bound on the spectrum of A. Since $2[\rho(A)]^{2}<1$, we deduce that

$$
\rho(A)<\frac{1}{\sqrt{2}},
$$

and to obtain the domain of absolute convergence in the sense of Definition 4.4.4, we apply Theorem 4.5.2 [(3.)] to get

$$
\mathbb{D}_{2}=\left\{(x, y) \in \mathbb{C}^{2}:|x|<\frac{1}{4 \rho(A)} \text { and }|y|<\frac{1}{4 \rho(A)}\right\} .
$$


For

$$
\cos (B+C)=I+\sum_{\substack{p=1 \\ m+n=2 p}}^{\infty} \frac{(-1)^{\frac{m+n}{2}}}{m ! n !} B^{m} C^{n}=\prod_{\substack{q=1 \\ m+n=2 q}}^{\infty}\left(I-h_{m, n} B^{m} C^{n}\right)^{-1}
$$

Theorem 4.5.2 [(2.)] implies an estimate for the domain of convergence of $\prod_{\substack{q=1 \\ m+n=2 q}}^{\infty}(I-$ $\left.h_{m, n} B^{m} C^{n}\right)^{-1}$ in the sense of inverse convergence is

$$
\mathbb{D}_{1}^{\prime}=\left\{(x, y) \in \mathbb{C}^{2}: 2[\rho(B)+\rho(C)]+2 \rho(B) \rho(C)<1\right\} .
$$

We may use this information to obtain an upper bound on the spectrum of $B$ and $C$. Since $2 \rho(B) \rho(C)<1$, we deduce that

$$
\rho(B), \rho(C)<\frac{1}{2} .
$$

and to obtain the domain of absolute convergence in the sense of Definition 4.4.4, we apply Theorem 4.5.2 [(3.)] to get

$$
\mathbb{D}_{2}^{\prime}=\left\{(x, y) \in \mathbb{C}^{2}:|x|<\frac{1}{4 \rho(B)} \text { and }|y|<\frac{1}{4 \rho(C)}\right\} .
$$

Next

$$
\sin 2 A=2 A\left[I+\sum_{\substack{p=1 \\
m+n=2 p}}^{\infty} \frac{(-1)^{\frac{m+n}{2}}}{(m+n+1) !}\left(\begin{array}{c}
m+n \\
m
\end{array}\right) A^{m+n}\right]=2 A \prod_{\substack{q=1 \\
m+n=2 q}}^{\infty}\left(I-\hat{h}_{m, n} A^{m+n}\right)^{-1} .
$$

Since $s:=\sup _{\substack{p=1 \\ m+n=2 p}}\left|\frac{(-1)^{\frac{m+n}{2}}}{(m+n+1) !}\left(\begin{array}{c}m+n \\ m\end{array}\right)\right|^{\frac{1}{m+n}}=\frac{1}{\sqrt{3}}$, Theorem 4.5.1 implies an estimate for domain of convergence of $\prod_{\substack{q=1 \\ m+n=2 q}}^{\infty}\left(I-\hat{h}_{m, n} A^{m+n}\right)^{-1}$ in the sense of inverse convergence is

$$
\mathbb{D}_{1}=\left\{(x, y) \in \mathbb{C}^{2}: \frac{4}{\sqrt{3}} \rho(A)+\frac{2}{3}[\rho(A)]^{2}<1\right\} .
$$

We may use this information to obtain an upper bound on the spectrum of A. Since $\frac{2}{3}[\rho(A)]^{2}<1$, we deduce that

$$
\rho(A)<\frac{\sqrt{3}}{\sqrt{2}} .
$$


and to obtain the domain of absolute convergence in the sense of Definition 4.4.4, we apply Theorem 4.5.2 [(3.)] to get

$$
\mathbb{D}_{2}=\left\{(x, y) \in \mathbb{C}^{2}:|x|<\frac{\sqrt{3}}{4 \rho(A)} \text { and }|y|<\frac{\sqrt{3}}{4 \rho(A)}\right\} .
$$

Finally it can be shown that an estimate for the domain of convergence of

$$
\begin{aligned}
\sin (B+C) & =[B+C]\left[I+\sum_{\substack{p=1 \\
m+n=2 p}}^{\infty} \frac{(-1)^{\frac{m+n}{2}}}{(m+n+1) !}\left(\begin{array}{c}
m+n \\
m
\end{array}\right) B^{m} C^{n}\right] \\
& =[B+C] \prod_{\substack{q=1 \\
m+n=2 q}}^{\infty}\left(I-\hat{h}_{m, n} B^{m} C^{n}\right)^{-1},
\end{aligned}
$$

in the sense of inverse convergence is

$$
\mathbb{D}_{1}^{\prime}=\left\{(x, y) \in \mathbb{C}^{2}: \frac{2}{\sqrt{3}}[\rho(B)+\rho(C)]+\frac{2}{3} \rho(B) \rho(C)<1\right\},
$$

and that

$$
\rho(B), \rho(C)<\frac{3}{2},
$$

and to obtain the domain of absolute convergence in the sense of Definition 4.4.4, we apply Theorem 4.5.2 [(3.)] to get

$$
\mathbb{D}_{2}^{\prime}=\left\{(x, y) \in \mathbb{C}^{2}:|x|<\frac{\sqrt{3}}{4 \rho(B)} \text { and }|y|<\frac{\sqrt{3}}{4 \rho(C)}\right\} .
$$




\section{Appendix A}

\section{Double Series}

In order to study the factorization of complex bivariate analytic function, we will need to know some things about double series. As in the case of single series $\sum_{n} a_{n}$ we will define convergence of a double series $\sum_{m, n=0} a_{m, n}$ in terms of convergence of double sequence $\left(a_{m, n}\right)_{m, n=0}^{\infty}$.

\section{A.1 Double sequence}

This section on double sequences is meant to be used as a preparation for the upcoming sections on double series.

A double sequence (in $\mathbb{C}$ ) is a complex-valued function $f$ whose domain of definition is the set $\mathbb{N}_{0}^{2}=\mathbb{N}_{0} \times \mathbb{N}_{0}:=\left\{(m, n): m, n \in \mathbb{N}_{0}\right\}$ and whose domain contained in $\mathbb{C}$, where $\mathbb{N}_{0}=\mathbb{N} \cup\{0\}$ and $a_{m, n}=f(m, n)$ for all $(m, n) \in \mathbb{N}_{0}^{2}$. We will denote double sequences by $\left(a_{m, n}\right)_{(m, n) \in \mathbb{N}_{0}^{2}}$. The double sequence may also be written schematically as follows:

$$
\left(a_{m, n}\right)=\begin{array}{ccccc}
a_{0,0} & a_{0,1} & \ldots & a_{0, n} & \ldots \\
a_{1,0} & a_{1,1} & \ldots & a_{1, n} & \ldots \\
\vdots & \vdots & \vdots & \vdots & \\
a_{m, 0} & a_{m, 1} & \ldots & a_{m, n} & \ldots \\
\vdots & \vdots & \vdots & \vdots & \ddots
\end{array}
$$

Most of the results about a double sequence are analogues to results about a single sequence.

Definition A.1.1. [28]. We say that a complex double sequence $\left(a_{m, n}\right)$ is convergent if there exists $a \in \mathbb{C}$ satisfying the following condition: For every $\varepsilon>0$, there exists 
$\left(m_{0}, n_{0}\right) \in \mathbb{N}_{0}^{2}$ such that

$$
\left|a_{m, n}-a\right|<\varepsilon \quad \forall \quad(m, n) \succeq\left(m_{0}, n_{0}\right)
$$

A double sequence that is not convergent is said to be divergent. In particular, if for every $\alpha \in \mathbb{R}^{+}$, there is $\left(m_{0}, n_{0}\right) \in \mathbb{N}_{0}^{2}$ such that $\left|a_{m, n}\right|>\alpha$ for all $(m, n) \succeq\left(m_{0}, n_{0}\right)$, then we say that $\left(a_{m, n}\right)$ diverges to $\infty$ and we write $a_{m, n} \rightarrow \infty$. Similarly, $\left(a_{m, n}\right)$ diverges to $-\infty$ if for every $\beta \in \mathbb{R}^{+}$, there is $\left(m_{0}, n_{0}\right) \in \mathbb{N}_{0}^{2}$ such that $a_{m, n}<-\beta$ for all $(m, n) \succeq\left(m_{0}, n_{0}\right)$. For example, if $\left(a_{m, n}\right):=1 /(m+n)$ if $(m, n) \in \mathbb{N}_{0}^{2} \backslash\{(0,0)\}$ and $\left(a_{m, n}\right):=0$ if $(m, n)=(0,0)$, $b_{m, n}:=m+n$, and $c_{m, n}=(i)^{m+n}$ for $(m, n) \in \mathbb{N}_{0}^{2}$, then $a_{m, n} \rightarrow 0$ and $\left(b_{m, n}\right)$ diverges to $\infty$, while the double sequence $\left(c_{m, n}\right)$ is bounded, but divergent.

Theorem A.1.1. Suppose $a_{m, n}=x_{m, n}+i y_{m, n}$ and $a=x+i y$. Then,

$$
\lim _{(m, n) \rightarrow(\infty, \infty)} a_{m, n}=a
$$

if and only if

$$
\lim _{(m, n) \rightarrow(\infty, \infty)} x_{m, n}=x \quad \text { and } \quad \lim _{(m, n) \rightarrow(\infty, \infty)} y_{m, n}=y
$$

Proof. First, we suppose the condition (A.1) holds. Then, for each positive number $\varepsilon$ there is $\left(m_{0}, n_{0}\right) \in \mathbb{N}_{0}^{2}$ such that

$$
\left|\left(x_{m, n}+i y_{m, n}\right)-(x+i y)\right|<\varepsilon, \quad \text { whenever } \quad(m, n) \succeq\left(m_{0}, n_{0}\right) .
$$

Since,

$$
\left|x_{m, n}-x\right| \leq\left|\left(x_{m, n}-x\right)+i\left(y_{m, n}-y\right)\right|=\left|\left(x_{m, n}+i y_{m, n}\right)-(x+i y)\right|,
$$

and

$$
\left|y_{m, n}-y\right| \leq\left|\left(x_{m, n}-x\right)+i\left(y_{m, n}-y\right)\right|=\left|\left(x_{m, n}+i y_{m, n}\right)-(x+i y)\right|,
$$

consequently, we have

$$
\left|x_{m, n}-x\right|<\varepsilon \quad \text { and } \quad\left|y_{m, n}-y\right|<\varepsilon, \quad \text { whenever } \quad(m, n) \succeq\left(m_{0}, n_{0}\right) .
$$

Evidently, conditions (A.2) are satisfied.

Conversely, assume that conditions (A.2) hold. Then, there exist for each positive $\varepsilon$, $\left(m_{1}, n_{1}\right),\left(m_{2}, n_{2}\right) \in \mathbb{N}_{0}^{2}$ such that

$$
\left|x_{m, n}-x\right|<\frac{\varepsilon}{2}, \quad \text { whenever } \quad(m, n) \succeq\left(m_{1}, n_{1}\right),
$$


and

$$
\left|y_{m, n}-y\right|<\frac{\varepsilon}{2}, \quad \text { whenever } \quad(m, n) \succeq\left(m_{2}, n_{2}\right) .
$$

Let $\left(m_{0}, n_{0}\right)=\max \left\{\left(m_{1}, n_{1}\right),\left(m_{2}, n_{2}\right)\right\}$. Then, we have

$$
\left|x_{m, n}-x\right|<\frac{\varepsilon}{2}, \quad \text { and } \quad\left|y_{m, n}-y\right|<\frac{\varepsilon}{2}, \quad \text { whenever } \quad(m, n) \succeq\left(m_{0}, n_{0}\right) \text {. }
$$

Since,

$$
\begin{aligned}
\left|\left(x_{m, n}+i y_{m, n}\right)-(x+i y)\right| & =\left|\left(x_{m, n}-x\right)+i\left(y_{m, n}-y\right)\right| \\
& \leq\left|x_{m, n}-x\right|+\left|y_{m, n}-y\right| .
\end{aligned}
$$

Hence,

$$
\left|a_{m, n}-a\right| \leq \frac{\varepsilon}{2}+\frac{\varepsilon}{2}=\varepsilon, \quad \text { whenever } \quad(m, n) \succeq\left(m_{0}, n_{0}\right) .
$$

Thus, the condition A.2 holds.

The Limit Theorem for double sequences says that if $a_{m, n} \rightarrow a$ and $b_{m, n} \rightarrow b$, then $a_{m, n}+b_{m, n} \rightarrow a+b, r a_{m, n} \rightarrow r a$ for any $r \in \mathbb{C}, a_{m, n} b_{m, n} \rightarrow a b$, and if $a \neq 0$, then there is $\left(m_{0}, n_{0}\right) \in \mathbb{N}_{0}^{2}$ such that $a_{m, n} \neq 0$ for all $(m, n) \succeq\left(m_{0}, n_{0}\right)$ and $1 / a_{m, n} \rightarrow 1 / a$; further, if there is $\left(m_{1}, n_{1}\right) \in \mathbb{N}_{0}^{2}$ such that $a_{m, n} \leq b_{m, n}$ for all $(m, n) \succeq\left(m_{1}, n_{1}\right)$, then $a \leq b$, and if $a_{m, n} \geq 0$ for all $(m, n) \in \mathbb{N}_{0}^{2}$, then $a_{m, n}^{1 / k} \rightarrow a^{1 / k}$ for any $k \in \mathbb{N}$. Also, if $a_{m, n} \rightarrow a$, then $\left|a_{m, n}\right| \rightarrow|a|$, but the converse does not hold unless $a=0$.

Another useful result is the Sandwich Theorem for real double sequences: If $\left(a_{m, n}\right)$, $\left(b_{m, n}\right)$, and $\left(c_{m, n}\right)$ are real double sequences such that $a_{m, n} \leq c_{m, n} \leq b_{m, n}$, and if $c \in \mathbb{C}$ is such that $a_{m, n} \rightarrow c$ and $b_{m, n} \rightarrow c$, then $c_{m, n} \rightarrow c$ as well.

A double sequence $\left(a_{m, n}\right)$ is called a Cauchy double sequence if for every $\varepsilon>0$, there exists $\left(m_{0}, n_{0}\right) \in \mathbb{N}_{0}^{2}$ such that

$$
\left|a_{p, q}-a_{m, n}\right|<\varepsilon \quad \text { for all }(m, n),(p, q) \succeq\left(m_{0}, n_{0}\right) .
$$

The following result enables us to confirm that a double sequence converges without specifying its limit.

Theorem A.1.2. (Cauchy Criterion for Double Sequences)[28]. A double sequence is convergent if and only if it is a Cauchy double sequence.

Proof. Let $a_{m, n} \rightarrow a$ as $(m, n) \rightarrow(\infty, \infty)$ and let $\varepsilon>0$. Then there exists $\left(m_{0}, n_{0}\right) \in \mathbb{N}_{0}^{2}$ such that $\left|a_{m, n}-a\right|<\varepsilon$ for all $(m, n) \succeq\left(m_{0}, n_{0}\right)$. Hence, 
for all $(p, q) \succeq(m, n) \succeq\left(m_{0}, n_{0}\right)$, we have

$$
\begin{aligned}
\left|a_{p, q}-a_{m, n}\right| & =\left|a_{p, q}-a+a-a_{m, n}\right| \\
& \leq\left|a_{p, q}-a\right|+\left|a_{m, n}-a\right| \\
& \leq \frac{\varepsilon}{2}+\frac{\varepsilon}{2}=\varepsilon .
\end{aligned}
$$

Conversely, let $\left(a_{m, n}\right)$ be a Cauchy double sequence and consider the subsequences $\left(b_{n}\right)$ defined by $b_{n}:=\left(a_{n, n}\right)$ for all $n \in \mathbb{N}_{0}$. Therefore, by Cauchy's Criterion for single sequences, the sequence $\left(b_{n}\right)$ converges. Let $b_{n} \rightarrow b$ and let $\varepsilon>0$ be given. Then there is $n \in \mathbb{N}$ such that

$$
\left|b_{n}-b\right| \leq \frac{\varepsilon}{2} \quad \text { for all } \quad n \geq N
$$

Since $\left(a_{n, m}\right)$ is a Cauchy sequence, there exists $\left(N^{\prime}, N^{\prime}\right) \succeq(N, N)$ such that

$$
\left|a_{m, n}-a_{p, q}\right| \leq \frac{\varepsilon}{2} \quad \text { for all } \quad(m, n),(p, q) \succeq\left(N^{\prime}, N^{\prime}\right) .
$$

Then by (A.3) and (A.4) we have

$$
\begin{aligned}
\left|a_{m, n}-a\right| & \leq\left|a_{m, n}-a_{N^{\prime}, N^{\prime}}\right|+\left|b_{N}^{\prime}-b\right| \\
& <\frac{\varepsilon}{2}+\frac{\varepsilon}{2}=\varepsilon \text { for all }(m, n) \succeq\left(N^{\prime}, N\right) .
\end{aligned}
$$

Hence, $\left(a_{n, m}\right)$ converges to $b$.

To each double sequence $\left(a_{m, n}\right)$ there corresponds three important limits; namely:

(i) $\lim _{(m, n) \rightarrow(\infty, \infty)} a_{m, n}$.

(ii) $\lim _{m \rightarrow \infty}\left(\lim _{n \rightarrow \infty} a_{m, n}\right)$.

(iii) $\lim _{n \rightarrow \infty}\left(\lim _{m \rightarrow \infty} a_{m, n}\right)$.

The important question that is usually considered in this regard is the question of when can we interchange the order of the limit for a double sequence $a_{n, m}$; that is, when the limit (ii) above equals the limit (iii) above. The following result gives a necessary and sufficient condition for the existence of an iterated limit of a convergent double sequence:

Theorem A.1.3. [28] (Iterated Limits of Double Sequences). Suppose $\left(a_{m, n}\right)$ is a convergent double sequence and let $a_{m, n} \rightarrow$ a as $m \rightarrow \infty$ and $n \rightarrow \infty$.

(i) If $\lim _{n \rightarrow \infty} a_{m, n}$ exists for each $m \in \mathbb{N}_{0}$, then the iterated limit

$$
\lim _{m \rightarrow \infty}\left(\lim _{n \rightarrow \infty} a_{m, n}\right) \text {. }
$$

exists and it is equal to a. 
(ii) If $\lim _{m \rightarrow \infty} a_{m, n}$ exists for each $n \in \mathbb{N}_{0}$, then the iterated limit

$$
\lim _{n \rightarrow \infty}\left(\lim _{m \rightarrow \infty} a_{m, n}\right) .
$$

exists and it is equal to a

(iii) If the hypotheses in (i) and (ii) above hold, then the double sequence $\left(a_{m, n}\right)$ is bounded and

$$
\lim _{m \rightarrow \infty}\left(\lim _{n \rightarrow \infty} a_{m, n}\right)=a=\lim _{n \rightarrow \infty}\left(\lim _{m \rightarrow \infty} a_{m, n}\right) .
$$

Proof. Let $a_{m, n} \rightarrow a$ as $(m, n) \rightarrow(\infty, \infty)$ and let $\varepsilon>0$ be given, then there exists $\left(m_{0}, n_{0}\right) \in$ $\mathbb{N}_{0}^{2}$ such that

$$
\left|a_{m, n}-a\right|<\frac{\varepsilon}{2}, \quad \forall \quad(m, n) \succeq\left(m_{0}, n_{0}\right) .
$$

Assume that $\lim _{n \rightarrow \infty} a_{m, n}=b_{m}$ exists for each $m \in \mathbb{N}_{0}$. We need to show that $\lim _{m \rightarrow \infty} b_{m}=$ $a$ for each $m \in \mathbb{N}_{0}$. Let $\varepsilon>0$ be given, then for each $m \in \mathbb{N}_{0}$ there is $k_{m} \in \mathbb{N}_{0}$ such that

$$
\left|a_{m, n}-b_{m}\right|<\frac{\varepsilon}{2} \quad \forall \quad n \geq k_{m}
$$

Let $m \geq m_{0}$, if we let $n_{1}:=\max \left\{n_{0}, k_{m}\right\}$, then

$$
\left|b_{m}-a\right| \leq\left|b_{m}-a_{m, n_{1}}\right|+\left|a_{m, n_{1}}-a\right|<\frac{\varepsilon}{2}+\frac{\varepsilon}{2}=\varepsilon
$$

Hence, $b_{m} \rightarrow a$ as $m \rightarrow \infty$. This proves $(i)$. The proof of $(i i)$ is similar with an interchange of the $n$ and $m$ symbols.

Suppose now that the hypotheses in (i) and (ii) hold. Since $\left|a_{m, n}\right| \rightarrow|a|$, there exists $\left(m_{1}, n_{1}\right) \in \mathbb{N}_{0}^{2}$ such that

$$
\left|a_{m, n}\right|<1+|a| \quad \forall \quad(m, n) \succeq\left(m_{1}, n_{1}\right) .
$$

We give examples to show that if any of the hypotheses in the above proposition is not satisfied, then the conclusion(s) may not hold.

Example A.1.1. (i) Let

$$
a_{m, n}:=\left\{\begin{array}{lll}
(-1)^{m+n}\left(\frac{1}{m}+\frac{1}{n}\right) & \text { for } & (m, n) \in \mathbb{N}_{0}^{2} \backslash S \\
0 & \text { for } & (m, n) \in S
\end{array}\right.
$$


where $S=\{(0,0)\} \cup\{(m, 0)\} \cup\{(0, n)\}, m, n \in \mathbb{N}$. Since $\left|a_{m, n}\right| \leq 1 / m+1 / n$ for $(m, n) \in \mathbb{N}_{0}^{2} \backslash\{(0,0)\}$, we see that $a_{m, n} \rightarrow 0$. However, $\lim _{n \rightarrow \infty} a_{m, n}$ does not exist for any fixed $m \in \mathbb{N}$. Indeed,

$$
a_{m, n}=(-1)^{m}\left[(-1)^{n} / n+(-1)^{m} / m\right] \quad \text { for all } \quad(m, n) \in \mathbb{N}_{0}^{2} \backslash S .
$$

and $(-1)^{n} / n \rightarrow 0$ as $n \rightarrow \infty$, while $\lim _{n \rightarrow \infty}(-1)^{n} / m$ does not exist.

(ii) Let

$$
a_{m, n}:=\left\{\begin{array}{lll}
m n /\left(m^{2}+n^{2}\right) & \text { for } & (m, n) \in \mathbb{N}_{0}^{2} \backslash S \\
0 & \text { for } & (m, n) \in S
\end{array}\right.
$$

where $S=\{(0,0)\} \cup\{(m, 0)\} \cup\{(0, n)\}, m, n \in \mathbb{N}$. Then for each fixed $m \in \mathbb{N}$, $\lim _{n \rightarrow \infty} a_{m, n}$ exists and it is equal to 0 , since $\left|a_{m, n}\right| \leq m / n$ for all $n \in \mathbb{N}$. Similarly, for each fixed $n \in \mathbb{N}, \lim _{m \rightarrow \infty} a_{m, n}$ exists and it is equal to 0 . However, $\left(a_{m, n}\right)$ is not convergent, since $\left(a_{m, n}\right)=1 / 2$ if $m=n$ and $a_{m, n}=2 / 5$ if $m=2 n$.

(iii) Let

$$
a_{m, n}:=\left\{\begin{array}{lll}
m /(m+n) & \text { for } & (m, n) \in \mathbb{N}_{0}^{2} \backslash S \\
0 & \text { for } & (m, n) \in S
\end{array}\right.
$$

where $S=\{(0,0)\} \cup\{(m, 0)\} \cup\{(0, n)\}, m, n \in \mathbb{N}$. . Then for each fixed $m \in \mathbb{N}$, $\lim _{n \rightarrow \infty} a_{m, n}=0$, and for each fixed $m \in \mathbb{N}, \lim _{m \rightarrow \infty} a_{m, n}=1$. Hence, $\lim _{m \rightarrow \infty}\left(\lim _{n \rightarrow \infty} a_{m, n}\right)=$ 0 , whereas $\lim _{n \rightarrow \infty}\left(\lim _{m \rightarrow \infty} a_{m, n}\right)=1$. Notice that $\left(a_{m, n}\right)$ is not convergent, since $a_{m, n}=1 / 2$ if $m=n$ and $a_{m, n}=2 / 3$ if $m=2 n$.

\section{A.2 Monotone Double Sequences}

In this section, we define increasing and decreasing sequences of real numbers and we demonstrate a monotone convergence theorem for such sequences that are parallel to their counterparts for single sequences.

Definition A.2.1. [28]. Let $\left(a_{m, n}\right)$ be a double sequence of real numbers.

(i) If $a_{m, n} \leq a_{m+1, n}$ and $a_{m, n} \leq a_{m, n+1}$ for all $(m, n) \in \mathbb{N}_{0}^{2}$, we that say the sequence is monotonically increasing.

(ii) If $a_{m, n} \geq a_{m+1, n}$ and $a_{m, n} \geq a_{m, n+1}$ for all $(m, n) \in \mathbb{N}_{0}^{2}$, we that say the sequence is monotonically decreasing. 
Observe that a double sequence $\left(a_{m, n}\right)$ is monotonically increasing if and only if

$$
a_{m, n} \leq a_{p, q} \quad \text { for all } \quad(m, n),(p, q) \in \mathbb{N}_{0}^{2} \quad \text { with } \quad(m, n) \preceq(p, q) .
$$

Also, a double sequence $\left(a_{m, n}\right)$ is monotonically increasing if and only if for each fixed $m \in \mathbb{N}_{0}$, the sequence given by $n \rightarrow a_{m, n}$ is (monotonically) increasing and for each fixed $n \in \mathbb{N}_{0}$, the sequence given by $m \rightarrow a_{m, n}$ is (monotonically) increasing. Likewise for monotonically decreasing double sequences. A double sequence is said to be monotonic if it is monotonically increasing or monotonically decreasing.

Theorem A.2.1. [28]. (Monotone Convergence Theorem) A monotone double sequence of real numbers is convergent if and only if it is bounded. Further:

(i) A monotonically increasing double sequence $\left(a_{m, n}\right)$ is convergent if and only if it is bounded above. In this case,

$$
a_{m, n} \rightarrow \sup \left\{a_{m, n}:(m, n) \in \mathbb{N}_{0}^{2}\right\}
$$

If $\left(a_{m, n}\right)$ is monotonically increasing, but not bounded above, then $a_{m, n} \rightarrow \infty$.

(ii) A monotonically decreasing double sequence $\left(a_{m, n}\right)$ is convergent if and only if it is bounded below. In this case,

$$
a_{m, n} \rightarrow \inf \left\{a_{m, n}:(m, n) \in \mathbb{N}_{0}^{2}\right\}
$$

If $\left(a_{m, n}\right)$ is monotonically increasing, but not bounded below, then $a_{m, n} \rightarrow-\infty$.

Proof. Let $\left(a_{m, n}\right)$ be a monotonically increasing double sequence. Suppose it is bounded above, and let $a:=\sup \left\{a_{m, n}:(m, n) \in \mathbb{N}_{0}^{2}\right\}$. Given $\varepsilon>0$, there is $\left(m_{0}, n_{0}\right) \in \mathbb{N}_{0}^{2}$ such that $a-\varepsilon<a_{m_{0}, n_{0}}$ (then $a-\varepsilon$ is not an upper bound for the set $\sup \left\{a_{m, n}:(m, n) \in \mathbb{N}_{0}^{2}\right\}$ ). Hence

$$
a-\varepsilon<a_{m_{0}, n_{0}} \leq a_{m, n} \leq a<a+\varepsilon \quad \text { for all } \quad(m, n) \succeq\left(m_{0}, n_{0}\right) .
$$

Thus $a_{m, n} \rightarrow a$

Conversely, suppose $\left(a_{m, n}\right)$ is convergent and $a_{m, n} \rightarrow a$. Then there is $\left(m_{0}, n_{0}\right) \in \mathbb{N}_{0}^{2}$ such that

$$
a_{m, n}<a+1 \text { for all }(m, n) \succeq\left(m_{0}, n_{0}\right) .
$$

Now given any $(m, n) \in \mathbb{N}_{0}^{2}$, we have $\left(m+m_{0}, n+n_{0}\right) \succeq(m, n)$ as well as $\left(m+m_{0}, n+\right.$ $\left.n_{0}\right) \succeq\left(m_{0}, n_{0}\right)$, and so

$$
a_{m, n} \leq a_{m+m_{0}, n+n_{0}}<a+1 .
$$

Therefore, $\left(a_{m, n}\right)$ is bounded above by $a+1$. If $\left(a_{m, n}\right)$ is not bounded above, then given $\alpha \in \mathbb{R}$, there is $\left(m_{0}, n_{0}\right) \in \mathbb{N}_{0}^{2}$ such that $a_{m_{0}, n_{0}}>\alpha$. But then $a_{m, n} \geq a_{m_{0}, n_{0}}>\alpha$ for all $(m, n) \succeq\left(m_{0}, n_{0}\right)$. Thus $a_{m, n} \rightarrow \infty$. This completes the proof of $(i)$. A similar proof can be given for $(i i)$. 
Corollary A.2.1. A monotonic double sequence $\left(a_{m, n}\right)$ is convergent if and only if the sequence $\left(a_{p, p}\right)$ of its diagonal terms is convergent. In this case,

$$
\lim _{(m, n) \rightarrow(\infty, \infty)} a_{m, n}=\lim _{p \rightarrow \infty} a_{p, p} .
$$

Proof. Suppose $\left(a_{m, n}\right)$ is a monotonically decreasing sequence. For any $(m, n) \in \mathbb{N}_{0}^{2}$ we let $p:=\max \{m, n\}$, then $a_{m, n} \geq a_{p, p}$. Consequently, $\left\{a_{m, n}:(m, n) \in \mathbb{N}_{0}^{2}\right\}$ is bounded below if and only if $\left\{a_{p, p}: p \in \mathbb{N}_{0}\right\}$ is bounded below, and in this case, $\inf \left\{a_{m, n}:(m, n) \in\right.$ $\left.\mathbb{N}_{0}^{2}\right\}=\inf \left\{a_{p, p}: p \in \mathbb{N}_{0}\right\}$. Hence Theorem A.2.1 yields the desired result. The case of when $\left(a_{m, n}\right)$ is a monotonically increasing double sequence is proved similarly.

\section{A.3 Convergence of Double Series}

Let $\left(a_{k, \ell}\right)$ be a double sequence of complex numbers, we define $\sum \sum_{(k, \ell)} a_{k, \ell}$ as a double indexed infinite series of complex numbers. We define the associated sequence of partial sums $\left(s_{m, n}\right)$ via the finite sum

$$
s_{m, n}=\sum_{k=0}^{m} \sum_{\ell=0}^{n} a_{k, \ell}=\sum_{\substack{0 \leq k \leq m \\ 0 \leq \ell \leq n}} a_{k, \ell}=\left(a_{0,0}+\cdots+a_{0, n}\right)+\cdots+\left(a_{m, 0}+\cdots+a_{m, n}\right),
$$

for all $(m, n) \in \mathbb{N}_{0}^{2}$. Moreover, we have

$$
a_{k, \ell}=s_{k, \ell}-s_{k, \ell-1}-s_{k-1, \ell}+s_{k-1, \ell-1} \quad \forall \quad(k, \ell) \in \mathbb{N}_{0}^{2},
$$

where $s_{k,-1}:=0$ for all $k=0,1,2, \ldots$ and $s_{-1, \ell}:=0$ for all $\ell=0,1,2, \ldots$.

We say that a double series $\sum \sum_{(k, \ell)} a_{k, \ell}$ is convergent to the sum $s$ if $\lim _{m, n \rightarrow \infty} s_{m, n}=s$. If no such limit exists, we say that the double series $\sum \sum_{(k, \ell)} a_{k, \ell}$ is divergent.

By considering the real and imaginary parts, we see that the series $\sum \sum_{(k, \ell)} a_{k, \ell}$ converges if and only if both $\sum \sum_{(k, \ell)} \operatorname{Re}\left(a_{k, \ell}\right)$ and $\sum \sum_{(k, \ell)} \operatorname{Im}\left(a_{k, \ell}\right)$ converge. If this is the case, then

$$
\sum \sum_{(k, \ell)} a_{k, \ell}=\sum \sum_{(k, \ell)} \operatorname{Re}\left(a_{k, \ell}\right)+i\left(\sum \sum_{(k, \ell)} \operatorname{Im}\left(a_{k, \ell}\right)\right) .
$$

Indeed, for any $(m, n) \in \mathbb{N}_{0}^{2}, s_{m, n}=\operatorname{Re} s_{m, n}+i \operatorname{Im} s_{m, n}$, and $s_{m, n}$ converges if and only if both $s_{m, n}=\operatorname{Re} s_{m, n}$ and $s_{m, n}=\operatorname{Im} s_{m, n}$ converge.

As with single series, a simple and useful way to demonstrate that a double series is divergent is to use the following test, which gives a necessary condition for a double series convergence: 
Theorem A.3.1. $\left((k, \ell)\right.$ th Term Test) [28]. If $\sum \sum_{(k, \ell)} a_{k, \ell}$ is convergent, then $a_{k, \ell} \rightarrow 0$ as $k, \ell \rightarrow \infty$. In other words, if $a_{k, \ell} \nrightarrow 0$ as $k, \ell \rightarrow \infty$, then $\sum \sum_{(k, \ell)} a_{k, \ell}$ is divergent.

Proof. Let $\sum \sum_{(k, \ell)} a_{k, \ell}$ be a convergent double series. If $\left(s_{k, \ell}\right)$ is the double sequence of its partial double sums, such that $s_{k, \ell} \rightarrow a$ as $(k, \ell) \rightarrow(\infty, \infty)$. So given $\varepsilon>0$, there exists $\left(k_{0}, \ell_{0}\right) \in \mathbb{N}_{0}^{2}$ such that

$$
\left|s_{k, \ell}-a\right| \leq \frac{\varepsilon}{4} \quad \forall \quad(k, \ell) \succeq\left(k_{0}, \ell_{0}\right),
$$

then we have

$$
\begin{aligned}
\left|a_{k, \ell}\right| & =\left|s_{k, \ell}-s_{k, \ell-1}-s_{k-1, \ell}+s_{k-1, \ell-1}\right| \\
& \leq\left|s_{k, \ell}-a\right|+\left|s_{k, \ell-1}-a\right|+\left|s_{k-1, \ell}-a\right|+\left|s_{k-1, \ell-1}-a\right| \\
& <\frac{\varepsilon}{4}+\frac{\varepsilon}{4}+\frac{\varepsilon}{4}+\frac{\varepsilon}{4}=\varepsilon .
\end{aligned}
$$

Therefore, $a_{k, \ell} \rightarrow 0$ as $(k, \ell) \rightarrow(\infty, \infty)$.

The following result gives a sufficient condition for the convergence of certain "product series," and is often helpful:

Theorem A.3.2. [28]. Let $\sum_{k} b_{k}$ and $\sum_{\ell} c_{\ell}$ be series of real numbers and let $a_{k, \ell}=b_{k} c_{\ell}$ for $(k, \ell) \in \mathbb{N}_{0}^{2}$. Then the following results hold:

(i) If $\sum_{k} b_{k}$ and $\sum_{\ell} c_{\ell}$ are both convergent, then the double series $\sum \sum_{(k, \ell)} a_{(k, \ell)}$ is convergent and moreover, $\sum \sum_{(k, \ell)} a_{(k, \ell)}=\sum_{k} b_{k} \sum_{\ell} c_{\ell}$.

(ii) If $\sum_{k} b_{k}$ and $\sum_{\ell} c_{\ell}$ are both diverge to $\infty$, then the double series $\sum \sum_{(k, \ell)} a_{(k, \ell)}$ diverges to $\infty$.

(iii) If $\sum_{k} b_{k}$ converges to $B \neq 0$, while $\sum_{\ell} c_{\ell}$ is divergent, then the double series $\sum \sum_{(k, \ell)} a_{(k, \ell)}$ is divergent.

Proof. Let $\left(B_{m}\right)$ and $\left(C_{n}\right)$ denote the sequences of partial sums of the series $\sum_{k} b_{k}$ and $\sum_{\ell} c_{\ell}$ respectively. Also, let $\left(s_{m, n}\right)$ denote the double sequence of partial double sums of $\sum \sum_{(k, \ell)} a_{(k, \ell)}$. Then

$$
s_{m, n}=\left(\sum_{k=0}^{m} b_{k}\right)\left(\sum_{\ell=0}^{n} c_{\ell}\right)=B_{m} C_{n} \quad \forall \quad(m, n) \in \mathbb{N}_{0}^{2} .
$$

Therefore, if $B_{m} \rightarrow b$ and $C_{m} \rightarrow c$ for all $b, c \in \mathbb{C}$, then $s_{m, n} \rightarrow b c$. Also, if $B_{m} \rightarrow \infty$ and $C_{m} \rightarrow \infty$, then $s_{m, n} \rightarrow \infty$. This proves ( $i$ ) and (ii) Moreover, if $B_{m} \rightarrow b$ with $b \neq 0$ and if the double sequence $\left(s_{m, n}\right)$ converges to $s$, then $\left(C_{n}\right)$ converges to $s / b$. This proves (iii). 
Example A.3.1. [28].

(i) (Geometric Double Series) Let $x, y \in \mathbb{C}$. Define $a_{k, \ell}:=x^{k} y^{\ell}$ for $k, \ell \in \mathbb{N}_{0}$. The double series $\sum \sum_{(k, \ell)} a_{(k, \ell)}$, where the index $(k, \ell)$ varies over pairs of non-negative integers is called the geometric double series. Hence from part (i) of Theorem A.3.2, we see that the geometric double series is convergent if $|x|<1$ and $|y|<1$; moreover,

$$
\begin{aligned}
\sum \sum_{(k, \ell)} a_{k, \ell} & =\sum \sum_{(k, \ell) \succeq(0,0)} x^{k^{k} y^{\ell}} \\
& =\frac{1}{(1-x)(1-y)} \text { for } \quad|x|<1 \quad \text { and } \quad|y|<1
\end{aligned}
$$

Further, if $|x| \geq 1$ and $|y| \geq 1$, then $\left|x^{k} y^{\ell}\right|=|x|^{k}|y|^{\ell} \geq 1$ for all $k, l \in \mathbb{N}_{0}$. Hence from the $(k, l)$ th Term Test (Theorem A.3.1), we see that the geometric double series is divergent. Finally, since $\frac{1}{1-z}$ is nonzero whenever $z \in \mathbb{C}$ with $|z|<1$, it follows from part (iii) of Theorem A.3.2 that if only one of $|x|$ and $|y|$ is less than 1, then the geometric double series is divergent. Thus we see that geometric double series $\sum \sum_{(k, \ell)} a_{k, \ell}$ is convergent if and only if $|x|<1$ and $|y|<1$.

(ii) (Exponential Double Series) Let $x, y \in \mathbb{C}$. Define $a_{k, \ell}=\frac{x^{k} y^{\ell}}{k ! \ell !}$ for $k, \ell \in \mathbb{N}_{0}$. The double series $\sum \sum_{(k, \ell)} a_{k, \ell}$, is called the exponential double series. From part $(i)$ of Theorem A.3.2, we readily see that the exponential double series is always convergent and

$$
\begin{aligned}
& \sum \sum_{(k, \ell)} a_{k, \ell}=\sum \sum_{(k, \ell) \succeq(0,0)} \frac{x^{k}}{k !} \frac{y^{\ell}}{\ell !} \\
& =\exp (x) \exp (y)=\exp (x+y) \quad \text { for } \quad x, y \in \mathbb{C} .
\end{aligned}
$$

(iii) (Harmonic Double Series and Their Variants) The double series

$\sum \sum_{(k, \ell)} \frac{1}{(k+1)(\ell+1)}$ can be regarded as analogues of the harmonic series $\sum_{k=0} \frac{1}{k+1}$, and either of the two double series may be referred to as a harmonic double series. We know from the theory of (single) series that the harmonic series diverges to $\infty$. Hence by part (ii) of Theorem A.3.2, we see that the double series $\sum \sum_{(k, \ell)} \frac{1}{(k+1)(\ell+1)}$ diverges to $\infty$. More generally, for any $p \in \mathbb{R}$, we know that the series $\sum_{k=0} 1 /(k+1)^{p}$ is convergent for $p>1$ and it diverges to $\infty$ for $p \leq 1$. Thus, using parts (i), (ii), and (iii) of Theorem A.3.2, we see that for any $p, q \in \mathbb{R}$,

$$
\sum_{(k, \ell)} \frac{1}{(k+1)^{p}(1+\ell)^{q}} \quad \Longleftrightarrow \quad p>1 \text { and } q>1 \text {. }
$$


As for the other version of the harmonic series, namely the double series $\sum \sum_{(k, \ell)} 1(k+$ $\ell+2)$, we also find that it diverges to $\infty$, since

$$
\sum_{k=0}^{m} \sum_{\ell=0}^{n} \frac{1}{(k+\ell+2)} \geq \frac{1}{2} \sum_{k=0}^{m} \sum_{\ell=0}^{n} \frac{1}{(k+1)(\ell+1)} \quad \text { for all } \quad(m, n) \in \mathbb{N}_{0}^{2} .
$$

Next, we consider the series $\sum \sum_{(k, \ell)} 1 /(k+\ell+2)^{2}$. For $n \in \mathbb{N}_{0}$, the terms of a double sequences $\left(a_{k, \ell}\right)=1 /(k+\ell+2)^{2}$ are given schematically as follows:

$$
\left(a_{k, \ell}\right)=\begin{array}{ccccccc}
\frac{1}{4} & \frac{1}{9} & \frac{1}{16} & \frac{1}{25} & \frac{1}{36} & \frac{1}{49} & \ldots \\
\frac{1}{9} & \frac{1}{16} & \frac{1}{25} & \frac{1}{36} & \frac{1}{49} & \frac{1}{64} & \ldots \\
\frac{1}{16} & \frac{1}{25} & \frac{1}{36} & \frac{1}{49} & \frac{1}{64} & \frac{1}{81} & \ldots \\
\frac{1}{25} & \frac{1}{36} & \frac{1}{49} & \frac{1}{64} & \frac{1}{81} & \frac{1}{100} & \ldots \\
\frac{1}{36} & \frac{1}{49} & \frac{1}{64} & \frac{1}{81} & \frac{1}{100} & \frac{1}{121} & \ldots \\
\frac{1}{49} & \frac{1}{64} & \frac{1}{81} & \frac{1}{100} & \frac{1}{121} & \frac{1}{144} & \ldots \\
\vdots & \vdots & \vdots & \vdots & \vdots & \vdots & \ddots
\end{array}
$$

Summing the terms $\left(a_{k, \ell}\right)=1 /(k+\ell+2)^{2}$ diagonally shows that

$$
\sum_{(k, \ell)} \sum_{1} 1 /(k+\ell+2)^{2}=\sum_{n=2}^{\infty} \frac{n-1}{n^{2}} .
$$

Thus the double series $\sum \sum_{(k, \ell)} 1 /(k+\ell+2)^{2}$ diverges to $\infty$. This indicates that the threshold for the convergence of $\sum \sum_{(k, \ell)} 1 /(k+\ell+2)^{p}$ is not $p=1$.

The following statements concerning the convergence of a double series are extracted from the corresponding statements for the convergence of a double sequence given in Section A.1:

(i) (Limit Theorem) Let $\sum \sum_{(k, \ell)} a_{k, \ell}=A$ and $\sum \sum_{(k, \ell)} b_{k, \ell}=B$. Then $\sum \sum_{(k, \ell)}\left(a_{k, \ell}+\right.$ $\left.a_{k, \ell}\right)=A+B$ and $\sum \sum_{(k, \ell)}\left(r a_{k, \ell}\right)=r A$ for any $r \in \mathbb{C}$. Further, if $a_{k, \ell} \leq b_{k, \ell}$ for all $(k, \ell) \in \mathbb{N}^{2}$, then $A \leq B$.

(ii) (Sandwich Theorem) If $\left(a_{k, l}\right),\left(b_{k, l}\right)$, and $\left(c_{k, l}\right)$ are double sequences of real numbers such that $a_{k, l} \leq c_{k, l} \leq b_{k, l}$ for each $(k, \ell) \in \mathbb{N}_{0}^{2}$, and further if $\sum \sum_{(k, \ell)} a_{k, \ell}=A$ as well as $\sum \sum_{(k, \ell)} b_{k, \ell}=A$, then $\sum \sum_{(k, \ell)} c_{k, \ell}=A$.

(iii) (Cauchy Criterion) Consider the associated sequence $s_{m, n}=\sum_{k=0}^{m} \sum_{\ell=0}^{n} a_{k, \ell}$ of partial sums of $\sum \sum_{(k, \ell)} a_{k, \ell}$. the given series converges if and only if its sequences of partial summs $s_{m, n}$ is convergent and that is convergent if and only if it is Cauchy 
(Theorem A.1.2). Thus $\sum \sum_{(k, \ell)} a_{k, \ell}$ is convergent if and only if for $\varepsilon>0$ there exists $\left(m_{0}, n_{0}\right) \in \mathbb{N}_{0}^{2}$ such that

$$
\left|s_{p, q}-s_{m, n}\right|<\varepsilon \quad \text { for all } \quad(m, n) \succeq(p, q) \succeq\left(m_{0}, n_{0}\right) .
$$

But for $(m, n) \succeq(p, q)$, we have

$$
\left|s_{p, q}-s_{m, n}\right|=\left|\sum_{k=p+1}^{m} \sum_{\ell=q+1}^{n} a_{k, \ell}+\sum_{k=1}^{p} \sum_{\ell=1}^{n} a_{k, \ell}+\sum_{k=p+1}^{m} \sum_{\ell=1}^{q} a_{k, \ell}\right|<\varepsilon .
$$

We will now relate the convergence of a double series $\sum \sum_{(k, \ell)} a_{k, \ell}$ to the convergence of the two series $\sum_{k=0}^{\infty}\left(\sum_{\ell=0}^{\infty} a_{k, \ell}\right)$ and $\sum_{\ell=0}^{\infty}\left(\sum_{k=0}^{\infty} a_{k, \ell}\right)$. For each fixed $k \in \mathbb{N}_{0}$, the (single) series $\sum_{\ell} a_{k, \ell}$ is called a row-series, and for each fixed $\ell \in \mathbb{N}_{0}$, the (single) series $\sum_{k} a_{k, \ell}$ is called a column-series (corresponding to the double series $\sum \sum_{(k, \ell)} a_{k, \ell}$ ).

\section{Theorem A.3.3. [28]. (Fubini's Theorem for Double Series)}

Assume that $\sum \sum_{(k, \ell)} a_{k, \ell}$ is a convergent double series and let $s$ denote its double sum.

(i) If each row-series is convergent, then the corresponding iterated series $\sum_{k=0}^{\infty}\left(\sum_{\ell=0}^{\infty} a_{k, \ell}\right)$ is convergent and its sum is equal to s.

(ii) If each column-series is convergent, then the corresponding iterated series $\sum_{\ell=0}^{\infty}\left(\sum_{k=0}^{\infty} a_{k, \ell}\right)$ is convergent and its sum is equal to s.

(iii) If each row-series as well as each column-series is convergent, then the double sequence of partial double sums of $\sum \sum_{(k, \ell)} a_{k, \ell}$ is bounded, and

$$
\sum_{k=0}^{\infty}\left(\sum_{\ell=0}^{\infty} a_{k, \ell}\right)=\sum \sum_{(k, \ell)} a_{k, \ell}=\sum_{\ell=0}^{\infty}\left(\sum_{k=0}^{\infty} a_{k, \ell}\right)
$$

Proof. Let $s_{m, n}$ be the double sequence of partial double sums of $\sum \sum_{(k, \ell)} a_{k, \ell}$. By our assumption, $s_{m, n} \rightarrow s$.

Suppose each row-series is convergent. Then for each fixed $m \in \mathbb{N}_{0}$,

$$
s_{m}:=\lim _{n \rightarrow \infty} s_{m, n}=\lim _{n \rightarrow \infty} \sum_{k=0}^{m} \sum_{\ell=0}^{n} a_{k, \ell}=\sum_{k=0}^{m}\left(\lim _{n \rightarrow \infty} \sum_{\ell=0}^{n} a_{k, \ell}\right)=\sum_{k=0}^{m}\left(\sum_{\ell=0}^{\infty} a_{k, \ell}\right) .
$$

Hence by Theorem A.1.3, the iterated $\operatorname{limit} \lim _{m \rightarrow \infty}\left(\lim _{n \rightarrow \infty} s_{m, n}\right)$ exists and is equal to $s$, that is,

$$
\lim _{m \rightarrow \infty} \sum_{k=0}^{m}\left(\sum_{\ell=0}^{\infty} a_{k, \ell}\right)=s
$$


Thus the iterated series $\lim _{m \rightarrow \infty}\left(\lim _{n \rightarrow \infty} s_{m, n}\right)$ converges and its sum equals $s$. This proves $(i)$. The proof of (ii) is similar.

Finally, suppose each row-series as well as each column-series is convergent. Then

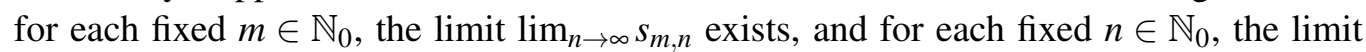
$\lim _{m \rightarrow \infty} s_{m, n}$ exists. Hence by part (iii) of Theorem A.1.3, $s_{m, n}$ is bounded. The last part of (iii) follows from (i) and (ii).

Example A.3.2. [28].

(i) Even if a double series $\sum \sum_{(k, \ell)} a_{k, \ell}$ converges, both the iterated series may diverge. For instance, consider a double sequences $\left(a_{k, l}\right),\left(s_{m, n}\right)$ given schematically as follows:

$$
\begin{aligned}
& \begin{array}{llllll}
1 & 1 & 1 & 1 & \ldots
\end{array} \\
& \begin{array}{lllll}
1 & -3 & -1 & -1 & \ldots
\end{array} \\
& \left(a_{k, \ell}\right)=\begin{array}{lllll}
1 & -1 & 0 & 0 & \ldots \\
1 & -1 & 0 & 0 & \ldots
\end{array} \\
& \vdots \quad \vdots \quad \vdots \quad \vdots \quad \ddots \\
& \begin{array}{lllllll}
1 & 2 & 3 & 4 & \ldots & \rightarrow & \infty
\end{array} \\
& \begin{array}{lllllll}
2 & 0 & 0 & 0 & \ldots & \rightarrow & 0
\end{array} \\
& \begin{array}{lllllll}
3 & 0 & 0 & 0 & \ldots & \rightarrow & 0
\end{array} \\
& \left(s_{m, n}\right)=\begin{array}{lllllll}
4 & 0 & 0 & 0 & \ldots & \rightarrow & 0
\end{array} \\
& \vdots \quad \vdots \quad \vdots \quad \ddots \quad \downarrow \\
& \downarrow \downarrow \downarrow \downarrow \downarrow \searrow 0
\end{aligned}
$$

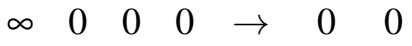

Then $s_{0, n}=n$ for all $n \in \mathbb{N}_{0}$ and $s_{m, 0}=m$ for all $m \in \mathbb{N}_{0}$, while $s_{m, n}=0$ for all $m, n>0$. Hence $\sum \sum_{(k, \ell)} a_{k, \ell}=\lim _{(m, n) \rightarrow(\infty, \infty)} s_{m, n}=0$. But $\sum_{\ell=0}^{n} a_{0, \ell}=n$ for all $n \in \mathbb{N}$ and $\sum_{\ell=0}^{n} a_{1, \ell}=-n$ for all $n \geq 1$, while $\sum_{k=0}^{m} a_{k, 0}=m$ for all $m \in \mathbb{N}$ and $\sum_{k=0}^{m} a_{k, 1}=-m$ or all $m \geq 1$. Hence $\sum_{\ell=0}^{\infty} a_{0, \ell}$ and $\sum_{k=0}^{\infty} a_{k, 0}$ diverge to $\infty$, whereas $\sum_{\ell=0}^{\infty} a_{1, \ell}$ and $\sum_{k=0}^{\infty} a_{k, 1}$ diverge to $-\infty$. Clearly, none of the iterated series is even well defined.

(ii) Even if both iterated series $\sum_{k=0}^{\infty}\left(\sum_{\ell=0}^{\infty} a_{k, \ell}\right)$ and $\sum_{\ell=0}^{\infty}\left(\sum_{k=0}^{\infty} a_{k, \ell}\right)$ converge and have the same sum, the double series may diverge. For example, consider a double 
sequences $\left(a_{k, \ell}\right),\left(s_{m, n}\right)$ given schematically as follows:

$$
\begin{aligned}
& \left(a_{k, \ell}\right)=\begin{array}{ccccccc}
2 & 0 & -1 & 0 & 0 & 0 & \ldots \\
0 & 2 & 0 & -1 & 0 & 0 & \ldots \\
-1 & 0 & 2 & 0 & -1 & 0 & \ldots \\
0 & -1 & 0 & 2 & 0 & -1 & \ldots \\
0 & 0 & -1 & 0 & 2 & 0 & \ldots \\
\vdots & \vdots & \vdots & \vdots & \vdots & \ddots &
\end{array} \\
& \left(s_{m, n}\right)=\begin{array}{ccccccccc}
2 & 2 & 1 & 1 & 1 & 1 & \ldots & \rightarrow & 1 \\
2 & 4 & 3 & 2 & 2 & 2 & \ldots & \rightarrow & 2 \\
1 & 3 & 4 & 3 & 2 & 2 & \ldots & \rightarrow & 2 \\
1 & 2 & 3 & 4 & 3 & 2 & \ldots & \rightarrow & 2 \\
1 & 2 & 2 & 3 & 4 & 3 & \ldots & \rightarrow & 2 \\
\vdots & \vdots & \vdots & \vdots & \vdots & \vdots & \ddots & & \downarrow \\
\downarrow & \downarrow & \downarrow & \downarrow & \downarrow & \downarrow & & \searrow & 2 \\
1 & 2 & 2 & 2 & 2 & 2 & \rightarrow & 2 & 4
\end{array}
\end{aligned}
$$

Then $\sum_{\ell=0}^{\infty} a_{k, \ell}$ is equal to 1 if $k=0$ or 1 , and it is equal to 0 if $k \geq 2$. Similarly, $\sum_{k=0}^{\infty} a_{k, \ell}$ is equal to 1 if $\ell=0$ or 1 , and it is equal to 0 if $\ell \geq 2$. Thus $\sum_{k=0}^{\infty}\left(\sum_{\ell=0}^{\infty} a_{k, \ell}\right)=2=\sum_{\ell=0}^{\infty}\left(\sum_{k=0}^{\infty} a_{k, \ell}\right)$. But $s_{m, n}=4$ for all $m=n \geq 1$ and $s_{m, m-1}=$ 3 for all $m \geq 2$, so that the double sequence $\left(s_{m, n}\right)$ is divergent, that is, the double series $\sum \sum_{(k, \ell)} a_{k, \ell}$ is divergent.

(iii) Even if both iterated series $\sum_{k=0}^{\infty}\left(\sum_{\ell=0}^{\infty} a_{k, \ell}\right)$ and $\sum_{\ell=0}^{\infty}\left(\sum_{k=0}^{\infty} a_{k, \ell}\right)$ converge, their sums may be unequal. For example, consider a double sequences $\left(a_{k, l}\right),\left(s_{m, n}\right)$ given schematically as follows:

$$
\left(a_{k, \ell}\right)=\begin{array}{cccccc}
0 & 1 & 0 & 0 & 0 & \ldots \\
-1 & 0 & 1 & 0 & 0 & \ldots \\
0 & -1 & 0 & 1 & 0 & \ldots \\
0 & 0 & -1 & 0 & 1 & \ldots \\
\vdots & \vdots & \vdots & \vdots & \vdots & \ddots
\end{array}
$$




$$
\left(s_{m, n}\right)=\begin{array}{ccccccc}
0 & 1 & 1 & 1 & \ldots & \rightarrow & 1 \\
-1 & 0 & 1 & 1 & \ldots & \rightarrow & 1 \\
-1 & -1 & 0 & 1 & \ldots & \rightarrow & 1 \\
-1 & -1 & -1 & 0 & \ldots & \rightarrow & 1 \\
\vdots & \vdots & \vdots & \vdots & \ddots & & \downarrow \\
\downarrow & \downarrow & \downarrow & \downarrow & & \searrow & 1 \\
-1 & -1 & -1 & -1 & \rightarrow & -1 & 0
\end{array}
$$

Then $\sum_{\ell=0}^{\infty} a_{k, \ell}$ is equal to 1 if $k=0$ and it is equal to 0 if $k \geq 1$, whereas $\sum_{k=0}^{\infty} a_{k, \ell}$ is equal to -1 if $\ell=0$ and it is equal to 0 if $\ell \geq 1$. Hence $\sum_{k=0}^{\infty}\left(\sum_{\ell=0}^{\infty} a_{k, \ell}\right)=1$, while $\sum_{\ell=0}^{\infty}\left(\sum_{k=0}^{\infty} a_{k, \ell}\right)=-1$. Since $s_{m, m}=0$ for all $m \in \mathbb{N}_{0}, s_{m, n}=1$ for all $m<n$ and $s_{m, n}=-1$ for all $m>n$, then $\sum \sum_{(k, \ell)} a_{k, \ell}$ is divergent.

\section{A.4 Cauchy Product of Double Series}

The Cauchy product of sequences $\left(a_{k}\right)$ and $\left(b_{k}\right)$ with $k \in \mathbb{N}_{0}$, is defined to be the sequence $\left(a_{k} * b_{k}\right)$, where $a_{k} * b_{k}:=\sum_{i=0}^{k} a_{i} b_{k-i}$ for $k \in \mathbb{N}_{0}$ and the Cauchy product of single series $\sum_{k=0} a_{k}$ and $\sum_{k=0} b_{k}$ is defined to be the double series $\sum_{k=0} a_{k} * b_{k}$. Analogously, the Cauchy product of double sequences $\left(a_{k, \ell}\right)$ and $\left(b_{k, \ell}\right)$ with $(k, \ell) \in \mathbb{N}_{0}^{2}$, is defined to be the sequence $\left(a_{k, \ell} * b_{k, \ell}\right)$ defined as

$$
\left(a_{k, \ell} * b_{k, \ell}\right)=\sum_{i=0}^{k} \sum_{j=0}^{\ell} a_{i, j} b_{k-i, \ell-j} \quad \text { for all } \quad(k, \ell) \in \mathbb{N}_{0}^{2},
$$

and the Cauchy product of double series $\sum_{k, \ell=0}^{\infty} a_{k, \ell}$ and $\sum_{k, \ell=0}^{\infty} b_{k, \ell}$ is defined to be the double series $\sum_{k, \ell=0}^{\infty} a_{k, \ell} * b_{k, \ell}$. A classical result of Mertens states that if one of the given single series is absolutely convergent and the other is convergent, then their Cauchy product series is convergent. Another result due to Abel states that if both the given single series and their Cauchy product series are convergent, then the sum of the Cauchy product series is equal to the product of the sums of the given series. It has been known for long that the exact analogue of Mertens' result does not hold for double series. The example below shows that the exact analogue of Abel's result does not hold for double series.

Example A.4.1. Consider a double sequences $\left(a_{k, \ell}\right),\left(b_{k, \ell}\right)$ given schematically as follows:

$$
\left(a_{k, \ell}\right)=\begin{array}{cccccc}
1 & 1 & 1 & 1 & 1 & \ldots \\
-1 & -1 & -1 & -1 & -1 & \ldots \\
0 & 0 & 0 & 0 & 0 & \ldots \\
0 & 0 & 0 & 0 & 0 & \ldots
\end{array}
$$




$$
\left(b_{k, \ell}\right)=\begin{array}{cccccc}
1 & -1 & 0 & 0 & 0 & \ldots \\
1 & -1 & 0 & 0 & 0 & \ldots \\
1 & -1 & 0 & 0 & 0 & \ldots \\
1 & -1 & 0 & 0 & 0 & \ldots \\
\vdots & \vdots & \vdots & \vdots & \vdots & \ddots
\end{array}
$$

Then, the double series $\sum_{k, \ell=0} a_{k, \ell}, \sum_{k, \ell=0} b_{k, \ell}$ are convergent, and the sum of each is equal to 0 . Also, it is easy to see that

$$
\left(a_{k, \ell} * b_{k, \ell}\right)=\begin{array}{cccccc}
1 & 0 & 0 & 0 & 0 & \ldots \\
0 & 0 & 0 & 0 & 0 & \ldots \\
0 & 0 & 0 & 0 & 0 & \ldots \\
0 & 0 & 0 & 0 & 0 & \ldots \\
\vdots & \vdots & \vdots & \vdots & \vdots & \ddots
\end{array}
$$

so that $\sum_{k, \ell=0} a_{k, \ell} * b_{k, \ell}$ is convergent, and its sum is 1 .

\section{A.5 Double Series with Non-negative Terms}

The following necessary and sufficient condition for the convergence of a double series with non-negative terms is very useful:

Theorem A.5.1. [28]. Let $\left(a_{k, l}\right)$ be a real double sequence such that $a_{k, l} \geq 0$ for all $(k, l) \in \mathbb{N}_{0}^{2}$. Then $\sum \sum_{(k, \ell)} a_{k, \ell}$ is convergent if and only if the double sequence $\left(s_{m, n}\right)$ of its partial double sums is bounded above, and in this case

$$
\sum \sum_{(k, \ell)} a_{k, \ell}=\sup \left\{s_{m, n}:(m, n) \in \mathbb{N}_{0}^{2}\right\}
$$

If $\left(s_{m, n}\right)$ is not bounded above, then $\sum \sum_{(k, \ell)} a_{k, \ell}$ diverges to $\infty$.

Proof. Let $\sum \sum_{(k, \ell)} a_{k, \ell}$ be a double series with $a_{k, \ell} \geq 0 \quad \forall \quad(k, \ell) \in \mathbb{N}_{0}^{2}$, then we have $s_{m+1, n}=s_{m, n}+a_{m+1,1}+\cdots+a_{m+1, n} \geq s_{m, n}$ and $s_{m, n+1}=s_{m, n}+a_{1, n+1}+\cdots+a_{m, n+1} \geq s_{m, n}$ for all $(m, n) \in \mathbb{N}_{0}^{2}$. Hence the double sequence $\left(s_{m, n}\right)$ is monotonically increasing. By part (i) of Theorem (A.2.1), we see that $\left(s_{m, n}\right)$ is convergent if and only if it is bounded above, and in this case

$$
\sum \sum_{(k, \ell)} a_{k, \ell}=\lim _{(m, n) \rightarrow(\infty, \infty)} s_{m, n}=\sup \left\{s_{m, n}:(m, n) \in \mathbb{N}_{0}^{2}\right\} .
$$

Moreover, if $\left(s_{m, n}\right)$ is not bounded above, then $s_{m, n} \rightarrow \infty$, that is, the double series $\sum \sum_{(k, \ell)} a_{k, \ell}$ diverges to $\infty$. 
The previous theorem shows that if a double series $\sum \sum_{(k, \ell)} a_{k, \ell}$ with non-negative terms is convergent and $s$ is its double sum, then the double sequence $\left(a_{k, l}\right)$ of its terms as well as the double sequence $\left(s_{m, n}\right)$ of its partial double sums is bounded. This follows by observing that in this case, $0 \leq a_{k, l} \leq s_{k, l} \leq s$ for all $(k, \ell) \in \mathbb{N}_{0}^{2}$.

Corollary A.5.1. [28]. A double series of non-negative terms $\sum \sum_{(k, \ell)} a_{k, \ell}$ either converges to a finite number $s$ or else it diverges properly to $\infty$.

A result similar to Theorem A.5.1 holds for double series with non-positive terms. More generally, when the terms $a_{k, l}$ have the same sign except possibly for a finite number of them, then $\sum \sum_{(k, \ell)} a_{k, \ell}$ is convergent if and only if $\left(s_{m, n}\right)$ is bounded. However, if infinitely many $a_{k, l}$ 's are positive and infinitely many $a_{k, l}$ 's are negative, then $\sum \sum_{(k, \ell)} a_{k, \ell}$ may diverge even though $\left(s_{m, n}\right)$ is bounded, and $\left(s_{m, n}\right)$ may be unbounded even though $\sum \sum_{(k, \ell)} a_{k, \ell}$ is convergent. These two statements are illustrated respectively by the double series given schematically as follows:

$$
\left(a_{k, \ell}\right)=\begin{array}{ccccc}
1 & 0 & 0 & 0 & \ldots \\
-1 & 0 & 0 & 0 & \ldots \\
1 & 0 & 0 & 0 & \ldots \\
-1 & 0 & 0 & 0 & \ldots
\end{array} \quad \text { and } \quad\left(b_{k, \ell}\right)=\begin{array}{rrrrr}
1 & -1 & 0 & 0 & \ldots \\
1 & -1 & 0 & 0 & \ldots \\
1 & -1 & 0 & 0 & \ldots \\
1 & -1 & 0 & 0 & \ldots
\end{array}
$$

For double series with nonnegative terms, the following result is an improvement over Fubini's Theorem for double series (Theorem A.3.3):

Theorem A.5.2. (Tonelli's Theorem for Double Series)[28]. Let $\left(a_{k, l}\right)$ be a double sequence such that $a_{k, l} \geq 0$ for all $(k, l) \in \mathbb{N}_{0}^{2}$. Then the following statements are equivalent.

(i) The double series $\sum \sum_{(k, \ell)} a_{k, \ell}$ is convergent.

(ii) Each row-series is convergent and the iterated series $\sum_{k=0}^{\infty}\left(\sum_{\ell=0}^{\infty} a_{k, \ell}\right)$ is convergent.

(iii) Each column-series is convergent and the iterated series $\sum_{\ell=0}^{\infty}\left(\sum_{k=0}^{\infty} a_{k, \ell}\right)$ is convergent.

In this case,

$$
\sum_{k=0}^{\infty}\left(\sum_{\ell=0}^{\infty} a_{k, \ell}\right)=\sum \sum_{(k, \ell)} a_{k, \ell}=\sum_{\ell=0}^{\infty}\left(\sum_{k=0}^{\infty} a_{k, \ell}\right)
$$

Proof. Suppose ( $i$ ) holds. If $\sum \sum_{(k, \ell)} a_{k, \ell}=s$, then in view of Theorem A.5.1, $\sum_{\ell=1}^{n} a_{k, \ell} \leq$ $s_{k, n} \leq s$ for each fixed $k \in \mathbb{N}_{0}$ and all $n \in \mathbb{N}$. Thus each row-series is a (single) series with non-negative terms whose partial sums are bounded, and hence it is convergent. By 
Fubini's Theorem (Theorem A.3.3), it follows that the iterated series $\sum_{k=0}^{\infty}\left(\sum_{\ell=0}^{\infty} a_{k, \ell}\right)$ is convergent and its sum is equal to $s$.

Suppose (ii) holds. Then for

$$
s_{m, n}=\sum_{k=0}^{m} \sum_{\ell=0}^{n} a_{k \cdot \ell} \leq \sum_{k=0}^{m}\left(\sum_{\ell=0}^{\infty} a_{k \cdot \ell}\right) \leq \sum_{k=0}^{\infty}\left(\sum_{\ell=0}^{\infty} a_{k \cdot \ell}\right)=a .
$$

Therefore, by Theorem A.5.1 the double series $\sum \sum_{(k, \ell)} a_{k, \ell}$ is convergent.

This establishes the equivalence of the statements $(i)$ and (ii). The proof of the equivalence of the statements (i) and (iii) is similar. The equality of the double sum and the sum of either of the two iterated series is also established in this process.

Examples A.3.2 show that the non-negativity of the terms of the double series in Tonelli's Theorem cannot be omitted. The question of the convergence of a double series $\sum \sum_{(k, \ell)} a_{k, \ell}$ with non-negative terms can be reduced to the question of the convergence of each of the following two (single) series, which correspond to summing the double series $\sum \sum_{(k, \ell)} a_{k, \ell}$ "by squares" or "by diagonals" as illustrated in Figure 1.

1. The (single) series $\sum_{j=0}^{\infty} b_{j}$ where for each $j \in \mathbb{N}_{0}, b_{j}$ is the sum of all those terms $a_{k, \ell}$ such that one of $k$ and $\ell$ is equal to $j$ and the other is at most $j$, that is, $b_{j}:=$ $\sum_{i=0}^{j} a_{i, j}+\sum_{i=0}^{j-1} a_{j, i}$. Thus $b_{0}=a_{0,0}, b_{1}=a_{0,1}+a_{1,1}+a_{1,0}, b_{2}=a_{0,2}+a_{1,2}+a_{2,2}+$ $a_{2,0}+a_{2,1}$, and so on.

2. The (single) series $\sum_{j=0}^{\infty} c_{j}$ where for each $j \in \mathbb{N}_{0}, c_{j}$ is the sum of all those terms $a_{k, l}$ such that $k+\ell=j$, that is, $c_{j}:=\sum_{i=0}^{j} a_{j-i, i}$. Thus $c_{0}=a_{0,0}, c_{1}=a_{1,0}+a_{0,1}, c_{2}=$ $a_{2,0}+a_{1,1}+a_{0,2}$, and so on.

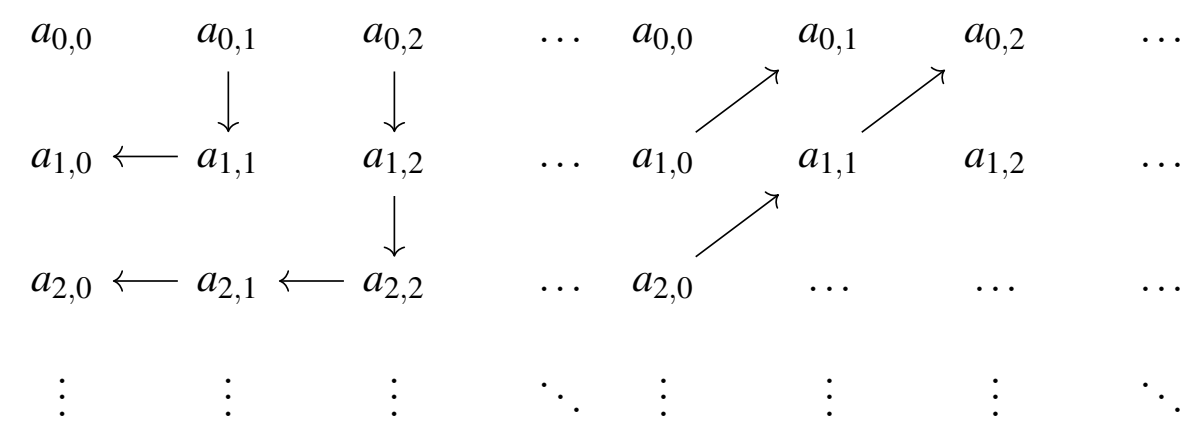

Figure A.1: Summing a double series "by squares" and "by diagonals.” . 
The series $\sum_{j=0}^{\infty} c_{j}$ is sometimes referred to as the diagonal series corresponding to the double series $\sum \sum_{(k, \ell)} a_{k, \ell}$.

Theorem A.5.3. [28]. Let $\sum \sum_{(k, \ell)} a_{k, \ell}$ be a double series with non-negative terms, and $b_{j}, c_{j}$ be as above. Then the following statements are equivalent.

(i) (Summing by Rectangles) $\sum \sum_{(k, \ell)} a_{k, \ell}$ is convergent.

(ii) (Summing by Squares) $\sum_{j=0}^{\infty} b_{j}$ is convergent.

(iii) (Summing by Diagonals) $\sum_{j=0}^{\infty} c_{j}$ is convergent.

In this case,

$$
\sum \sum_{(k, \ell)} a_{k, \ell}=\sum_{j=0}^{\infty} b_{j}=\sum_{j=0}^{\infty} c_{j}
$$

Proof. Let $s_{m, n}:=\sum_{k=0}^{m} \sum_{\ell=0}^{n} a_{k, \ell}$ be the double partial sum of the series $\sum \sum_{(k, \ell)} a_{k, \ell}$, and also let $B_{n}:=\sum_{j=0}^{n} b_{j}$ be the partial sum of the series $\sum_{j=0}^{\infty} b_{j}$. Then by the definition of $b_{j}$, we have $B_{n}=s_{n, n}$ for all $n \in \mathbb{N}_{0}$. Thus, in view of Corollary A.2.1, (i) and (ii) are equivalent, and in this case

$$
\sum \sum_{(k, \ell)} a_{k, \ell}=\sup \left\{s_{m, n}:(m, n) \in \mathbb{N}_{0}^{2}\right\}=\sup \left\{B_{n}=s_{n, n}: n \in \mathbb{N}_{0}\right\}=\sum_{j=0}^{\infty} b_{j}
$$

Next, for $n \in \mathbb{N}_{0}$, let $C_{n}:=\sum_{j=0}^{n} c_{j}$ be the partial sum series of the diagonal series $\sum_{j=0}^{\infty} c_{j}$, where $k+\ell=n$. Then $k \leq n$ and $\ell \leq n$. This implies that $C_{n} \leq s_{n, n}$ for all $n \in \mathbb{N}_{0}$. Also, for any $(k, \ell) \in \mathbb{N}_{0}^{2}$ with $(k, \ell) \preceq(m, n)$ we have $k+\ell \leq m+n=(m+n-1)+1$. This implies that $s_{m, n} \leq C_{m+n-1}$ for all $(m, n) \in \mathbb{N}_{0}^{2}$. In view of these relations, we see that (i) and (iii) are equivalent, and in this case

$$
\sum \sum_{(k, \ell)} a_{k, \ell}=\sup \left\{s_{m, n}:(m, n) \in \mathbb{N}_{0}^{2}\right\}=\sup \left\{C_{n}: n \in \mathbb{N}_{0}\right\}=\sum_{j=0}^{\infty} c_{j} .
$$

Example A.5.1. [28]

(i) Let $p>0$ and for $(k, \ell) \in \mathbb{N}_{0}^{2}$, let $a_{k, l}:=1 /(k+l+1)^{p}$. Then $c_{j}=\sum_{i=0}^{j} 1 /(j+1)^{p}=$ $j /(j+1)^{p}$ for $j \in \mathbb{N}_{0}$. Since

$$
\frac{1}{2(j+1)^{p-1}} \leq \frac{j}{(j+1)^{p}}<\frac{1}{(j+1)^{p-1}} \quad \text { for all } \quad j \in \mathbb{N}_{0} .
$$

The series $\sum_{j=0}^{\infty} c_{j}$ is convergent if and only if $p>2$. So by Theorem A.5.3, we see that $\sum \sum_{(k, \ell)} 1 /(k+\ell+1)^{p}$ is convergent if and only if $p>2$. 
(ii) Since $s_{m, n} \rightarrow s$ as $(m, n) \rightarrow(\infty, \infty)$ implies that $s_{n, n} \rightarrow s$ as $n \rightarrow \infty$, we see that in Theorem A.5.3 the statement (i) implies the statement (ii) irrespective of the sign of the terms of the double series. However, the converse does not hold in general, as Example A.3.2 (ii) shows. In this example, $s_{n, n}=4$ for all $m=n \geq 1$, so that $\sum_{j=0}^{\infty} b_{j}=4$. However, the double series does not converge. This follows by noting that $s_{n, n+1}=3$ for $n \geq 1$.

(iii) Let a double sequence $\left(a_{k, l}\right)$ be schematically given as follows:

$$
\begin{aligned}
& \begin{array}{cccccc}
0 & 1 & 1 & 1 & 1 & \ldots \\
1 & -2 & -1 & -1 & -1 & \ldots
\end{array} \\
& \begin{array}{llllll}
0 & 0 & 0 & 0 & 0 & \ldots
\end{array} \\
& \left(a_{k, \ell}\right)=\begin{array}{cccccc}
1 & -1 & 0 & 0 & 0 & \ldots \\
0 & 0 & 0 & 0 & 0 & \ldots
\end{array} \\
& \begin{array}{llllll}
1 & -1 & 0 & 0 & 0 & \ldots
\end{array} \\
& \vdots \quad \vdots \quad \vdots \quad \vdots \quad \vdots \quad \ddots \\
& \begin{array}{llllllll}
0 & 1 & 2 & 3 & 4 & \ldots & \rightarrow & \infty
\end{array} \\
& \begin{array}{llllllll}
1 & 0 & 0 & 0 & 0 & \ldots & \rightarrow & 0
\end{array} \\
& \begin{array}{llllllll}
1 & 0 & 0 & 0 & 0 & \ldots & \rightarrow & 0
\end{array} \\
& \left(s_{m, n}\right)=\begin{array}{llllllll}
2 & 0 & 0 & 0 & 0 & \ldots & \rightarrow & 0 \\
2 & 0 & 0 & 0 & 0 & \ldots & \rightarrow & 0
\end{array} \\
& \begin{array}{cccccccc}
\vdots & \vdots & \vdots & \vdots & \vdots & \ddots & \downarrow \\
\downarrow & \downarrow & \downarrow & \downarrow & \downarrow & & \searrow & \\
& \downarrow & \downarrow & & &
\end{array} \\
& \infty \quad 0 \quad 0 \quad 0 \quad 0 \quad \rightarrow \quad 0 \quad 0
\end{aligned}
$$

Here $s_{m, n}=0$ for all $m, n \geq 1$ and so $\sum \sum_{(k, \ell)} a_{k, \ell}$ is convergent and its double sum is equal to 0 . However, since $c_{0}=0, c_{1}=2$ and $c_{j}=(-1)^{j}$ for $j \geq 2$, we see that $\sum_{j=0}^{\infty} c_{j}$ is divergent. On the other hand, Example A.3.2 (ii) shows that $\sum \sum_{(k, \ell)} a_{k, \ell}$ may be divergent, while $\sum_{j=0}^{\infty} c_{j}$ is convergent. In this example we note that $c_{0}=2$ and $c_{j}=0 \quad \forall j \geq 1$, so that $\sum_{j=0}^{\infty} c_{j}=2$. It is also possible that both $\sum \sum_{(k, \ell)} a_{k, \ell}$ and $\sum_{j=0}^{\infty} c_{j}$ are convergent but the double sum is not equal to the "sum by diagonals." To illustrate this, consider the double sequence $\left(a_{k, \ell}\right)$ schematically given as follows:

$$
\left(a_{k, \ell}\right)=\begin{array}{cccccc}
1 & 1 & 1 & 1 & 1 & \ldots \\
1 & -1 & -1 & -1 & -1 & \ldots \\
1 & -1 & 0 & 0 & 0 & \ldots \\
1 & -1 & 0 & 0 & 0 & \ldots \\
1 & -1 & 0 & 0 & 0 & \ldots \\
\vdots & \vdots & \vdots & \vdots & \vdots & \ddots
\end{array}
$$




$$
\begin{array}{rccccccc}
1 & 2 & 3 & 4 & 5 & \ldots & \rightarrow & \infty \\
2 & 2 & 2 & 2 & 2 & \ldots & \rightarrow & 2 \\
3 & 2 & 2 & 2 & 2 & \ldots & \rightarrow & 2 \\
4 & 2 & 2 & 2 & 2 & \ldots & \rightarrow & 2 \\
\left(s_{m, n}\right)= & 2 & 2 & 2 & 2 & \ldots & \rightarrow & 2 \\
\vdots & \vdots & \vdots & \vdots & \vdots & \ddots & & \downarrow \\
\downarrow & \downarrow & \downarrow & \downarrow & \downarrow & & \searrow & 2 \\
\infty & 2 & 2 & 2 & 2 & \rightarrow & 2 & 2
\end{array}
$$

Here $s_{m, n}=2$ for $m=n \geq 1$, and so the double sum is equal to 2. But since $c_{0}=1, c_{1}=2, c_{2}=1, c_{j}=0 \quad \forall j \geq 3$, we have $\sum_{j=0}^{\infty} c_{j}=4$.

\section{A.6 Absolute Convergence of Double Series}

This section addresses the convergence and divergence of the double series $\sum \sum_{(k, \ell)}\left|a_{k, \ell}\right|$ formed by considering the absolute values of the terms of a double series $\sum \sum_{(k, \ell)} a_{k, \ell}$. A double series $\sum \sum_{(k, \ell)} a_{k, \ell}$ is said to be absolutely convergent if the double series $\sum \sum_{(k, \ell)}\left|a_{k, \ell}\right|$ is convergent.

Theorem A.6.1. [28]. An absolutely convergent double series is convergent.

Proof. Let $\sum \sum_{(k, \ell)} a_{k, \ell}$ be an absolutely convergent double series. For each $(k, \ell) \in \mathbb{N}_{0}^{2}$, define

$$
a_{k, \ell}^{+}:=\frac{\left|a_{k, \ell}\right|+a_{k, \ell}}{2} \text { and } a_{k, \ell}^{-}:=\frac{\left|a_{k, \ell}\right|-a_{k, \ell}}{2} .
$$

Let $\left(s_{m, n}\right),\left(s_{m, n}^{+}\right),\left(s_{m, n}^{-}\right)$, and $\left(\tilde{s}_{m, n}\right)$ denote the double sequences of $\sum \sum_{(k, \ell)} a_{k, \ell}, \sum \sum_{(k, \ell)} a_{k, \ell}^{+}, \sum \sum_{(k, \ell)} a_{k, \ell}^{-}$, and $\sum \sum_{(k, \ell)}\left|a_{k, \ell}\right|$ respectively. By Theorem A.5.1, $\left(\tilde{s}_{m, n}\right)$ is bounded. Also, $0 \leq a_{k, \ell}^{+} \leq\left|a_{k, \ell}\right|$ and $0 \leq a_{k, \ell}^{-} \leq\left|a_{k, \ell}\right|$ for all $(k, \ell) \in \mathbb{N}_{0}^{2}$, and so

$$
0 \leq s_{k, \ell}^{+} \leq \tilde{s}_{m, n} \quad \text { and } \quad 0 \leq s_{k, \ell}^{-} \leq \tilde{s}_{m, n} \quad \text { for all } \quad(k, \ell) \in \mathbb{N}_{0}^{2},
$$

and therefore, the double sequences $\left(s_{m, n}^{+}\right)$and $\left(s_{m, n}^{-}\right)$are bounded. Using Theorem A.5.1 once again, we see that the double series $\sum \sum_{(k, \ell)} a_{k, \ell}^{+}, \sum \sum_{(k, \ell)} a_{k, \ell}^{-}$are convergent. But $a_{k, \ell}=a_{k, \ell}^{+}-a_{k, \ell}^{-}$for all $(k, \ell) \in \mathbb{N}_{0}^{2}$. Hence, the double series $\sum \sum_{(k, \ell)} a_{k, \ell}$ is convergent.

The concepts of row-series and column-series presented earlier can be used to get the following useful characterization of absolute convergence:

Theorem A.6.2. [28]. A double series $\sum \sum_{(k, \ell)} a_{k, \ell}$ is absolutely convergent if and only if the following conditions hold: 
(i) There are $\left(k_{0}, \ell_{0}\right) \in \mathbb{N}_{0}^{2}$ and $\alpha_{0}>0$ such that

$$
\sum_{k=k_{0}}^{m} \sum_{\ell=\ell_{0}}^{n}\left|a_{k, \ell}\right| \leq \alpha_{0} \quad \forall \quad(m, n) \succeq\left(k_{0}, \ell_{0}\right) .
$$

(ii) Each row-series as well as each column-series is absolutely convergent.

Proof. Suppose $\sum \sum_{(k, \ell)} a_{k, \ell}$ is absolutely convergent. Since $\left|a_{k, \ell}\right| \geq 0$ for all $(k, \ell) \in$ $\mathbb{N}_{0}^{2}$. Theorem A.5.1 shows that condition $(i)$ holds with $\left(k_{0}, l_{0}\right):=(0,0)$, and Tonelli's Theorem for Double Series (Theorem A.5.2) shows that condition (ii) also holds.

Conversely, suppose conditions (i) and (ii) hold. Let $\left(k_{0}, \ell_{0}\right) \in \mathbb{N}_{0}^{2}$ and $\alpha_{0}>0$, we see that for each fixed $k \in \mathbb{N}_{0}$, there is $\beta_{k}>0$ such $\sum_{\ell}\left|a_{k, \ell}\right| \leq \beta_{k}$, and for each fixed $\ell \in \mathbb{N}_{0}$, there is $\gamma_{\ell}>0$ such $\sum_{k}\left|a_{k, \ell}\right| \leq \gamma_{\ell}$. Let $\left(\tilde{s}_{m, n}\right)$ be the double sequence of partial double sums of the double series $\sum \sum_{(k, \ell)}\left|a_{k, \ell}\right|$ and let $p_{0}:=\max \left\{k_{0}, \ell_{0}\right\}$. Then

$$
\begin{aligned}
\tilde{s}_{p, p}=\sum_{k=0}^{p} \sum_{\ell=0}^{p}\left|a_{k, \ell}\right| & =\sum_{k=k_{0}}^{p} \sum_{\ell=\ell_{0}}^{p}\left|a_{k, \ell}\right|+\sum_{k=0}^{p_{0}-1} \sum_{\ell=0}^{p}\left|a_{k, \ell}\right|+\sum_{\ell=0}^{p_{0}-1} \sum_{k=p_{0}}^{p}\left|a_{k, \ell}\right| \\
& \leq \alpha_{0}+\sum_{k=0}^{p_{0}-1} \beta_{k}+\sum_{\ell=0}^{p_{0}-1} \gamma_{\ell} \quad \forall p \in \mathbb{N}_{0} \quad \text { with } \quad p \geq p_{0} .
\end{aligned}
$$

This implies that the diagonal sequence $\left(\tilde{s}_{p, p}\right)$ is bounded, and therefore by Corollary A.2.1, the monotonically increasing double sequence $\left(\tilde{s}_{p, p}\right)$ is bounded. Hence by Theorem A.5.1, $\left(\tilde{s}_{p, p}\right)$ is convergent, which implies that the double series $\sum \sum_{(k, \ell)} a_{k, \ell}$ is absolutely convergent.

Remark A.6.1. Conditions ( $i$ ) and (ii) in Theorem A.6.2 are both needed to characterize absolute convergence. For instance, consider a double sequences $\left(a_{k, l}\right),\left(s_{m, n}\right)$ given schematically as follows:

$$
\begin{array}{rcccccc} 
& 1 & 0 & 0 & 0 & \ldots & \\
& 1 & 0 & 0 & 0 & \ldots & \\
\left(a_{k, \ell}\right)=1 & 0 & 0 & 0 & \ldots & \\
1 & 0 & 0 & 0 & \ldots & \\
& \vdots & \vdots & \vdots & \vdots & \ddots & \\
& & & & & & \\
1 & 1 & 1 & 1 & \ldots & \rightarrow & 1 \\
2 & 2 & 2 & 2 & \ldots & \rightarrow & 2 \\
3 & 3 & 3 & 3 & \ldots & \rightarrow & 3 \\
4 & 4 & 4 & 4 & \ldots & \rightarrow & 4 \\
\vdots & \vdots & \vdots & \vdots & \ddots & & \downarrow \\
\downarrow & \downarrow & \downarrow & \downarrow & & \searrow & \infty \\
\infty & \infty & \infty & \infty & \rightarrow & \infty & \infty
\end{array}
$$


Then condition ( $i$ ) is satisfied with $k_{0}:=0$ and $\ell_{0}:=1$, but $\sum \sum_{(k, \ell)} a_{k, \ell}$ is not (absolutely) convergent, since $s_{m, n}=m+1$ for all $(m, n) \in \mathbb{N}_{0}^{2}$ with $m \geq 1$. On the other hand, if we let $a_{k, \ell}:=1 /(k+\ell+2)^{2}$ for $(k, \ell) \in \mathbb{N}_{0}^{2}$, then condition (ii) is satisfied, but $\sum \sum_{(k, \ell)} a_{k, \ell}$ is not absolutely convergent.

We now show that several results for convergent double series with non- negative terms remain valid for absolutely convergent double series.

Theorem A.6.3. [28]. Let $\sum \sum_{(k, \ell)} a_{k, \ell}$ be an absolutely convergent double series. Then the following hold.

(i) The double sequence $\left(s_{m, n}\right)$ of partial double sums is bounded.

(ii) Each row-series as well as each column-series is absolutely convergent, and

$$
\sum_{k=0}^{\infty}\left(\sum_{\ell=0}^{\infty} a_{k, \ell}\right)=\sum \sum_{(k, \ell)} a_{k, \ell}=\sum_{\ell=0}^{\infty}\left(\sum_{k=0}^{\infty} a_{k, \ell}\right)
$$

(iii) The corresponding diagonal series $\sum_{j=0}^{\infty} c_{j}$ is absolutely convergent, and

$$
\sum_{j=0}^{\infty} c_{j}=\sum \sum_{(k, \ell)} a_{k, \ell}
$$

Proof. For $(m, n) \in \mathbb{N}_{0}^{2}$, let $s_{m, n}$ and $\tilde{s}_{m, n}$ denote the $(m, n) t h$ partial double sums of $\sum \sum_{(k, \ell)} a_{k, \ell}$ and $\sum \sum_{(k, \ell)}\left|a_{k, \ell}\right|$ and let $s$ and $\tilde{s}$ denote their double sums, respectively.

Now (i) follows from Theorem A.5.1, since $\left|s_{m, n}\right| \leq \tilde{s}_{m, n}$ for all $(m, n) \in \mathbb{N}_{0}^{2}$, while (ii) follows from Theorem A.6.2 and Fubini's Theorem (Theorem A.3.3).

To prove (iii), let $\sum_{1}:=\sum_{j=0}^{\infty} c_{j}$ and $\sum_{2}:=\sum_{j=0}^{\infty} d_{j}$ denote the diagonal series corresponding to the double series $\sum \sum_{(k, \ell)} a_{k, \ell}$ and $\sum \sum_{(k, \ell)}\left|a_{k, \ell}\right|$ respectively, and for $n \in \mathbb{N}_{0}$, let $C_{n}$ and $D_{n}$ denote the corresponding $n t h$ partial sums of the series $\sum_{1}$ and $\sum_{2}$ respectively. By Theorem A.5.3, it follows that $D_{n} \rightarrow \tilde{s}$. But since $\left|c_{j}\right| \leq d_{j}$ for all $j \in \mathbb{N}_{0}$ and the sequence $\left(D_{n}\right)$ is bounded, we see that the sequence $\left(\sum_{j=0}^{\infty} c_{j}\right)$ is bounded, and so the series $\sum_{j=0}^{\infty} c_{j}$ converges absolutely. Now it can be easily seen that $\left|s_{n, n}-C_{n}\right| \leq\left|\tilde{s}_{n, n}-D_{n}\right|$ for all $n \in \mathbb{N}_{0}$. Since $\tilde{s}_{n, n} \rightarrow \tilde{s}$ and also $D_{n} \rightarrow \tilde{s}$, we see that the sequences $\left(s_{n, n}\right)$ and $\left(C_{n}\right)$ have the same limit, that is, $\sum_{j=0}^{\infty} c_{j}=\sum \sum_{(k, \ell)} a_{k, \ell}$ as desired.

Example A.3.2 (ii) shows that a double series may diverge even if the corresponding diagonal series is absolutely convergent. 


\section{A.7 Convergence Tests for Double Series}

The following results provide techniques to test weather double series converge absolutely or not.

Theorem A.7.1. (Comparison Test for Double Series)[28]. Let $a_{k, \ell}$ be complex numbers and $b_{k, \ell}$ real numbers such that $\left|a_{k, l}\right| \leq b_{k, \ell}$ for all $(k . \ell) \in \mathbb{N}_{0}^{2}$. If

$\sum \sum_{(k, \ell)} b_{(k, \ell)}$ is convergent, then $\sum \sum_{(k, \ell)} a_{(k, \ell)}$ is absolutely convergent and

$$
\left|\sum_{(k, \ell)} \sum_{k, \ell}\right| \leq \sum \sum_{(k, \ell)} b_{k, \ell}
$$

Proof. Suppose $\sum \sum_{(k, \ell)} b_{k, \ell}$ is convergent. For $(m, n) \in \mathbb{N}_{0}^{2}$, we have

$$
\left|\sum_{k=1}^{m} \sum_{\ell=1}^{n} a_{k, \ell}\right| \leq \sum_{k=1}^{m} \sum_{\ell=1}^{n}\left|a_{k, \ell}\right| \leq \sum_{k=1}^{m} \sum_{\ell=1}^{n} b_{k, \ell} .
$$

Since $b_{k, \ell} \geq 0$ for all $(m, n) \in \mathbb{N}_{0}^{2}$, the double sequence of the partial double partial sum of $\sum \sum_{(k, \ell)} b_{k, \ell}$ is bounded above (Theorem A.5.1). By Inequalities (A.6) the same holds for $\sum \sum_{(k, \ell)}\left|a_{k, \ell}\right|$. Also, since $\left|a_{k, \ell}\right| \geq 0$ for all $(m, n) \in \mathbb{N}_{0}^{2}$, it follows from Theorem A.5.1 that $\sum \sum_{(k, \ell)}\left|a_{k, \ell}\right|$ is convergent, that is $\sum \sum_{(k, \ell)} a_{k, \ell}$ is absolutely convergent. Inequalities (A.6) also imply that $\left|\sum \sum_{(k, \ell)} a_{k, \ell}\right| \leq \sum \sum_{(k, \ell)}\left|a_{k, \ell}\right| \leq \sum \sum_{(k, \ell)} b_{k, \ell}$.

We will now consider analogues of the limit comparison test, the root test, and the ratio test for double series. We first state some basic results in the case of a (single) series for ease of reference.

Fact A.7.1. [28] Let $\left(a_{k}\right)$ be a sequence of real numbers.

(i) Assume that $a_{k}>0$ for all $k \in \mathbb{N}_{0}$. Let $\left(b_{k}\right)$ be a sequence of positive real numbers such that $a_{k} / b_{k} \rightarrow r$ as $k \rightarrow \infty$, where $r \in \mathbb{N}_{0}$ with $r \neq 0$. Then the series $\sum_{k} a_{k}$ is convergent if and only if the series $\sum_{k} b_{k}$ is convergent.

(ii) If there is $\alpha \in \mathbb{R}$ with $\alpha<1$ such that $\left|a_{k}\right|^{1 / k} \leq \alpha$ for all large $k$, then the series $\sum_{k} a_{k}$ is absolutely convergent. If $\left|a_{k}\right|^{1 / k} \geq 1$ for infinitely many $k \in \mathbb{N}_{0}$, then the series $\sum_{k} a_{k}$ is divergent.

(iii) If there is $\alpha \in \mathbb{R}$ with $\alpha<1$ such that $\left|a_{k+1}\right| \leq \alpha\left|a_{k}\right|$ for all large $k$, then the series $\sum_{k} a_{k}$ is absolutely convergent. If $\left|a_{k+1}\right| \geq\left|a_{k}\right|>0$ for all large $k \in \mathbb{N}$, then the series $\sum_{k} a_{k}$ is divergent.

The result below leads to the limit comparison test for double series, which is often easier to use than the comparison test. 
Theorem A.7.2. [28]. Let $\left(a_{k, \ell}\right)$ and $\left(b_{k, \ell}\right)$ be double sequences such that $b_{k, \ell} \neq 0$ for all $(k, \ell) \in \mathbb{N}_{0}^{2}$. Suppose each row-series as well as each column-series corresponding to both $\sum \sum_{(k, \ell)} a_{k, \ell}$ and $\sum \sum_{(k, \ell)} b_{k, \ell}$ is absolutely convergent, and $a_{k, \ell} / b_{k, \ell} \rightarrow \operatorname{ras}(k, \ell) \rightarrow(\infty, \infty)$, where $r \in \mathbb{R} \cup\{ \pm \infty\}$.

(i) If $b_{k, \ell}>0$ for all $(k, \ell) \in \mathbb{N}_{0}^{2}, \sum \sum_{(k, \ell)} b_{k, \ell}$ is convergent, and $r \in \mathbb{R}$, then $\sum \sum_{(k, \ell)} a_{k, \ell}$ is absolutely convergent.

(ii) If $a_{k, \ell}>0$ for all $(k, \ell) \in \mathbb{N}_{0}^{2}, \sum \sum_{(k, \ell)} a_{k, \ell}$ is convergent, and $r \neq 0$, then $\sum \sum_{(k, \ell)} b_{k, \ell}$ is absolutely convergent.

\section{Proof.}

(i) Suppose $b_{k, \ell}>0$ for all $(k, \ell) \in \mathbb{N}_{0}^{2}$, and the double series $\sum \sum_{(k, \ell)} b_{k, \ell}$ is convergent. Let $r \in \mathbb{R}$ such that $a_{k, \ell} / a_{k, \ell} \rightarrow r$ as $(k, \ell) \rightarrow(\infty, \infty)$, then there is $\left(k_{0}, \ell_{0}\right) \in \mathbb{N}_{0}^{2}$ such that for all $(k, \ell) \succeq\left(k_{0}, \ell_{0}\right)$

$$
(r-1) b_{k, \ell}<a_{k, \ell}<(r+1) b_{k, \ell} \quad \text { and so } \quad\left|a_{k, \ell}\right|<\max \{|r-1|,|r+1|\} b_{k, \ell} .
$$

Also, by Theorem A.5.1 there is $\beta>0$ such that $\sum_{k=1}^{m} \sum_{\ell=1}^{n} a_{k, \ell} \leq \beta$ for $(m, n) \in \mathbb{N}_{0}^{2}$. Hence for all $(m, n) \succeq\left(k_{0}, \ell_{0}\right)$, we have

$$
\sum_{k=k_{0}}^{m} \sum_{\ell=\ell_{0}}^{n}\left|a_{k, \ell}\right| \leq \max \{|r-1|,|r+1|\} \sum_{k=k_{0}}^{m} \sum_{\ell=\ell_{0}}^{n} b_{k, \ell} \leq \max \{|r-1|,|r+1|\} \beta .
$$

By Theorem A.6.2, the double series $\sum \sum_{(k, \ell)} a_{k, \ell}$ is absolutely convergent.

(ii) Suppose $a_{k, \ell}>0$ for all $(k, \ell) \in \mathbb{N}_{0}^{2}$ and $r \neq 0$. Then the limit of $b_{k, \ell} / a_{k, \ell}$ as $(k, \ell) \rightarrow(\infty, \infty)$ is $1 / r$ or 0 according as $r \in \mathbb{R}$ or $r=\infty$. By interchanging $a_{k, \ell}$ and $b_{k, \ell}$ in $(i)$ above, the desired result follows.

Corollary A.7.1. (Limit Comparison Test for Double Series)[39]. Let $\left(a_{k, \ell}\right)$ and $\left(b_{k, \ell}\right)$ be double sequences of positive real numbers. Suppose each row-series as well as each column-series corresponding to both $\sum \sum_{(k, \ell)} a_{k, \ell}$ and $\sum \sum_{(k, \ell)} b_{k, \ell}$ are convergent, and

$$
\lim _{(k, \ell) \rightarrow(\infty, \infty)} \frac{a_{k, \ell}}{a_{k, \ell}}=r \quad \text { where } \quad r \in \mathbb{R} \quad \text { with } \quad r \neq 0 .
$$

Then

$$
\sum \sum_{(k, \ell)} a_{k, \ell} \text { is convergent } \Longleftrightarrow \sum \sum_{(k, \ell)} b_{k, \ell} \text { is convergent }
$$


Remark A.7.1. [39]. In the Limit Comparison Test for Double Series, it is not possible to drop the condition $r \in \mathbb{R}$ and $r \neq 0$. To see that $r=0$ will not work, let

$$
a_{k, \ell}:=\frac{1}{(k+1)^{2}(\ell+1)^{2}} \quad \text { and } \quad b_{k, \ell}:=\frac{1}{(k+\ell+2)^{2}} \quad \text { for } \quad(k, \ell) \in \mathbb{N}_{0}^{2} .
$$

Then $\lim _{\ell \rightarrow \infty} a_{k, \ell} / b_{k, \ell}=1 /(k+1)^{2}$ for each $k \in \mathbb{N}_{0}$, and $\lim _{k \rightarrow \infty} a_{k, \ell} / b_{k, \ell}=1 /(\ell+1)^{2}$ for each $\ell \in \mathbb{N}_{0}$. Hence by Fact A.7.1 (i), we see that each row-series as well as each column-series corresponding to both $\sum \sum_{(k, \ell)} a_{k, \ell}$ and $\sum \sum_{(k, \ell)} b_{k, \ell}$ is convergent. However, $\lim _{(k, \ell) \rightarrow(\infty, \infty)} a_{k, \ell} / b_{k, \ell}=0$, and as shown in Examples A.3.1 (iii), the double series $\sum \sum_{(k, \ell)} a_{k, \ell}$ converges, while the double series $\sum \sum_{(k, \ell)} b_{k, \ell}$ diverges. By interchanging the definitions of $a_{k, \ell}$ and $b_{k, \ell}$, we see that $r=\infty$ will also not work.

Example A.7.1. [39].

(i) Let $a_{k, \ell}:=\sin \left(1 /\left[(k+1)^{2}(\ell+1)^{2}\right]\right)$ for all $(k, \ell) \in \mathbb{N}_{0}^{2}$. Consider $b_{k, \ell}:=1 /[(k+$ $\left.1)^{2}(\ell+1)^{2}\right]$ for all $(k, \ell) \in \mathbb{N}_{0}^{2}$, and observe that $\left(a_{k, \ell} / a_{k, \ell}\right) \rightarrow 1$ as $(k, \ell) \rightarrow(\infty, \infty)$. Since $\sum \sum_{(k, \ell)} b_{k, \ell}$ is convergent. Corollary A.7.1 shows that $\sum \sum_{(k, \ell)} a_{k, \ell}$ is convergent.

(ii) Let $a_{k, \ell}:=\sin \left(1 /(k+\ell+2)^{2}\right)$ for all $(k, \ell) \in \mathbb{N}_{0}^{2}$. Consider $b_{k, \ell}:=1 /(k+\ell+$ $2)^{2}$ for all $(k, \ell) \in \mathbb{N}_{0}^{2}$, and observe that $\left(a_{k, \ell} / a_{k, \ell}\right) \rightarrow 1$ as $(k, \ell) \rightarrow(\infty, \infty)$. Since $\sum \sum_{(k, \ell)} b_{k, \ell}$ is divergent. Corollary A.7.1 now shows that $\sum \sum_{(k, \ell)} a_{k, \ell}$ is divergent.

The following result will lead us to Cauchy's root test, or simply the root test, which is one of the most basic tests to determine the absolute convergence of a double series:

For the rest of this section, we will say that a statement holds whenever "both $k$ and $\ell$ are large" to mean that there is $\left(k_{0}, \ell_{0}\right) \in \mathbb{N}_{0}^{2}$ such that the statement holds for all $(k, \ell) \in$ $\mathbb{N}_{0}^{2} \backslash\{(0,0)\}$ with $(k, \ell) \succeq\left(k_{0}, \ell_{0}\right)$.

Theorem A.7.3. [39]. Let $\left(a_{k, l}\right)$ be a double sequence of complex numbers.

(i) Suppose each row-series as well as each column-series corresponding to $\sum_{(k, \ell)} a_{k, \ell}$ is absolutely convergent. If there is $\alpha \in \mathbb{R}$ with $\alpha<1$ such that $\left|a_{k, \ell}\right|^{1 / k+\ell} \leq \alpha$ whenever both $k$ and $l$ are large, then $\sum \sum_{(k, \ell)} a_{k, \ell}$ is absolutely convergent.

(ii) Iffor each $\left(k_{0}, \ell_{0}\right) \in \mathbb{N}_{0}^{2} \backslash\{(0,0)\}$, there is $(k, \ell) \in \mathbb{N}_{0}^{2}$ such that $(k, \ell) \succeq\left(k_{0}, \ell_{0}\right)$ and $\left|a_{k, \ell}\right|^{1 / k+\ell} \geq 1$, then $\sum \sum_{(k, \ell)} a_{k, \ell}$ is divergent.

Proof. 
(i) Suppose there are $\alpha \in \mathbb{R}$ with $\alpha<1$ and $\left(k_{0}, \ell_{0}\right) \in \mathbb{N}_{0}^{2} \backslash\{(0,0)\}$ such that $\left|a_{k, \ell}\right|^{1 / k+\ell} \leq$ $\alpha$ for all $(k, \ell) \succeq\left(k_{0}, \ell_{0}\right)$. Then $\alpha \geq 0$ and

$$
\sum_{k=k_{0}}^{m} \sum_{\ell=\ell_{0}}^{n}\left|a_{k, \ell}\right| \leq\left(\sum_{k=k_{0}}^{m} \alpha^{k}\right)\left(\sum_{\ell=\ell_{0}}^{n} \alpha^{\ell}\right)=\frac{1}{(1-\alpha)^{2}} \quad \text { for all } \quad(m, n) \succeq\left(k_{0}, \ell_{0}\right) .
$$

Therefore, $(i)$ follows from Theorem A.6.2.

(ii) Suppose for each $\left(k_{0}, \ell_{0}\right) \in \mathbb{N}_{0}^{2} \backslash\{(0,0)\}$, there is $(k, \ell) \in \mathbb{N}_{0}^{2}$ such that $(k, \ell) \succeq\left(k_{0}, \ell_{0}\right)$ and $\left|a_{k, \ell}\right|^{1 / k+\ell} \geq 1$, that is, $\left|a_{k, \ell}\right| \geq 1$. Hence $a_{k, l} \nrightarrow 0$ as $(k, l) \rightarrow(\infty, \infty)$. By the $(k, \ell)$ th Term Test (Theorem A.3.1), it follows that $\sum \sum_{(k, \ell)} a_{k, \ell}$ is divergent.

Corollary A.7.2. (Root Test for Double Series)[39]. Let $\left(a_{k, l}\right)$ be a double sequence of complex numbers such that $\left|a_{k, \ell}\right|^{1 / k+\ell} \rightarrow$ a as $(k, \ell) \rightarrow(\infty, \infty)$, where $a \in \mathbb{R} \cup\{\infty\}$. If each row-series as well as each column-series corresponding to $\sum \sum_{(k, \ell)} a_{k, \ell}$ is absolutely convergent and $a<1$, then $\sum \sum_{(k, \ell)} a_{k, \ell}$ is absolutely convergent. On the other hand, if $a>1$, then $\sum \sum_{(k, \ell)} a_{k, \ell}$ is divergent, and all but finitely many row-series and columnseries are also divergent.

Proof. The first assertion follows from part (i) of Theorem A.7.3 with $\alpha:=(1+a) / 2$. Now suppose $a>1$. Then there is $\left(k_{0}, \ell_{0}\right) \in \mathbb{N}_{0}^{2}$ such that $\left|a_{k, \ell}\right|^{1 / k+\ell} \geq 1$ for all $(k, \ell) \succeq$ $\left(k_{0}, \ell_{0}\right)$. Part (ii) of Theorem A.7.3 shows that $\sum \sum_{(k, \ell)} a_{k, \ell}$ is divergent. Also, for each fixed $k \geq k_{0}$, we see that $a_{k, \ell} \nrightarrow 0$ as $\ell \rightarrow \infty$ and hence the row-series $\sum_{\ell} a_{k, \ell}$ is diverges. Similarly, for each fixed $\ell \geq \ell_{0}$, the column-series $\sum_{k} a_{k, \ell}$ diverges.

The following result will lead us to D'Alembert's ratio test, or simply the ratio test, which is another basic test to determine the absolute convergence of a double series:

Theorem A.7.4. [28]. Let $a_{k, \ell}$ be a double sequence of complex numbers.

(i) Suppose each row-series as well as each column-series corresponding to the double series $\sum \sum_{(k, \ell)} a_{k, \ell}$ is absolutely convergent. If there is $\alpha \in \mathbb{R}$ with $\alpha<1$ such that either $\left|a_{k, \ell+1}\right| \leq \alpha\left|a_{k, l}\right|$ whenever both $k$ and $\ell$ are large, or $\left|a_{k+1, \ell}\right| \leq \alpha\left|a_{k, l}\right|$ whenever both $k$ and $\ell$ are large, then $\sum \sum_{(k, \ell)} a_{k, \ell}$ is absolutely convergent.

(ii) If $\min \left\{\left|a_{k, \ell+1}\right|,\left|a_{k+1, \ell}\right|\right\} \geq\left|a_{k, \ell}\right|>0$ whenever both $k$ and lare large, then $\sum \sum_{(k, \ell)} a_{k, \ell}$ is divergent, and all but finitely many row-series and column-series is also divergent.

\section{Proof.}

(i) Suppose there are $\alpha \in \mathbb{R}$ with $\alpha<1$ and $\left(k_{0}, \ell_{0}\right) \in \mathbb{N}_{0}^{2}$ such that $\left|a_{k, \ell+1}\right| \leq \alpha\left|a_{k, \ell}\right|$ for all $(k, \ell) \succeq\left(k_{0}, \ell_{0}\right)$. We may assume that $\alpha>0$. Now

$$
\left|a_{k, \ell}\right| \leq \alpha\left|a_{k, \ell-1}\right| \leq \cdots \leq \alpha^{\ell-\ell_{0}}\left|a_{k, \ell_{0}}\right| \quad \text { for all } \quad(k, \ell) \succeq\left(k_{0}, \ell_{0}\right) .
$$


Since $0<\alpha<1$, we see that $\sum_{\ell=0}^{n} \alpha^{n} \leq \frac{1}{1-\alpha}$ for all $n \in \mathbb{N}_{0}$. Also, the series $\sum_{k} a_{k, \ell_{0}}$ is absolutely convergent, there is $\beta>0$ such that $\sum_{k=1}^{m}\left|a_{k, \ell_{0}}\right| \leq \beta$, for all $m \in \mathbb{N}_{0}$. Consequently,

$$
\sum_{k=k_{0}}^{m} \sum_{\ell=\ell_{0}}^{n}\left|a_{k, \ell}\right| \leq \frac{\alpha^{-\ell_{0}} \beta}{1-\alpha} \quad \text { for all } \quad(m, n) \succeq\left(k_{0}, \ell_{0}\right) .
$$

Hence by Theorem A.6.2, $\sum \sum_{(k, \ell)} a_{k, \ell}$ is absolutely convergent. A similar argument holds if there is $\alpha \in \mathbb{R}$ with $\alpha<1$ and $\left(k_{0}, \ell_{0}\right) \in \mathbb{N}_{0}^{2}$ such that $\left|a_{k+1, \ell}\right| \leq \alpha\left|a_{k, \ell}\right|$ for all $(k, \ell) \succeq\left(k_{0}, \ell_{0}\right)$.

(ii) Suppose there is $\left(k_{0}, \ell_{0}\right) \in \mathbb{N}_{0}^{2}$ such that $\min \left\{\left|a_{k, \ell+1}\right|,\left|a_{k+1, \ell}\right|\right\} \geq\left|a_{k, \ell}\right|>0$ for all $(k, \ell) \succeq\left(k_{0}, \ell_{0}\right)$. Then,

$$
\left|a_{k, \ell}\right| \geq\left|a_{k, \ell-1}\right| \geq \cdots \geq\left|a_{k, \ell_{0}}\right| \geq\left|a_{k-1, \ell_{0}}\right| \geq \cdots \geq\left|a_{k_{0}, \ell_{0}}\right|>0
$$

for all $(k, \ell) \succeq\left(k_{0}, \ell_{0}\right)$. Since $a_{k_{0}, \ell_{0}} \neq 0$, we see that $a_{k, \ell} \nrightarrow 0$ as $(k, \ell) \rightarrow(\infty, \infty)$, and further, and further, for each fixed $k \geq k_{0}, a_{k, \ell} \nrightarrow 0$ as $\ell \rightarrow \infty$ and for each fixed $\ell \geq \ell_{0}$, $a_{k, \ell} \nrightarrow 0$ as $k \rightarrow \infty$. The desired results now follow from the $(k, \ell) t h$ Term Test for double series (Theorem A.3.1) and the $k$ th Term Test for (single) series.

Corollary A.7.3. [28]. (Ratio Test for Double Series). Let $\left(a_{k, l}\right)$ be a double sequence of nonzero real numbers such that either $\left|a_{k, \ell+1}\right| /\left|a_{k, \ell}\right| \rightarrow a$ or

$\left|a_{k+1}, \ell\right| /\left|a_{k, l}\right| \rightarrow \tilde{a}$ as $(k, \ell) \rightarrow(\infty, \infty)$, where $a, \tilde{a} \in \mathbb{R} \cup\{\infty\}$. If each row-series as well as each column-series corresponding to $\sum \sum_{(k, \ell)} a_{k, \ell}$ is absolutely convergent and $a<1$ or $\tilde{a}<1$, then is $\sum \sum_{(k, \ell)} a_{k, \ell}$ absolutely convergent. On the other hand, if $a>1$, then $\sum \sum_{(k, \ell)} a_{k, \ell}$ is divergent and all but finitely many row-series are also divergent, while if $\tilde{a}>1$, then $\sum \sum_{(k, \ell)} a_{k, \ell}$ is divergent and all but finitely many column-series are also divergent.

Proof. The first result is a consequence of part ( $i$ ) of Theorem A.7.4 with $\alpha:=(1+a) / 2$ or $\alpha:=(1+\tilde{a}) / 2$ according as $a<1$ or $\tilde{a}<1$.

Now suppose $a>1$. Then there is $\alpha \in \mathbb{R}$ with $\alpha>1$ and $\left(k_{0}, \ell_{0}\right) \in \mathbb{N}_{0}^{2}$ such that $\left|a_{k, \ell+1}\right| /\left|a_{k, l}\right| \geq \alpha$ for all $(k, \ell) \succeq\left(k_{0}, \ell_{0}\right)$. Then

$$
\left|a_{k, \ell+1}\right| /\left|a_{k, \ell}\right| \geq \alpha \quad \text { for all }(k, \ell) \succeq\left(k_{0}, \ell_{0}\right) .
$$

Given any $\left(k_{1}, \ell_{1}\right) \in \mathbb{N}_{0}^{2}$, let $k:=\max \left\{k_{0}, k_{1}\right\}$. Since $\alpha>1$ and $a_{k, \ell_{0}} \neq 0$, we can find $\ell \geq \max \left\{\ell_{0}, \ell_{1}\right\}$ such that $\alpha^{\ell-\ell_{0}}\left|a_{k, \ell_{0}}\right| \geq 1$. Then $k \geq k_{1}, \ell \geq \ell_{1}$, and $\left|a_{k, \ell}\right| \geq 1$. This shows that $a_{k, \ell} \neq 0$ as $(k, \ell) \rightarrow(\infty, \infty)$, and so $\sum \sum_{(k, \ell)} a_{k, \ell}$ is divergent by the $(k, \ell)$ th Term Test. Also, for each fixed $k \geq k_{0}$, we have $\left|a_{k, \ell}\right| \geq \alpha^{\ell-\ell_{0}}\left|a_{k, \ell_{0}}\right| \geq\left|a_{k, \ell_{0}}\right|>0$ for all $\ell \geq \ell_{0}$, and so $a_{k, \ell} \nrightarrow 0$ as $\ell \rightarrow \infty$, which implies that $\sum_{\ell} a_{k, \ell}$ is divergent. Similar arguments hold if $\tilde{a}>1$. 
Example A.7.2. [28]

(i) If the limit a in the Root Test (Corollary A.7.2) is equal to 1, then the double series $\sum \sum_{(k, \ell)} a_{k, \ell}$ may converge absolutely or it may diverge. The same holds if the limits $a$ and $\tilde{a}$ in the Ratio Test (Corollary A.7.3) are equal to 1. For example, let $a_{k, \ell}:=$ $1 /(k+1)^{2}(\ell+1)^{2}$ and $b_{k, \ell}: 1 /(k+\ell+2)^{2}$ for all $(k, \ell) \in \mathbb{N}_{0}^{2}$. Then it is easy to see that each row-series as well as each column-series corresponding to both $\sum \sum_{(k, \ell)} a_{k, \ell}$ and $\sum \sum_{(k, \ell)} b_{k, \ell}$ is (absolutely) convergent and all the above-mentioned limits are equal to 1 for both cases. However, as we have seen in Example A.3.1 (iii) $\sum \sum_{(k, \ell)} a_{k, \ell}$ is (absolutely) convergent, but $\sum \sum_{(k, \ell)} b_{k, \ell}$ is divergent.

(ii) Let $p>0$ and for $(k, \ell) \in \mathbb{N}_{0}^{2}$, let $a_{k, \ell}:=(k+\ell)^{p} / 2^{k} 3^{\ell}$. It easy to see (using Fact A.7.1 (iii), for example) that each row-series as well as each column-series corresponding to $\sum \sum_{(k, \ell)} a_{k, \ell}$ is (absolutely) convergent. Since, $\left|a_{k, \ell+1}\right| /\left|a_{k, \ell}\right| \rightarrow \frac{1}{3}$ as $(k, \ell) \rightarrow(\infty, \infty)$, Corollary A.7.3 shows that $\sum \sum_{(k, \ell)} a_{k, \ell}$ is (absolutely) convergent. Alternatively, the same conclusion follows by noting that $\left|a_{k+1, \ell}\right| /\left|a_{k, \ell}\right| \rightarrow \frac{1}{2}$ as $(k, \ell) \rightarrow(\infty, \infty)$.

(iii) For $(k, \ell) \in \mathbb{N}_{0}^{2}$, let $a_{k, \ell}:=(k+\ell) ! / 2^{k} 3^{\ell}$. Since $a_{k, \ell+1} / a_{k, \ell} \rightarrow \infty$ as $(k, \ell) \rightarrow(\infty, \infty)$, Corollary A.7.3 shows that $\sum \sum_{(k, \ell)} a_{k, \ell}$ is divergent. Alternatively, observe that $a_{k, \ell} \geq\left(k ! / 2^{k}\right)\left(\ell ! / 3^{\ell}\right) \geq 1$ for $(k, \ell) \succeq(4,7)$, and so the $(k, \ell)$-th Term Test shows that $\sum \sum_{(k, \ell)} a_{k, \ell}$ is divergent.

(iv) $\operatorname{For}(k, \ell) \in \mathbb{N}_{0}^{2}$, let $a_{k, \ell}:=(k+\ell+2) ! /(k+\ell+2)^{k+\ell+2}$. Since $(1+(1 / n))^{n} \rightarrow$ e as $n \rightarrow \infty$, where $e$ is the base of the natural logarithm, we see that $a_{k, \ell+1} / a_{k, \ell} \rightarrow 1 / e$ as $(k, \ell) \rightarrow(\infty, \infty)$. Also, for each fixed $k \in \mathbb{N}_{0}$, we have $\lim _{\ell \rightarrow \infty} a_{k, \ell+1} / a_{k, \ell}=1 / e$, and for each fixed $\ell \in \mathbb{N}_{0}$, we have $\lim _{\ell \rightarrow \infty} a_{k+1, \ell} / a_{k, \ell}=1 /$ e. Since $e>1$, Corollary A.7.3 and Fact A.7.1 (iii) show that $\sum \sum_{(k, \ell)} a_{k, \ell}$ is (absolutely) convergent.

(v) $\operatorname{For}(k, \ell) \in \mathbb{N}_{0}^{2}$, let

$$
a_{k, \ell}:=\left\{\begin{array}{llll}
\frac{1}{2^{k+\ell+2}} & \text { if } & k+\ell & \text { is even } \\
\frac{1}{3^{k+\ell+2}} & \text { if } & k+\ell & \text { is odd }
\end{array}\right.
$$

Since $\left|a_{k, \ell+1}\right| /\left|a_{k, l}\right|=\left|a_{k+1, \ell}\right| /\left|a_{k, l}\right|=2^{k+\ell+2} / 3^{k+\ell+3} \leq 4 / 27$ if $k+\ell$ is even, and $\left|a_{k, \ell+1}\right| /\left|a_{k, l}\right|=\left|a_{k+1, \ell}\right| /\left|a_{k, l}\right|=3^{k+\ell+2} / 2^{k+\ell+3} \geq 27 / 16$ is odd, the Ratio test for Double Series (Corollary A.7.3) is not applicable to this example. For the same reason, Theorem A.7.4 is also not applicable. Further, since the double sequence $\left(\left|a_{k, \ell}\right|^{1 /(k+\ell)}\right)$ does not converge, the Root Test for Double Series (Corollary A.7.2) is not applicable. However, since $\left|a_{k, \ell}\right|^{1 / \ell}$ and $\left|a_{k, \ell}\right|^{1 / k}$ are less than or equal to $1 / 2$ for all $(k, \ell) \in \mathbb{N}_{0}^{2}$, we see that each row-series as well as each column-series 
corresponding to $\sum \sum_{(k, \ell)} a_{k, \ell}$ is (absolutely) convergent. Also, $\left|a_{k, \ell}\right|^{1 / k+\ell} \leq 1 / 2<1$ for all $(k, \ell) \in \mathbb{N}_{0}^{2}$, and hence Theorem A.7.3 is applicable. Thus $\sum \sum_{(k, \ell)} a_{k, \ell}$ is (absolutely) convergent.

\section{A.8 Double Power Series}

For $k, \ell \in \mathbb{N}_{0}$, let $c_{k, \ell} \in \mathbb{R}$. The double series

$$
\sum_{\substack{p=0 \\ k+\ell=0}} c_{k, \ell} x^{k} y^{\ell}=\sum \sum_{(k, \ell) \succeq(0,0)} c_{k, \ell} x^{k} y^{\ell}
$$

is called a double power series (around $(0,0)$ ), and for $(k, \ell) \succeq(0,0)$, the real number $c_{k, \ell}$ is called its $(k, \ell)$ th coefficient. Henceforth when we consider a double power series

$\sum_{\sum} c_{k, \ell} x^{k} y^{\ell}$, it will be tacitly assumed that the index $(k, \ell)$ varies over the set of all pairs of $\mathbb{N}_{0}^{2}$. For $(m, n) \succeq(0,0)$, the $(m, n)$ th partial double sum of the double power series $\sum \sum_{(k, \ell)} c_{k, \ell} x^{k} y^{\ell}$ is

$$
A_{m, n}(x, y):=\sum_{k=0}^{m} \sum_{\ell=0}^{n} c_{k, \ell} x^{k} y^{\ell} .
$$

It is clear that if $(x, y)=(0,0)$, then for any choice of the coefficients $c_{k, \ell}$, the double power series $\sum \sum_{(k, \ell)} c_{k, \ell} x^{k} y^{\ell}$ is convergent and its double sum is equal to $c_{0,0}$. Also, if $x \in \mathbb{R}$ and $y=0$, then the double power series is convergent if and only if the (single) power series $\sum_{k=0}^{\infty} c_{k, 0} x^{k}$ is convergent, and likewise, if $x=0$ and $y \in \mathbb{R}$, then the double power series is convergent if and only if the (single) power series $\sum_{\ell=0}^{\infty} c_{0, \ell} y^{\ell}$ is convergent. On the other hand, if there is $\left(k_{0}, \ell_{0}\right) \in \mathbb{N}_{0}^{2}$ such that $c_{k, \ell}=0$ whenever either $k>k_{0}$ or $\ell>\ell_{0}$, then the double power series is convergent for any $(x, y) \in \mathbb{R}^{2}$, and its double sum is equal to

$$
\sum_{k=0}^{k_{0}} \sum_{\ell=0}^{\ell_{0}} c_{k, \ell} x^{k} y^{\ell}
$$

More generally, if $\left(x_{0}, y_{0}\right) \in \mathbb{R}^{2}$, then the double series

$$
\sum_{(k, \ell) \succeq(0,0)}\left(x-x_{0}\right)^{k}\left(y-y_{0}\right)^{\ell}
$$

is called a double power series around $\left(x_{0}, y_{0}\right)$. Its convergence can be discussed by letting $\tilde{x}=x-x_{0}$ and $\tilde{y}=y-y_{0}$, and considering the double power series $\sum \sum_{(k, \ell)} c_{k, \ell} \tilde{x}^{k} \tilde{y}^{\ell}$.

Typical sets of points $(x, y)$ in $\mathbb{R}^{2}$ for which a double power series is convergent are illustrated by the following examples: 
Example A.8.1. [28].

(i) Let $c_{k, \ell}:=(k+1)^{(k+1)}(\ell+1)^{(\ell+1)}$ for $(k, \ell) \succeq(0,0)$, and let $(x, y) \in \mathbb{R}^{2}$. If $\left|c_{k, \ell} x^{k} y^{\ell}\right|>$ 1 for all $(k, \ell) \in \mathbb{N}_{0}^{2}$ satisfying $k>1 /|x|^{k+1}-1$ and $\ell>1 /|y|^{\ell+1}-1$, and so by the $(k, \ell)$ th Term Test (Theorem A.3.1), the double power series is divergent. Similarly, if $x \neq 0$ and $y=0$, then the series $\sum_{k=0}^{\infty} c_{k, 0} x^{k}$ is divergent, and if $x=0$ and $y \neq 0$, then the series $\sum_{\ell=0}^{\infty} c_{0, \ell} y^{\ell}$ is divergent. Thus we see that the double power $\sum \sum_{(k, \ell)} c_{k, \ell} x^{k} y^{\ell}$ is convergent if and only if $(x, y)=(0,0)$.

(ii) Let $c_{k, \ell}:=1 / k ! \ell !$ for $(k, \ell) \succeq(0,0)$. It follows from Example A.3.1 (ii) that the double power series $\sum \sum_{(k, \ell)} c_{k, \ell} x^{k} y^{\ell}$ is convergent for all $(x, y) \in \mathbb{R}^{2}$.

(iii) Let a and $b$ be nonzero real numbers, and let $c_{k, \ell}:=a^{k} b^{\ell}$ for $(k, \ell) \succeq(0,0)$ It follows from Example A.3.1 (i) that the double power series $\sum \sum_{(k, \ell)} c_{k, \ell} x^{k} y^{\ell}$ is convergent if and only if $|a x|<1$ and $|b y|<1$, that is, $|x|<1 /|a|$ and $|y|<1 /|b|$.

(iv) $\operatorname{For}(k, \ell) \succeq(0,0)$, let

$$
c_{k, \ell}:=\left\{\begin{array}{lll}
1 & \text { if } & k=1 \\
0 & \text { if } & k \neq 1
\end{array}\right.
$$

Then for $(x, y) \in \mathbb{R}^{2}$, the partial double sums of the double power series $\sum \sum_{(k, \ell)} c_{k, \ell} x^{k} y^{\ell}$ are $A_{0, n}(x, y):=0$ for $n \geq 0$, and

$$
A_{m, n}(x, y)=x \sum_{\ell=0}^{n} y^{\ell} \quad \text { for } \quad(m, n) \succeq(1,0) .
$$

Consequently, the double power series converges absolutely if $x=0$ or $|y|<1$, while it diverges if $x \neq 0$ and $|y| \geq 1$. It follows that the set of $(x, y) \in \mathbb{R}^{2}$ for which this double power series converges is the horizontal strip $\mathbb{R} \times(-1,1)$ together with the y-axis, as shown in Figure A.2. On this set, the convergence is absolute.

(v) $\operatorname{For}(k, \ell) \succeq(0,0)$, let

$$
c_{k, \ell}:=\left\{\begin{array}{lll}
1 & \text { if } \quad k=\ell \\
0 & \text { if } \quad k \neq \ell
\end{array}\right.
$$

Then for $(x, y) \in \mathbb{R}^{2}$, the partial double sums of the double power series $\sum \sum_{(k, \ell)} c_{k, \ell} x^{k} y^{\ell}$ are

$$
A_{m, n}(x, y)=\sum_{p=0}^{\min \{m, n\}}(x y)^{p} \quad \text { for } \quad(m, n) \succeq(0,0) .
$$



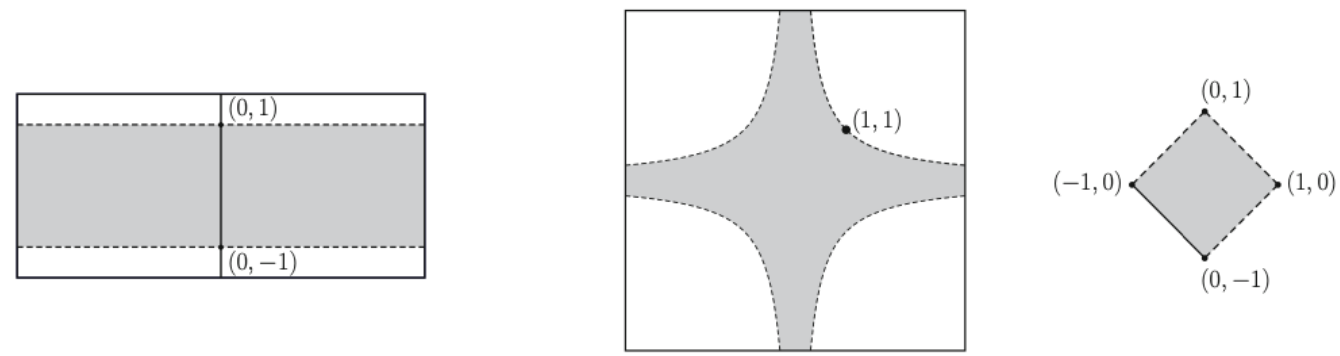

Figure A.2: Illustration of sets of convergence: The horizontal strip and the y-axis, the region bounded by rectangular hyperbolas, and the diamond-shaped region on which the double power series in Examples A.8.1 (iv), (v), and converge, respectively.

Using the fact that the geometric series $\sum_{p} a^{p}$ converges absolutely if $|a|<1$, while it diverges if $|a| \geq 1$, we see that the double power series converges absolutely if $|x y|<1$, while it diverges if $|x y| \geq 1$. Thus the subset of $\mathbb{R}^{2}$ on which this double power series converges is precisely the region $\left\{(x, y) \in \mathbb{R}^{2}:-1<x y<1\right\}$ bounded by the rectangular hyperbolas $x y=1$ and $x y=-1$, as shown in Figure A.2. On this set, the convergence is absolute.

(vi) $c_{k . \ell}:=(k+\ell) / k ! \ell !$ for $(k, \ell) \succeq(0,0)$, and let $(x, y) \in \mathbb{R}^{2}$. As in the proof of part (iii) of Theorem A.5.3,

$$
\sum_{k=0}^{m} \sum_{\ell=0}^{n}\left|c_{k, \ell}\right||x|^{k}|y|^{\ell} \leq \sum_{j=0}^{m+n} \sum_{k=0}^{j} \frac{j !|x|^{k}|y|^{j-k}}{k !(j-k) !}=\sum_{j=0}^{m+n}(|x|+|y|)^{j}
$$

for $(m, n) \succeq(0,0)$, whereas

$$
\sum_{k=0}^{n} \sum_{\ell=0}^{n}\left|c_{k, \ell}\right||x|^{k}|y|^{\ell} \geq \sum_{j=0}^{n} \sum_{k=0}^{j} \frac{j !|x|^{k}|y|^{j-k}}{k !(j-k) !}=\sum_{j=0}^{n}(|x|+|y|)^{j}
$$

for $n \geq 0$. Thus, in view of Example A.3.1 ( $i)$, we see that the double power series $\sum \sum_{(k, \ell)} c_{k, \ell} x^{k} y^{\ell}$ converges absolutely if and only if $|x|+|y|<1$. The subset of $\mathbb{R}^{2}$ on which this double power series converges absolutely is the diamond-shaped region $\left\{(x, y) \in \mathbb{R}^{2}:|x|+|y|<1\right\}$. It turns out that the set on which the double series converges is this diamond-shaped region together with the open line segment joining $(-1,0)$ and $(0,-1)$, as shown in Figure A.2.

The above examples show that the set of all $(x, y) \in \mathbb{R}^{2}$ for which a double power series $\sum \sum_{(k, \ell)} c_{k, \ell} x^{k} y^{\ell}$ converges absolutely can be of a varied nature. This is in contrast 
to the convergence of a (single) power series for which the corresponding subset of $\mathbb{R}$ is always an interval. In this connection, we recall following result for (single) power series.

Fact A.8.1. (Abel's Lemma) [28]. Let $x_{0} \in \mathbb{R}$ and let $c_{k} \in \mathbb{R}$ for $k \geq 0$. If the set $\left\{c_{k} x_{0}^{k}: k \geq\right.$ $0\}$ is bounded, then the power series $\sum_{k=0}^{\infty} c_{k} x^{k}$ is absolutely convergent for every $x \in \mathbb{R}$ with $|x|<\left|x_{0}\right|$.

This leads to the following fundamental result about the (absolute) convergence of a (single) power series.

Fact A.8.2. [28]. Either a power series $\sum_{k} c_{k} x^{k}$ converges absolutely for all $x \in \mathbb{R}$, or there is a non-negative real number $r$ such that it converges absolutely for all $x \in \mathbb{R}$ with $|x|<r$ and diverges for all $x \in \mathbb{R}$ with $|x|>r$.

The radius of convergence of the power series is defined to be $\infty$ in the former case, and it is defined to be the unique non-negative real number $r$ with the stated properties in the latter case. We will now attempt to obtain analogues of the above results for double power series.

Lemma A.8.1. (Abel's Lemma for Double Power Series)[28]. Let $\left(x_{0}, y_{0}\right)$ be in $\mathbb{R}^{2}$ and let $c_{k, \ell} \in \mathbb{R}$ for $(k, \ell) \succeq(0,0)$. If the set $\left\{c_{k, \ell}: x_{0}^{k} y_{0}^{\ell}:(k, \ell) \succeq(0,0)\right\}$ is bounded, then the double power series $\sum \sum_{(k, \ell)} c_{k, \ell} x^{k} y^{\ell}$ is absolutely convergent for every $(x, y) \in \mathbb{R}^{2}$ with $|x|<\left|x_{0}\right|$ and $|y|<\left|y_{0}\right|$.

Proof. If $x_{0}=0$ or $y_{0}=0$, then there is nothing to prove. Suppose $x_{0} \neq 0$ and $y_{0} \neq 0$. Let $\alpha \in \mathbb{R}$ be such that $\left|c_{k, \ell} x_{0}^{k} y_{0}^{\ell}\right| \leq \alpha$ for all $(k, \ell) \succeq(0,0)$. Given any $(x, y) \in \mathbb{R}^{2}$ with $|x|<\left|x_{0}\right|$ and $|y|<\left|y_{0}\right|$, let $\beta:=|x| /\left|x_{0}\right|$ and $\gamma:=|y| /\left|y_{0}\right|$. Then

$$
\left|c_{k, \ell} x^{k} y^{\ell}\right|=\left|c_{k, \ell} x_{0}^{k} y_{0}^{\ell}\right| \beta^{k} \gamma^{\ell} \leq \alpha \beta^{k} \gamma^{\ell} \quad \text { for all } \quad(k, l) \succeq(0,0) .
$$

Since $\beta<1$ and $\gamma<1$, the geometric double series $\sum \sum_{(k, \ell)} c_{k, \ell} \beta^{k} \gamma^{\ell}$ is convergent (See Example A.3.1 (i).) By the Comparison Test for the Double Series it follows the $\sum \sum_{(k, \ell)} c_{k, \ell} x^{k} y^{\ell}$ is absolutely convergent.

Theorem A.8.1. [28]. Either the double series $\sum \sum_{(k, \ell)} c_{k, \ell} x^{k} y^{\ell}$ converges absolutely for all $(x, y) \in \mathbb{R}^{2}$, or there are non-negative real numbers $r$ and s such that it converges absolutely for all $(x, y) \in \mathbb{R}^{2}$ with $|x|<r$ and $|y|<s$, while the $\operatorname{set}\left\{c_{k, \ell} x^{k} y^{\ell}:(k, \ell) \succeq(0,0)\right\}$ is unbounded for all $(x, y) \in \mathbb{R}^{2}$ with $|x|>r$ and $|y|>s$.

Proof. For $(x, y) \in \mathbb{R}^{2}$, let $C_{x, y}=\left\{c_{k, \ell} x^{k} y^{\ell}:(k, \ell) \succeq(0,0)\right\}$. Consider $E:=\{(x, y) \in$ $\mathbb{R}^{2}: C_{x, y}$ is bounded $\}$. For $(x, y) \in \mathbb{R}^{2}$, note that $(x, y) \in E$ if and only if $(|x|,|y|) \in E$. If $E=\mathbb{R}^{2}$, then given any $(x, y) \in \mathbb{R}^{2}$, we can find $\left(x_{0}, y_{0}\right) \in E$ such that $|x|<\left|x_{0}\right|$ and $|y|<$ $\left|y_{0}\right|$. Since the set $C_{x_{0}, y_{0}}$ is bounded, by Lemma A.8.1, the double series $\sum \sum_{(k, \ell)} c_{k, \ell} x^{k} y^{\ell}$ is 
absolutely convergent. Next, suppose $E \neq \mathbb{R}$. The set $E$ is nonempty since $(0,0)$. Then $E$ has a boundary point $\left(x^{*}, y^{*}\right) \in \mathbb{R}^{2}$. Define $r:=\left|x^{*}\right|$ and $s:=\left|y^{*}\right|$. Let $(x, y) \in \mathbb{R}^{2}$ with $|x|<r$ and $|y|<s$. By the definition of a boundary point, there is a sequence in $\mathrm{E}$ converging to $\left(x^{*}, y^{*}\right)$, and so we can find $\left(x_{0}, y_{0}\right) \in E$ such that $|x|<\left|x_{0}\right|$ and $|y|<\left|y_{0}\right|$. Hence by Lemma A.8.1, $\sum \sum_{(k, \ell)} c_{k, \ell} x^{k} y^{\ell}$ is absolutely convergent. On the other hand, let $(x, y) \in \mathbb{R}^{2}$ with $|x|>r$ and $|y|>s$. By the definition of a boundary point, there is a sequence in $\mathbb{R}^{2} \backslash E$ converging to $\left(x^{*}, y^{*}\right)$, and so we may find $\left(x_{1}, y_{1}\right) \in \mathbb{R}^{2}$ such that $\left|x_{1}\right|<|x|$ and $\left|y_{1}\right|<|y|$. Now since the set $C_{x_{1}, y_{1}}$ is unbounded, it follows that the set $C_{x, y}$ is also unbounded. This proves the existence of non-negative real numbers $r$ and $s$ with the desired properties

If a double power series $\sum \sum_{(k, \ell)} c_{k, \ell} x^{k} y^{\ell}$ is absolutely convergent for all $(x, y) \in \mathbb{R}^{2}$, then we say that its biradius of convergence is $(\infty, \infty)$; otherwise, a pair $(r, s)$ of nonnegative real numbers is said to be a biradius of convergence of the double power series, provided the double series converges absolutely for all $(x, y) \in \mathbb{R}^{2}$ with $|x|<r$ and $|y|<s$, while the set $C_{x, y}:=\left\{c_{k, \ell} x^{k} y^{\ell}:(k, \ell) \succeq(0,0)\right\}$ is unbounded for all $(x, y) \in \mathbb{R}^{2}$ with $|x|>r$ and $|y|>s$. This phenomenon is illustrated in Figure A.3. Proposition A.8.1 says that every double power series has a biradius of convergence.

Remark A.8.1. [28].

(i) It is interesting to observe that if $r$ is the radius of convergence of a (single) power series, then the power series diverges for all $x \in \mathbb{R}$ with $|x|>r$, whereas if $(r, s)$ is a biradius of convergence of a double power series, then the set $C_{x, y}:=\left\{c_{k, \ell} x^{k} y^{\ell}\right.$ : $(k, \ell) \succeq(0,0)\}$ is unbounded for all $(x, y) \in \mathbb{R}^{2}$ with $|x|>r$ and $|y|>s$. The unboundedness of the set $C_{x, y}$ cannot be replaced by the divergence of the double power series at $(x, y)$, as the following example shows. Let $c_{0,0}:=1, c_{k, 0}=c_{0, \ell}:=1$ for all $k, \ell \in \mathbb{N}, c_{1,1}:=-1, c_{k, 1}=c_{1, \ell}:=-1 / 2$ for all $k, \ell \geq 2$, and $c_{k, \ell}:=0$ for all $(k, \ell) \succeq(2,2)$. If $A_{m, n}(x, y)$ denotes the $(m, n)$ th partial double sum of $\sum \sum_{(k, \ell)} c_{k, \ell} x^{k} y^{\ell}$, then $A_{0,0}(x, y)=1$ and for $(m, n) \in \mathbb{N}^{2}$, we have

$$
A_{m, 0}(x, y)=\sum_{k=0}^{m} x^{k}, \quad A_{0, n}(x, y)=\sum_{\ell=0}^{n} y^{\ell}
$$

and

$$
A_{m, n}(x, y)=1+\left(1-\frac{y}{2}\right) \sum_{k=0}^{m} x^{k}+\left(1-\frac{x}{2}\right) \sum_{\ell=0}^{n} y^{\ell}
$$

It is easy to see that the double power series converges absolutely for all $(x, y) \in \mathbb{R}^{2}$ with $|x|<1$ and $|y|<1$, and it diverges to $\infty$ for all $(x, y)$ with $x \geq 1$ and $y \geq 1$ except for $(x, y)=(2,2)$. At $(2,2)$, a peculiar phenomenon occurs: Since $c_{k, 0} 2^{k} 2^{0}=2 k$ 


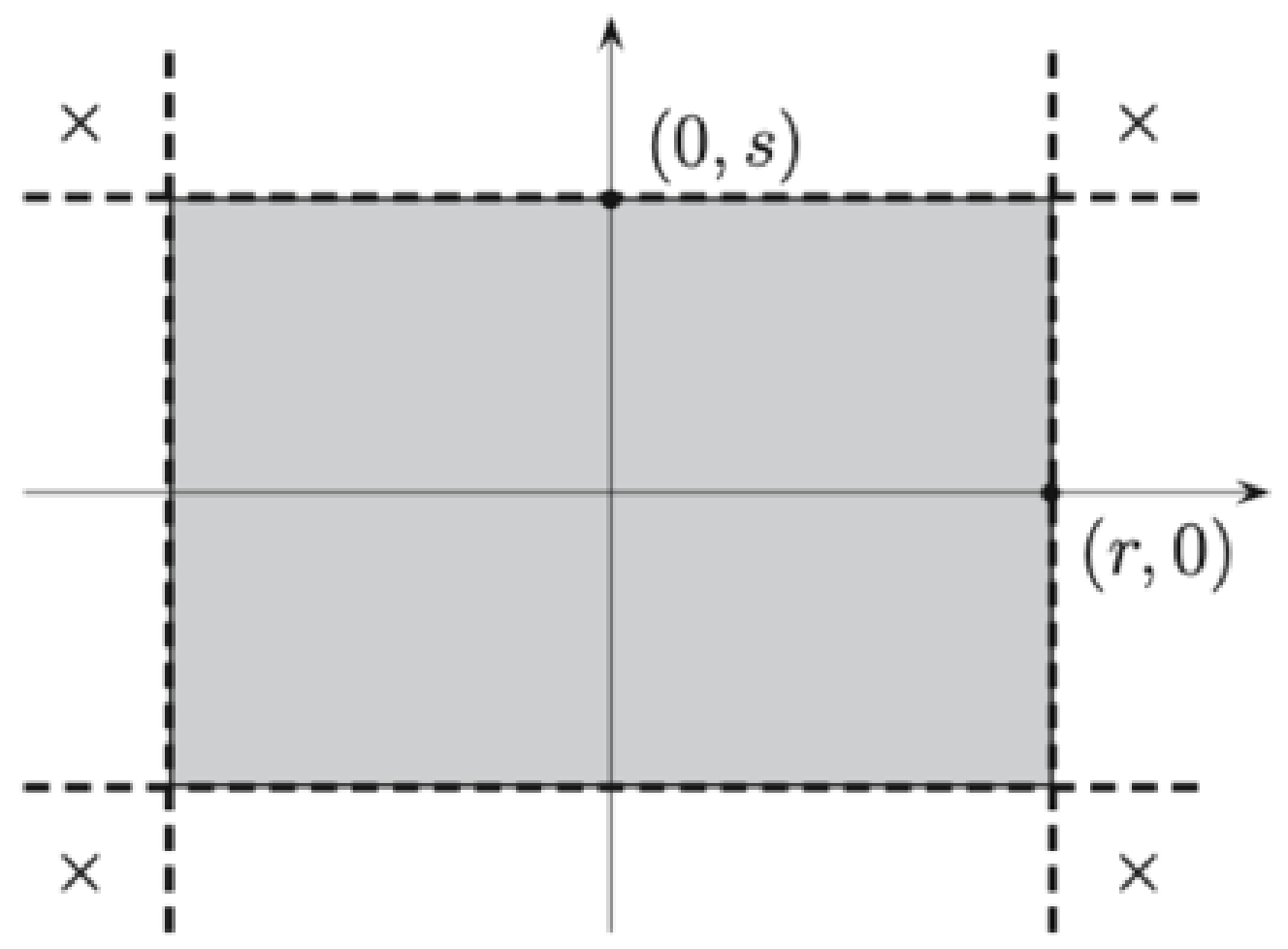

Figure A.3: When $(r, s)$ is a biradius of convergence of a double power series, it converges absolutely in the shaded rectangle, while the set of its terms is unbounded in the four quadrangles marked by $\times$.

and $c_{0, \ell} 2^{0} 2^{\ell}=2 \ell$ for all $(k, \ell) \in \mathbb{N}^{2}$, we see that the set $C_{2,2}$ is unbounded, but since $A_{m, n}(2,2)=1$ for all $(m, n) \in \mathbb{N}^{2}$, we see that the double power series converges to 1 at (2,2). It follows that there are no non-negative numbers $r$ and s such that the double power series converges absolutely for all $(x, y) \in \mathbb{R}^{2}$ with $|x|<r$ and $|y|<s$, and it diverges for all $(x, y) \in \mathbb{R}^{2}$ with $|x|>r$ and $|y|>s$.

(ii) The radius of convergence of a (single) power series is unique. However, a double power series may have several biradii of convergence. For example, let $c_{k, \ell}:=1$ if $k=\ell$ and $c_{k, \ell}:=0$ if $k \neq \ell$ for $(k, \ell) \succeq(0,0)$. Then the double power series $\sum \sum_{(k, \ell)} c_{k, \ell} x^{k} y^{\ell}=\sum_{k=0}^{\infty} x^{k} y^{k}$ converges absolutely if $|x y|<1$. On the other hand, if $|x y|>1$, then the set $C_{x, y}:=\left\{x^{k} y^{k}: k \geq 0\right\}$ is unbounded. It follows that $(t, 1 / t)$ is a biradius of convergence for each positive real number $t$.

It is therefore important to find all biradii of convergence, or failing this, as many biradii of convergence as possible, in order to obtain a fuller picture of the convergence 
behavior of a double power series.

If $r$ is the radius of convergence of a (single) power series, then the set $(-r, r)$ is known as the interval of convergence of the power series. It is the largest open subset of $\mathbb{R}$ in which the power series is absolutely convergent. Analogously, the domain of convergence of a double power series is defined to be the set of all $(x, y) \in \mathbb{R}^{2}$ such that the double power series converges absolutely at every point in some open square centered at $(x, y)$. Note that if $\mathbb{D}$ is the domain of convergence of a double power series, then $D$ is an open subset of $\mathbb{R}^{2}$ and moreover, $(x, y) \in \mathbb{D}$ if and only if $(|x|,|y|) \in \mathbb{D}$. It follows from the Comparison Test and Lemma A.8.1 that $\left(x_{0}, y_{0}\right) \in \mathbb{R}^{2}$ belongs to the domain of convergence of $\sum \sum_{(k, \ell)} c_{k, \ell} x^{k} y^{\ell}$ if and only if the set $C_{x, y}:=\left\{c_{k, \ell} x^{k} y^{\ell}:(k, \ell) \succeq(0,0)\right\}$ is bounded for every $(x, y)$ in some open square centered at $\left(x_{0}, y_{0}\right)$. It also follows that the domain of convergence of a double power series is empty if and only if $(0,0)$ is a biradius of convergence of that double power series.

In the following table we give the domains of convergence and biradii of convergence of the double power series considered in Example A.8.1.

\begin{tabular}{|c|c|c|}
\hline Double Power Series & Domain of Convergence & Biradii of Convergence \\
\hline \hline$\sum_{(k, \ell)} \sum^{k} \ell^{\ell} x^{k} y^{\ell}$ & $\phi$ & $(0,0)$ \\
\hline$\sum_{(k, \ell)} \frac{1}{k ! \ell !} x^{k} y^{\ell}$ & $\mathbb{R}^{2}$ & $(\infty, \infty)$ \\
\hline$\sum_{(k, \ell)} \sum^{k} b^{\ell} x^{k} y^{\ell}$ & $\left\{(x, y) \in \mathbb{R}^{2}:|x|<\frac{1}{a} \quad\right.$ and $\left.\quad|y|<\frac{1}{b}\right\}$ & $\begin{array}{l}\left(r, \frac{1}{|b|}\right) \text { for } 0 \leq r \leq \frac{1}{|a|} \\
\left(\frac{1}{|a|}, s\right) \text { for } 0 \leq s \leq \frac{1}{|b|}\end{array}$ \\
\hline$x \sum_{\ell=0}^{\infty} y^{\ell}$ & $\left\{(x, y) \in \mathbb{R}^{2}:|y|<1\right\}$ & $(r, 1)$ for $0 \leq r<\infty$ \\
\hline$\sum_{k=0}^{\infty}(x y)^{k}$ & $\left\{(x, y) \in \mathbb{R}^{2}:|x y|<1\right\}$ & $\left(t, \frac{1}{t}\right)$ for $0 \leq t<\infty$ \\
\hline$\sum_{(k, \ell)} \frac{(k+\ell) !}{k ! \ell !} x^{k} y^{\ell}$ & $\left\{(x, y) \in \mathbb{R}^{2}:|x|+|y|<1\right\}$ & $(t, t-1)$ for $0 \leq t<1$ \\
\hline
\end{tabular}

The above examples are typical and exhibit the variety of shapes that a domain of convergence of a double power series can have. The example in the penultimate row of the above table shows that such a domain $\mathbb{D}$ need not be a convex subset of $\mathbb{R}^{2}$. However, according to a result of Fabry (1902), the domain of convergence of every double power series is log-convex, that is, it is an open subset $\mathbb{D}$ of $\mathbb{R}^{2}$ such that $\{(\ln |x|, \ln |y|):(x, y) \in$ $\mathbb{D}$ and $x y \neq 0\}$ is a convex subset of $\mathbb{R}^{2}$. 


\section{A.9 Taylor Double Series and Taylor Series}

Let $\mathbb{D} \subseteq \mathbb{R}^{2},\left(x_{0}, y_{0}\right)$ be an interior point of $\mathbb{D}$ and let $f: \mathbb{D} \rightarrow \mathbb{R}$ be such that all partial derivatives of $f$ of all orders exist and are continuous on a square neighborhood of $\left(x_{0}, y_{0}\right)$. In analogy with the Taylor series of a function of one variable, the double power series

$$
\sum_{(k, \ell)} \frac{\partial^{k+\ell} f}{\partial x^{k} \partial y^{\ell}}\left(x_{0}, y_{0}\right) \frac{\left(x-x_{0}\right)^{k}}{k !} \frac{\left(y-y_{0}\right)^{\ell}}{\ell !} .
$$

is called the Taylor double series of $f$ around $\left(x_{0}, y_{0}\right)$. Note that the coefficients of this double power series are

$$
c_{k, \ell}:=\frac{1}{k ! \ell !} \frac{\partial^{k+\ell} f}{\partial x^{k} \partial y^{\ell}}\left(x_{0}, y_{0}\right) \text { for } \quad(k, \ell) \succeq(0,0) .
$$

We observe that for $n=0,1,2, \ldots$, the $n t h$ partial sum of the diagonal series $\sum_{j=0} c_{j}(x, y)$ corresponding to the above double series is

$$
\begin{aligned}
\sum_{j=0}^{n} c_{j}(x, y) & =\sum_{j=0}^{n} \sum_{\substack{k \geq 0 \\
k+\ell=j}} \sum_{\substack{\ell \geq 0 \\
y^{k+\ell} f}}\left(x_{0}, y_{0}\right) \frac{\left(x-x_{0}\right)^{k}}{k !} \frac{\left(y-y_{0}\right)^{\ell}}{\ell !} \\
& =\sum_{j=0}^{n} \sum_{k=0}^{j} \frac{\partial^{j} f}{\partial x^{k} \partial y^{j-k}}\left(x_{0}, y_{0}\right) \frac{\left(x-x_{0}\right)^{k}}{k !} \frac{\left(y-y_{0}\right)^{j-k}}{(j-k) !}
\end{aligned}
$$

which is in fact $n$th bivariate Taylor polynomial $P_{n}(x, y)$ of $f$ around $\left(x_{0}, y_{0}\right)$.

$$
\sum_{j=0}^{\infty} c_{j}(x, y) \quad \text { where } \quad c_{j}(x, y):=\sum_{\substack{k \geq 0 \\ k+\ell=j}} \sum_{\substack{\ell \geq 0 \\ k}} c_{k, \ell}\left(x-x_{0}\right)^{k}\left(y-y_{0}\right)^{\ell} \quad \text { for } j \geq 0
$$

is called the Taylor series of $f$ around $\left(x_{0}, y_{0}\right)$. Thus the Taylor series of a function of two variables is the diagonal series corresponding to its Taylor double series.

An important question one would like to consider is whether the Taylor double series and/or the Taylor series of $f$ around $\left(x_{0}, y_{0}\right)$ converges (absolutely) at a given point $(x, y) \in$ $\mathbb{R}^{2}$, and if so, then whether the corresponding double sum and/or the corresponding sum is equal to $f(x, y)$, provided $(x, y) \in \mathbb{D}$. If $(x, y):=\left(x_{0}, y_{0}\right)$, then each partial double sum of the Taylor double series of $f$ around $\left(x_{0}, y_{0}\right)$ as well as each partial sum of the Taylor series of $f$ around $\left(x_{0}, y_{0}\right)$ is obviously equal to $f\left(x_{0}, y_{0}\right)$, and so our question has an affirmative answer if $(x, y)=\left(x_{0}, y_{0}\right)$. It is, however, possible that for each $(x, y) \in \mathbb{D} \backslash\left\{\left(x_{0}, y_{0}\right)\right\}$, both the Taylor double series and the Taylor series of $f$ around $\left(x_{0}, y_{0}\right)$ converge but not to $f(x, y)$. For instance, let $f: \mathbb{R}^{2} \rightarrow \mathbb{R}$ be defined by

$$
f(x, y):=\left\{\begin{array}{lll}
e^{-\frac{1}{\left(x^{2}+y^{2}\right)}} & \text { if } \quad(x, y) \neq(0,0) \\
0 & \text { if } \quad(x, y)=(0,0)
\end{array}\right.
$$


By considering the function $g: \mathbb{R} \rightarrow \mathbb{R}$ given by $g(0):=0$ and $g(t):=e^{-1} / t^{2}$, and noting that $g^{(k)}(0)=0$ for all $k \in \mathbb{N}$, it can be seen that

$$
\frac{\partial^{k+\ell} f}{\partial x^{k} \partial y^{\ell}}(0,0)=0 \quad \text { for all } \quad(k, \ell) \succeq(0,0) .
$$

Thus the Taylor double series of $f$ around $(0,0)$ as well as the Taylor series of $f$ around $(0,0)$ is identically zero, and neither converges to $f(x, y)$ at any $(x, y) \neq(0,0)$.

If the Taylor double series of $f$ around $\left(x_{0}, y_{0}\right)$ converges absolutely at $(x, y) \in \mathbb{R}^{2}$, then by part (iii) of Theorem A.6.3, the Taylor series of $f$ around $\left(x_{0}, y_{0}\right)$ also converges absolutely at $(x, y)$. But the converse is not true, as we will see in Example A.9.1 (ii). For $(x, y) \in \mathbb{D}$ and $n=0,1,2, \ldots$, let $R_{n}(x, y):=f(x, y)-P_{n}(x, y)$ and note that the Taylor series of $f$ around $\left(x_{0}, y_{0}\right)$ converges to $f(x, y)$ if and only if $R_{n}(x, y) \rightarrow 0$ as $n \rightarrow \infty$. The following results give sufficient conditions for the absolute convergence on $\mathbb{R}^{2}$ of the Taylor double series of a function and for deciding whether it converges to the function itself.

Theorem A.9.1. [28]. Let $\mathbb{D}$ be an open subset of $\mathbb{R}^{2}$, and let $\left(x_{0}, y_{0}\right) \in \mathbb{D}$. Suppose $f: \mathbb{D} \rightarrow \mathbb{R}$ has continuous partial derivatives of all orders on $\mathbb{D}$, and there are positive real numbers $M_{0}, \alpha_{0}$, and $\beta_{0}$ such that

$$
\left|\frac{\partial^{k+\ell} f}{\partial x^{k} \partial y^{\ell}}\left(x_{0}, y_{0}\right)\right| \leq M_{0} \alpha_{0}^{k} \beta_{0}^{\ell} \quad \text { for all } \quad(k, \ell) \succeq(0,0) .
$$

Then the Taylor double series of $f$ and the Taylor series of $f$ around $\left(x_{0}, y_{0}\right)$ converge absolutely for all $(x, y) \in \mathbb{R}^{2}$. Moreover, both of these converge to $f(x, y)$, provided the line $L$ joining $\left(x_{0}, y_{0}\right)$ and $(x, y)$ lies in $\mathbb{D}$ and there are positive real numbers $M, \alpha$, and $\beta$ such that

$$
\left|\frac{\partial^{k+\ell} f}{\partial x^{k} \partial y^{\ell}}(\tilde{x}, \tilde{y})\right| \leq M \alpha^{k} \beta^{\ell} \quad \text { for all } \quad(\tilde{x}, \tilde{y}) \in L \quad \text { and all } \quad(k, \ell) \succeq(0,0) .
$$

Proof. Since the exponential double series

$$
\sum_{(k, \ell)} \frac{\left[\alpha\left(x-x_{0}\right)\right]^{k}}{k !} \frac{\left[\beta\left(y-y_{0}\right)\right]^{\ell}}{\ell !}
$$

converges absolutely for all $(x, y) \in \mathbb{R}^{2}$, the Comparison Test for Double Series shows that the Taylor double series of $f$ around $\left(x_{0}, y_{0}\right)$ converges absolutely for all $(x, y) \in \mathbb{R}^{2}$. Consequently, by part (iii) of Theorem A.6.3, the corresponding diagonal series, namely the Taylor series of $f$ around $\left(x_{0}, y_{0}\right)$, also converges absolutely for all $(x, y) \in \mathbb{R}^{2}$. 
Next, let $(x, y) \in \mathbb{D}$ be such that the line $L$ joining $\left(x_{0}, y_{0}\right)$ and $(x, y)$ lies in $\mathbb{D}$ and there are positive real numbers $M, \alpha$, and $\beta$ such that

$$
\left|\frac{\partial^{k+\ell} f}{\partial x^{k} \partial y^{\ell}}(\tilde{x}, \tilde{y})\right| \leq M \alpha^{k} \beta^{\ell} \quad \text { for all } \quad(\tilde{x}, \tilde{y}) \in L \quad \text { and all } \quad(k, \ell) \succeq(0,0) .
$$

Then by the Classical Version of the Bivariate Taylor Theorem, there is $(c, d) \in L$ such that

$$
R_{n}(x, y):=f(x, y)-P_{n}(x, y)=\sum_{\substack{k \geq 0 \\ k+\ell=n+1}} \frac{\partial^{n+1} f}{\partial x^{k} \partial y^{\ell}}(c, d) \frac{\left(x-x_{0}\right)^{k}}{k !} \frac{\left(y-y_{0}\right)^{\ell}}{\ell !}
$$

and consequently,

$$
\begin{aligned}
R_{n}(x, y):=f(x, y)-P_{n}(x, y) & =\sum_{\substack{k \geq 0 \\
k+\ell=n+1}} M \frac{\left(\alpha\left|x-x_{0}\right|\right)^{k}}{k !} \frac{\left(\beta\left|y-y_{0}\right|\right)^{\ell}}{\ell !} \\
& =M \sum_{k=0}^{n+1} \frac{\left(\alpha\left|x-x_{0}\right|\right)^{k}}{k !} \frac{\left(\beta\left|y-y_{0}\right|\right)^{n+k-1}}{(n+k-1) !} \\
& =\frac{M\left(\alpha\left|x-x_{0}\right|+\beta\left|y-y_{0}\right|\right)^{n+1}}{(n+1) !} .
\end{aligned}
$$

This implies that $R_{n}(x, y) \rightarrow 0$ as $n \rightarrow \infty$. Hence the Taylor series of $f$ about $\left(x_{0}, y_{0}\right)$ converges to $f(x, y)$ at $(x, y)$. Finally, the absolute convergence of the Taylor double series of $f$ around $\left(x_{0}, y_{0}\right)$ at $(x, y)$ implies that its double sum is also equal to $f(x, y)$.

Example A.9.1. [28].

(i) Let $\mathbb{D}:=\left\{(x, y) \in \mathbb{R}^{2}: x<1\right.$ and $\left.y<1\right\}$ and let $f: \mathbb{D} \rightarrow \mathbb{R}$ be defined by $f(x, y):=1 /(1-x)(1-y)$. It is easy to see that

$$
\frac{\partial^{k+\ell} f}{\partial x^{k} \partial y^{\ell}}(0,0)=k ! \ell ! \quad \text { for all } \quad(k, \ell) \succeq(0,0) .
$$

Hence the Taylor double series of $f$ around $(0,0)$ is the geometric double series $\sum \sum_{(k, \ell)} x^{k} y^{\ell}$. As we have seen in Example A.3.1 (i), it converges absolutely if $|x|<1$ and $|y|<1$, while it diverges otherwise; moreover, if $|x|<1$ and $|y|<1$, then the double sum is $1 /(1-x)(1-y)=f(x, y)$. The Taylor series of $f$ around $(0,0)$ is

$$
\sum_{j=0}^{\infty} c_{j}(x, y) \quad \text { where } \quad c_{j}(x, y):=\sum_{k=0}^{j} x^{k} y^{j-k} \quad \text { for } \quad(x, y) \in \mathbb{R}^{2} .
$$


By part (iii) of Theorem A.6.3, it converges absolutely if $|x|<1$ and $|y|<1$, and then its sum is equal to $f(x, y)$. We show that it diverges if $|x| \geq 1$ or $|y| \geq 1$. Assume that $|x| \geq 1$, and let $u:=y / x$. Then

$$
c_{j}(x, y)=x^{j} \sum_{k=0}^{j} u^{j-k}=x^{j}\left(1+u+\cdots+u^{j}\right) \quad \text { for } \quad j \geq 0 .
$$

If $u=1$, then $\left|c_{j}(x, y)\right|=|x|^{j}(j+1) \geq j+1$, and if $u \neq 1$, then

$$
\left|c_{j}(x, y)\right|=\frac{|x|^{j}\left|u^{j+1}-1\right|}{u-1} \geq \frac{\left|u^{j+1}-1\right|}{u-1} \quad \text { for } \quad j \geq 0 .
$$

It follows that $c_{j}(x, y) \nrightarrow 0$ as $j \rightarrow \infty$. Hence the Taylor series of $f$ around $(0,0)$ diverges if $|x| \geq 1$. Similarly, we see that it diverges if $|y| \geq 1$.

(ii) Let $\mathbb{D}:=\left\{(x, y) \in \mathbb{R}^{2}: x+y<1\right\}$ and let $f: \mathbb{D} \rightarrow \mathbb{R}$ be defined by $f(x, y):=$ $1 /(1-x-y)$. It is easy to see that

$$
\frac{\partial^{k+\ell} f}{\partial x^{k} \partial y^{\ell}}(0,0)=(k+\ell) ! \quad \text { for } \quad k, \ell=0,1,2 \ldots .
$$

Hence the Taylor double series off around $(0,0)$ is

$$
\sum_{(k, \ell)} \sum_{k ! \ell !} \frac{(k+\ell) !}{k} y^{\ell}
$$

As shown in Example A.8.1 (vi), this double series converges absolutely if and only if $|x|+|y|<1$. The Taylor series of $f$ around $(0,0)$ is

$$
\sum_{j=0}^{\infty}\left(\sum_{k=0}^{j} \frac{j !}{k !(j-k) !} x^{k} y^{j-k}\right)=\sum_{j=0}^{\infty}(x+y)^{j}
$$

Clearly, this geometric series converges if and only if $|x+y|<1$, and in this case, the convergence is absolute and the sum of the series at $(x, y)$ is equal to $1 /[1-(x+$ $y)]=f(x, y)$. Thus if $(x, y) \in \mathbb{R}^{2}$ satisfies $|x+y|<1 \leq|x|+|y|$, then the Taylor series of $f$ around $(0,0)$ converges absolutely at $(x, y)$, but the Taylor double series of $f$ around $(0,0)$ does not. Since the Taylor series of $f$ around $(0,0)$ is the diagonal series corresponding to the Taylor double series of $f$ around $(0,0)$, it follows from Theorem A.5.3 that if $(x, y) \in \mathbb{R}^{2}$ and $|x|+|y|<1$, then the double sum of the Taylor double series of $f$ around $(0,0)$ at $(x, y)$ is equal to $f(x, y)$. It can be shown that this Taylor double series converges conditionally at $(x, y) \in \mathbb{R}^{2}$ if and only if $x \in(-1,0)$ and $x+y=1$, and then its double sum is equal to $1 / 2$. 
(iii) Let $\mathbb{D}:=\mathbb{R}^{2}$ and let $f: \mathbb{D} \rightarrow \mathbb{R}$ be defined by $f(x, y):=\sin (x+y)$. Letting $g(u):=$ $\sin u$ for $u \in \mathbb{R}$, it is easy to see that for $k, \ell=0,1,2, \ldots$,

$$
\frac{\partial^{k+\ell} f}{\partial x^{k} \partial y^{\ell}}(0,0)=g^{k+\ell}(0)=\left\{\begin{array}{lll}
0 & \text { if } k+\ell \text { is even } \\
(-1)^{(k+\ell-1) / 2} & \text { if } k+\ell \text { is odd }
\end{array}\right.
$$

Hence the Taylor double series of $f$ around $(0,0)$ is

$$
\sum_{(k, \ell)} c_{k, \ell} x^{k} y^{\ell}, \quad \text { where } \quad c_{k, \ell}=\left\{\begin{array}{lll}
0 & \text { if } k+\ell \text { is even } \\
\frac{(-1)^{(k+\ell-1) / 2}}{k ! \ell !} & \text { if } k+\ell \text { is odd } .
\end{array}\right.
$$

The Taylor series of $f$ around $(0,0)$ is

$$
\sum_{j=0}^{\infty} c_{j}(x, y), \quad \text { where } \quad c_{j}(x, y)=\sum_{k=0}^{j} g^{j}(0) \frac{x^{k}}{k !} \frac{y^{j-k}}{(j-k) !}=\frac{g^{j}(0)}{j !}(x+y)^{j},
$$

that is, by $\sum_{j=0}^{\infty}(-1)^{j}(x+y)^{2 j+1} /(2 j+1)$ ! . It follows from Theorem A.9.1 that both the Taylor double series and the Taylor series of $f$ around $(0,0)$ converge absolutely to $f(x, y)$ at all $(x, y) \in \mathbb{R}^{2}$.

(iv) Let $\mathbb{D}:=\mathbb{R}^{2}$ and let $f: \mathbb{D} \rightarrow \mathbb{R}$ be defined by $f(x, y):=\exp (x+y)$. Proceeding as (ii) above, we see that both the Taylor double series $\sum \sum_{(k, \ell)} x^{k} y^{\ell} / k ! \ell !$ of $f$ around $(0,0)$ and the Taylor series $\sum_{j=0}^{\infty}(x+y)^{j} / j$ ! of $f$ around $(0,0)$ converge absolutely to $f(x, y)$ at all $(x, y) \in \mathbb{R}^{2}$.

Remark A.9.1. [28]. Let $\mathbb{D}$ be an open subset of $\mathbb{R}^{2}$ and let $f: \mathbb{D} \rightarrow \mathbb{R}$ be such that all partial derivatives of $f$ of all orders exist and are continuous on $\mathbb{D}$. If for every $\left(x_{0}, y_{0}\right) \in$ $\mathbb{D}$, there are $r>0$ and $s>0$ such that the Taylor double series of $f$ around $\left(x_{0}, y_{0}\right)$ converges absolutely to $f(x, y)$ for all $(x, y) \in \mathbb{D}$ with $\left|x-x_{0}\right|<r$ and $\left|y-y_{0}\right|<s$, then $f$ is said to be real analytic on $\mathbb{D}$. In this case, by part (iii) of Theorem A.6.3, the Taylor series of $f$ around $\left(x_{0}, y_{0}\right)$ also converges absolutely to $f(x, y)$ for all $(x, y) \in \mathbb{D}$ with $\left|x-x_{0}\right|<r$ and $\left|y-y_{0}\right|<s$. Clearly, polynomial functions in two variables are real analytic on $\mathbb{R}^{2}$. Also, using Theorem A.9.1, it can be seen that the functions defined by $f_{1}(x, y):=$ $\sin (x+y)$ and $f_{2}(x, y):=\exp (x+y)$ for $\left(x_{0}, y_{0}\right) \in \mathbb{D}$ are real analytic on $\mathbb{R}^{2}$. In fact, if $\mathbb{D}$ is the domain of convergence of a double power series and if its double sum is denoted by $f(x, y)$ for $(x, y) \in \mathbb{D}$, then the function $f$ is real analytic on $\mathbb{D}$. On the other hand, $a$ function having continuous partial derivatives of all orders on an open subset of $\mathbb{R}^{2}$ need not be real analytic there. Indeed, as noted earlier, it suffices to consider $f: \mathbb{R}^{2} \rightarrow \mathbb{R}$ defined by $f(0,0):=0$ and $f(x, y):=e^{-1 /\left(x^{2}+y^{2}\right)}$ for $(x, y) \neq(0,0)$. 


\section{Bibliography}

[1] George E. Andrews, The Theory of Partitions, Cambridge Mathematical Library, Cambridge University Press, Cambridge, U.K, 1998.

[2] Tom M . Apostol, Introduction to Analytic Number Theory, Springer Science \& Business Media, May 28, 1998.

[3] George E. Andrews, Rogers-Ramanujan Identities for Two-Color Partitions, Indian Journal of Mathematics, Ramanujan Centenary Volume, 29(1987) No. 2, 117-125.

[4] George E. Andrews, Theory of Compositions IV; Mutlicompositions, The Mathematics Student, Special Centenary Volume, 2007, 25-31.

[5] J.B. Conway, Functions of One Complex Variable, Springer, 1995.

[6] M. Elewoday, H. Gingold, and J. Quaintance, Factorizations of Bivariate Taylor Series Via Power Products, preprint, March 2020.

[7] M. Elewoday, H. Gingold, and J. Quaintance, Factorizations of bivariate Taylor series via inverse power products, The Ramanujan Journal, March 2021.

[8] S. Borofsky, Expansions of functions defined by Dirichlet series into infinite series and products, Tohoku Math. J., 34(1930), 263-274.

[9] S. Borofsky, Expansion of analytic functions into infinite products, Annals of Math., 32(1931) No. 1, 23-36.

[10] M. S. Cheema, Vector partitions and combinatorial identities, Journal: Math. Comp., 18(1964), 414-420.

[11] J.French, Vector Partitions, master thesis, East Tennessee State University, May 2018.

[12] J. M. Feld, The expansion of analytic functions in generalized Lambert series, Annals of Math., 33(1932) No. 1, 139-142. 
[13] J. M.Feld, P. Newman, On the representation of analytic functions of several variables as infinite products, Bull. Amer. Math. Soc., 36(1930) No. 4, 284-288.

[14] J. Gallier and J. Quaintance, Differential Geometry and Lie Groups: A Computational Perspective, Springer, 2020.

[15] J. Gallier and J. Quaintance, Linear Algebra and Optimization with Applications to Machine Learning Volume I: Linear Algebra for Computer Vision, Robotics, and Machine Learning, World Scientific, 2019.

[16] H. Gingold, A note on the reduction of operations via power product approximations, Utilitas Math., 37(1990), 79-90.

[17] H. Gingold, Factorization of Matrix Functions and Their Inverses Via Power Product Expansion, Linear Algebra and its Applications, 430(2008) No. 11-12, 2835-3140.

[18] H. Gingold, A. Knopfmacher, D. S. Lubinsky, The zero distribution of the partial products of power expansions, Analysis, 13(1993) No. 1-2, 133-157.

[19] H. Gingold, A. Knopfmacher, Analytic Properties of Power Product Expansions, Can. J. Math, 47(1995) No. 6, 1219-1239.

[20] H. Gingold and J. Quaintance, Combinatorial analysis of integer power product expansions, Online Journal of Analytic Combinatorics, 11(2016).

[21] H. Gingold and J. Quaintance, Factorizations of analytic functions via generalized inverse power product expansions, International Journal of Pure and Applied Mathematics, 97(2014) No. 2, 115-146

[22] H. Gingold and J. Quaintance, Approximations of Analytic Functions via Generalized Power Product Expansion, Journal of Approximation Theory, 188(2014), 1938 .

[23] H. Gingold, H.W. Gould, Michael E. Mays, Power Product Expansions, Utilitas Mathematica, 34(1988), 143-161.

[24] H.Gingold, A note on the reduction of operations via power product approximations, Utilitas Mathematica, 37(1990), 79-90.

[25] H. W. Gould, Combinatorial Identities, A Standardized Set of Tables Listing 500 Binomial Coefficient Summations, Revised Edition, Published by the author, Morgantown, WV, (1972).

[26] E. Grosswald, Topics from the Theory of Numbers, 2nd Edition, Birkhauser, 1984. 
[27] G. H. Hardy, A note on the continuity or discontinuity of a function defined by an infinite product, Proc. London Math. Soc. (2), 7(1909), 40-48.

[28] S. Ghorpade, B. Limaye, A Course in Multivariable Calculus and Analysis, Springer, 2010.

[29] F. Hertzog, Remarks on a paper by Kolberg, Nordisk Mathematisk Tidskrift, 10(1962), 78-79.

[30] J.E Humphreys, Introduction to Lie Algebras and Representation Theory Springer New York, 2012.

[31] H. Indlekofer, R. Warlimont, Remarks on the infinite product representations of holomorphic functions, Publ. Math. Debrecen, 41(1992), 263-276.

[32] O. Kolberg, A property of the coefficients in a certain product expansion of the exponential function, Nordisk Mathematisk Tidskrift, 10(1960), 33-34.

[33] A. Knopfmacher, Infinite product factorization of analytic functions, J. Math. Anal. Appl., 62(1991) No. 2, 526-536.

[34] A. Knopfmacher, Generalized series expansions of functions, Progress in Approximation Theory, [Nevai, P. and Pinkus, A., eds], Academic Press, USA, 1991, 513533.

[35] A. Knopfmacher and L. Lucht, The radius of convergence of power product expansions, Analysis, 11(1991) No. 1, 91-99.

[36] A. Knopfmacher and J. N. Ridely, Reciprocal Sums Over partitions and Compositions, SIAM J. Disc. Math., 6(1993), 388-399.

[37] A. Knopfmacher, A. M. Odlyzko, B. Pittel, B. Richmond, D. Stark, G. Szekeres, and N.Wormald, The asymptotic number of set partitions with unequal block sizes, The Electronic Journal of Combinatorics, 6(1999), 1-36.

[38] J. Morrow, Double Series, class notes for Honors Advanced Calculus, Winter 2012, https://sites.math.washington.edu/ morrow/335_12/doubleSeries.pdf

[39] M. Mursaleen, S.A. Mohiuddine, Convergence Methods for Double Sequences and Applications, Springer, 2013.

[40] K O’Meara, J Clark, C Vinsonhale, Advanced Topics in Linear Algebra, Oxford University Press, USA, 2011.

[41] J. F. Ritt, Representation of Analytic functions in infinite product expansions, Math.Z., 32(1930), 1-3. 
[42] R. Silverman, Introductory Complex Analysis, Dover Publications, New York, 1972.

[43] J. Thunder, Basic Facts on Infinite Products, class notes for Math 680, Fall 2020, http://www.math.niu.edu/courses/math680/products_withproofs.pdf 